\title{
Cognitive aging, attention, and mild traumatic brain injury
}

Citation for published version (APA):

Klein, M. (1997). Cognitive aging, attention, and mild traumatic brain injury. [Doctoral Thesis, Maastricht University]. NeuroPsych Publishers. https://doi.org/10.26481/dis.19970411mk

Document status and date:

Published: 01/01/1997

DOI:

10.26481/dis.19970411mk

Document Version:

Publisher's PDF, also known as Version of record

\section{Please check the document version of this publication:}

- A submitted manuscript is the version of the article upon submission and before peer-review. There can be important differences between the submitted version and the official published version of record.

People interested in the research are advised to contact the author for the final version of the publication, or visit the DOI to the publisher's website.

- The final author version and the galley proof are versions of the publication after peer review.

- The final published version features the final layout of the paper including the volume, issue and page numbers.

Link to publication

\footnotetext{
General rights rights.

- You may freely distribute the URL identifying the publication in the public portal. please follow below link for the End User Agreement:

www.umlib.nl/taverne-license

Take down policy

If you believe that this document breaches copyright please contact us at:

repository@maastrichtuniversity.nl

providing details and we will investigate your claim.
}

Copyright and moral rights for the publications made accessible in the public portal are retained by the authors and/or other copyright owners and it is a condition of accessing publications that users recognise and abide by the legal requirements associated with these

- Users may download and print one copy of any publication from the public portal for the purpose of private study or research.

- You may not further distribute the material or use it for any profit-making activity or commercial gain

If the publication is distributed under the terms of Article $25 \mathrm{fa}$ of the Dutch Copyright Act, indicated by the "Taverne" license above, 


\section{Cognitive Aging, Attention, and Mild Traumatic Brain Injury}


Neuropsych Publishers Maastrich

M. Klein, Amsterdam 1997

Cognitive Aging, Attention, and Mild Traumatic Brain Injury / Martin Klein. - Maastricht: Neuropsych Publishers Maastricht. - III.

ISBN $\quad 90-75579-05-5$

NUGT. $\quad 744,746$

Neuropsych Publishers is a non-profit organisation which aims at promoting the science of "Brain \& Behaviour" and improving the application of the products of this science in health care and education. Neuropsych Publishers accomplishes these aims by publishing books, dissertations and other products of scientific activity, by disseminating educational materials and publication of tests, assessment scales and other psychometric instruments in the field of Neuropsychology. Neuropsychiatry and other areas within the domain of Brain \& Behaviour.

Postal address:

Neuropsych Publishers

Department of Psychiatry \& Neuropsychology

Section Neuropsychology, Neuropsychiatry, and Psychobiology

Universily Maastrich

PO Box 616

NL-6200 MD Mastricht

The Netherlands 


\section{Cognitive Aging, Attention, and Mild Traumatic Brain Injury}

\section{Proefschrift}

ter verkrijging van de graad van doctor aan de Universiteit Maastricht, op gezag van de Rector Magnificus, Prof. Mr. M. J. Cohen, volgens het besluit van het College van Decanen, in het openbaar te verdedigen op vrijdag 11 april 1997 om 16.00 uur

door

Martin Klein

geboren op 9 februari 1961. te Amersfoort 
Promotor:

Prof. dr. J. Jolles

Beoordelingscommissie: Prof. dr. H.L.G.J. Merckelbach (Voorzitter)

Prof. dr. B.G. Deelman (Rijksuniversiteit Groningen)

Dr. H. Kingma

Prof. dr. Th.W. Mulder (Katholieke Universiteit Nijmegen)

Prof. dr. J. Troost

The studies described in this thesis were carried out at the Mastricht Brain \& Behaviour Institute, the Department of Psychiatry \& Neuropsychology of Maastricht University, and the University Hospital Maastricht, The Netherlands. 
Voor mijn liewe ouders 



\section{Table of Contents}

\section{Introduction}

1.1. Introduction and Justification $\quad 11$

$\begin{array}{ll}1.2 & \text { Aims and Outline of this Thesis } \\ 13\end{array}$

$\begin{array}{ll}1.3 & \text { Questions Addressed in this Thesis } \\ 1.4\end{array}$

1.4 References $\quad 15$

2. Cognitive Aging Theories and Models - An Overview

$\begin{array}{lll}2.1 & \text { Introduction } & 17\end{array}$

2.2 Cognitive Aging Theories and Models $\quad 17$

2.3 Conclusions, and Evaluation of Approaches to Test the Generalized Slowing Hypothesis 21

2.4 References 22

3 Attentional Performance and Cognitive Aging

3.1 Introduction 25

3.2 Attentional Performance and Cognitive Aging 28

3.3 Concluding Remarks 45

3.4 References 46

4 Mild Traumatic Brain Injury, Attention, and Aging

4.1 Introduction $\quad 53$

4.2 Mild Traumatic Brain Injury 55

4.3 Mild Traumatic Brain Injury and Attention 59

4.4 Pathophysiology of Mild Traumatic Brain Injury 68

4.5 Mild Traumatic Brain Injury and Cognitive Aging 69

4.6 A Model for Mild Traumatic Brain Injury as a Risk Factor for
Accelerated Cognitive Aging

$\begin{array}{lll}4.7 & \text { Concluding Remarks } & 81\end{array}$

$\begin{array}{lll}4.8 & \text { References } & 81\end{array}$ 
81 CONTENTS

5 Effect of Test Duration on Age-Related Differences in Stroop Interference

$\begin{array}{lr}\text { Abstract } & 95\end{array}$

5.1 Introduction $\quad 96$

$\begin{array}{lr}5.2 \text { Method } & 97\end{array}$

5.3 Results 99

$\begin{array}{ll}5.4 \text { Discussion } & 102\end{array}$

$\begin{array}{llr}5.5 & \text { References } & 104\end{array}$

6 The Self-Paced Serial Addition Task (SPASAT) and Aging: Predictors of Performance in Divided Attention

$\begin{array}{ll}\text { Abstract } & 107\end{array}$

6.1 Introduction $\quad 108$

6.2 Method 111

$\begin{array}{llr}6.3 & \text { Results } & 115\end{array}$

6.4 Discussion $\quad 119$

$\begin{array}{lll}6.5 & \text { References } & 123\end{array}$

7 The Impact of Aging on Sustained Attention: Time-on-task Effects in Visual Search

Abstract

$\begin{array}{lll}7.1 & \text { Introduction } & 130\end{array}$

$\begin{array}{lll}7.2 & \text { Method } & 131\end{array}$

$\begin{array}{lll}7.3 & \text { Results } & 133\end{array}$

$\begin{array}{ll}7.4 \text { Discussion } & 136\end{array}$

$\begin{array}{llr}7.5 & \text { Refierences } & 138\end{array}$

8 Long-term Persisting Cognitive Sequelae of Traumatic Brain Injury and the Effect of Age

Abstract.

8.1 Introduction

8.2 Method

8.3 Results

8.4 Discussion

8.5 References 
9 Attentional Performance in Young and Old Patients with Cervical Acceleration/Deceleration Injury

$\begin{array}{ll}\text { Abstract } & 155\end{array}$

9.1 Introduction $\quad 156$

$\begin{array}{lll}9.2 & \text { Method } & 158\end{array}$

$\begin{array}{llr}9.3 & \text { Results } & 162\end{array}$

9.4 Discussion $\quad 166$

9.5 References 168

\section{Concluding Remarks}

10.1 Introduction 171

10.2 What is the Nature of the Interaction between Age and Traumatic Brain Injury? 171

$\begin{array}{ll}\text { 10.3 Attentional Functions and Age } & 173\end{array}$

10.4 Similarities and Differences between Traumatic Brain Injury and $\begin{array}{lr}\text { Cervical Acceleration/deceleration Injury } & 1.74\end{array}$

$\begin{array}{lll}10.5 & \text { Mild Traumatic Brain Injury - A Useful Classification? } & 174 \\ 10.6 & \text { Future Directions } & 175\end{array}$

$\begin{array}{lll}10.7 & \text { References } & 176\end{array}$

$\begin{array}{lr}\text { Summary } & 177\end{array}$

$\begin{array}{lr}\text { Samenvatting } & 183\end{array}$

$\begin{array}{lr}\text { Dankwoord } & 189\end{array}$

$\begin{array}{ll}\text { Currifulum Vitae } & 191\end{array}$ 


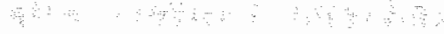

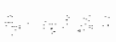




\section{Chapter 1}

\section{Introduction}

\subsection{Introduction and Justification}

According to a recent survey in the United States (Putnam \& Deluca, 1990), clinical neuropsychologists devote a greater proportion of their clinical time to patients who have suffered traumatic brain injury (TBI) than to any other single patient group. When considering only those patients requiring more than 24 hours of hospitalization, the incidence of TBI is comparable to that of Alzheimer's disease (AD) and cerebrovascular accidents (CVAs). The incidence of TBI is 200-300 per 100,000 people in Scotland and the U.S. (Kraus et al., 1984; Miller, 1993), whereas the incidence of mild TBI is 131 per 100,000 people per year (Kraus \& Nourjah, 1989). Unlike AD and CVAs, however, TBI is primarily a condition of the young, with the peak incidence occuring between the ages of 15 and 24 years. For people under the age of 35, TBI accounts for higher rates of death and disability than all other possible causes combined (National Institute of Neurological Disorders and Stroke, 1989).

A reduction in the capacity to process information after mild TBI causes subjects to have lower scores on attentional tasks, as also appears to be the case in cognitive aging. Interestingly, Gronwall (1989) demonstrated that a significant proportion of patients in their twenties with mild TBI showed an attentional performance at 1 week post-injury that was very similar to that of subjects in their eighties. Hartley (1992) states that altered attentional processes are one of the most important contributors to age-related changes in cognition. The changes in cognitive performance associated with normal aging often overlap those accompanying the early, mild stages of AD (Rubin, Morris, Grant, \& Vendegna, 1989). As in normal aging, mernory loss is the most prominent and earliest symptom of AD (Butters, Granholm, Salmon, Grant, \& Wolfe, 1987; Morris \& Kopelman, 1986; Nebes, 1989). However, attentional functions are also affected in the early stages of this disease (Baddeley, Logie, Bressi, Sala, \& Spinnler, 1986; Filoteo et a]., 1992; Nebes \& Brady, 1989; Parasuraman, Greenwood, Haxby, \& Grady, 1992). After reviewing the literature, Parasuraman and Haxby (1993) concluded that visual attention shifting and divided attention were the attentional functions most affected in patients with mild $A D$. They even suggested that attentional changes represent the first cognitive indicator of neocortical dysfunction in early AD. They proposed that a disconnection between frontal and posterior parietal areas underlies the selective disruption of attentional functions in AD. These areas, which might also be affected in TBI, constitute part of the attentional network of the brain (Mesulam, 1990; Posner \& Peterson, 1990). 
Studies investigating the neurocognitive deficits after TBI and in relation to age suggest that persistent effects of TBI sustained earlier in life may remain dormant until the process of physiological aging becomes ewident (Jolles, Houx, Vreeling, \& Verhey, 1993). In fact, previously sustained moderate-to severe head injury has been recognized as a risk factor for $A D$ (Mortimer, 1994), although the potential risk of mild TBI remains elusive. TBI may give rise to diffuse, microscopic damage to the brain, especially the subcortical white matter (Aihara, Hall, Pitts, Fukuda, \& Noble, 1995; Bourke, 1988). Aging is associatted with increased neuronal loss, which, for instance, is expressed by a reduction in brain weight, a reduction in pyramidal neurons, a reduction in dendritic branching (Katzman et al., 1988), and a reduction in cerebral perfusion (Stern, Alexander, Prohovinik, \& Mayeux, 1991). This reduction in functional brain mass represents the reduced brain reserve capacity in the model of Satz (1993), which is an appealing modell to explain why symptoms only become clinically evident later in life. The brain reserve capacity model is based on the assumption that cognitive aging is characterized by a discontinuity caused by some lesion in the central nervous system (CNS). Structural changes become relevant to the individual only when they affect cognitive, motor, or psychosocial functioning. The remarkable plasticity that neural tissue appears to maintain, even into advanced age, and the redundancy of neuronal circuits provide, in most cases, a good deal of functional reserve, even in the presence of age-related structural changes. In the long run, it may be the loss of interconnectivity between neurons, individually and collectively, that eventually compromises the brain's computational power and produces symptoms. Houx and Jolles (1994), Houx, Vreeling, and Jolles (1991), and Jolles and coworkers (1993) suggested that health-related factors possibly associated with brain functioning, so-called Biological Life Events (BLE), may be candidate risk factors for less-than-optimal cognitive aging. TBI is one example of such a BLE; other candidates are chronic medication and alcohol use, and surgical interventions under general anesthesia. Most individuals encounter one or more of these BLE during their lives, but the impact of these BLE usually goes unnoticed. It must be stressed here that lesions which are associated with TBI tend to be quite distinct from lesions associated with aging. In the latter, the term "lesion" refers to the fact that brain tissue is progressively lost with advancing age, whereas TBI is characterized by the sudden onset of damage caused by external factors.

Age at the time of injury has been identified as one of the most important predictors of recovery after TBI (Katz \& Alexander, 1994; Levati, Farina, Vecchi, Rosanda, \& Marrubini, 1982; Naryan, Greenberg, Miller, \& Enas, 1981). As adults get older, morbidity increases and chances for a good recovery decrease. The effect of age on recovery appears to become more pronounced around 40 years according to $\mathrm{Katz}$ and Alexander (1994), who furthermore found that the duration of coma was independent of age, whereas the duration of post-traumatic amnesia increased with age. Long-term recovery in particular is affected by age. Katz and Alexander (1994), for instance, found that among mild TBI patients older than 60 years, $30 \%$ had Glasgow Outcome Scale scores (Jennett, Teasdale, Murray, \& Murray, 1992) that were indicative of rather severe sequelae 12 months after the injury. None of the patients younger than 60 years were severely affected, however. Similarly, when patients with moderate to severe TBI were examined, $54.5 \%$ of the older patients showed severe sequelae at 12 months, whereas only $7.4 \%$ of the patients younger than 20 did.

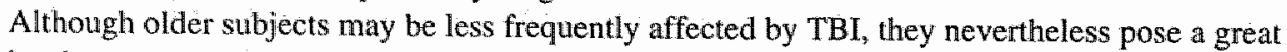
burden on society, since they require more care in terms of length of hospitalization and in terms of support for persisting cognitive deficits (Kraus \& Nourjah, 1989). The rapid increase 
in the number of people living into their 70 s, 80 s and beyond has been one of the most important changes in the developed counties over the last 50 years. Today, the average life expectancy in The Netherlands is about 77 years compared to 43 years in 1900 (Central Bureau voor de Statistiek, 1990). This increased longevity is allso seen in people who sustained TBI at some point earlier in their lives. Because of the increase in life expectancy, we can expect an increase in the duration of disability and dependence associated with TBI, and thus an increased consumption of healthcare resources.

\section{2}

\section{Aims and Outline of this Thesis}

This thesis discusses the plausibility of the thypothesis that even mild TBI may negatively affect the process of cognitive aging. The experiments described this thesis address the hypothesis that there are similarities between the neurocognitive sequelae seen after mild TBI and those seen during aging. The fact that a subject sustained TBI earlier in life, might make him or her more vulnerable to pathological cognitive aging, such as is the case in AD. It is hypothesized that brain injury and aging compromise the brain's reserve capacity. Because of the progressive loss of brain tissue with advancing age, older people already have a reduced information processing capacity. This reduction in capacity is consequently further aggravated by braim injury. After injury older patients usually recover more slowly and sometimes incompletely. Patients with multiple TBI show a similar pattern, which also has been attributed to the effects of neuronal loss. Interestingly, multiple injuries have been recognized as one of the major risk factors for AD later in life. There is ample evidence that several aspects of attention decline with aging and with mild TBI. It is not clear, however, whether the combination of physiological aging and mild TBI leads to accelerated cognitive aging, although the results of studies of severe TBI suggest that this may be the case. The resemblance between the type, but not the extent, of the neuropathological abnormalities in mild, moderate, and severe TBL supports this notion. It is also possible that physiological aging and mild TBI are additive, -instead of increasing the speed at which the subject ages, they rather make the person a few years older. A person who sustained a mild TBI at age 40 , for instance, may show a cognitive performance similar to that of a subject of 60 years old. Finally, it is possible that the effects of mild TBI disappear with the passage of time.

Experimental studies were performed in order to differentiate between these possibilities. Special emphasis is placed on the effects of TBI and age on attentional functions, since these functions appear to be especially affected by cognitive aging and also appear to be compromised after TBI. Impaiments in the ability to efficiently process information from the environment can be divided into four types (Sohlberg \& Mateer, 1989): (a) difficulty in maintaining attentional focus on a single stimulus in the presence of distractors (selective attention), (b) difficulty in dividing attention among two or more stimuli (divided attention), (c) difficulty in switching between subsequent stimuli (switching attention), and (d) difficulty in remaining alert for extended periods of time (sustained attention). The thesis starts with a discussion of the theoretical foundations and models which are used in the experimental chapters. Arguments in favor of a possible additive or interaction effect of age and TBI are summarized, as are relevant findings with regard to the attentional processes as a function of age. The experimental chapters describe the normal course of attentional decline associated with aging and focus on the question whether the process of normal cognitive aging is negatively affected by TBI. 


\section{CHAPTER I}

\subsection{Questions Addressed in this Thesis}

1. Which models are currenty used to explain age-associated cognitive decline?

since most recent studies on human cognitive aging have used speed and accuracy as principal outcome measures, chapter 2 discusses the several explanations for this age-related slowing of test performanice. This chapter concludes with a discussion of approaches to test the generalized slowing hypothesis.

\section{How do attentional functions usually change with age?}

Chapter 3 provides a short description of the history of attentional research and discusses the effect of age on the various attentional domains. Factors that particularly seem to affect attentional performance in the elderly -task complexity and experience-are considered. The possible underlying anatomical mechanisms or circuits of attentional disorders are clarified at the end of the chapter.

\section{Is there evidence that mild TBI accelerates the decline in attemitional performance?}

The cognitive and emotional sequelae of TBI are discussed with particular reference to the attentional domains that appear to be inwolved in TBI and aging. The pathophysiology of mild TBI is discussed in onder to evaluate whether mild TBI can be considered to be a risk factor for accelerated cognitive aging. A model that explains why mild TBI might be a risk factor is discussed at the end of the chapter.

\section{How does selective attention change with age?}

The age-related decline in speed on the Stroop Color Word Test in 429 healthy subjects, aged 25 to 80 years, is discussed in chapter 5 . Other factors that influence selective attention performance, like time-on-task effects, intelligence, and sex, are evaluated.

\section{How does divided atrention change with age?}

Predictors of performance on a self-paced auditory divided attention task were evaluated in a population of 196 healthy subjects aged 25 to 80 years. Chapter 6 also describes the effects of repeated testing with this task, which appears to be particullarly sensitive to cognitive changes after mild TBI. We devised a self-paced task in order to detect whether previously documented deficits in divided attention performance were also also be present when time constraints were not imposed.

\section{How does sustained attention change with age?}

The time-on-task effects in a visual search task administered to 442 healthy subjects aged 25 to 80 years are described in chapter 7 . The relatiwe weights of predictors of performance -initial differences in speed, intelligence, and visual acuity - were assessed. 
7. Is there evidence for accelerated cognitive aging in subjects who howe previously sustaned mild TBI?

Chapter 8 describes the neurocognitive performance of 25 middle-aged and 20 old subjects who had sustained mild-to-moderate TBI several decades earlier. The TBI subjects regarded themselves as normal and healthy. Basic neuropsychological functions were assessed, as was the relation between objectively and subjectively reported cognitive sequelae.

\section{Is there evidence for an accelerated cognitive aging in symptomotic subjects arrenty being} treated for mild TBI?

Chapter 9 describes the influence of cervical acceleration/deceleration injury (CAD) on information processing speed and attentional performance associated with aging. Twenty-nine young ( $<50$ years) and 20 old symptomatic CAD patients ( 250 years) were compared to 49 healthy controls who were matched with respect to age sex, and education. Performance was evaluated with and without time pressure. Time-on-task effects were measured to evaluate possible effects of fatigue.

The tenth chapter concludes this thesis with a general discussion of the findings and with concluding remarks that mainly focus on the application of the scientific results to clinical practice.

\subsection{References}

Aihara, N., Hall, J. J., Pitts, L. H., Fukuda, K., \& Noble, L. J. (1995). Altered immunoexpression of microglia and macrophages after mild head injury. Joumal of Nenrotrawma, $12(1), 53-63$.

Baddelley, A., Logie, R., Bressi, S., Sala, S. D., \& Spinnler, H. (1986). Dementia and working memory. Quarterly Joumal of Experimental Psychology: Human Experimental Psychology, 38(4), 603-618.

Bourke, R. S. (1988). Head Mnjury. Bethesdla, MD NINCDS.

Butters, N., Granholm, E., Salmon, D. P. Grant, I. \& Wolfe, J. (1987). Episodie and semantic memory: A comparison of amnesic and demented pationts. Jourmal of Clinical and Experintental Nearopsychology, 9(5), 479-497.

Centraal Bureau voor de Statistiek (1990). Bevolkingsprognose woor Nederland 1989-2050 (Popwlation progrnosis for The Netherlands 1989-2050). Voorburg. The Netherlands: CBS.

Filoteo, J. V., Delis, D. C., Massman, P. I., Demadura, T., Butters, N., \& Salmon, D. P. (1992). Directed and divided attention in Alzheimer's clisease: Imparment in shifting of attention to global and local stimuli. Journal of Clinical and Experimental Neuropsychology" 14(6),871-883.

Gronwall, D. (1989). Cumulative and persisting effeets of concussion on attention and cognition. In H. S. Lewin, H. M. Eisenberg, \& A. L. Benton (Eds.), Mild Head Injury, (pp. 153-162). New York: Oxford Uniwersily Press.

Harley, A. A. (1992). Altention. In F. I. M. Craik \& T. A. Salthouse (Edls.), Handbook of Aging and Cogrition. (pp. 3-49). Hillsdale, NJ: Lawrence Erlbaum Associates.

Houx, P. J., \& Jolles, J. (1994). Vulnerability factors for age-related cognitive decline. In R. L. Isaacson \& KK. F. Jensen (Eds.). The Vulnerable Brain and Environmental Risks, (pp. 25-41). New York: Plenum Press.

Houx, P. J., Vreeling, F. W. \& Jolles, J. (1991). Rigorous health screening reduces age effect on memory scanning task. Brain and Cognition, 15(2), 246.260.

Jernet, B., Teasdale, G., Murray, G., \& Murray, L. (1992). Head injury. In R. W. Ewans, D. S. Baskin. \& F. M. Yatsu (Eds.), Prognosis of Neurological Disorders, (pp. 85-96). New York: Oxford University Press.

Jolles, J., Houx, P. J., Vreeling, F. W., \& Verhey, F. R. J. (1993). Cognifive aging, biological life events and primitive reflexes. Nenroscience Research Communicalions, 13(Suppl. 1), 47.50. 
Katz, D.I. Alexander, M. P. (1994). Ttaumatic bain injury; Predicting consse of necovery and outcome for patiens admited to rehabilitation. Archives of Nebrology, $5 /(7), 661-670$.

Kutaman, R., Terry, R, DeTeresa, R., Brown, T, Davies, P., Fuld, P., Renbing, X., Peck, A. (1988) Clinica, pathologica, and nearochenical changes in dementia: A subgroup with preserved mental status and

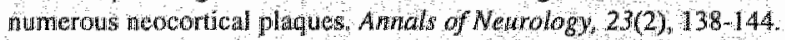

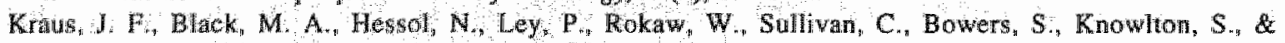
Marshall, L. (1984). The incidence of acute bran injury and serious impament in a defined population. American Joumat of Epidemiology $19(20), 186-201$.

Katus, 1 F. \& Nowrilh, P. (1989). The eprdemiology of mild head injury. In H. S. Levin, H. M. Eisenberg, \& A. L. Benton (Eds. Mild Head Injury, (pp. 8-22). New York: Oxford University Press.

Levat, A., Farina, M.L., Veceh, G.,Rosanda, M. Marubini, M. B. (1982). Prognosis of severe head injuries. Journal of Newrosurgery, 57(6), 779-783.

Mesulam, M. M. (1990). Large-scale neurocognitive netwarks and distributed processing for attention, languge, and menory. Annals of Newrology, 286, 597.613

Miller, J. D. (1993). Head injury Jownal of Nenrology, Neurosurgery and Psychiary, 56(5), 440-447.

Morris, R. G. Kopelman, M, D (986). The menory deficis in Alzhemeratype dementia: A review. Quarterly loumal of Experimenal Psychology: Human Experimental Psychology $38(4)_{1}, 575-602$.

Mortmer, I. A (1994), What are the risk factors for dementia? In $F^{*}$. A. Huppert, C. Brayne, \& D. W. oconnor (Eds), Dementa and Nomal Aging, (pp. 208-243). New York: Cambridge University Press.

Naryan, R. K. Greenberg, R. P., Miller, J. D., \& Enas, G. G. (1981). Improved confidence of oulcome in severe head injury. Jownal of Newroswgery, $54(6), 751-762$.

National Institute of Neurological Disorters and Stroke (1989). Interagency Head-Injury Task Force Repont. Bethesda, MD: National lnstitutes of Health.

Nebes, R. D. (1989). Sentantic memory in Aldhemers disease. Psychological Bulletin, 106(3), 377-394

Nebes, R. D. \& Brady, C. B. (1989). Focused and divided attention in Alzhelner's disease. Cortex, 25(2), 305. 315 .

Parastraman, R., Greenwood, P. M., Haxby, L. V., \& Grady, C. L. (1992). Visuospatial attention in dementia of the Alzheimer type. Brain, 115(3), 711-733.

Parasuraman, R., \& Haxby, J. V. (1993). Altention and brain function in Alzheimer"s disease: A review. Neuropsychology, $7(3), 242-272$.

Posner, M. I., \& Peterson, S. E. (1990). The attention system of the human brain. Annual Review of Newrosicience, 13,2542 .

Putnam, S. H., \& Deluca, J. W. (1990). The TCN professional practice survey: 1. General practices of neuropsychologists in primary employment and private practice settings. Chimical Neuropsychologist, 4(3), $199-243$.

Rubin, E. H., Morris, J. C., Grant, E. A., Vendegna, "T (1989). Very mild senile dementia of the Alatheumer Uype: Clinical assessment. Archives of Neurology, 46(4), 379-382.

Satz, P. (1.993). Brain reservo capacity an symptom onset after brain injury: A formulation and review of evidence for threshold theory. Newropsychology, 7(3), 273-295.

Sohlberg, M. M., Matetr, C. A. (1989). Introduchon to Cognine Rehabilitation. New York: Guilford Press.

Stem, Y., Alexander, G., Prohovnik, I., \& Mayeux, R. (1991). Education provides a cognitive reserve against the alinion manifestations of Alythemen's disease: Evidence from regional cerebral blood fiow. Society for Neruroscience Abstracts, 17.1258 


\section{Chapter 2}

\section{Cognitive Aging Theories and Models - An Overview}

The generalized slowing hypothesis is one of the major hypotheses advanced to explain the cognitive changes accompanying the aging process. In many models and theories, cognitive changes are implicitly related to changes in brain function (Jolles, Verhey, Riedel, \& Houx, 1995). The brain circuitry underlying cognitive performance is formed of anatomical connections between brain areas or between neurons within an area (Posner, 1992; Posner \& Peterson, 1990). Attention organizes the circuitry between brain areas involved in cognitive processing and thus also controls the order in which cognitive operations are performed. The speed at which these operations are performed has been measured in most studies on aging and attention. In fact, speed and accuracy are the only two measures reflecting cognilive performance that have been used in the past decades. Therefore, we need to understand the possible mechanisms underlying the decline in cognitive speed associated with aging. Theories and models of cognitive aging are described as an introduction to the experiments on attentional processes and aging described in this thesis.

Current aging theories and models do not uniquely apply to age-related attentional processes, although some theories or models seem to be especially suited to provide relevant information about which factors may contribute to a decreased attentional performance in the dderly. For further information, the interested reader is referred to the excellent book of Cerella, Rybash, Hoyer, and Commons (1993), which emphasizes the preservation of cognitive functions into old age.

\subsection{Cognitive Aging Theories and Models}

\subsubsection{The Generalized Slowing Hypothesis}

The perhaps most widely used explanation of cognitive aging is the so-called Generalized Slowing Hypothesis proposed by Birren, Woods, and Williams (1980), which is based on the work of Birren (1965). According to Cerella (1985), each component process slows down with 
age, and the slowing of performance of a complex task can thus be predicted from the slowing of performance on a simple task. The generalized decline in fundamental neural events affects all cognitive processes to the same extent (Birren, 1974; Birren ett al., 1980). A task-specific wariant of this hypothesis is that each task component slows at a specific rate, which may or may not be influenced by the rate of slowing of other processes (Cerella, Poon, \& Fozard, 1981). Thus, there could be slowing of the sensorimotor system that is unrelated to slowing of cognitive processes, slowing in the peripheral response system unrelated to central slowing, or slowing of input processes unrelated to output processes. Unfortunately, this hypothesis is too vague to evaluate empirically. Moreower, an ever-increasing amount of evidence suggesting that age has different effects on the cognitive functions subserving speeded information processing (e.g., Cerella, 1990; Salthouse, 1985; for an overview see Bashore, 1993) has made this hypothesis untenable.

\section{2 .2}

The Resource Reduction Hypothesis

Cognitive aging according to this model is the result of an age-related decrease in the availability of fundamental processing resources. This theory states that each stage of information processing requires a specific resource or "fuel" for this task to be performed successfully. Like the Generalized Slowing Hypothesis, the Resource Reduction Hypothesis suggests that the decline un the availability of processing resources has an undifferentiated, quantitative effect on a wide variety of cognitive tasks. This hypothesis has been used to explain age-related differences in attentional performance (McDowd \& Craik, 1988; Plude \& Hoyer, 1986), information processing rate (Salthouse, 1988a; Salthouse \& Kail, 1983; Stine, Wingfield, \& Poon, 1986), working memory (Hartley, 1986), and episodic memory (Jennings, Nebes, \& Yovetich, 1990). Although this hypothesis might be intuitively appealing, the proportion of variance in cognitive aging research explained by a reduced availability of processing resources. is at best $24-30 \%$ (Salthouse, 1988a). Recent electrophysiological studies have provided evidence for multiple attentional mechanisms in the focusing of spatial attention (Luck, 1995). Other studies also seriously challenge the generalizability of this modell (Pashler, 1994; Perfect \& Rabbiu, 1993). As stated by Wickens (1980), the existence of several processing resources underlying attentional performance would reduce the explanatory value of the model. This would be particularly true when the number of processing resounces approaches the number of cognitive tasks. Finally, processing resources are difficult to assess, since specific resources have never been identified precisely or measured independently at either the behavioral or physiological level.

\section{2 .3}

The Disinhibition Hypothesis.

The Disinhibition Hypothesis was formulated on the basis of the results of several studies. The hypothesis considers an age-related decrease in inhibitory capacity as the source of attentiona]. decline in the elderly (e.g., Hamm \& Hasher, 1992; Hasher, Stoltzfus, Zacks, \& Rypma, 1991; Kane, Hasher, Stoltzfus, Zacks, \& Connelly, 1994; McDowd \& Filion, 1992). Tipper (1991) concluded that distracting information interfered with attentional selectivity more in older subjects, and older subjects showed less evidence for inhibitory mechanisms than did younger subjects. The reduction in inhibitory capacity can, for instance, readily be demonstrated by using the Stroop Color-Word Test (Stroop, 1935). According to Hasher and Zacks (1988), 
there is an age-related breakdown in inhibitory mechanisms that control the content of working memory, thus influencing a wide array of cognitive functions. Dempster (1992) stated that resistance to interference (i.e, the capacity for inhibition) is a major factor in the development of intellectual competence and dechining mental ability later in life. The notion of a general decline in inhibitory mechanisms with increasing age, analogous to gameral decline in information processing capacity (Salthouse; 1988b), was rejected some years later by Kramer; Humphrey, Larish, Logan, and Strayer (1994), who concluded that a decrease in the efficiency of inhibitory processes with increasing age is not a general phenomenon, and is not applicable to negative priming effects, response compatibility effects, spatial precuing effects, and self-reported cognitive failures. Kotary and Hoyer (1995) also found no evidence for an age-related decline in inhibitory processes in visual selective attention when they examined distractor interference on a visual search task.

\subsubsection{Perceptual Noise}

The kind of deficit in information processing described by Hasher and Zacks (1988) should be distinguished from the perceptual noise interpretation of Allen, Madden, Groth, and Crozier (1992) and Welford (1984). The perceptual noise hypothesis assumes that there is a filtering deficit whereby older adults have greater levels of internal noise than do young subjects (Allen \& Emerson, 1991; Allen, Groth, Weber, \& Madden, 1993; Allen \& Madden, 1990; Allen, Weber, \& May, 1993). When, for instance, distractor stimuli are visually similar to target items distractor stimuli are misidentified as targets more easily by older adulis than by younger adults. Duncan and Humphreys (1989), moneover, suggested that, for all search materials, difficulty increases with increased similarity between targets and non-targets and decreased similarity between non-targets, producing a continum of search efficiency. They suggested that a parallel stage of perceptual grouping and description is followed by a competitive interaction between inputs, which guides selective access to awareness and action. Allen, Weber, and Madden (1994) concluded that age differences in target-plus-noise tasks must be attributed to differences in the level of activation, and are not likely to be caused by an age-related decrement in retinal capacity.

\subsubsection{The Information Loss Model}

Several mathematical and computational modeling techniques gave rise to models that describe the process of cognitive aging in a quantitative way. The Information Loss Model (Myerson, Hale, Wagstaf, Poon, \& Smith, 1990) is based on the following assumptions: Information is lost during processing, processing occurs in discrete steps with the step duration being inversely related to the amount of information currently available, and aging causes an increase. in the information that is lost at each step. The cognitive process itself, however, remains unafected. These assumptions led the authors to conclude that cognitive aging represents a progressive slowing of successive steps and is manifested by a non-linear relationship between the response speed of young and old adults. Strong support for the hypothesis that cognitiwe slowing is global, and not restricted to specific age-sensitive components, is provided by the fact that the model accurately predicts the latencies of older adults on the basiss of those of 
younger adults, irrespective of the nature of the task (Myerson, Wagstaff, \& Hale, 1994). In a series of silmulations, however, where global versus local information loss and age-related changes in processing speed were compared, Molenaar and Van der Molen (1994) found outcones to be consistently negative: the Information Loss Model falled to discriminate between glotbal and local age effects on the reaction process. As seems to be the case with the previous models, this model is also not specific about which stages of information processing are particularly susceptible to the effects of age.

\section{2 .6}

The Disconnection Model

This model (Cerella, 1990), considers age-related cognitive slowing to be a result of a decrease in information transfer across nodes within the network needed for cognitive operations. When neuronal connections are lost with age, information has to be processed along other, alternative nodes or paths, which conseguently decreases the response speed. Cerella (1990) identified six different patterns of cognitive aging, each characterized by a distinct mathematical equation. He, however, did not specify precisely what task demands were related to the six types of cognitive aging. Another probably more important argument against this model is the fact that aging does not seem to be invariably associated with a decrease in synaptic connections. Swatab (1991), and Swaab, Hofman, Lucassen, Salehi, and Uylings (1994), for instance, suggested that complex experiences in old age can lead to the generation of new synapses in the cerebral cortex and cerebellum. In organs other than the brain, cell activity seems to increase "wear and tear". increasing the rate of aging. However, nerve cell activity seems to lead to the maintenance of neurons during aging and in $\mathrm{AD}$, possibly by preferentially stimulating the action of protective mechanisms such as DNA repair. This "use it or lose it" principle might explain why certain neurons degenerate in aging and $\mathrm{AD}$ while others do not, and why the malfunction of various neuronal systems seen during aging can be reversed by restoring the missing stimulus.

\section{2 .7}

Computer Simulations

The parallel distributed processing theories presented by Cohen, Dunbar, and McClelland (1990), Cohen, Servan, and McClelland (1992), and Rumelhart and McClelland (1986) gave rise to the development of computer simulations. These simulations, for instance, can be used to confirm findings of human psychophysical experiments, such as modeling of the wisual discrimination of three-dimensional objects (Edelman, 1995). According to Salthouse (1988b), information is propagated through five layers of interconnected nodes: an input layer, three hidden layers, and an output or target layer. At each successive step through these layers activation is affected in a negative way. On each pass through the system $10 \%$ of the current activation level is lost. At a certain point, $50 \%$ of the activation is distributed across all nodes connected to the node, whereas the remaining $50 \%$ becomes the new activation level of that particular node, Cognitive aging is simulated by varying the interval between successive waves of propagation through the system. The Salthouse model (Salthouse, 1988b) seems to fit with general findings in cognitive aging research, such as the decrease in processing efficiency with increasing age and the negative effect of task complexity on performance (Cerella, Poon, \& Williams, 1980). 


\section{Conclusions, and Evaluation of Approaches to Test the Generalized Slowing Hypothesis}

One of the strongest arguments in favor of a generalized slowing approach in cognitive aging research stems from the results of the so-called Brinley plot methodology (Brinley, 1965). The Brinley approach predicts the mean cognitive performance of old subjects from the mean performance of young individuals. Intercepts are interpreted as being a reflection of penipheral or perceptual processes, whereas the slope represents central, computational processes that are sensitive to changes in task complexity. After the initial presentation by Brinley (1965), this methodology was adopted by many others (for more recent applications, see Cerella \& Hale; 1994; Fisk \& Fisher, 1994; Myerson et al., 1994). A listorical review of the use of Brinley plots in response time studies of age-rellated cognitive slowing is provided by Bashore (1994). The response times of older subjects appear to be a linear function of the response times of young subjects, supporting the hypothesis of a generalized slowing across tasks.

Questions have been raised about the use of Brinley plots to model generalized slowing, usually in the context of an argument in favor of task-specific slowing. Fisk, Fisher, and Rogers (1992), for instance, demonstrated that Brinley plots were insensitive for discriminating between a pattern of means that indicated the general slowing model and a pattem of means that indicated a task-specific slowing madel. Sliwinski, Buschke, Kuslansky, Senior, and Scarisbrick (1994) pointed at a significant weakness of Brinley plots, namely the dependence on the mean scores, whereas cognitive aging researchers basically are interested in the question whether the speed of performance of any one adult slows across tasks. They furthermore showed that the slowing of performance of older adults depended on the subcomponents of a number adding task. Mayr and Kliegl (1993) concluded that information processing is not a static linear process, but is rather a dynamic process involving working memory. General slowing of mental processes is mediated by higher-order components, such as working memory, and may be evidenced as differences in performance in various tasks. The use of time-accuracy functions for two pairs of tasks that differ in cognitive complexity - word scanning versus cued recognition, and figural scanning versus figural reasoning - led Kliegl, Mayr, and Krampe (1994) to conclude that different slowing factors are required for high- and low-complexity tasks. The cognitive aging hypothesis, in which a single slowing factor can account for interactions between age and cognitive task complexity thus seems to be untenable. Cross-sectional and longitudinal response time data showed slowing of simple response time and relatively greater slowing of dysjunctive response time across decades. Repeated testing of the same subjects over 8 years showed consistent slowing and increased variability with age. These findings are consistent with the hypothesis that slowing of behavior is a direct function of task complexity (Fozard, Vercryssen, Reynolds, Hancock, \& Quilter, 1994).

Finally, current knowledge of the neural substrate subserving cognitive performance also does not support the idea of a generalized decline in cognitive cognitive performance with increasing age. The distinct highly specialized brain regions involved in information processing (e.g., Bondareff, 1985; Posner \& Dehaene, 1994; Posner, Petersen, Fox, \& Raichle, 1988; Zeki, 1991) have distinct phylogenetic histories and developmental trajectories, and do not age uniformly (Bondareff, 1985; Marr, 1982). This finding urges cognitive aging researchers to develop new methodologies to detect which cognitive processes might or might not be affected 
by physiological aging.

Current mainstream cognitive and developmental research is characterized by a so-called Cognitive Neuroscience Approach (Johnson \& Rybash, 1993), which aims at integrating behaviorat, psychophysiological, and neuroanatomical approaches in the study of attention (e.g, Kinchla, 1992, Posner, 1992; Posner \& Peterson, 1990). Modern technological breakthroughs, such as PET, ERPs, and high resolution Magnetic Resonance Imaging (MRI; for review, see Posner \& Carr, 1992), enable cognitive aging researchers to identify the unique demands of specific stages of information processing and the brain areas involved in these mental operations. The use of the cognitive neuroscience methods allows performance to be broken down into a group of component operations, where especially the combined studies usingsuch that combination of anatomical methods (PET) and methods sensitive to time-dynamic change (ERPs) provide a convenient way to trace rapid time-dynamic ehanges that occur in the course of human information processing (Posner \& Raichle, 1994). The second hald of the "Decade of the Brain" may provide us with new information about the possible relewance of the cognitive neuroscience approach to solving scientific problems in cognitive aging research.

\section{4}

\section{References}

Allen, P. A, \& Enerson, P. L. (1991). Holism revisited: Evidence for parallel independent word level and letterlevel processors during word recognition. Joumal of Experimental Psychology: Human Perception and Perfomianck $17(2), 489-511$.

Allen, P. A., Croth, K. E., Weber, "T. A., \& Maddan, D. J. (1993). Influence of response selection and noise similarty on age diflerences in the redundancy gann. Joumats of Gerontology: Psychological Sciences, 48(4), $189-198$.

AHen, P. A., \& Madden, D. J. (1990). Evidence for a parallel input serial analysis molel of word processing. Jownat of Experiwental Pychology: Howan Perception and Performance, 16(1), 48-64.

Allen, P. A., Madden, D. J., Groth, K. E., \& Crozier, L. C. (1992). Impact of age, redundancy, and perceptual noise on visual search. Jowrials of Gerontology: Psychological Sciences, 47(2), 69-74.

Allen, P. A., Weber, T. A. \& Madden, D. J. (1994). Adult age differences in attention: Filtering or selection? Joumalis of Cerontorlogy: Psychological Sciences, 49(5), 213-222.

Allen, P. A., Weber, T. A. \& May. N. (1993). Age differences in letter and color matching: Selective attention or interinal noise Journats of Gerontology: Psychological Sciences, $48(2)_{4} 69.77$.

Bashore, T. F. (1993). Differental affects of aging on the neurocognitive functions subserving speeded mental processing. In J. Cenchla, J. Rybash, W. Hoyer, \& M. L. Commons (Eds.), Adult Information Processing: Limits on Losi, (pp. 37-76). San Diego: Academic Press.

Bathore, E. R. (1994). Some thoughts on neurocognitive slowing. Special Issue: Life span changes in human performance. Acta Psychologica. $86(2-3), 295-325$

Birien, J. (1974). Psychophysiology and the speed of response. American Psychologist, 29, 808-815.

Birren, J., Woods, A. M. \& Willams, M. V. (1980). Behavioral slowing with age: Causes, organization and consequences. In L. W. Poon (Ed.), Aging in the 1980s, (pp. 293-308). Washington, DC: American Psychological Associntion.

Bimen, J. E. (1965). Age changes in speed of behavior: Its central nature and physiological correlates In A. T. Welford \& I. E. Bitren (Eds.), Behavior, Aging and the Nenous System, (pp.191-216). Springfield, IL: Charles C. Thomas.

Bondareff, W. (1985). The neural basis of aging. In J. E. Birten \&. K. W. Schaie (Eds), Hortbook of the Psychology of Aging, (2nd ed. pp. 95.112). New York: Van Nostrand Reinhold.

Brinley, J. F. (1965). Cognitive sets, speed and accuracy af performance in the elderly. In A. T. Welford \&. J. E. Bimen (Eds.), Bethawion, Aging and the Nenous System. (pp. 14-149). Springfield, IL: Charles C. Thomas.

Cermila, J. (1985), Information processing rates in the elderly. $P_{5 y c h o l o g i c a l}$ Bulletin, $98(1), 67-83$. 


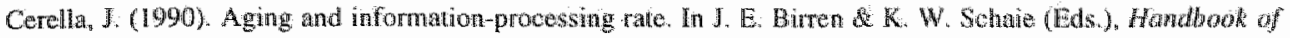
the Prychology of Aging. (3nd ed. pp, 201-221). New York: Academe Press.

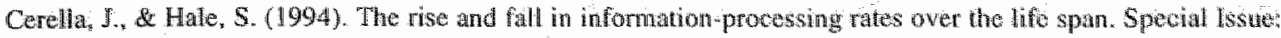
Life span changes in human pertomance. Acto Psychologica, $8602-3), 109-197$.

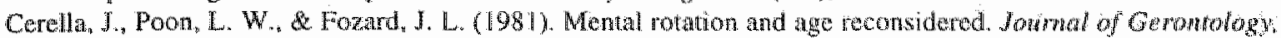
$36(5) .620-624$.

Cerella, J., Poon, L. W., \& Willams, M. V. (1980). Age and the complexity hypothesis. In L. W. Poon (Bd.) Aging in the 1980, (pp. 332,340). Washington, DC: Ancrican Psychologitol Association.

Cerella, J., Rybash, J., Hoyer, W., \& Commons, M. L. (1993). Adult Information Processing: Limirs on Lass. San Diego: Academo Press.

Cohen, I. D., Dunbar, K. \& McClelland, J. L. (1990). On the control of antomatic processes: A paralle Jistributed processing account of the Stroop effect. Prychological Rewew, 97(3), 332-361.

Cohen. J. D., Sevar, S. D. McClelland, I. L. (1992). A paralle distributed processing approach to automaticity. Special Issue: Views and varieties of atumaticity. American Jownal of Psychology, 105(2); 239-269.

Dempster, F. N. (1992). The rise and fall of the inhibitory mechanism: Toward anified ineory of cognitive developmen and aiging. Developmenot Review, $12(1), 45-75$.

Duncan, J. Humphreys, G. W. (1989). Visual search and stimulus simiarity. Paychologicat Review, 96(3), $433 \times 458$.

Edelman, S. (1995). Representation of similarity im three-dimensional object discrimination. Netral Computation, $7(2), 408-423$

Fisk, A. D., \& Fisher, D. L. (1994). Brinley plots and theories of aging: The explicit, muddled, and implicit debates. Jownals of Gerontology: Psychological Sciences, 49(2), 81-89.

Fusk, A. D., Fisher, D. L., \& Rogers, W. A. (1992). Getheral slowing alone cannot explain age-telated search effects: Reply to Cerella 1991. Journal of Experimenus. Pychology: General, 121(1).73-78.

Fozard, J. L., Vererysen, M, Reynolds, S. L., Hancock. P. A., \& Quilter, R. E. (1994). Age differences and changes in reaction time: The Baltimore Longitudinal Study of Aging. foumals of Geromology: Psychological Sciences, 49(4), 179-189.

Hamm, V. P., Hasher, $\mathrm{I}$. (1992). Age and the availability of inferences. Psychology and Aging, $7(1), 56-64$.

Hartley, J. T. (1986). Reader and text vamables as deteminants of discourse nemory in afulthood. Psychology and Aging, $1(2), 150-158$.

Hasher, L., Stoltzfus, E. R., Zacks, R. T., \& Rypma, B. (1991). Age and inhibition. Joumal of Experimental Psychology. Leaming. Menony, and Cognition, 17(1), 163-169.

Hasher, L. \& Zacks, R. T. (1988). Working menory, comprehension, and aging; Review and a new view. Iry $\mathrm{G}$ H. Bower (Ed). The Psychology of Leaming and Morvation, (Vol. 22, pp. 193-225). Orlando: Academic Press.

Jennings, J. R, Nebes, R. D., Yovetich, N. A. (1990). Aging increases the energetic demands of episodic memory: A cardiovascular analysts. Jonnal of Experimental Psychology: General, 119(1), 77.91.

Johnson, S. H., \& Rybash, J. M. (1993). A cognitive neuroseience perspective on age-related slowitg: Developmental changes in the funcional architecture. In J. Cerella, J. Rybash, W. Hoyer. M. L. Commons (Eds.), Adult Information Processing. Limits on Lons (pp, 158-173). San Diego: Acadentic Press.

Jolles, J., Verhey, F, R. J., Riedel, W. J., \& Houx, J. J. (1995). Cognilive impainment in the olderly: Predisposing factors and implications for experimental drug studtes. Drugs \& Agimg, 7(6), 459-479.

Kane, M. I., Hasher, L., Stoltufus, E. R., Zacks, R. T., \& Connelly, S. L. (1994). Inhibitory antternional mechanisms and aging, Psychology and Aging, $9(1), 103-112$.

Kinchia, R. A. (1992). Altention. Annual Review of Psychology, 43,711-742.

Kliegl, R., Mayr, U., \& Krampe, R. F. (1994). Timewcenracy functions for determining process and petson

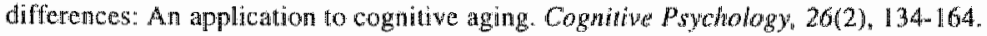

Kotary, L.., \& Hoyer, W. J. (1995). Age and the ability to inhibit distractor information in visual selective attention. Experimenal Aging Research, 2/2) 159 171.

Kramer. A. F., Humphrey, D. G., Larish, J. F. Logan, G. D., \& Strayer, D. L. (1994). Aging and inhibition: Beyond a ninary wiew of inhibitory processing in altention. Psychology and Aging, 9(4), $491-512$.

Luck, S. 1. (1995). Multiple mechanisms of visual spatial attention: Recent evidence from humam electrophysiology. Behawioural Brain Research, $7 /, 1-2$.

Marr, D. (1982). Virion. San Francisco: Freeman.

Mayr, U., Kliegl. R. (1993). Sequential and coordinute complexity. Age-based processing limitations in 
figural tramsiformations, Joumal of Experimental Psychology: Leaming, Memony, and Cognition, 19(6). 1297.1320 .

McDowd J. M. \& Craik, F. I. M. (1988) Effects of aging and task difficully on diwided attention performance. Journal of Experimental Psychology: Human Perception and Performance, 14(2), 267-280.

McDowd. J. M. Filion, D. L. (1992). Aging, selective attention, and imhibitory processes: A psychophysiological approach. Psychology and Agüng, 7(1), 65-71.

Molenaar, P. C. M., \& Van der Molen, M. W. (1994). On the discrimination between glabal and local trend hypotheses of life-span changes in processing speed. Special Issure: Life span changes in human performance. Acta Psychologica, 86(2), 273-293.

Myerson, J., Hale, S., Wagstaf, D., Poon, L. W., \& Smith, G. A. (1990). The information-loss model: A mathenatical theory of age-related cognitive slowing. Psychological Review; 97(4), 475-487.

Myerson, 1. Wagstaff, D. \& Hale, 5. (1.994). Brinley plots, explained variance, and the analysis of age differences in response latencies. Journals of Gerontology: Psychological Sciences, 49(2), 72-80.

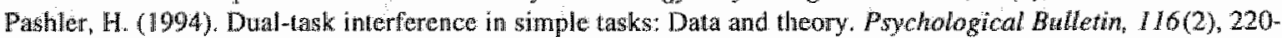
244.

Petfect, T, J., \& Rabbin, P. M. (1993). Age and the divided attention casts of category exemplar generation. British Journal of Developmental Psychology, $/ /(2), 131-142$.

Plude, D. J., \& Hoyer, W. J. (1986). Age and the selectivity of wisual information-processing. Psychology and Aging, I(1), 4-10.

Posner, M. I. (1992). Attention as a cognitive and neural system. Current Directions in Psychological Science. I(1). $11-14$.

Posner, M. I. \& Carr; T. H. (1992). Lexical access and the brain: Anatomical constraints on cognitixe models of word recognition. American Joumal of Psychology. 105(1), 1-26.

Posner, M. I., \& Dehaene, S. E. (1994). Attentional networks. Trend's in Neurasciences, 17(2), 75-79.

Posner, M. I., Petersen, S. E., Fox, P. T., \& Raichle, M. E. (1988). Localization of cognitive operations in the human brain. Science, 240(4859)。 1627-1631.

Posnen, M. I., \& Peterson, S. E. (1990). The attention system of the human brain. Annual Review of Neuroscience, 13,25-42.

Posner, M. 1, \& Raichle, M. E. (1994). Images of Mind. New York: Scientific American Library.

Rumelhart, D. E. d McClelland, J. L. (1986). Parallel Distributed Processing: Explorations in the Microstructure of Cognution. (Vol. 1). Cambridge, MA: Bradford.

Salthouse, T. A. (1985). Speed of behavior and its implications for cognition. In J. E. Birren \& K. W. Schaie (Eds.), Handbook of the Psychology of Aging, (2nd ed. pp. 400-426). New York: Van Nostrand Reinhold.

Salthouse, T, A. (1988a). Initiating the formalization of theories of aging, Psychology and Aging, 3(1), 3-16,

Salthouse, T. A. (1988b). Resource-reduction interpretations of cognitive aging. Developmental Review, 8(3), 238272.

Salthouse, T. A., \& Kail, R. (1983). Memory development throughout the life span: The role of processing rate. In P. B. Battes \& O. G. I. Brim (Eds.), Life-Span Development and Behavior. New York: Academic Press.

Sliwinski, M., Buschke, H., Kuslansky, G., Senior, C., \& Scarisbrick, D. (1994). Proportional slowing and addition speed in old and young adults. Psychology and Aging. 9(1), 72-80.

Stime, E. L. Wingfield, A., \& Poon, L. (1986). How much and how fast: Rapid processing of spoken language in older adul hood. Psychology and Aging, l(4), 303-311.

Stroop, d. R. (1935). Studies of interterence in serial verbal reactions. Journal of Experinental Psychology, 18, $643-662$.

Swanb, D. $\mathbb{F},(1991)$. Brain aging and Alzheimer's disease: "Wear and tear" versus "use it or lose it". Newrobiology of Aging. 12(4), 317-324.

Swab, D. F., Hofmam, M. A., Lucassen, P. J, Salehi, A., \& Uylings, H. B. (1994). Neuronal atrophy, not cely death, is the matin hallmark of Altheimer's disease. Neurobiology of Aging, 15(3), 369-371.

Tipper, S. P. (1991). Leess attentional selectivity as a result of declining inhibition in older adults. Bulletin of the Psychonomic Society, 29(1), 45-47.

Welford, A. T. (1984). Between bodily changes and performance: Some possible reasons for slowing with age. Experimental Aging Research, 10(2), 73-88.

Wickens, C. D. (1980). The structure of attentional resources. In R. Nickerson (Bd.), Attention and Performance, (Vol. VIII, pp. 239-258). Hillsdale, NJ: Lawrence Erlbaum Associates.

Zeki, S. (1991). A thought experiment with positron emission tomography. Ciba Foundation Symposium, 163, $145-154$ 


\section{Chapter 3}

\section{Attentional Performance and Cognitive Aging}

"The remote effects of attention are too incalculable to be recorded. The practical and theoretical life of whole species, as well as of individual beings, results from the selection which the habitual direction of their attention involves."

- William James -

\section{1}

Introduction

This much-quoted remark of James (1890) on attention fortunately no longer reflects the current state of attentional research. With regard to the effects of aging on attention, however, this statement may still be true. Apart from methodological considerations, the differences in individual trajectories of cognitive aging strongly influence conclusions about the extent to which attentional functions are affected by the process of cognitive aging. In most individuals old age is accompanied by a decrease in the performance of various cognitive domains. Apart from performance on sensitive cognitive tasks, many elderly people complain of diminishing cognitive abilities (for review, Botwinick, 1981; Huppert, Brayne, \& O'Connor, 1994; Jolles, 1986; La Rue, 1992). Aging furthermore appears to affect the ability to integrate and plan new activities, to make decisions, or to solve problems (Reese \& Rodeheaver, 1985). Several authors suggest that attentional capacity is invariably poorer in old individuals than in young individuals (Hartley, 1992; Plude \& Hoyer, 1985). Greenwood and Parasuraman (1994), among others, however, suggest that cognitive aging might not affect all attentionall domains. This and other issues with regard to the complex relation between attention and cognitive aging are the topic of the present chapter.

It is not the primary aim of this chapter to provide an in-depth discussion of attentional research since Broadbent proposed his attention-related information processing models in 1958. The primary focus is on attentional research in the perspective of cognitive aging. Therefore only some milestones of the history of attentional and related research are presented in Table 3.1 and are discussed in the next paragraph. 


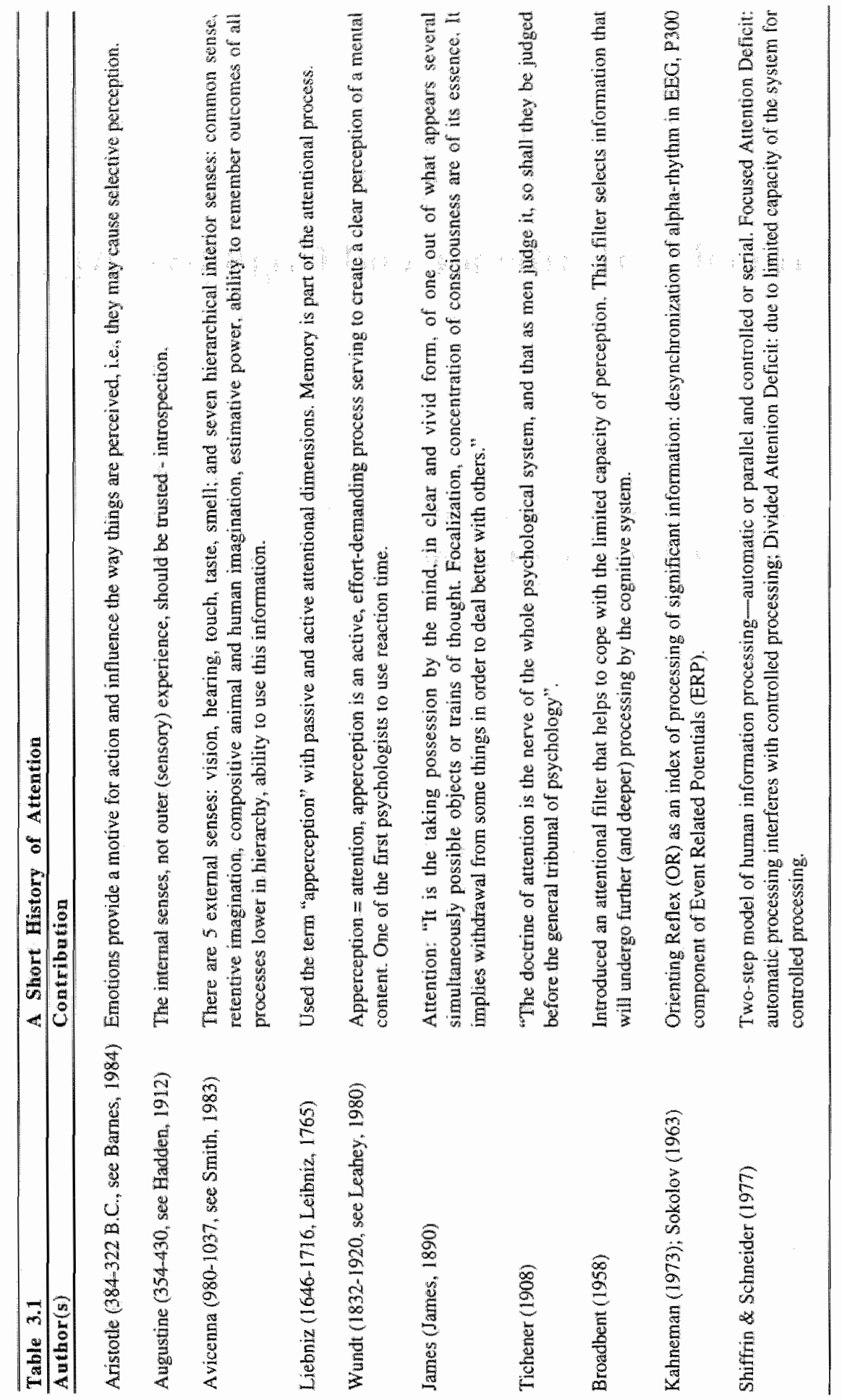




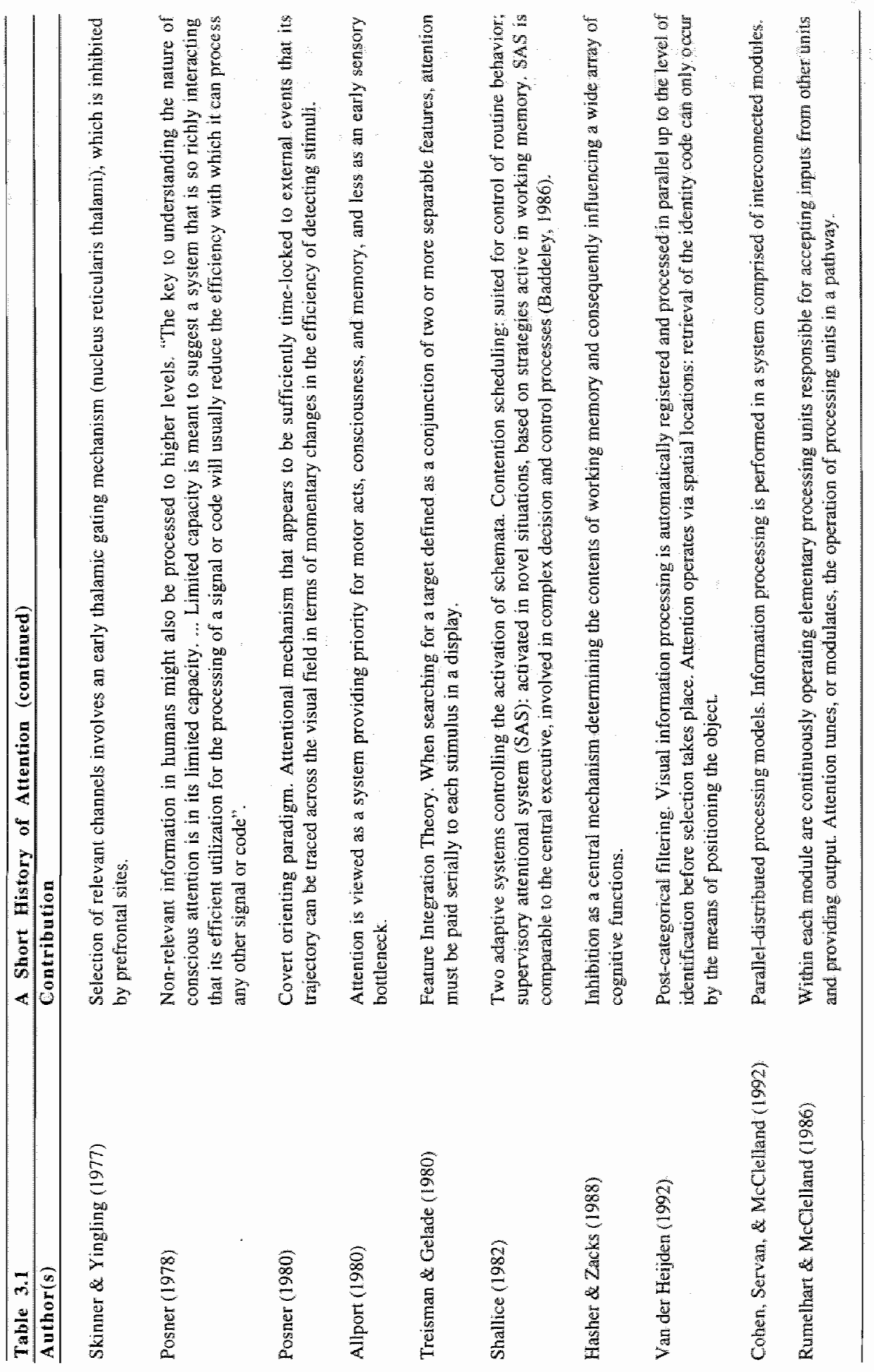


For readers who are interested in the perceptual, selection, and control aspects of attention, the book edited by Baddeley and Weiskrantz (1993) is recommended, which is a tribute to the pioneering work of Broadbent. Readers who are specifically interested in the determinants of selective attention should read the work by Van der Heijden (1992), and if they are interested in inhibitory mechanisms in attention, memory, and language, they should read the work of Dagenbach and Carr (1994). Finally, a more neuropsychological approach is represented by the excellent book of Van Zomeren and Brouwer (1994).

\section{2}

\section{Attentional Performance and Cognitive Aging}

Although most of our understanding of attention is based on studies initiated after World War II (Broadbent, 1958), the concept of attention appear in Greek and Roman texts (400 B.C.). The work of Aristotle (see Barnes, 1984) is one of the first that in some way refers to the rolle of selectivity in the way the outside world is perceived. The first empirical studies of perception and attention-like phenomena probably date back from the eighteenth century, when Liebniz (1765) was working on experiments concerning attention and memory. After the studies of Wundt (see Leahey, 1980), James (1890), and Tichener (1908), interest in attentional research waned for some decades, mainly due to the rise of the behavioristic school, which did not consider attentional processes to be of great value in explaining and predicting human cognitive behavior.

The influential book of Broadbent (1958) brought about a revival in attentional research in the postwar years. Based on theories of information processing, several models have been proposed to explain how the selection of sensory information takes place. "The "filter" theories propound that irrelevant signals are filtered out so that meaningful messages can be processed without difficulty (Broadbent, 1958; Broadbent, 1971). Information processing theories attribute various areas in the CNS, where the main selection of information takes place (Skinner \& Yingling, 1977), a role in information processing. Some theories emphasize the very fine sensory relays as being the most important component in the process of selecting relevant information (Motan \& Desimone, 1985). Other theories suggest that, during the processing of information, different types of associations are established according to a specific hierarchy. In this manner, selection occurs at different levels. There are various schools of thought about the locus, if any, where information is selected. Some investigators are advocates of localizationism, globalism or anti-localizationism, while others suggest the existence of a functional hierarchy or a link between distributed systems (Rumelhart \& MoClelland, 1986). The latter derives from Hebb's concept of neuronal assemblies (Hebb, 1949), which eventually was incorporated in Mountcastle's definition of modules of information processing (Mountcastle, 1986; Mountcastle, Lynch, Georgopoulos, Sakata, \& Acuna, 1975).

Shiffrin and Schneider (1977) presented a two-step model of information processing, in which a distinction was made between automatic, overlearned, or well-trained information processing, and controlled, conscious effort-demanding information processing (see also Fisk \& Scerbo, 1987; Schneider \& Shiffrin, 1977). The duration of controlled processing depends on the number of hypothetical cognitive steps needed for a task. These conscious steps occupy space in working memony, thus leading to a decreased capacity for the performance of concurrent 
tasks (Baddelley, 1986). Salthouse (1994), however, suggests that although increased age might be associated with lower scores on measures of working memory functioning, many of the age-related influences appear to be mediated by a slower speed of information processing. Furthermore, recent studies indicate that slower processing primarity influences the time required to achieve a stable encoding of the information rather than the rate at which information is lost or the rate of subsequent processing of information. Shiffrin and Schneider (1977) described two kinds of attentional problems: focused attention deficits (FADs) and divided attention deficits (DADs). FADs can be demonstrated in spatial precuing wisual search experiments, where the most probable location of a target is defined (Rabbitt, 1982). FADs occur when the subject is unable to ignore distractor information, even though he or she knows. in advance where to expect a target. $A D A D$ occurs when the number of possible target locations increases and thus too much task-relevant information is presented too quickly. Hence, relevant signals will be missed or required responses can not be carried out efficiently. Plude and Doussard Roosevelt (1989) used the Feature Integration Theory (Treisman \& Gelade, 1980) as a means of determining the point during processing at which selective attention deficits arise and states that the contribution of age-related limitations in data handing is secondary to the contribution of age-related decreases in selective attention.

With the advent of techniques that allow better temporal and spatial resolution of brain systems, the study of brain function in general, and of cognitive processes in particular, has attained a new momentum. Using imaging techniques in patients with localized lesions, or in animal models, it has been possible to determine which brain regions participate in attentional processes. Regions with important interconnections with the brain stem, diencephalon, and cerebral cortex appear to form specific neuronal pathways and ensembles involved in attention. More recently, Posner (1995) and Posner and Raichle (1994) suggested that cognitive functions are made up of assemblies of elementary operations occurring at defined levels of the brain. The brain areas most likely to be involved in attentional performance are discussed at the end of this chapter.

Modern neuropsychology views attention not as a unitary function, but rather as an integrative mechanism that serves to transform environmental events into mental representations. Several formal models have been proposed, and some effort has been made to apply these models to clinical populations. Only relatively recently, however, have psychologists begun to examine the change in attention over the adult life span. This lag in initiative in research in older adults is somewhat surprising in the light of the fundamental nature of attention as a prerequisite for almost any cognitive behavior and its importance for effective behavior and survival. A common feature of most current attentional models is that they emphasize the central role of selection and intensity or capacity. Attention is qualified by selection and quantified by the intensity aspect. The more recent theories (Cohen et al., 1992; Hasher \& Zacks, 1988; Van der Heijden, 1992) emphasize the qualitative aspect in particular. The terms attention, effort, capacity, and mental resources used in these studies are used synonymouslly to refer to an underlying limited resource needed for selection and execution of various tasks.

In the next few paragraphs the possible effect of aging on attention is discusssed with reference to the most common classification of attentional task performance - selective attention, divided attention, shifting attention, and sustained attention. These attentional domains are defined in Table 3.2. It should be emphasized, however, that the different aspects of attention might interact at different levels. Target detection in a sustained attention task, for instance, depends heavily on the selective attention capacity of the individual, as is the case for divided attention 
performance. This artificial classification is based on theoretical grounds rather than on empirical findings.

\section{Thable 32 Definitions of Attenthon}

Doman Definition

Selective Altontion:

Focusing on and shifting attention among different stimulus locations, features, or catcgories selection of higher levels of processing, while preventing access of other signals to these high levels of processing (Posner, 1988).

Divided Attention

Performance of two of mone simulaneous tasks. Parallel processing of several inpus (McDowd \& Birren, 1990 ).

Shifting Altention

Alternately monitoring two or more sources of input. Serial processing (MeDowd \& Birren, 1990).

Sustained Attention

Detection of infrequent and unpredictable events, particularly after prolonged periods of observation (Parasuraman, Warn, \& Dember, 1987).

\section{2 .2}

Selective Altention

Selective attention involves focusing on and shifting attention among different stimulus locations, features, or categories. According to Posner (1.988), attention involves the selection of higher levels of processing, including conscious processing, while preventing other signals from accessing the same levels of processing. Selective attention is generally evaluated by tests such as cue-directed attention, visual search and memory search, dichotic listening, and the Stroop Color Word Test (Stroop, 1935).

The two main features particularly involved in selective attention performance are selectivity and capacty. Selectivity enables the subject to distinguish between relevant and irrelevant information, whereas the capacity aspect denotes the resources available for controlling information processing. The selectivity and capacity dimensions of attention are not completely independent, however, but rather work together to enhance attentional performance. Selective mechanisms limit the amount of information (i.e, only relevant information) to be processed. Furthermore, if the information cannot be processed automatically, but demands controlled processing, the selective mechanism ensures that only relevant information enters working memory, which has a limited capacity.

\section{Cuing Experments}

Several studies point to an age-related decrease in performance on tasks that measure selective attention. This decrease in task performance does not appear to be affected by a deterioration in selectivity per se, but can be attributed to a decrease in the capacity aspect of selective attention (Madden, 1990a). Nebes and Madden (1983) found equivalent cuing effects in young and older subjects. A cue presented before presentation of a signal led to a significant reduction in response time that was the same in both young and old subjects. Nebes and Brady (1989) 
replicated this finding and moreover found that the beneficial cuing effect was unaffected even in patients suffering from $\mathrm{AD}$. Older adults may preserve the capacity to focus attention on the spatial location of relevant display items in visual search tasks, as has been demonstrated by Madden $(1983,1986)$. The magnitude of the cuing effect was similar in young and old subjects. Madden (1986) also found that the effect of response-incompatible items in non-cued locations was conistently affected by age. Madden (1992) found that the effect of varying the cue validity favored an interpretation of selective attention in terms of a processing gradient rather than a zoom lens. Although the allocation of attention across trials was similar for young and older adults, there was an age-related increase in the time required to allocate attention within individual trials. McCalley, Bouwhuis, and Juola (1995) concluded that a single flexible resource allocation (ring) model of attention in a spatial cuing task could account for aging effects when stimulus visibility was controlled for.

An age-related decline in response time can be detected when the cue is presented at a central location and possible targets are at the left or right of this cue. Nissen and Corkin (1985), for instance, found older subjects to have a greater increase in response time than young subjects on incorrectly cued trials. This finding, however, was not confirmed by Hartley kieley, and Slabach (1990) and Folk and Hoyer (1992), who found that the magnitude of cuing effects with peripheral cues was at least as great for older adults as for younger adults. Similar results were obtained with symbolic cues, although cuing effects for older adults varied with cue difficulty. Folk and Hoyer (1992) concluded that cue encoding may decline with advancing age but that the efficiency of the shift process is preserved. The time course of cuing effects, however, might be affected by age. When a centrally presented cue was present for less than 750 milliseconds, younger subjects showed significantly stronger response time cuing effects than older subjects (Hoyer \& Familant, 1987). Madden (1990b) presented a cue 50-183 milliseconds prior to display onset and found response time to increase as a function of both the distance of the target from fixation and the presence of non-target letters in the display. Response time effects were more pronounced in older subjects than in young subjects. When non-target letters were present in the display, the build up of attention over the cue-target interval was slower for older subjects than for young subjects. This age difference was independent of the generalized age-related slowing.

\section{Search Tasks}

Recall of stored representations can facilitate identification of items in a visual display as was demonstrated by Christie and Klein (1995). They presented a familiar item (word) and an unfamiliar item (nonword) simultaneously at unpredictable locations, and after varying delays, moved one of the stimuli. At the shortest intervals, subjects were more efficient at discriminating the motion of the familiar item. Madden (1987) found that the semantic properties of the stimulus display in a visual search task influenced the efficiency and attentional demands equally in the groups they tested. Allen, Madden, Groth, and Crozier (1.992) repeatedly presented the same target and found a greater reduction in the interfering effects of non-target items in older subjects than in younger subjects. Fullerton and Smith (1980) displayed strings of eight letters which varied in the degree to which they resembled the orthographic structure of English. Search performance improved when the strings of letters approximated English words; the decrease in response time was comparable for young and old subjects. Apart from replicating this finding, Nebes and Boller (1987) also showed that even patients suffering from $\mathrm{AD}$ were able to use orthographic redundancy in a visual search task. 
Memory-driven selective attention also appears to be characterized by an age constancy. In a typical memory search task, subjects have to respond to the presentation of digits or letters and have to answer positively when the digits or letters are member of a memorized set. When letters are ordered in a highly familiar sequence $(e, g, a, b, c, d, e)$ rather than in a less familiar sequence, older subjects show a greater performance increment than younger subjects (Thomas, Waugh, \& Fozard, 1978). Madden (1985) investigated the ability of young and elderly adults to use memory-driven selective attention in two visual search experiments. In the first experiment a yes-no search paradigm was used with it stimulus-onset-asynchrony ( $\mathrm{SOA}$ ) varying from 200 to 1000 milliseconds. The second experiment was the same as the first, but one of the two target letters was presented as a cue. Both young and old adults exhibited faster RTs to a visual display on trials when a cue correctly predicted the particular target letter that would be present in the display. Variations in the SOA between the cue and the display demonstrated that both age groups could focus attention in expectation of a particular target letter within 200 milliseconds. Results indicate that age differences in performance were determined primarily by quantitative changes in the speed of information processing rather than by qualitative changes in attention. In both experiments, however, the two age groups differed in the type of relationship between speed and accuracy that they adopted, suggesting a possible age difference in performance strategy. Some earlier experiments suggest that there is an age-related decline in memory-driven selective attention. In an experiment by Rabbitt (1964), older subjects appeared to benefit less from correctly cued trials than did younger subjects. On incorrectly cued trials, however, the increase in response time was equivalent both for young and old subjects. These findings were replicated by Rabbitt and Vyas (1980), who furthermore found that only young subjects exhibited a significant decrease in response time when the same display items were repeatedly presented across trials.

\section{Dichotic Listening}

Although the capacity aspect of performance of selective attention tasks appears to be affected by aging in a negative way, the reorientation of attention also appears to be affected. Panek and Rush (1981), for instance, assessed the maintenance and reorientation components of attention by using an auditory dichotic listening task and found a significant decrease in both maintenance and reorientation of attention with increasing age. They also found the reorientation of attention to be more difficult than attention maintenance, particularly for older adults. They suggested that the inability to reorient attention is primarily responsible for the manifest differences in selective attention ability of older adults. Barr and Giambra (1990) found dichotic shadowing error rates to increase with age, even when they attempted to control for the effect of age by adjusting for age differences in monaural shadowing errors, fluid intelligence, and pure-tone hearing loss. These results showed that there are selective attention deficits in older adults that cannot be accounted for by the uncertainty of the target location. Martin and Cranford (1991) administered a dichotic digit recognition task in which subjects were required, in separate sessions, to repeat all digits heard in both ears (channel capacity measure), or to report the digits in one ear while ignoring the digits in the other ear (selective attention measure). With both measures, older subjects performed significantly poorer than younger subjects. Although similar age-related decreases in performance were observed in behavioral and electrophysiologic tests, no significant correlations were found between performance in the two types of tests (Cranford \& Martin, 1991). This suggests that while both behavioral and electrophysiologic tests can reveal age-related decreases in binaural processing, these defects do not necessarily reflect the same 
underlying neural processes. In contrast to Crantord and Martin (1991), Woods (1992) found performance to be little affected by aging in a dichotic selective attention task using Event Related Potential measures. Response time, target detection accuracy, and false alarm rates were similar in young and old subjects. Karayanidis, Andrews, Ward, and Michie (1995); however, found a significant decline in hit rate, increase in N1 amplitude, and reduction in $\mathrm{P} 3 \mathrm{x}, \mathrm{P} 3 \mathrm{~b}$, target negativity, and mismatch negativity amplitude in old subjects when tone sequences were dichotically presented. The amplitude and duration of the late Nd component incressed with age, reflecting modulation of both attended and unattended standard ERPS.

\section{Stroop Color Word Test}

Changes in automaticity and interference susceptibility associated with aging can be examined by using the Stroop Color Word Test (Stroop, 1935). From a lifespan study with subjects aged from 7 to 80 years, Comalli, Wapner, and Werner (1962) concluded that interference begins early in the school years, rising to its highest level as reading skills develop. With continuing development of reading, interference declines until approximately age 60, at which point it begins to increase again. This increase in interference after age 60 has been confirmed by others (Cohn, Dustman, \& Bradford, 1984; Panek, Rush, \& Slade, 1984). Houx, Jolles, and Vreeling (1993) found older subjects to perform worse than younger subjects, and furthermore noticed that mild biological or environmental factors, such as repeated general anesthesia, or TBI, can compromise optimal brain functioning, resulting in a significant increase in interference. Hartley (1993) found no evidence for a decrease in selective capacity associated with aging. Hartley used a color-block version in which the color word was adjacent to a color block and a color-word version in which the word was printed in color: An advance cue preceded the stimulus by 100 to 300 milliseconds, indicating where it would appear. Age differences were small on the color-block version and large on the color-word version. Hartley (1993) considered these data to support the hypothesis that the posterior brain attention systems that are responsible for selecting a spatial location are relatively well preserved with advancing age, but that anterior brain attention systems that are responsible for selecting a line of processing are compromised (see also Posner \& Peterson, 1990). It has been suggested that the principal function of the prefrontal cortex is the organization and execution of complex, temporally organized processing sequences (Fuster, 1980; Kolb \& Whishaw, 1990). The effects of age on Stroop performance furthermore appear to be affected by catutiousness, which is manifested more in terms of the accuracy of response than in the speed of response (Rush, Panek, \& Russell, 1987), verbal intelligence (Rush, Panek, \& Russell, 1990), and practice effects (Dulaney \& Rogers, 1994). The latter authors view selective inhibition as an active, time-dependent control process that develops over time and that inhibition is released after the response to the task-appropriate information has been made.

In conclusion, the capacity to select relevant information does not appear to be affected by the process of aging, although several studies suggest that older subjects have a lower capacity to inhibit irrelevant (distractor) information. Madden's (1990a) review of research on age differences in the use of attention during visual search and classification tasks suggests that the selective aspect of attention is relatively resistant to age-related change. The capacity aspect of attention, in the sense of the limited processing resources that underlie task performance, appears to undergo an age-related decline. 


\section{$3.2 .3 \quad$ Divided Aitention}

Divided attention refers to the process by which attention is controlled so that the individual can successfully perform two or more tasks simultaneously (McDowd \& Birren, 1990). Previous research has yielded conflicing pesults regarding the relationship between adult age and the ability to divide attention between two concurtent tasks: At least some of the inconsistency is attributable to methodological variations, such as the manner in which divided attention ability has been assessed, how single-task performance has been considered, and the degree of control over the emphasis placed on exach task.

Based on a review of the divided attention literature in the context of age changes in memory, Crak (1977) concluded that age-related deficits of divided attention oceur in the elderly. Some years later, however, Somberg and Salthouse (1982) challenged the generality of this finding, because they found no significant age difference in divided attention ability after controlling for age differences in single-task performance and after measuring divided attention performance independently of resource allocation strategies. They concluded that previously observed age differences in divided attention performance were artifacts of initial differences in single-task performance. The 1984 study by Salthouse, Rogan, and Prill (1984) suggests that either the total amount of attention available or the efficiency of its allocation decreases with age. They compared the ability of young and old subjects to remember concurrently presented series of letters and digits. Attention-operating characteristics (AOC, Kinchla 1980 ) were derived by varying the attentional emphasis, by means of instructions and pay-offs, across the two types of material under different conditions. Analysis of AOCs revealed that older subjects showed greater performance deficits than younger subjects when they had to divide their attention between the two tasks, even though the difficulty of the two tasks was individually adjusted for each subject. In line with previous findings (Somberg \& Salthouse, 1982), Salthouse and coworkers (1984) did not find that age affected the ability to vary attention between concurrent tasks in response to instructions and pay-offs.

The title of the 1995 manuscript by Salthouse, Fristoe, Lineweaver, and Coon suggests that many of the dual-1ask studies performed since 1982 (Somberg \& Salthouse, 1982) somehow provided litule unsight into the mechanisms underlying divided attention performance. Salthouse and collengues (1995) conducted two experiments in which a speeded decision task was performed during the retention interval of a letter-memory task. One of the major findings was that there were large and significant age-related effects on dual-task performance. When performance on these tasks was corrected for the single-task performance, the dual-task effects were frequently non-significant. It therefore appears to be reasonable to conclude that dual-task performance is mediated by perlormance of the individual constituent tasks. The minor effects of age on dual-task performance could not be explained by a reduced capacity of the central executive responsible for monitoring and controlling concurrent activities, as proposed by Baddeley (e.g., Baddeley, 1986; Baddeley, 1992; Baddeley, Logie, Bressi, Della Sala, \& Spinnler, 1986). Salthouse and colleagues (1995) also found that the effects of complexity were relatively small and concluded that the mechanisms responsible for age-related effects on measures of both simple and complex performance are related to a slower speed of executing elementary operations. As will be shown, however, some studies suggest that in several cognitive task domains elderly people might not aways perform at the same level as their younger counterparts. 


\section{Perceptual-Motor Tasks}

McDowd (1986) presented evidence suggestive of an age-related decline in divided attention performance. She examined the effects of extended practice on divided attention performance by using two perceptual motor tasks under both single- and dual-task conditions across six $\mathbb{l}$-hour sessions. Absolute levels of performance improved reliably over sessions for both young and old subjects, but divided attention performance remained worse in the old subjects. Relative divided attention costs were consistently higher for the older subjects across experimental sessions. These results confirm previous suggestions that older people are vulnerable to the effects of divided attention. By using a perceptual motor task similar to that used by McDowd (1986), McDowd and Craik (1988) investigated the possibility that age differences in dual-task performance could be amplified by increasing the complexity of the constituent tasks. This was achieved by varying the central, cognitive nature of the tasks or the degree of choice involved. They found strong evidence for an age-related decline in divided attention performance that could be attributed to the effects of task complexity and not primarily to difficulty to divide attention in old subjects. This finding led McDowd and Craik (1988) to conclude that the use of divided attention tasks is just another way to increase task complexity.

\section{Tasks Specifically Involving Memory}

Baron and Mattila (1989) also examined the effects of extended practice with a memory scanning procedure in which lists of visual and auditory items were presented singly (singletask condition) or together with a second visual or auditory list (dual-task condition). Identification of test items in young and old men was slower with the dual-task procedure, and experience with the tasks produced only small changes in response latencies. When time limits were placed on responding, latencies decreased substantially, and the difference between single and dual-tasks was reduced. Although the older men were slower throughout the experiment, the time limit contingency reduced age differences in response Jatencies in both tasks. The task effect was greater for the older men, but this age difference also was reduced by the time limit procedures. Lorsbach and Simpson (1988) examined dual-task performance in letter-matching and found that older subjects were especially slower in the late stages of category matching, suggesting that retrieval and comparison of category information are particularly demanding for older adults. This finding is in line with the results of the study by Greenwood and Parasuraman (1991), who also showed that a decreased divided attention performance in older subjects is mainly caused by greater attentional costs for the late stages of information processing, which involve matching and response selection. Early - encoding- and late stages of processing were assessed by using a dual-task paradigm involving a letter-matching task and a probe-response time task. Aging was associated with increased attentional costs of encoding 50 milliseconds after letter presentation but not at later times. This effect was largely due to the very fast encoding of probes by young adults. This effect was present for both young-old and old-old subjects and was not attenuated by practice. The study by Park. Smith, Dudley, and Lafronza (1989), however, showed that the free recall and clustering performance of older subjects in a memory task declined more than that of young subjects when attention was divided at encoling, but not at retrieval. This suggests that aging does not have a general effect, and that the effect of age appears to depend heavily on variations in task and modality characteristics. Using a fame judgment task with either a full attention or divided attention condition, Jennings and Jacoby (1993) found that age and divided attention had a detrimental effect on consciously controlled but not on automatic memory processing. 


\section{Auditory Perception Tasks}

With regard to auditory perception. Tun, Wingfield, and Stine (1991) examined the speech-processing capacity of young and older adults with a dual-task paradigm. Subjects listened to spoken passages for later recall while performing a concurent response time task. The latter task assessed the use of cognitive capacity in the speech memory task. Age differences in decision latencies in the repsonse time task were eliminated by matching subgroups of young and older subjects for working memory span. The results suggest that an age-related reduction in working memory efficiency contributes to age differences in the processing of speech. In another study on speech processing as a function of age, young and old subjects listened to and immediately recalled spoken passages presented at speech rates ranging from 140 to 280 words per minute (Tun, Wingfield, Stine, \& Mecsas, 1992). In a divided attention condition subjects concurrently performed a picture recognition task. Immediate memory performance in old subjects decreased when speech rates were very fast. These findings are consistent with the previously mentioned general slowing hypothesis (Cerella, 1990; Salthouse, 1985). In the divided attention condition, the Age x Speech Rate interaction, however, was not attenuated. This suggests that aging may reduce the rate at which speeech is processed into memory, but this is conceptually distinct from an age-related reduction in attentional capacity. By using their so-called Divided Attention Questionnaire, Tun and Wingfield (1995) found that self-perception of ability in old age varied with task domain, such that activities that involved monitoring of novel information became increasingly difficult with advanced age, while routine activities and those involving speech processing showed little change across the older groups studied. Tun and Wingfield proposed that beliefs about self-efficacy under conditions of divided attention can have important consequences for everyday life functioning of the elderly.

\section{Tracking Tasks}

A possible decline in divided attention might be detected in a simulated driving task. Ponds, Brouwer, and Van Wolffelaar (1988) examined young, middle-aged, and old subjects in two continuous performance tasks. The first task consisted of compensatory tracking, which was modelled on the everyday life activity of car driving, whereas the second task involved a self-paced visual choice response time task requiring analysis of a visual display presented so that no eye movements were required when both tasks were performed simultaneously. Results showed a divided attention deficit in old subjects compared to young and middle-aged subjects, who did not differ in their ability to divide attention. These findings were replicated by Brouwer, Waterink, Van Wolffelaar, and Rothengatter (1991) in a slightly modified experimental seiting, in which they tested young and old experienced and active drivers. Again, the first task was a compensatory lane-tracking task involving a three-dimensional road display, The second task was a timed, self-paced visual analysis task involving either a vocal or manual binary response to dot patterns projected within the road display. Single-task difficulty was individually adjusted for each subject. To control for individual differences in attention allocation strategy, the dual-task was performed according to three different sets of instructions based on the relative importance of each task. Compared with young adults, older adults showed a significantly decreased ability to divide attention. This effect was apparent in lane tracking and in the accuracy of visual analysis. The impairment of divided attention was less pronounced for the vocal response than for the manual response. This suggests that difficulty in integrating responses may be an important determinant of poor dual-task performance in old 
age. However, not all dual-task conditions necessarily lead to a disproportional decrease in the performance of the elderly. Wickens, Braune, and Stokes (1987), for instance, found a monotonic decrease in processing speed with age but no difference in time sharing abilities between age groups. They used a tracking task performed alone and concurrently with different versions of a Sternberg memory search task (Sternberg, 1969) that varied the degree of resource competition with the tracking task.

Parasuraman and Nestor (1991) suggested that motor vehicle accident rates are related to deficits in switching attention (see next paragraph) and less to deficits in divided or sustained attention. They found that many attentional functions, particularly the switching of attention, were impaired in the early stages of Alzheimer's disease and may thus contribute to an increased accident risk. It is unclear, however, whether accident risk is related to attentional switching between stimulus sources per se (i.e., location switching) or to a more general ability to switch "stimulus set" between features, concepts, or rules. McKenna, Duncan, and Brown (1986) found no evidence for the latter. Crook, West, and Larrabee (1993) found an age-related decrease in performance in both verbal recall tasks and response time tasks in a computer simulation where subject of different ages had to perform simultaneous tasks related to car driving. Changes in response time were primarily due to cognitive rather than psychomotor factors. They suggested that a driving response time test as an ecological assessment of dualtask performance should be included in all test batteries used to assess the cognitive performance of elderly people.

\section{Conclusion}

Age differences in dual-task performance can be interpreted as a consequence of an increase in overall task complexity rather than as a result of the need to divide attention. Crossley and Hiscock (1992) found a heightened vulnerability of old subjects to concurrent task performance when subjects had to perform a concurrent tapping task in addition to tasks that varied in complexity. They did not attribute this age-related decline to general slowing or to diminution of a specific resource, but rather suggested that a general-purpose processing resource might be diminished with increasing age. Pashler (1994) questioned the usefulness of widely accepted concepts such as attentional resources and probe response time methodologies. Studies of the psychological refractory period effect indicate that there is a stubborn bottleneck involving the process of choosing actions and probably memory retrieval generally, together with certain other cognitive operations. Other limitations associated with task preparation, sensory-perceptual processes, and timing can generate additional and distinct forms of interference. These studies also suggest new ways of thinking about continuous dual-task performance, effects of extraneous stimulation (e.g., stop signals), and automaticity.

In conclusion, at least some aspects of divided attention efficiency appear to decline with age, but this conclusion heavily depends on the kind of task that is used to measure effects of age on divided attention. The general conclusion might be that the capacity aspect plays an important role in divided attention performance, as is also the case in selective attention and switching attention. As already stated, older subjects appear to be particularly susceptible to the effects of task complexity and tend to have an inferior performance on these tasks. When divided attention tasks are considered as a way of increasing task complexity, as suggested by McDowd and Craik (1988), we can conclude that the division of attention per se is not affected in old age. 
Shifting attention can be described as the process of alternately monitoring two or more sources of input (McDowd \& Biren, 1990). Intwitively, this definition appears to be very similar to the one that describes divided attention. The main feature of shifting attention, however, is that this process essentially involves serial processing, whereas divided attention demands the parallel processing of several inputs. When a subject, for instance, is driving a car, he has to shift his attention to road signs and other road users from time to time. When, however, he arrives at a roundabout, he has to divide his attention over a vast array of wisual inputs in order to cope effectively with the driving task demands at hand. Shifting attention requires endogenous and exogenous initiated shifts of attention, of which the latter can be characterized as being more automatic and reflexive than the former (Jonides, Naveh Benjamin, \& Palmer, 1985).

Folk and Hoyer (1992) examimed age differences in the efficiency of attentional shifts in a spatial cuing task in which abruptly presented peripheral cues or central, symbolic cues were presented prior to a target stimulus at intervals ranging from 50 to 250 milliseconds. No age differences were found in the magnitude of cuing effects with peripheral cues. Similar results were obtained with symbolic cues, although cue difficulty affected the cuing effects in old subjects. Increasing age thus appears to affect cue encoding, but not the efficiency of the shift process. It must be stated, however, that the controlled allocation of attention in response to a peripheral cue involves qualitatively different mechanisms from those associated with the endogenous generation of an expectancy (Klein, 1994). Ward (1994) furthermore found that involuntary stimulus-driven attention shifts might be controlled by a modality-specific mechanism for visual tasks, whereas stimulus-driven shifts of auditory attention are controlled by a supramodal mechanism. He found that visual precuing affected response time to localize both visual targets and auditory targets, but that auditory precues affected only the time to localize auditory targets. Moreover, when visual and auditory cues conflicted, vistial cues dominated in the visual task but were dominated by auditory cues in the auditory task.

With regard to cognitive aging, Hartley and McKenzie (1991) confirmed the hypothesis of Cerella (1985a) that age differences in attention may in fact reflect age differences in perceptual processing of stimuli falling outside the fovea. In addition, Hartley and McKenzie (1991) showed that there were greater age differences when attention had to be narrowly focused, indicating that attentional effects can be separated from the effects reported by Cerellat. Furthermore, they showed that age differences in extrafoveal perception could be removed by increasing the duration of trrget presentation from 200 to 2000 milliseconds, suggesting that the perceptual deficits of older adults are due to slower processing of stimuli outside the fovea. The study by Madden, Connelly, and Pierce (1994) suggested that young and old subjects are equally well able to shift their focused attention between display locations with target-only displays and $40 \%$ cue validity. With a $70 \%$ cue validity and the presence of distractors, increased age negatively affected the attention shifting time. The presence of distractors thus appears to compromise the performance of older subjects, as is also the case in selective attention performance. These findings are in line with the model of Hasher and Zacks (1988) who consider the distraction by irrelevant stimuli to be caused by a lack of inhibitory control. An explanation in terms of perceptual noise (Allen et al., 1992) is not very likely, since target
and distractor items were visually dissimilar. 


\section{$32.5 \quad$ Sustained Attention}

Sustained attention or vigilance is involved in the effictent detection of infrequent and unpredictable events, particularly after prolonged periods of observation. Sustained performance may be important in many practical situations, including radar and sonar monitoring, industrial inspection, process control, and car driving. Because age has been shown to influence both alertness and effortful control processes (e.g., Kausler, 1982), the study of the effect of age on sustained attention (or the lack thereof) presents an opportunity to investigate maturational changes in their simultaneous interplay. In the laboratory, vigilance tasks have provided the fundamental experimental paradigm for the investigation of this phenomenon (Jerison, 1977). Much research has focused on the decrease in vigilance - the significant decline during the period of work in the number of signalls correctly reportedwhich occurs in most, though not all, laboratory monitoring tasks. Sustaned attention performance has usually been evaluated by measuring target detection accuracy to correct detections, and the false alarm rates. There has been some debate over the extent to which this decrease in vigilance occurs outside the laboratory (Mackie, 1987). However, there appears to be sufficient evidence that loss of vigilance can be a problem in real-world situations, and provided that task parameters are comparable, the monitoring of performance in laboratory and operational settings reveals strong similarities between the two (Craig, 1984; Davies \& Parasuraman, 1982; Parasuraman et al, 1987).

With regard to the effects of age on sustained attention, results are rather mixed. The review by Davies and Parasuraman (1982) showed effects of age on any measure of vigilance behavior in no more than half of 13 studies. When significant age effects were reported, subjects older than 60 years of age responded more slowly, detected fewer targets, and made more false alarms on cognitive tasks with a memory component. Davies and Parasuraman (1982) concluded that the overall level of vigilance is directly related to the level of arousall. Surwillo and Quilter (1965a, 1965b) reported a direct relation between arousal levels and the likelihood of detecting a target and a decrease in arousal level in increasingly older age groups. This would imply that arousal and vigilance decrease with increasing age and that decreased vigilance is a direct result of reduced overall levels of arousal in the elderly. Mathews, Davies, and Holley (1990) suggest that arousal increases the availability of resources for sustained attention. They found that a higher self-reported arousal consistently facilitated those sensory and simultaneous cognitive tasks that showed a significant decrease in sensitivity. Other studies not reviewed by Davies and Parasuraman (1982) also yielded mixed results. The contradicting results might in part be caused by the wide variation in the age interval of subjects assigned to the "old" group. Gridley, Mack, and Gilmore (1986), for instance, administered a non-verbal auditory sustained atteintion test to young and old women and found older subjects to be as accurate and as fast as young women.

A typical task used to measure sustained attention is the Mackworth clock test, which requires subjects to identify double-time movements of a watch hand around the face of a clock. Giambra and Quilter (1988) found no age differences or changes in detection accuracy on this 62-minute sensory vigilance task. Response time to targets showed significant age-related 18-year longitudinal changes and one significant cross-sectional age difference. The overalll age effect displayed a U-shaped function with a minimum at middle age. Age differences and changes were detected by using the skin potential response latency as a measure of arousal. The overall age effect was also consistent with a $\mathbb{U}$-shaped function. Giambra and Quilter found little 
consistent evidence of a differential effect of age on the decrease in vigilance. Earlier studies demonstrated age effects (Quilter \& Giambra, 1983: Surwilla \& Quilter, 1964), but these results were most likely due to a statistical error of the first type (Giambra \& Quiter, 1988). Giambira, Quilter, Phillips, and Hiscock (1988) furthermore showed that age was not related to the strategy used, but that the strategy used was significantly related to target detection accuracy. Strategies based upon active mental involvement produced the best target detection in the vigilance task; a strategy based only on self-arousal manipulation through himited physical movement produced the worst target detection. From the same dataset Giambra and Quilter (1989) observed sex differences in target response time and on measures of autonomic arousal; women were slower and less aroused than men. Sex differences in the time course of vigilance performance were inconsistent, and age did not significantly moderate the sex differences in the decrease in wigulance. Parasuraman, Nestor, and Greenwood (1989) demonstrated a decline in perceptual sensitivity (d.') when stimuli were highly degraded in a high-event rate digit-discrimination vigilance task. However, both young and old subjects showed a similar sensitivity when stimulus degradation was moderate, and a similar decrease in sensitivity over time when stimulus degradation was high. These authors also concluded that the process of sustained allocation of capacity - as reflected in temporal changes in sensitivity - operates similarly in young and older adults. Apart from event rate and stimulus degradation, spatial uncertainty in a cognitive vigilance task requiring identification of a target, could also negatively affect performance in older subjects. Myerson, Hale, Wagstaf, Poon, and Smith (1990) found that an increased spatial uncertainty decreased the target detection rate and $d^{\prime}$ to a greater extent in older subjects than in young subjects. No age differences were obtained for a low-spatial-uncertainty condition. They also found that the decrease in vigilance was magnified when the event rate was high and when spatial uncertainty was high. They suggested that cognitive vigilance is sensitive to age when demands on visual attention capacity are increased by a high event rate or spatial uncertainty. Deaton and Parasuraman (1993) compared sensory and cognitive vigilance as a function of age and event rate by using tasks with the same stimuli (digits) equated for presession performance levels and which differed only in the type of discrimination required for target detection. Over the course of 32 minutes, the detection rate for the sensory task showed the normal decrease in vigilance, whereas the detection rate for cognitive task performance remained stable. The decrease in hit rate with an increase in event rate was more pronounced for the cognitive than for the sensory task. Older adults had lower detection rates for both tasks and higher false alarm rates for the sensory task, but not for the cognitive task.

\subsubsection{Factors Affecting Attentional Performance}

In research into aging and attention, as is the case in all research on attention, more progress has been made at the empirical level than at the theoretical level. It is possible to predict reasonably accurately whether or not two tasks can be combined successfully, but the accounts offered by different theorists are very diverse. Accordingly, we will make a start by discussing the two major factors that may affect attentional performance associated with aging. These factors - task complexity and experience-clearly do not affect performance in all attentional domains to the same extent. In this sense they should be considered as possibly influential background
variables. 


\section{Task Complexity}

Intuitively, one would expect subjects to have a less than optimal performance when tasks aro more complex. When examinining attentional functions in relation to aging, one should try to separate the effects of task complexity from the effects of aging. If elderly people are more vulnerable to changes in task complexity and if they would perform inferior to have a poorer performance than their younger counterparts on attentional tasks, we would expect to find a disproportional decrease in attentional function in older subjects. It is important to study such effects, since they can provide insight into the mechanisms that contribute to a decreased attentional performance in the elderly. Task complexity can, for instance, be augmented by increasing the mental load or by decreasing the perceptual difference between targets and distractors. In the last case, we would expect older subjects to perform worse than younger subjects (Duncan \& Humphreys, 1989). Although Salthouse and coworkers (1995) stated that complexity effects - even on single-task performance-are virtually non-existent, a few studies suggest that complexity modulates the effects of age.

With regard to language processing, Ulatowska, Hayashi, Cannito, and Fleming (1986) studied the effects of age on narrative and procedural discourse at various levels of complexity and stimulus/response requirements and found a significant ambiguity of reference in the old middle-aged subjects in comparison to middle-aged subjects, which increased markedly in older subjects. The extent of the impairment increased with the complexity of the task. In 1995 Fitzgibbons and Gordon Salant examined the combined effects of aging, hearing loss, and stimulus complexity on duration discrimination and found elderly listeners to perform worse with increasing stimulus complexity. Hearing loss had no systematic effect on discrimination performance.

The poorer performance of older subjects on complex relational tasks may be due to inadequate relational structures for the integration of problem elements (Salthouse \& Prill, 1987). In series completion tests, Salthouse and Prill found that young and old adults did not differ significantly in the effectiveness of inductive reasoning when determining simple relations, but old adults had an impaired performance when the relations were complex.

With regard to motor performance, the 1988 study of SteImach, Amrheitu, and Goggin (1988) in which old and young subjects who were asked to make unimanual, bimanual symmetric, and bimanual asymmetric movements, showed that in addition to an overall increase in latency, old subjects exhibited a linear increase in response initiation with increasing task complexity similar to that of the young group. Moreover, the old subjects showed an increased response execution latency. Compared to young subjects, old subjects showed a greater asynchrony in response initiation of bimanual movements and in response termination. In a dual-task consisting of two unrelated one-dimensional compensatory tracking tasks, Korteling (1993) found that older subjects are penalized when there is incompatibility between control and display integration. The dissimilarity effects were the greatest in old subjects when partial integration involved integration of the response component.

Within the auditory domain, Fitzgibbons and Gordon Salant (1995) studied the age-ralated changes in temporal processing. They measured the discrimination levels for signal duration using simple and complex stimuli and showed that the effects of stimulus complexity on discrimination were the greatest among old listeners. The difficulty elderly listeners had in discriminating duration changes even in simple sounds suggests that there are age-related changes in central timing mechanisms. 


\section{Experience and skill Developnent}

Apart from looking for factors that may affect cognitive performance in a negative way - such as lask complexity - cognitive aging research is also trying to discover factors that might contribute to successful cognitive aging. One of these potentally beneficial factors may be the experience and skill that people acquire throughout life and which may serve them well in later life. Indeed, several studies indicate that a high level of skill partly counteracts the negative effects of cognitive aging. Jordan and Rabbitt (1977), for instance, found an increase in differential slowing in performance between young and old an Age $\mathrm{x}$ Task Complexity interaction with increasing complexity of perceptual response mapping. This phenomenon disappeared with practice, leaving an apparent age lag constant, which they thought to be caused by an increased central processing time rather than peripheral factors. Older subjects might even show a stronger inerease in performance than younger subjects in learning and retaining a novel motor skill, as was demonstrated by Anshel (1978). When older subjects are involved in stressful competitive activities, like miniature golf, the deterioration in motor performance observed in older players may be due to age-related changes in attentional functioning, as was suggested by Molander and Backman (1989).

Skill development, however, may not counteract the negative effects of aging on all cognitive domains. Hashtroudi, Chrosniak, and Schwartz (1991), for instance, examined the effects of aging on two kinds of implicit memory tasks: repetition priming and skill learning. Skill learning of reading inverted words was impared under data-limited conditions, whereas priming was unaffected under these conditions. However, when older adults were presented with more perceptual information than were young adults, the age deficit in skill learning was eliminated. These authors proposed that the demonstrated age deficit in skill learning is related to a deficit in pereeptual (re)organization. With regard to memory span capacity, Taub (1973) found that young subjects benefitted more from repeated sessions than did aged individuals. Korteling (1991) showed that skill integration could decrease the negative effect of visual competition in dual-task performance, but only in young subjects.

The suggestion of Hashtroudi and associates (1991) that age-related deficits in skill learning might be related to a perceptual shortage is supported by Rogers and Fisk (1991). In a visual search task, they showed that the pattern of data during training and at reversal supports the proposal that age differences in extended practice visual search are due to differences in the ability to differentially strengthen targets and distractors. Another study by Fisk and Rogers (1991) suggested that the dissociation of the pattern of the effects of age in memory and visual search involves different processing mechanisms for memory search and in visual search. "They furthermore hypothesized that age-related differences in automatization do not occur in memory search tasks. This notion is at odds with the findings of Strayer and Kramer (1994), who tested the hypothesis that a conservative response bias in older persons interferes with the acquisition and mastery of the cognitive skills neededl to perform consistent mapping (CM) and varied mapping (VM) versions of a memory search task. Older subjects had a less skilled performance than younger subjects. Strayer and Kramer (1994) attributed this to both age-related differences in the rate of associative learning and differences in asymptotic levels of performance. In 1994 , however, Rogers, Bertus, and Gilbert (1994) found further evidence for their hypothesis that age-related differences in automatization do not occur in memory search tasks. They assessed dual-task performance in young and old adults after extensive single-task practice on $\mathrm{CM}$ and VM search tasks. Next, subjects practised the dual-tasks, VM visual search plus VM memory search and $\mathrm{CM}$ visual search phus VM memory search. In the condition of varied visual search 
plus waried memory search, young and old aduits showed similar dual-task deerements. These results suggest that practice may play an important role in determining age-related dual-task differences. In the consistent-waried condition, young adults attaned single-task performance levels, because they had automatized the consistent task, which was not the case in old adalts, who were unable to match their single-task performance levels. In a $\mathrm{CM}$ memory search task where one of the search conditions was subject to interfering processing activity prior to the retention interval, Fisk, Cooper, Hertzog, and Anderson Garlach (1995) showed that older adults are more vulnerable to the effects of interference, even on tasks that have been extensively rehearsed. Old and young adults retained task-specific skills equally, although old adults ${ }^{\prime}$ performance cleclined more than young adults' performance for trained CM stimuli. When an interfering processing activity was inserted prior to the retention interwal; old adults performance declined disproportionally more than young adults' performance, especially when compared to the task not subjected to such interference. For both old and young adults all initial retention deficits were quickly eliminated within retention retraining.

The suggestion of Rogers and colleagues (1994) that practice may play an important role in determining age-related differences in dual-task performance may not apply to all dual-task performance. In a study by McDowd (1986), young and old adults performed two perceptual-motor tasks under both single- and dual-task conditions across six sessions. She found that the absolute levels of performance improved reliably over sessions for both young and old subjects, but divided attention performance remained worse in the old subjects. Holland and Rabbitt (1994) furthermore suggested that vigilance, speed and distance judgements, and coordination in car driving are intrinsically difficult to attain for older people. Vigilance performance could to some extent be influenced by practice, although the decrease in vigilance in a 30 -minute vigilance task at low and high event rates for 20 sessions could not be eliminated completely (Parasuraman \& Giambra, 1991).

The specification of attention in terms of anatomy and function can be useful in clarifying the underlying bases of attentional disorders. In particular, many of these disorders affect cortical areas, with reciprocal subcortical transmitter network interactions. Recent brain imaging (Raichle, 1987) and neurophysiological techniques (Colby, 1991) showed that attention is neither a property of a single brain area, nor of the entire brain (Mesulam, 1990; Posner \& Peterson, 1990). In humans and monkeys, at least three systems appear to be involved in attentional performance: (1) the orienting circuit, (2) the executive circuit, and (3) the alerting circuit.

The orienting circuit consists of the posterior parietal lobe, the superior colliculus, and the thalamic pulvinar nucleus (Posner, 1995). Brain injury to any of these areas is associated with a reduced ability to shift attention. Each of these areas is affected differently by lesions. Attention-related enhancement of visual responses in the posterior parietal cortex may be a neural substrate for shifts in visuospatial attention (Goldberg \& Segraves, 1987). The right parietal lobe is dominant for shifts in spatial attention and controls attention for both visual fields, whereas the left parietal lobe appears to play a subsidiary role (Corbetta, Miezin, Dobmeyer, Shulman, \& Petersen, 1991). Corbetta, Miezin, Shulman, and Petersen (1993) furthermore pointed at a possible involvement of the superior frontal cortex in shifts to peripheral locations. The difference in dominance might be related to differences in levels of 
arowsal, as was suggested by Posner and Peterson (1990). Lesions in the superior colliculus cause a slowing in the ability to shift attention, while lesions in the pulvinar nucleus also cause difficulty in covert orienting (Posiner, 1988).

The frontal cortex appears to be involved in the executive circuit. Thus; tasks requiring supervisory control (Duncan, Burgess, \& Emslie, 1995; Shallice, 1988; Shallice \& Burgess, 1991 a; shallice \& Burgess, 19916 ) are severely affected by lesions of the frontal lobes. The executive circuit primarily involves the anterior cingulate gyrus, which is closely connected with the dorsolateral frontal cortex (Frontal Eye Fields, Area 8) and the posterior parietal cortex (Goldman-Rakic, 1988). The prefrontal and posterior parietal cortices have reciprocal interconnections not only with each other, but also with an identical set of additional cortical areas, supporting an architecture of connectivity consistent with parallel processing (Morecraft, Geula, \& Mesulam, 1993). The anterior cingulate gyrus appears to be involved in a wide range of activities that have been termed collectively 'executive functions' (Vogt, Finch, \& Olson, 1992). Raichle and colleagues (1994) showed the anterior cingulate gyrus to be active when the Supervisor System is needed for appropriate behavior in the Norman-Shallice model (Norman \& Shallice, 1986). Likewise, the anterior cingulate gyrus is inactive when the Supervisory System is inactive and the contention scheduling mechanisms are active. The posterior cingulate cortex is known to contain neurons that monitor eye movements and that respond to sensory stimuli. These connections are suggestive of an integrative mechanism, since the lateral frontal cortex appears to be involved in semantic processing, while the posterior parietal lobe is important for spatial attention. Damage to one of these areas induces physiological dysfunction in the other (Mesulam, 1994). Funahashi, Chafee, and Goldman-Rakic (1993), and Funahashi and Kubota (1994) furthermore suggest that lateral frontal cortex neurons code for the location of the visual stimulus in working memory, and that working memory can inhibit as well as facilitate responses.

The third brain circuit involved in the attentional process is the alerting circuit. This circuit appears to involve the right frontal area, since patients with a lesion in this area have difficulty staying alert for prolonged periods (Posner \& Peterson, 1990). In addition, Pardo, Pardo, Janer, and Raichle (1990) also showed a parietal involvement in sustained attention performance. An additional contribution is provided by a set of diverse projections to all three attentional circuits from brain stem and thalamic components of the reticular activating system. It has been suggested that this input plays a role in the modification of the activation bias (or level of arousal) in each of the major areas (Mesulam, 1990).

In conclusion, selective attention is thought to be organized at the level of a extensive system that contains three components or local circuits, each providing a slightly different coordinate system for mapping the environment. The posterior parietal component provides a sensory representation of extrapersonal space and disengages attention from its presents focus, the frontal component provides a map for the distribution of orienting and exploratory movements, and the cingulate component provides a map for assigning value to spatial coordinates. The superior colliculus is closely related to the motor component and is active when one focuses attention on a target, whereas the pulvinar nucleus and striatum are associated with all three cortical components. The pulvinar nucleus furthermore selects the information that is relevant for further processing. 
Aftention should be recognized as a prerequisite for optimal cognitive performance. When attentional performance declines with age, we can expect that other cogmitive functions will decline likewise. Stankov (1988), for instance, showed that age-related changes in fluid and crystallized intelligence are dependent on changes in attentional processes, especially attention switching, sustained attention, and perceptual speed. A major difficulty, however, is that specific terms like attention switching and vigilance performance are often used in different ways in attentional aging research. A general agreement on which attentional domains should be distinguished is also lacking. The various aspects of attention mentioned in this chapter, however, largely coincide with the opinio communis about which aspects of attention should at least be distinguished. These studies provide evidence that some aspects of attention tend to decline with advancing age.

The issue of what happens to unattended stimuli has been investigated for the visual and auditory systems. These studies suggest that there is some processing of unattended stimuli, with the amount of processing varying as a function of how easy it is to discriminate between targets and distractors. Older subjects tend to process non-relevant information much more than younger subjects when the distinction between targets and distractors is smaller. "The ability to select relevant information itself does not appear to be affected by age, but there rather appears to be a decrease in the availability of processing resources for this cognitive operation. When a subject has to monitor two different sources of input alternately, aging appears to affect encoding, but not the efficiency of the shifting process itself. Studies of divided attention involved presenting subjects with two tasks at the same time, with instructions to perform both tasks as well as possible. As is the case in selective attention, the divided attention performance of older subjects appeared to be affected by task similarity. Task difficulty and practice also appeared to be important factors in predicting the age-related divided attention performance. Divided attention tasks can be regarded as a means to increase task complexity, which results in a decreased performance in the elderly. The extent to which deterioration takes place largely appears to depend on the methodology and the model that is used to explain these age-related differences. Findings with regard to vigilance performance suggest that the effect of adult age on vigilance also depends on the type of task studied and the aspect of performance examined. Age differences in perceptual and cognitive performance are often most apparent when relatively greater demands on processing capacity are imposed on the subject. Thus, age differences in the rate of the decrease in vigilance over time, and in perceptual sensitivity, should be more apparent under conditions in which sensitivity also decreases. However, in most previous studies the processing capacity demands of the vigilance task used were relatively low.

These findings should be considered with some caution, since most attentional research has been based on the notion that there is a unitary, limited-capacity, attentional system. As far as focused attention is concerned, the limitations of this system produce bottlenecks in processing. As far as divided attention is concerned, attentional limitations often prevent the successful performance of two tasks at the same time, and lead to the development of automatic processes do not depend on attentional capacity. The suggestion that there may be more than one attentional system has been substantiated by Posner and Peterson (1990), who icentified at least three different attentional processes: disenagegement of attention from a stimulus; shifting of attention from one stimulus to another; and engagement of attention on a new stimulus. If we accept that there is more than one attentional process, the distinction between automatic and 
controlled processing does not provide insight into the attentional processes affected by aging. Another topic of concern is the fact that the functions of attention in most attentional theories. tend to receive litte considetation. In experimental settings, attention is directed by instructions, whereas in the real world atterntion is strongly directed by the motivational state and by the goals that subjects are pursuing. Allport (1993) suggests three issues of major importance to be incorporated in future research: a) The segmentation of different streams of parallel information processing; b) The priority that the subject assigns to different goals; and c) The coordination between sensory lnput and action in attentional performance (i.e., the selection for action).

Since the information that people have to deal with in their daily lives is increasing in density and complexity, future research should more than ever focus on the practical relevance of current knowledge of attentional aging and should try to apply this knowledge to real life situations, thus providing an environment that better meets the needs of the elderly.

References

Allen, $P_{*}$ A, Madden, D. J., Groh, K. E., \& Crozier, L. C. (1992). Impact of age, redundancy, and perceptual noise on wisual search. Nowrialis of Genontology: Psychological Sciences, $7(2), 69-74$.

Allport, D. A. (1993). Altention and control: Have we been asking the wrong questions? A critical review of wenty five years. In D. E. Meyer \& $\mathrm{S}$. Kormblum (Eds.), Artenton and Performance, (Vol. XIV. DP. 18.21). Hondon: MIT Pross.

Allpot, D. A. (1980). Auention and performance. In $\mathrm{G}$. Clakton (Exi), Cognitive Psychology: New Directions, (pp. 112-153). London: Roulledge and Kegan Paul.

Ansthel, M. H. (1978). Effect of aging on acquisition and short-term ratention of a motor skill perceptuat and Motor Skills, 47(3), 993.994.

Baddeley, A. (1986). Working Memory. London: Oxford University Press.

Baddeley, A. (1992). Working Memory. Science, 255(5044), 556-559.

Baddeley, A., Logie, R., Bressi, S., Della Sala, S., \& Spinnler, H. (1986). Dementia and working memory. Quanterly Jownal of Experimental Pyychology: Human Experimental Psychology, 38(4),603-618. Baddeley, A., We Wiskantz, 1 . (1993). Atention. Selection, Awareness and Comrol. Oxford: Oxford Unversity
Press. Barnes, J. (1984). The Complete Works of Aristolle, (Vol. 1. pp. 732). Princeton. NJ: Princeton University
Press.

Baron, A., Mathia, W. R. (1989). Response slowing of older adults: Effeets of time-limit contingencies on singlem and dual task performances. prochology and Aging. 4(1), 66.72. Burti, R. A, \& Ciambra, L. M. (1990), Agenrelated dectement in auditory selective attention. Psychology and
Aging. $5(4), 597-599$.

Bowimick, J. (1981). Neuropsychology of Aging. In S. B. Filskov \& T. I. Boll (Eds.), Handbook of Clinical Neuropsychology, (pp. 135-171). New York: John Willey.

Broadbent, D. Q (1958). Perception and Commumication. New York: Pergamon Press.

Brondbenc, D. E. (1971). Dectsion and Stress. New York: Academic Press.

Brouwer; W. H., Walerink, W., Van Wolffelatr, P. C. \& Rothengatler, J. A. (199). Divided attention in experienced young and older drivers: Lane tracking and wisual analysis in a dynamic driving simulator. Human Factors, $38(5), 573-582$

Cerelli, I. (1990). Aging and information-processing rate. In J. E Birren \& K. W. Schaie (Eds.), Handbook of the Psychology of Aging. (3rd ed., pp. 201-221). New York: Academic Press. Christie, J, \& Kleir, $\mathrm{K}$. (1995). Fannilianty and attention: Does what we know affect what we notice? Memory
and Cogminon, $23(5), 547-550$.

Cohen, J. D., Servan, S. D., McClelland, J. L. (1992). A parallel distributed processing approach to 239-269.
. Special Issue: Vicws and varieties of autonaticity. American foumat of prychology, $105(2)$, Cohn, N. B., Dustman, R. E., Bradford, D. C. (1984). Age-related decrements in Stroop Color Test
performance. Jomanal of Chica Psychology, 40(5). $1244-1250$. 


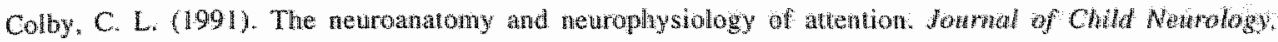
$6($ Suppl. $), 90-118$.

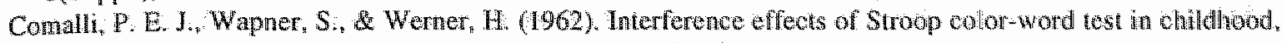
adulthood and aging. Joumal of Genetic Psychology. 10o(1), $47-53$.

Corbeta, M., Miezin, F. M. Dobneyer, S., Shulman. G. L., a Petersen. S. B. (1991). Solwetive and divided attemton during visual discrimination of shape, color, and sped: Functional anatomy by positron emission tomography. Jounat of Newroscience, $1 /(8), 23832402$

Corbetta, M. Miezin, F. M., Shulmar, G. L., \& Petersen, S. E. (1993). A PEI sudy of visuospatial atention. Joumal of Netroscience, $13(3), 1202-1226$.

Craig, A. (1984). Human engineering. The control of vigilance ln $\mathrm{J}$. S. Warm (Ed.). Surtained Antention in Howan Performance, (pp. 247-291). Chichester: John Wiley.

Craik. F. I. M. (1977). Age differences in human memory. In J, E. Biren \& K. W. Schato (Eds.), Hambook of the Psychology of Aging. (pp. 384-420). New York. Van Nostrand Reinhold.

Cranford. J. L., \& Martin, D. R. (1991). Agerelated changes ha binaural processing: I. Evoked potentut findings. American Jownal of Otology, 12(5), 357-364.

Crook, T. H. West, R. L. L Larrabe, O. J. (1993). The driving-reaction time test: Assessing age declines in duallask performance. Developmental Neuropsychology. $9(1), 31-39$.

Crossley, M. \& Hiscock, M. (1992). Agewrated differences in concurrent-task performance of normal adults: Evidence for a decline in processing resources. Psychotogy and Aging, 7(4), 499-506.

Dagenbach, D., \& Cart, T. H. (1994). mhibitory Processes in Artention, Memory, and Language. San Diego: Academic Press.

Davies, D. R., Parasuraman, R. (1982). The Psychology of Vighance. London: Academic Press.

Deaton, J. E., \& Parasuraman, R. (1993). Sensory and cognitive wigilance: Effects of age on performance and subjectiwe workload. Human Perfomance, $6(1), 71.97$.

Dulaney, C. L., \& Rogers, W. A. (1994). Mechanisms underlying reduction in Stroop interference with practice for young and old adults. Jound of Experimenal Pyehology: Laming, Memory, and Cognition, 20(2), $470-484$.

Duncan, J., Burgess, P., \& Emslie, H. (1995): Fuid intelligence after frontall lobe lesiori. Newropsychologia, $33(3), 261-268$.

Duncan, J., \& Humplareys, G. W. (1989). Visual search and stimulus similarity. Psychological Review; $96(3)$, 433-458.

Fisk, A. D., Cooper, B. P.. Hertzog, C., \& Anderson Garlach, M. (1995). Agerelated retention of skilled memory search: Examination of associative leaming, interference, and task-specific skills. Joumals of Gerontalagy: Psychological Sciences, $50(3), 150-161$

Fisk, A. D., \& Rogers, W. A. (1991). Toward an understanding of age-related memory and wisual scarch effects. Joumal of Experimental Psychology: Geneval, 120(2), 131-149.

Fisk, A. D. \& Scerbon M. W. (1987). Automatic and control processing approach to interpreting vigilatice perlormance: A review and rovaluaton. Special lssue: Vigilance: Hasic and applied reserdeh. Hunan Factors. $29(6), 653-660$.

Fitzgibbons, P. J., \& Gordon Salant, S. (1995). Age effects on duration discrimination with simple and complex. stimuli. Jounal of the Acoltitical Soctety of America, $98(6), 3140-3145$.

Folk, C. L.. Hoyor, W. J. (1992). Aging and shifts of wisulal spatial attention. Psychology and Aging, 7(3) " 453-465.

Fullerton, A. M., Smith, A. D. (1980). Age-related differences in the use of redundancy. Jourmal of Geromology, 35(5), 729-735.

Funahashi, S., Chafee, M. V. \& Goldnan-Rakic, P. S. (1993). Prefrontal neuronal actiwity in rhesus monkeys performing a delayed anti-saccade task. Natwre, 365(6448), 753.756.

Funahashi, S., \& Kubota, K. (1994). Working memory and prefontal contex. Newrosetence Research, 21(1), 111.

Fuster, I. M. (1980). The Prefronfal Contex. New York: Raven Pless.

Giambra, L. M. \& Quilter, R. E. (1988). Sustained attention in adulthood: A unique, large-sample, longitudinal and multicohort analysis using the Mackworth Clock-'Test. Psychology and Aging, 3(1) 75-83.

Giambra. L. M., Q Quilter, R. E. (19,9). Sex differences in susianed attention kcross the aduld life span. Journal of Applied Psychology, 7.4(1), 91-95.

Giambra, L. M. Quiltar, R. E.,Phillips, P. B., \& Hiscock, B. S. (1988). Performance on a sustained attention task as a function of strategy: A cross-isectional inwestigation wsing the Mackworth clock- lidet. Bulletin of the Psychonomic Sociery, 26(4), 333-335. 
Goldberg. M. E. Segraves. M. A. (1987). Visuospatial and motor atention the monkey. Special Issue: Selectue visual attention. Newropsychogio, 25(1), 107-118.

Goldman Rakic, P. S. (1988). Topography or cognition: Parallel distributed networks in primate association

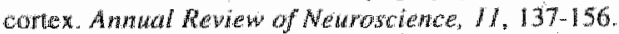

Green wod, P. M. Parsuman, (1991). Effects of aging on the speed and attentional cost of cognitive operations. Developmental Neuropsychology, $7(4), 421-434$.

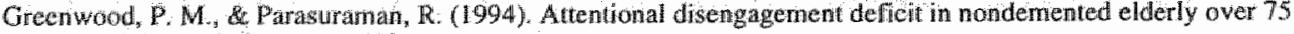
years of age. Aghg ond Cognition, $7(3), 188-202$.

Gridley, M. C., Mack, J. L., \& Cinmore, G. C (1986). Age effects on a nonwerbal auditory sustaned attention task. Penceptwal and Motor Skits, 62(3), $911-917$

Hadden, A. W. (1912), St. Augustine"s "On the trinity". In B. Rand (Ed.), The Clasighal Psychologists. Baston: Hougthton Miffin.

Harley, A, A. (1992). Atention. In F, I. M. Craik \& T. A. Salthouse (Eds.), Handbook of Aging and Cognirion, (pp. 3-49). Hellsdale, NJ: Lawrence Erlbaum Associates.

Hartley. A. A. (1993). Evidence for the selective preservation of spatial selective attention in old age. Psychology and Aging, 8(3), 371-379.

Hartley. A. A., Kieley, J. M., \& Slabach, E. H. (1990). Age differences and similaritics in the effects of cues and prompls. Joumal of Experimental Psychology: Hwhan Perception and Penformance, $16(3), 523-537$.

Hartley, A. A. \& Mckenzie, C. R. (1991). Altentional and perceplual contributions to the identification of extrafoweal stimuli: Adult age comparisons, Jourals of Geronology. psychological Sciences, 46(5), 202206.

Hasher, L., Zacks, R. T. (1988). Working memory, comprehension, and aging: Review and a new view. In $G$. H. Bower (Ed.). The Psychology of Ledming and Monivation, (Voll. 22, pp. 193-225). Orlando: Academic Press.

Hashtroudi, S., Chrosniak, L. D.. \& Schwartz, B. L. (1991). Effects of aging on priming and skill tearning Psychology and Aging, 6(4), 605-615.

Hebb, D. O (1949). The Orgranization of Behaviour. New York: John Wiley.

Holland, C. A., \& Rabbit, P. M. A. (1994). The problenis of being an older driver: Comparing the perceptions of an expert group and older drivers. Applied Ergonomics, 25(1), 17.27.

Houx, P. I., Jolles, J., \& Vreeling, F. W. (1993). Stroop interference: Aging affects assessad with the Stroop Colot-Word Test. Experinent Aging Research, 19(3), 209-224.

Hoyer, W. J., \& Familant: M. E. (1987). Adult age differences in the rate of processing expectancy information. Cognitive Dewelopment, 2(1), 59-70.

Huppert, F. A., Brayne, C. \& OConnor, D. W. (1994). Denenta and Normal Aging. New York: Cambridge University Press.

James, W. (1890). Priciples of Psychology. (Vol. 1). New York: Holt \& Co.

Jemings, I. M., \& Jacoby. L. L. (1993). Automatic versus intentional uses of memory. Aging, attention, and contral. Psychology and Aging, 8(2), 283-293.

Jetison, H. I. (1977). Vigillance. Biology. psychology, theory, and prachec. In R. R. Mackie (Ed.), Vigilance: 7heory, Operitional Performance and Phintological Correlates, (pp. 27 40). New York: Plenum Press.

Jolles, 1. (1986). Cognitive, emotional and behavhoral dysfunctions in aging and dementia. In D. F. Swata. E Fliers, M. Mirmitan, W. A van Gool, \& E. V. Hatren (Eds.). Progress in Brain Research, (pp. 15-39). Amsterdam: Elsevier Science Publishers.

Jonidas, J., Nayeh Benjamin, M. \& Palner, J. (1985). Assessing automaticity. Special Issue: Action, attention and automaticity. Acta Psychologica, 60(2-3), 157-171.

Jordan T. G., Rabbitt, P. M. (1977). Response times to stimuli of increasing complexity as a tunction of ageing. British Jomnal of Psychology, 68(2), 189-201.

Kahnemam, D. (1973). Attention and Effort. Englewood Clitis, NJ: Prentice Hall.

Karayanidis, F., Andrews, S., Ward, P. B. \& Michie, P. T. (1995). ERP indices of auditory selective attention in aging and Parkinson's disease. Psychophyrology, 32(4), 335-350.

Kaustar, D. H. (1982). Enperimental Psychology and Agng. New York: Holan Willey.

Kimchla, R. A (1980). The measurement of attention. In R. S. Nickerson (Ed.), Attention and Perfomance, (Vol. VIIL, pp. 213-238). Hillsdale, NI: Lawrence Erlbatm Associates.

Klein, R. M. (1994). Perceptual-motor expectancies interact with covert visual orienting under conditions of endogenous but not exogenous control. Special lssue: Shifts of visual attention. Camalian Joumal of
Experimenal Psychology, $8(2), 167-181$.

Kolb, B. \& Whishaw, 1. Q. (1990). Fundamentals of Human Nennopsychology. New York: Freeman, 


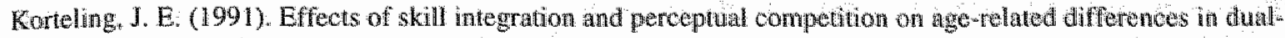
task performance Human Factors, $33(1), 3544$

Korteling, J E. (1993). Effects of age and lask sinthrity on dualask perfomance. Hwhom factors, $350(1), 90$ 113.

La Rue, A. (1992). Aging and Newropsychologicat Assersment. New Yonk Plenum Pros.

Leahey, T. H. (1980). A History of Psychology. Englewood Clins, NJ: Prertice Hall.

Leibniz, G. W. (1765). New Essays Concerning Human Understandang. Tramslated by W. Lamgley, 1916. London: Open Court.

Lorsbach, "T.C., \& Simpson, C, B. (1988). Dual-task performance as a function of adult age and task complexity. Psychology and Aging, 3(2), 210-212.

Mackie, R. R. (1987). Vigilance research-Are we ready for countermeasures Human Factors, 29,6), 707.724.

Madden, D. J. (1983). Aging and distraction by highly familiar stimuli during visual search. Dewelchomental Psychology, 19(4), 499-507.

Madden, D. J. (1985). Adult age differences im memory adriven selective attention. Developmental Psychowogy, $21(4), 655-665$.

Madden, D. J. (1986). Adult age differences in the attentional capacity demands of visual search. Cognitive Development, $I(4), 335-363$.

Madden, D. J. (1987). Aging, attention, and the use of meaning during visual search. Cogntine Development. $2(3), 201-216$

Madden, D. J. (1990a). Adult age differences in attentional selectivity and capacity. Special Issue: Cognitive gerontology. European Journal of Cognhtive Psychology, 2(3), 229-252.

Maddem, D. J. (1990b). Adult age differences in the time course of visual autention. Joumals of Geronology: Psychological Sciences, 45(1), 9-16.

Madden, D. J. (1992). Selective attention and visual search: Revision of an allocation model and application to age differences. Jommal of Experimental Psychalogy: Human Perception and Performance, 18(3), 821-836.

Madden, D. J. Connelly, S. L., \& Pierce, T. W. (1994). Adult age differences in shifting focused attention. Psychology and Aging. 9(4), 528-538.

Martin, D. R., \& Cranford, J. L. (1991). Age-related changes in binaural processing: II. Behavioral findings. American Journal of Otology, 12(5), 365-369.

Mathews, G., Davies, D. R. \& Holley, P. J. (1990). Extrawersion, wrousal and visual sustained attention: "The role of resource availability. Personality and Halidual Differences, 11(11), 1159.1173.

McCalley, L. T., Bouwhuis, D. G., Juola, J. F. (1995). Age changes in the distribution of visual attention. Joumals of Gerontology: Psychological Schences and Sacial Sciences, 50(6), 316.

McDowd, J. M. (1986). The effects of age and extended practice on divided attention performance. Journat of Gerontology, 41(6), 764-769.

McDowd, J. M., \& Biren, J. Ex (1990). Aging and attentional processes. In J. E. Birren \& K. W. Schaie (Eds.), Handbook of the Psychology of Aging, (3rd ed., pp. 222.233). San Dicgo, CA: Academic Press.

McDowd. J. M. \& Craik, F. I. M. (1988). Wffects of agng and task difficulty on divided atcention performunce. Journal of Experimental Psychology: Moman Perception and Performanco, $14(2), 267-280$.

Mckemna, F. P., Duncan, J., B Brown, I. D. (1986). Cognitive abilities and salety on the road: A treexamination of individual differences in dichotic listening and search for onbedded figures. Ergonomics, 29(5), $649-663$

Mesulam, M. M. (1990). Large-scale neurocognitive networks and distributed processing for attention, language, and memory. Annals of Neurology, 28(5), 597-613.

Mesulam, M. M. (1994). The multiplicity of neglect phenomena. Special issue: Spatial neglect. Position papers on theory and practice. Neuropsychological Rehabititation, 4(2), 173-176.

Molander, B., \& Backman, L. (1989). Age differences in theart rate patterns during concentratioub in a precision sport: Implications for attentional functioning Joumal of Gerontology: Psychological Sctences, 44(3), 80-87.

Moran, J., Desimone, R. (1985). Selectwe attention gates visual processing in the extrastriate cortex. Setence, $229(4715), 782-784$.

Morecraft, R. i., Geula, C.., \& Mesulam. M. M. (1993). Architecture of connectivity within a cingulowrontoparietal neurocognitiwe network for directed attention. Archive of Meurofrgy. $50(3), 279.284$.

Mountcastle, V. B. (1986). The neural mechanisms of cognitive functions can now be studied directly. Trends in Newrosciences, $9(10), 505-508$.

Mountcastle, V. B., Lynch, J. C., Georgopoulos, A., Sakata, H., \& Acuna, C. (1975). Posterior partetal association contex of the monkey: Command functions for operations within extrapersonal space. Joumal of Neurophysiology, 38(4), 871-908. 
Myerson, J; Halle, S., Wagstaf, D., Paon, L. W, 2: Smith; G. A. (1990). The informationoloss model: A

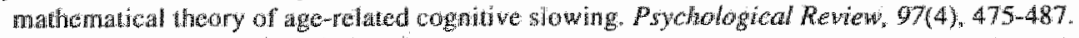

Nebeg, R. D. B Boller, $\mathrm{L}$. (1987); The use of latguage structure by demented patients in a visual searets task. Corter, 23(1), 87.98.

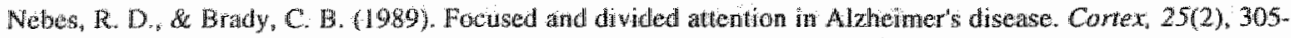
315.

Nobes, R. D, Madden, D, J (1983). The use of focused attention in wisual search by young and old adults. Expertwenal Aging Restarin $9(3), 139-143$.

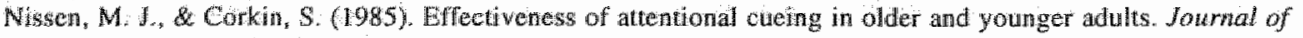
Gerontolog, $40(2), 185-191$.

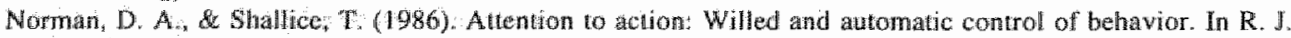

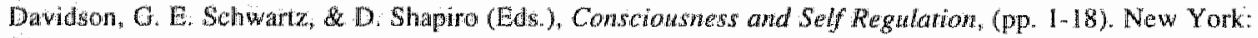
Plenum Press.

Panck, P. E. \& R maintain and reorient atditory selective attention. Experimemal Aging Rewearch, $7(4), 405.416$.

Panek, P. E., Rush, M. C, \& Slade, L. A. (1984). Locus of the age Stroop interference relationship. Jommal of Genetic Psychology. $145(2), 209.216$.

Parasuraman, $\mathbb{R}_{.}$\& Giambra, L. (199)\%. Skill dewelopment in vigilance: Effects of event rate and age. Prychology and Aging, 6(2), $155 \mathrm{~m} 169$.

Parasuraman, R. Nestor, P. C., Gronwood, P. (1989), Sustained attemtion capacity in young and older adults. Psychology and Aging. 4(3), 339-345.

Parasuraman, R, \& Nestor, P. G. (1991). Attention and driving skills in aging and Alaheinter disease. Special Issue: Safety and mobility of elderly driver: Part I. Human Factors, 33(5), 539.557.

Parasuraman, R., Wam, J.S. Dember, W. N. (1987). Vigilance: Taxonomy and utility. In L. Mark, J. S. Warm, \& R. L. Huston (Eds.), Ergonomics and Huwan Factors. Recent Research (pp. 11-32). New York: Springer-Verlag.

Pardo, J. V., Pardo, $\mathrm{P}_{*}$ Janer, $\mathrm{K}$. \& Raichle, M. E. (1990), The anterior cingulate cortex mediates processing selestion in the Stroop antention conflict paradigm. Proceedings of the Nariomal Academy of Science USA. $87(1), 256-259$

Park, D. C., Smith, A. D. Dudley, W. N., \& Lafroma, V. N. (1989). Erfects of age and a diwided attention task presented during encoding and retricval on memory. Jownal of Experimental Psychology: Leaming, Mewom. and Cogmition, $15(6), 1185-1191$. Pashler, H. (1994). Dual wask intererence in simple tasks: Data and theory. Psychological Bullen, 115(2), 220
244.

Plude, D. J., \& Doussard Roosevelt, J. A. (1989). Aging, selective attention, and feature integration. Psychology and Aging, 4(1), 98-105.

Plude, D.J., \& Hoyer, W. J. (1985). Attention and performance: Identifying and locializing age deficits. In $\mathbb{N}$. Chamess (Ed.). Aging and Howaw Performance; (pp. 47-99). Chichester, UK, John Wiley.

Ponds, R. W. Brouwer, W. H., \& Van Wolffelaar, P. C. (1988). Age differences in divided atcontion in a simulated aring task: Jowntals of Gerontology: Psychological sciences, 43(6), 151-156.

Paster, M. I. (1978). Chronometic Explorations of Mind. Hillsdale, NJ: Lawrence Eribaum Associates.

Posner, M. 1. (1980). Orienting of attention. Qutarterly Jounal of Experintental Psychology, 32(1), 3-25.

Posner, M. 1. (1988). Stutures and functions of selective attention. In T. Boll \& B. Bryant (E), Masfer Lectures in Chwical Nemopsychology. (Pp. 171-202). Washington, DC Anerican Psychological
Assobiation.

Posner, M. 1. (1995). Altontion in cogntive neuroscience: An overview. In M. S. Gazinaga (Ed.), The Cognitive Newmasciences: (pp. 615.624), Cambridge. MA: MTT Press.

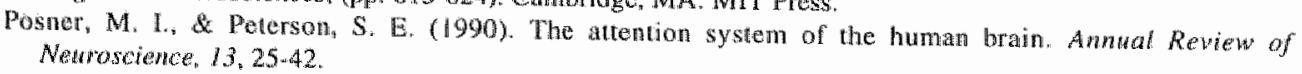

Posner, M. I. \& Raichle, M. F. (1994) Images of Mind. New York: Scientific American Library. Quilter, R. E, Giambra, L. M. (1983). Longitudinal age changes in vigilance over an eighteen year interval.
Jomwal of Gerontology, $38(1), 51-54$.

Rabbite. P. (1964). Set and age in a choicemresponse task. Joumal of Geronology, 19, $301-305$. Rabbit, P. (1982). Visual Seanch. In C. R. Pulf (Ed.), Hondbok of Reseanch Methods w Human Menory and
Cognirion, (pp. 27-62). New York: Academic. Press.

Rabbit, P., Vyns, S. M. (1980). Selective anticipation for events in old age. Joumal of Gerontology, 35(6),
913-919. 


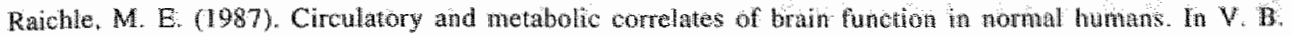

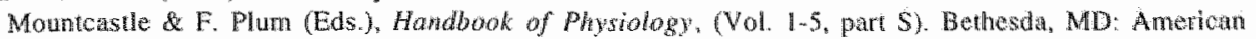
Physiological Socicy.

Raichle, M. E. Fiez, J. A., Videen, T. O. Macleod, A. M. K., Pardo, J W. Fox, P. T. E Petursen, S. E. (1994). Practice-related changes in human brain functonal anatomy durng nommotor leatring. Conthrol Cortex, $4(1), 8-26$

Reese. H. W., \& Rodeheaver, D. (1985). Prothlem solving and decision making. In I. E. Burran $\mathrm{K}$. W. Schate (Eds). Fandbook of we Psychology of Aging. (2nd ad. pp. 474-499). New York: Van Nostrand Roinhold.

Rogers, W. A. Bertus, E. L., \& Gibert, D. K. (1994). Dual-task assessment of age differences in atomatic process development. Psychology and Aging, 9(3), 398-413.

Rogers, W. A. \& Fisk. A. D. (1991). Are age differences in consistent-mapping visual search due to benure: learning or altention training? Psychology ond Aging, 6(4), 542,550 .

Rumelhart, D. E, \& MeClelland, J. L. (1986). Parallet distributed provesing. Explorations in the Microntucture of Cogntion. (Vol. 1). Cambridge, MA: Bradford.

Rush, M. C., Panek, P. E., \& Russeli, J. E. (1987). Cauhiousness and visual selective attention performance among older adults Jowrnal of Genetic Psychology, 148(2), 225-235.

Rush, M. C. Panck, P. E., \& Russell, J. E. (1990). Analysis of individual variability anong older adilts on the Stroop Color Word Interference Test. Imtemational Jownal of Aging and Human Development, 30(3), 225. 236.

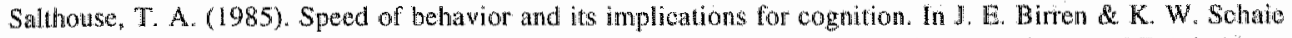
(Eds.), Handboak of the Psychology of Aging. (2nd ed., pp. 400-426). Now York: Vann Nostrand Reinhold.

Salthouse, "T. A. (1994). The aging of working menory. Special Section: Working menory. Neuropsychology, $8(4), 535-543$.

Salthouse, T. A. Fristow, N. M., Lineweaver, T. T., Coon, V. E. (1995). Aging of attention: Does the ability to divide deoline? Memory \& Cognition, 23(1), 59-71.

Salthouse, T. A., \& Pril, K. A. (1987). Inferences about age inpairnents in inferential reasoning. Prychology and Agmg, 2(1), 43-5I.

Salthouse, T. A., Rogan, J. D., \& Prill, K. A: (1984). Division of attention: Age differences on a visually presented memory task. Memory and Cognition, 12(6), $613 \ldots 20$.

Schneider, W., \& Slviffrin, R. M. (1977). Controlled and atutomatic human information processing; L, Detection, search and attention. Psychological Review $84(1), 1.66$.

Shallice, T. (1982). Specific impaiments of planning. In D. E. Broadbent L. Weiskranlz (Eds.), The Neuropsychology of Cagnitive Function, (pp. 199-209). London: The Royal Society.

Shallice, T. (1988). From Neuropsychology to Mental Stncture. New York: Cambridge University Press.

Shallice, T." \& Burgess. P. W. (1991a). Deficits in strategy applications following frontal lobe damage in man. Brana, 14012$), 727-741$.

Shallice, T. \& Burgess, P. W. (1991b). Higher-onder cognitive impanments and frontal lobe lesions in man. In

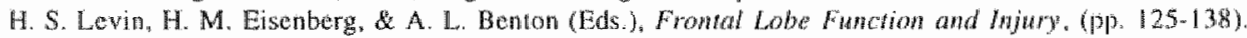
New York: Oxford University Press.

Shifrin , R. M., \& Schneider, W. (1977). Controlled and athomatic human information processing. II: Penceptakil learning. automatic attending and a general theory. Pyychological Reviz $w^{\prime \prime} 84(2), 127.190$.

Skinner, J. E., \& Yugling, C. (1977). Central gating mechatisms that regulate cwentwelated potentials and behavior. Progress in Clinical Newrophysology. $7,30-69$.

Smith, 5. (1983). Ideas of the Great Psychologists. New York: Haper \& Row.

Sokolov, E. N. (1963). Higher nervous funtions: The orienting rellex. Anmual Revitew of Physiology, 2.5.545. 580 .

Somberg. B. L., \& Sallouse, T. A. (1982). Divided attention abititics in young and old aduls, Journal of Experimental Psychology: Haman Percepron and Perfontance, 8(5), 651-663.

Stankov, L. (1988). Aging, attention, and intelligence. Pychology and Aging, 3(1), 59-74.

Stelmach, G. E., Amrhein, P. C., \& Goggin, N. L. (1988). Age differences in bimanual coordination, Jownats

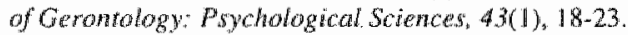

Stemberg, S. (1969). Memory-scanning: Mental processes revealed by reaction-ime experimonts Americass. Scientist, $57(4), 421-457$.

Strayer, D. L.. \& Kramer, A.F. (1994). Aging and skill acquisition: Learning performance distinctions. Psychology and Aging, $9(4), 589-605$.

Stroop, J. R. (1935). Studies of interference in serial verbal reactions. Joumal of Experimental Psychology, Is, $643-662$. 
Sirwillo, W. W. \& Quitur, R. E. (1964). Vigilance, age, and response-time. American Johmal of Psychology. $77,614-620$.

Surwillo, W. W., \& Quilter, R. E. (1965a). The infuence of age on latency tine of inwoluntary (galvanic skin reflex) and voluntary thes. Jotwnal of Gerontology, 20,173-176.

Surwillo, W. W. \& Quilter, R. E ( $1965 \mathrm{~b}$ ). The rellation of frequency of spontaneous skin potential responses to vigilance and age $P$ sychophysology, $1,272-276$.

Taub, H. A. (1973). Memory span, practice, and aging. Journal of Gerontology, 28(3), 335-338.

Thomas, J. C, Waugh, N, $C_{*}$ \& Fozatd, J, L. (1978). Age and familiatity in memory scannung. Journal of Geromtology。 33(4), 528:533.

Tichenter, E. B. (1908). Lectires on the Elennentary Psychology of Fetling and Attention. New York: Macmillan.

Treisman, A. M., \& Gelade, G. (1980), A feature-integration of attention. Cognitive Psychology. 12(1), 97-136.

Tun; P. A. \& Wingfield, A. (1995). Does dividing attention become harder with age? Findings from the Divided Attention Questionnaire. Aging and Cogninion, 2(1), 39-66.

Tun, P. A.. Wingfield, A, \& Stine, E. A. L. (1991). Speech-processing capacity in young and older aduits: A dual-task study. Psychology and Aging, 6(1), 3-9.

Tun, P. A. Wingfield, A. Stine, E. A. L., \& Meesas, C. (1992). Rapid speech processing and divided attention: Processing tate versws processing resources an an explanation of age effects. Psychology and Aging, 7(4), $546-550$.

Ulatowglka, H. K. Hayashi, M. M, Cannito, M. P., Fleming, S. G. (1986). Disruption of reference in aging. Brain and Language, 28(1), 24-41.

Van der Heijden, A. H. C. (1992). Selective Attention in Vision. London: Routledge:

Van Zomeren, A. H., \& Brouwer, W. H. (1994). Clinical Neuropsychology of Atrention. New York: Oxford University Press.

Vogt, B. A., Finch, D. M. \& Olson, C. R. (1992). Functional heterogeneity in cingulate cortex: The anterior executive and posterior evaluative regions. Special Issue: Cingulate cortex Cerebral Cortex, 2(6), 435-443.

Ward, L. M. (1994). Supramodal and modality-specific mechanisms for stimulus-driven shifts of auditory and visual attention. Special Issue: Shifts of viswal attention. Canadian Joumal of Experimentat Psychology. $48(2), 242-259$.

Wickens, C. D., Braune, R., \& Stokes, A. (1987). Age differences in the speed and capacity of information processing: 1. A dual-task approach. Psychology and Aging, 2(1), 70-78.

Woods, D. L. (1992). Auditory selective antention in middle-aged and elderly subjects: An event-related brain potential study. Electroencephalography and Clinical Neurophysiology. Evoked Potentials, 84(5), 456-468. 


\section{Chapter 4}

\section{Mild Traumatic Brain Injury, Attention,}

\section{and Aging}

"The fact that the various parts of the encephalon, though anatomically distinct, are yet so intimately combined and related as to form a complex whole, make it natural to suppose that lesions of greater or lesser extent in any one part should produce such general perturbation of the functions of the organ as a whole as to render it at least bighly difficult to trace any uncomplicated connection between the symptoms produced and the lesion as such."

- Ferrier 1886 -

\subsection{Introduction}

Each year, there are 2 million new patients with traumatic brain injury (TBI) in the United States alone, of whom almost $90 \%$ are classified as suffering from "mild" TBI (Goldstein, 1990; Miller, 1993). The true incidence of mild TBI is probably higher, because $20-40 \%$ of patients with mild TBI never seek medical attention (Frankowski, Annegers, \& Whitman, 1985). The incidence of traumatic brain injury is 200-300 per 100,000 in Scotland and the U.S. (Kraus et al., 1984, Miller, 1993), and the incidence of mild TBI is 131 per 100,000 per year (Kraus \& Nourjah, 1989). The causes of mild TBI are identical to those of moderate or severe TBI, with two exceptions: assaults and cervical acceleration/deceleration injury (CAD) are more common causes of mild than of severe TBI (Kraus \& Nourjah, 1988). Age-specific rates for persons with concussion show a peak occurrence from age 15-24, predominantly accounted for by males involved in motor vehicle crashes, and a decline until age 45 , whereafter an increase is seen through ages 65 and older, mainly caused by falls (Kraus \& Nourjah, 1989). The incidence of head injury in The Netherlands - compared to Scotland and the U.S. - is significantlly lower: about 107 per 100,000 (Centraal Bureau voor de Statistiek, 1993). Of all 


\section{CHAPTER 4}

patients hospitalized for head injury, 70-85\% were diagnosed as having sustained mild TBI, according to Clasgow Coma Scale estimates (GCS; Teasdale \& Jennett, 1974).

The number of road deaths has been falling for some years in many developed counties (Table 4.1). This is believed to be manly due to preventive measures such as seat belts, motorcycle helmets enforcement of laws on alcohol limits for drivers, speed limits, and improved car and road design (Department of Transport, 1995). The decrease in fatal accidents has consequently led to an increase in the proportion of mild and moderate head injuries. This means that more people will be dependent on rehabilitation services, since the kind of injuries that people sustain tend to change as a result of preventive measures. Galasko and colleagues (1993), for instance, showed a progressive increase in the number of patients seen in aecident and emergency deparments with a neck sprain after road traffic accidents since 1982, when the obligatory wse of seatbelts was introduced in Great Britain. Haslett, Duvall Young, and McGalliard (1994) even considered seatbelt use to be a najor factor contributing to the increase in CAD injuries, which might be greatly reduced by the use of air bags (Jagger, Vernberg, \& Jane, 1987).

Although older subjects may form a minority of people affected by TBI, they still pose a great burden on society, since they require more care in terms of length of hospitalization and in terms of support for persisting cognitive deficits (Kraus \& Nourjah, 1989). One of the most important changes in developed counties over the last 50 years has been the rapid increase in the number of people living beyond the age of 70 or 80 . Today, the average life expectancy in The Netherlands is about 77 years compared to 43 years in 1900 (Centraal Bureau voor de Statistiek, 1990). This increased longevity is seen in all people, including people who have sustained a TBI at some point in their lives. Because of this increase in life expectancy, we can also expect an increase in the duration of disability and dependence associated with TBI, which means that elderly people will consume a larger proportion of healthcare resources in the near future.

Table 4,1

Road Deaths per 100,000 Population

1980

1985

20

18

19

16

10

11

9
1993

17

16

11

8

7

A person who has sustained a head injury earlier in life may be more vulnerable to neuropathological aging, such as is the case in Alzheimer's disease (AD). In fact, moderate-tosevere head injury - among others - has been recognized as a potential risk factor for AD (Mortimer, 1994), although the potential risk of mild TBI remains elusive.

This chapter discusses the plausibility of the hypothesis that even mild TBI may have an influence on the usual process of cognitive aging. As we have learned more about the neurobehavioral consequences of severe head injury, we have also leamed to appreciate the less severe but similar consequences of mild TBI. There is a difference in degree, obviously, but not 
a difference in kind It is hypothesized that brain injury and aging compromise the bran's reserve capacity, and a model is described that might account for both the effects of cognitive aging and the effects of head injury. Special emphasis will be on the effect of TBI on attentional functions, since these functions appear to be especially affected by cognitive aging and also appear to be compromised after head injury.

\subsection{Milld Traumatic Brain Injury}

\subsubsection{Definitions}

By convention, a TBI is considered to be "mild" if the following characteristics are met (Ewans, 1992a; Levin, Eisenberg, \& Benton, 1989): brief loss of consciousness (LOC $<5$ minutes), a short period of post-traumatic ammesia (PTA $<24$ hours), an initial GCS score of 13-15, no evidence of focal injury on neurological examination, and nomal findings on traditional neurodiagnostic tests (i.e., EEG, CT, MRI, skull radiographs). TBI is considered to be "moderate" or "severe" when the GCS score is $9-12$ or 3-8, respectively. The classification of TBI in terms of severity, however, seems to depend heavily on the outcome criteria (e.g., extent of functional impairment, time spent in hospital, lacerations of the face and scalp, etc.). The Head Injury Interdisciplinary Special Interest Group of the American Congress of Rehabilitation Medicine, however, developed a useful working definition of mild TBI (see Esselman \& Uomoto, 1995). This definition is based on the various definitions of mild TBI that have been used since the influential article of Rimel, Giordani, Barth, Ball, and Jane (1981) and is summarized in Table 4.2.

Trable 4.2

Definition of Mild Traumatic Brain Injury

A patient with mild TBL has had a tramatically induced physiological disnuption of brain function, as manifested by at least one of the following:

1. Any period of loss of conseiousness

2. Any loss of memory for avens mmediately before or afien the accident

3. Any alternation in mental state at the time of the accident (e.g., feeling dazed, disoriented or confused)

4. Focal reurologicall deficit(s) may or may not be transient but where the severiny of the injury does not exceed the following:

a. Loss of consciousness of approximately 30 minutes or less

b. After 30 minutes, an initial Glasgow Coma Scale of 131015

c. Postmatamatic ammasia not greater than 24 hours

Mild TBI can occur without direct head strike (Binder, 1986), as - by definition- is the case in CAD injury (Yarnell \& Rossie, 1988). CAD injury is considered to be a form of mild TBI in that it is characterized by a different accident mechanism, and will therefore be referted to as CAD injury. CAD injury is defined as a trauma causing cervical musculoligament sprain or strain due to acceleration and/or deceleration of the head relative to the trunk in any plane (Hirsch, Hirsch, Hiramoto, \& Weiss, 1988). Injury to the cervical spine can take place in the sagittal plane by extension, flexion, or translation; in the frontal plane by lateral inclination or 
translation; or in the axial plane by rotation (Jeffreys, McSweeney, \& Park, 1980; Mac Nab, 1982). Because of the mechanics of bran injury in accidents involving acceleration/deceleration forces, certain brain injury profiles are common, including orbitofrontal, anterior and inferior temporal injury, and diffuse axonal injury (McAllister, 1992). The latter particularly affects the corpus callosum, superior cerebellar peduncle, basal ganglia, and periventricular white matter. In mild TBI axonal injury is usually generated by the transmission of shear forces through axial structures by angular acceleration (Adams, Graham, Murray \& Scott, 1982; Eisenberg \& Levin, 1989; Levin et al., 1987 ; Yokota, Kurokawa, Otsuka, Kobayashi, \& Nakazawa, 1991).

\section{2 .2}

\section{Cognitive Symptoms and Rate of Recovery}

Mild TBI is associated with two problems: an acute encephalopathy that lasts no more than a few days and a postconcussional syndrome (PCS) that may last for weeks, months, or even longer. The acute symptoms of mild TBI are nausea, vomiting, blurred vision, and somnolence. They are almost invariably short-lived and require no special treatment, according to Rutherford (1989). Persistence of acute symptoms beyond a few days is not characteristic of mild TBI.

Cognitive deficits after mild TBI may include poorer memory performance (Arcia \& Gualtieri, 1993; Gronwall \& Wrightson, 1981; Ruff et al., 1989) and slower response times (Jakobsen, Baadsgaatd, Thomsen, \& Henriksen, 1987; MacFlyn, Montgomery, Fenton, \& Rutherford, 1984), although the effects of mild TBI on memory are usually subtle (Gentilini et al., 1985; Hugenholtz, Stuss, Stethem, \& Richard, 1988). Mild TBI, however, primarily seems to affect attention (Gronwall, 1989; Parasuraman, Mutter, \& Molloy, 1991) and the efficiency of information processing (Beers, 1992; Hugenholtz et al., 1988; McMillan \& Glucksman, 1987). These cognitive deficits are most consistenlly seen when tasks are more complex, more demanding, or when they require controlled processing as opposed to automatic processing (see Schneider \& Shiffrin, 1977; Shiffrin \& Schneider, 1977). Although transient problems of mild TBI may arise, such as stuttering and difficulties in word retrieval, Jetter reversal, neading, or spelling, discrete cortical deficits (e.g., aphasia, agnosia, apraxia) are unlikely (Levin et al.,
1989).

Three months after the initial injury, most patients with mild TBI show substantial neurologic recovery, at least as assessed with commony used neuropsychological measures (Gentilini et al., 1985; Hugenholtz et al., 1988; Levin et al., 1987b). An even smaller proportion takes 3 to 6 more months to improve and recover completely (MacFlynn et al., 1984), although 10m $20 \%$ suffer some symptoms up to 6 months or Jonger (Alves, Colohan, OLLeary, \& Rimel, 1986 ;
Bolnnen, Twijnstra, \& Jolles, 1993; Bohnen, Twijnstra, Wijnen, \& Jolles, 1991; Bohnen,
Twijnstra, Wijnen, \& Jolles, 1992; Maton \& Twijnstra, Wijnen, \& Jolles, 1992; Mahon \& Elger, 1989; Middelboe, Birket Smith, Andersen, (1992b), and Bis, 1992). From other studies, Bohnen et al. (1994), Bohnen, Jolles, and Twijnstra, (1992b), and Bohnen, Jolles, Twijnstra, Mellink, and Wijnen (1995) derived evidence that up to $30 \%$ of patients with mild TBI may still show some symptoms after 6 months. Although patients show periodic impaiments when under physiological or tients with mild TBI, some MeCarthy, Gronwall Wrights when under physiological or psychological stress (Ewing, efficiency under suct 1989; Stuss et al., 1985). After 1 year 10 to $15 \%$ of patients with mild TBI have not recovered 
and the pattem of potential recovery in these subjects is langely unknown (McLean, Temkin, Dikmen, \& Wyler, 1983; Middelboe, Andersen, Birkit-Smith, \& Fris, 1992; Rutherford; Merrett, \& McDonald, 1979). It should be noted that the trajectory of recowery can change into a decline at any point after injury. There is evidence to suggest that new postconcussional symptoms can develop at any time during the first year after mild TBI (Alves et al., 1986). Some postconcussional symptoms may persist well beyond 3 to 5 years (Bohnen et al., 1994; Bohnen et al., 1995; Edna \& Cappelen, 1987), or even 30 years (Klein, Houx, \& Jolles, 1996), thus invalidating the traditional view that mild-to-moderate head injury involves an essentially reversible physiological process, as stated by Levin and colleagues (1987b).

\section{2 .3}

The Postconcussional Syndrome

The Postconcussional Syndrome (PCS) is a cluster of symptoms that frequently accur after mild TBI. The symptoms of PCS can be divided into three categories. Cognitive symptoms include subtle memory problems (79\% in the study by Rimel et al., 1981), poor concentration, distractibility, slowed thinking, sensitivity to light or noise, sleep problems, language impairments, impaired abstraction, and/or impaired judgment. Physical or somatic conplaints include headache, dizziness, fatigue, blurred viston, problems hearing, ataxia, neck pain, and/or disorders of smell/taste. Emotionall or affective complaints include depression, irritability, decreased drive, anxiety, emotional lability, impatience, and/or a poor frustration tolerance (Evans, 1992b). The mechanism by which injury affects various brain regions may give rise to distinct neuropsychiatric sequelae in a minotity of patients with mild TBL. Prominent impulsivity, affective instability, and disinhibition are seen infrequently and are secondary to injury to frontal, temporal, and limbic areas. Higher than expected rates of certain psychopathological disorders occur in the TBI population, such as psychotic syndromes and depressive syndromes (McAllister, 1992).

The mixture of diffuse and focal injuries, and the combination of cognitive, somatic, and behavioral difficulties do not fit easily into current diagnostic categories. Bohnen and Jolles (1992) and Brown; Fann, and Grant (1994) argue that there is sufficient empirical evidence to indicate that postconcussional symptoms occur and that they tend to have a predictable configuration. Brown and coworkers (1994) suggested that posiconcussional symptoms should therefore be recognized as a clinical entity and proposed the "Postconcussional Disorder" to be incorporated in the DSM-IV classification (American Psychiatric Association, 1994). Cicerone and Kalmar (1995), Henry (1994), and Hoffman (1994), however, suggested that the characterization of a single postconcussional syndrome may be misleading, and that it may be more meaningful to define a number of postconcussional syadromes with different symptom profiles and recovery.

Postconcussional symptoms may arise hours, days, or even weeks after the mild TBI. The

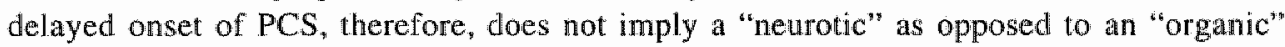
etiology (Rimel et al., 1981). The simultaneous occurrence of physical and emotional trauma means that conventional models of the PCS tend to be dichotomous, with symptoms being viewed as of physiological or psychological etiology. This division of opinion is most polarized after mild TBI. Lishman (1988) suggested that physiogenic as well as psychogenic influences are important in symptom genesis. However, where mild-to-moderate TBI is concerned, organic factors are mainly relevant in the earlier stages, whereas persisting symptoms are perpetuated by secondary neurotic developments, often of a complex nature (Lishman, 1988). 
More recent research suggests that the distinction between physiogenic as opposed to psychogenic etiology may no longer be useful (Bohnen \& Jolles, 1992). Psychosocial and cognitive behavioral factors may play a role in the onset of PCS, but more often are involved in the course and severity of PCS, allhough there is a large inter-individual wariability (Fenton, McClelland, Montgomery, MacFlynn, \& Rutherford, 1993; McClelland, Fenton, \& Rutherford, 1994; Montgomery, Fenton, McClelland, MacFlynn, \& Rutherford, 1991). Montgomery and coworkers (1991) furthermore suggested that organic factors, whilst important in the genesis of postconcussional symptoms, may also influence their persistence. Physiological and psychological factors also play a role in the genesis of PCS in CAD injury, but its time course appears to be rather different. In contrast to most cases of mild TBI, PCS symptoms in CAD injury do not disappear after 6 months, but tend to persist in a significant proportion of patients for more than I year (Ebbs, Beckley, Hammonds, \& Teasdale, 1986), more than 22 months (Norris \& Watt, 1983), or more than 2 years (Mac Nab, 1982).

It has been widely assumed that patients who sustain mild TBI or PCS develop a post-tranmatic stress disorder (PTSD) in response to their cognitive difficulties, diminished coping skills, or other losses. The PTSD diagnosis refers to emotional symptoms of an unusually distressing event (American Psychiatric Association, 1994). Sbordone and Liter (1995), however, showed that none of their patients with mild TBI reported symptoms such as intrusive recollections of the tratumatic event, nightmares, hypervigilance, phobic or startle reactions, or became upset when they were asked to describe the traumatic event or were exposed to stimuli associated with it. Their data suggest that PTSD and mild TBI are two mutually exclusive disorders, and that it is highly unlikely that patients with mild TBI develop PTSD symptoms.

Psychophysiological and cognitive processes mediate the effects of stress on the patient at all stages (Steptoe, 1991). In this sense, brain damage itself must be considered as a distinctive form of stress since the very organ of coping has been damaged, i.e, an impaired ability to maintain vocational, domestic or academic responsibilities. Current multifactorial concepts of chronic pain, which emphasize the integration of biomedical with cognitive, affective, and behavioral factors, may prove to be a helpful model in explaiming postconcussional symptoms (Turk \& Rudy, 1991). It is only through the recognition and identification of separate processes mediating the effects of stress that cquestions about outcome, the limits of the impact of organic and psychosocial factors, the nature of exaggeration or malingering, and appropriate therapy can be resolved (Jacobson, 1995).

4.2 .4

Variables Affection Recovery

Outcome varies with the nature of the injury, the support received by the victim, the promptness and efficiency of rehabilitation, the passage of time, and on the victim's mental capacity including functions such as problem-solving style, concentration, planning, and behavioral monitoring. The mental efficiency system can collectively be referred to as the "executive functions", presumably residing in the frontal lobes, which are compromised by TBI (e.g., Lewin \& Kraus, 1994). As a rule, the depth of coma is predictive of outcome; so is the duration of coma, and so is the extent of brain tissue destruction. In fact, these three elements are interrelated. The fact that the outcome of an injury is related to the severity of the injury may seem like a truism, but is actually a good example of the Rule of Severity, a clinical corollary to Lashley's Mass Action Law (see Kolb \& Whishaw, 1990). Since patients with mild TBI tend to have less extensive brain damage than patients with moderate or severe TBI their prognosis is 
relatively good. However, some factors not related to the injury itself might affect outcome. Potential risk factors for persisting postconcussional symptoms after mild TBI include a lower educational level, alcohol abuse (Evans, 1992b), and female sex (Rutherford et al., 1979). A study of patients with CAD injury (Radanov, Di Stefano, Schnidrig, \& Ballinari, 1991) identified pain severity at outset (but not premorbid psychological state) as a predictor of the development of lingering sequelae in mild TBI. Whille young men have a higher risk of sustaining a mild TBI, older women are more at risk of developing chronic sequelae than are young men (Fenton et al., 1993). A lower socioeconomic status is also predictive of a prolonged period of recovery (Rimel et al., 1981).

The trajectory of recovery may be enhanced by certain factors like, for example, a higher level of intelligence. A high premorbid IQ is one of the strongest predictors of good outcome (Gianotta, Weiner, \& Karnaze, 1987) and correlates positively with the percentage of postinjury employment (Mayes, Pelco, \& Campbell, 1989). If the job demands, however, are high relative to preinjury performance, then even a minor loss can lead to an inability to meet job demands and hence to unemployment (Adamovich, Henderson, \& Auerbach, 1985). Multiple mild TBIs lead to slower recovery of information processing speed than is observed following a single mild TBI, suggesting that cumulative cognitive impairment may also follow multiple episodes of mild TBI (Binder, 1986; Evans, 1992b; Gronwall, 1989; Gronwall \& Wrightson, 1975). As will be reviewed in the next paragraph, attentional deficits are one of the most prevalent cognitive deficits after mild TBI. Recovery of atientional functions may be extremely important in the process of rehabilitation, since attentional capacity is a prerequisite for almost every other cognitive function.

\subsection{Mild Traumatic Brain Injury and Attention}

\subsubsection{Mild Traumatic Brain Injury and Attentional Capacity}

Deficits of attention are among the most common symptoms following TBI (Binder, 1986; McKinlay, Brooks, Bond, Martinage, \& Marshall, 1981; Oddy, Coughlan, Tyerman, \& Jenkins, 1985; Van Zomeren \& Van den Burg, 1985). The severity of the neuropsychological deficits has been found to be related to TBI severity, including duration of PTA (Wrightson \& Gronwall, 1981), coma duration (Dacey et al., 1991; Dikmen, McLean, \& Temkin, 1986; Dikmen, Temkin, McLean, Wyler, \& Machamer, 1987; Dikmen, Machamer, Temkin, \& McLean, 1990; Rimel et al., 1981), coma depth (Alexandre, Colombo, Nertempi, \& Benedetti, 1983; Dikmen et al., 1986; Levin et al., 1990), pupil reactivity (Levin et al., 1990), and extensiveness of lesions (Alexandre et al., 1983; Gennarelli et al., 1982).

Longitudinal studies suggest that, although attentional deficits decrease over time, they may linger for several years after severe TBI (for review, see Gronwall, 1987). Mild TBI in general, however, is not associated with long-term persisting neuropsychological impairments (Dikmen et al., 1986; Dikmen, Machamer, Winn, \& Temkin, 1995; Gentilini et al., 1985; Levin et al., 1987b; McLean et al., 1983). Previous follow-up studies investigating the time course of recovery of attentional processes after mild TBI have produced conflicting results. Early studies using neuropsychological batteries (Barth et al., 1983; O'Shaughnessy, Fowler, \& Reid "1984; Rimel et al., 1981) and response time measures (Van Zomeren \& Deelman, 1976; Van Zomeren \& Deelman, 1978) reported persistent attentional deficits 3-12 months post-injury. These 
studies had methodological problems including a lack of appropriate control groups and use of inadequate selection criteria. Thus subjects with substance abuse and/or moderate injuries (PTA, I week) were included. Several better controlled studies have demonstrated recovery of attentional processes to a level comparable to that of matched control groups within 3 to 6 months post-injury (Gentilini, Nichelli, \& Schoenhuber, 1989, Gronwall \& Wrightson, 1974; Gronwall \& Wrightson, 1980; Levin et al., 1987b; MacFlynn et al, 1984; McLean et al., 1983). Other studies suggest longer trajectories of recovery (Bohnen et al., 1993; Bohnen et al., 1991; Bohnen et al., 1992b). Gronwall (1991), however, cautioned that return of test scores to normal does not necessarily imply full recovery from head injury. For example, nonSymptomatic students, who had a mild concussion 1 to 3 years earlier showed impaired scores. on a vigilance task only when they were tested under conditions of mild hypoxia (Ewing et al., 1980). In addition, subtle attentional deficits may become apparent in demanding or complex tasks. Hugenholtz and coworkers (1988) used complex choice response time tasks designed to assess selective attention and central processing. Their results suggested that the return to normal functioning may not be complete 3 months after mild TBI. Consistent with this, Gentilini and colleagues (1989) found that selective and divided attention were still impaired in subjects with mild TBI 3 months post-injury. Further evidence for persisting effects of mild TBI was obtained by comparing concussed individuals with their uninjured monozygotic twins. The concussed twins had an inferior performance in speed and divided attention tasks when tested at least 3 years after they sustained their injury (Dencker, 1958).

Not all aspects of attention appear to be equally impaired, however, and results are not always consistent, even for the same aspect of attention (Van Zomeren \& Brouwer, 1987). There is limited information about the nature and magnitude of attentional impaiment as a function of severity of head injury. Dikmen and Levin (1993) identified several methodological problems leading to inconsistent results, such as differences in the classification of TBI severity, biases in selection of patients with mild TBI, and use of controls to account for comorbidities related to other system injuries, pre-existing conditions, and emotional reactions to and circumstances surrounding the accident. One of the biggest disadvantages of most studies, however, is the fact that they tended to restrict their experimental group to either mild, moderate, or severe cases (Dikmen et al., 1986; Dikmen et al., 1990; Levin et al., 1990; Levin et al., 1987b). In 1995 Dikmen ar. 1 colleagues (1995) reported on the neuropsychological outcome at 1 year postinjury of patients with TBI ranging in severity from mild to severe. They found the magnitude and pervasiveness of the neuropsychological impairments to be dependent on the severity of the head injury. Impairments of attention started to emerge as the severity of TBI increased. Dikmen and colleagues showed that even when a single index of severity is used (e.g." duration of coma or PTA), there is essentally a continuum of recovery, with small differences in severity being reflected in small differences in outcome."

As will be demonstrated in the paragraph on the pathophysiology of TBI and as shown by Dikmen and colleagues (1995), the kind of neuropsychological deficits after mild TBI tend to differ from those of moderate and severe TBI more in terms of quantity rather than in terms of quality. Therefore, the next paragraphs discuss the effect of mild and moderate or severe TBI on most of the attentional domains mentioned in chapter 3 . When the prefix "mild" is omitted the studies refer to moderate or severe TBIs, or the experimental group consists of patients with mild, moderate, and severe TBI. When available, descriptions will be provided concerning the effects of mild TBL on attentional capacity. 
According to Posner (1988), selective attention involves the selection of higher levels of processing, including conscious processing, while preventing the access of other signals to those same high levels of processing. In a visual response time selective attention task, Van Zomeren (1981) found that patients with severe TBI needed significantly more time to overcome the interference from distraction. Stuss et al. (1989) compared three groups of TBI patients for their ability to ignore redundant information during a choice response time task (i.e., chronic severe TBI, and mild TBI assessed at various intervals; and severe TBI tested within 7 to 10 months after injury). Findings indicated that selective attention capacity is primarily compromised in chronic severe TBI, since these patients were not able to ignore the redundant information. The patients with mild and severe short-term TBI, however, did not appear to be affected in a negative way. In a study with a group mainly consisting of patients with mild TBI, Dencker (1958) simultaneously presented spoken texts and a tape recording of a group of people talking, and also found no evidence for an increase in interference susceptibility. Their subjects, however, were tested on average 10 years after injury, by which time most of the patients with mild TBI will have recovered. Gronwall and Sampson (1974) confirmed their conclusion by using a dichotic listening test with patients with mild TBI within 24 hours of admission to the hospital. Spoken texts were presented to both ears, while subjects were to follow the words presented to the left ear and ignore the words presented to the right. Once again, patients with mild TBI and controls did not differ in the amount of interference from the to be ignored ear. In a simulated functional task involving comprehension of a spoken passage, Kewman, Yanus, and Kirsch (1988) showed that the performance of patients with moderate-to-severe TBI was inferior to that of controls especially under conditions of competing vocal stimuli: Subjects with TBI reported having significantly more difficulty comprehending the target passages in the presence of the interfering passages than did the control subjects.

A cued response time task, using the covert orienting paradigm of Posner, Nissen, and Odgen (1978), was used to measure the response time benefit of valid directional cuing and the response time cost of miscuing in two groups of Australian Rules football players who had sustained mild TBI (Cremona-Meteyard \& Geffen, 1.994). The first group was tested within 2 weeks post-injury and showed the same cost as healthy controls in speed of response to targets in the unexpected visual field. However, their responses to targets in the expected location (following valid cues) showed only a minor benefit compared to controls. When these subjects were retested 1 year later, their pattern of performance had not altered but overall response time had improved. These findings were replicated in the second group tested at least 1 year after mild TBI. Moderate-to-severely injured patients also showed a normal cost but a reduced or absent benefit, which according to Cremona-Meteyard, Clark, Wright, and Geffen (1992) may underlie some problems in higher order executive functioning (see Norman \& Shallice, 1986). An alternative explanation could be that the patients with TBI had difficulty interpreting a cue as a signal to orient attention.

A typical task to measure selective attention in the clinical setting is the Stroop Color Word Test (SCWT; Stroop, 1935). The SCWT is a perceptual interference task and examines the speed at which color names and / or colors are read, and the speed at which color names are read when the printing ink color is different from the color name. The latter aspect is usually referred to as the interference effect, since subjects have to suppress their reading response in favor of naming 
the collor in which the words are printed. Ponsford and Kinsella (1992) and Van Zomeren and Brouwer (1987) found no evidence for the presence of a specific interference susceptibility in patients with severe TBI, but rather suggest a deficit in the general speed of information processing: Collor naming and word reading scores on the SCWT were among the neuropsychological measures shown to be the best measures of this deficit (Ponsford \& Kinsella, 1992). Stuss and coworkers (1985) also showed an overall slower reading speed in their TBI patients relative to the control group. Howewer, both groups were similarly affected by the interference. Previously, MeLean and coworkers (1983) showed the severity of TBI to be related to the amount of interference on the SCWT. Testing at 3 days post-injury indicated that patients with mild TBI had significant difficulty in preventing interference, but not in reading. Testing after 1 month showed that the patients with less severe TBI - according to the length of PTA - performed at the same level as their matched controls. Test results 6 months post-injury showed no differences between TBI patients of either severity and controls. Bohnen and colleagues (1992b), however, showed that patients with mild TBI with postconcussional symptoms 6 months after injury had a higher interference susceptibility than patients with mild TBI without postconcussional symptoms and matched controls, leaving open the possibility that McLean and colleagues (1983) investigated symptom-free TBI patients. Bohnen, Jolles, and Twijnstra (1992a) and Bohnen, Twinnstra, and Jolles (1992) showed that a modified, more dernanding SCWT could better differentiate between subjects with or without persistent symptoms at 3 months post-injury and that subjects with postconcussional symptoms may have more difficulty with perceptually interfering stimuli than patients with mild TBI without persistent postconcussional symptoms. Consistent with Bohnen and colleagues (1992b), Wilberger (as cited in Wilberger, 1993) showed that American football players who had postconcussional symptoms (headache, dizziness, and difficulty with concentration) 3 months post-injury performed significantly worse than symptom-free football players. Previously, however, Leininger, Gramling, Farrell, Krentzer, and Peck (1990) stated that the severity of neuropsychological deficits in patients with mild TBI with postoncussive symptoms is independent of neurological status immediately following injury. Dikmen and colleagues (1995) studied - among others-SCWT performance at 1 year post-injury in a group of 436 patients with mild to severe TBI. They found that patients as a group performed significantly worse than the tratuma controls. This effect was shown only by patients who had an altered consciousness for at least 7 days, suggesting that interference suseeptibility is no longer present 1 year after mild TPI.

In another mixed TBI group, Vakil, Weisz, Jedwab, Grosswasser, and Aberbuch (1995) studied the probable underlyng mechanisms of selective attentional deficits such as excitation, inhibition, and habituation. Control and TBI subjects were compared on four conditions based on the SCWT (neutral, habituation, Stroop, and negative priming). TBI patients did not show a slower reading time on the negative priming as compared to the Stroop interference condition, which led the authors to conelude that TBI is characterized mainly by an impairment of inhibitory mechanisms.

Cognitive disturbances of patients with mild TBI may be subtle and only demonstrable with very sensitive or modified tests. Bohnen and colleagues (1992a), for instance, modified the SCWT by adding an interference subtask of increased complexity. This subtask required more flexibility for the naming and reading of the items depending on a special visual cue given with the words. Bohnen and colleagues (1992a) found patients with mild TBI to be significantly slower than controls on this modified subtask, whereas there was no significant difference 
between patients with mild TBI and controls with the original interference subtask. Bolnen and colleagues (1992) furthermore showed that the modified SCWT could better differentiate between subgroups of patients with mild TBI with or without persisting postconcussional symptoms than the original SCWT. From this they concluded that patients with postconcussional symptoms may have more difficulties with perceptually interfering stimuli than patients without persistent postconcussional symptoms. These findings are in acoord with the results of Stuss and coworkers (1989), who reported that TBI patients performed more poorly than did control subjects (abeit, inconsistently so) on a computerized measure of selective attention. Batchelor, Harvey, and Bryant (1995) investigated the sensitivity of a modified SCWT similar to the one Bohnen and coworkers (1992a) used to index attentional deficits in patients with mild TBI, and confirmed Bohnen and coworkers' findings, but furthermore found that patients with mild TBI also performed more poorly than controls on the original and interference conditions of the SCWT. Finally, Batchelor and coworkers (1995) remarked that the modified SCWT (Bohnen et al., 1992a) appears to be a sensitive measure of selective attention once the mediating effects of anxiety are taken into account.

Another way to measure the effect of response interference is by using visual choice response times. Van Zomeren and Brouwer (1987) used a four-choice response time task with a panel containing distractor lights in close proximity to the target lights. Their patients with moderateto-severe TBI, who were tested between 3 and 6 months post-injury, had longer response times in the standard condition without interfering lights than did controls and were disproportionally more affected by the presence of irrelevant stimuli next to the target lights. The heightened distractibility after moderate-to-severe TBI expressed by longer response times does not appear to wane easily, as demonstrated by Deland, Vanier, Lambert, and Provost (1992), who used the same task as Van Zomeren and Brouwer (1987). They presented the same task at 3 and 12 months post-injury to a group of consecutive patients hospitalized for TBI and found that the improvement for both the standard task and the distraction task was roughly the same. MacFlynn and coworkers (1984) administered a similar four-choice response time test to subjects with mild TBI 24 hours, 6 weeks, and 6 months post-injury. These subjects displayed a significantly poorer performance than the matched controls at 24 hours and at 6 weeks. They, however, also showed an improvement up to 6 months post--jnjury, whereafter their scores excelled those of matched controls.

In conclusion, interference susceptibility appears in the early stages of recovery but disappears in most subjects with mild TBI after 3106 months. The lack of effect in earlier studies might be due to the fact that the cognitive sequelae of mild TBI tend to be subtle, and likewise require more sensitive neurocognitive tests to demonstrate any interference effect at all. Another explanation might be the fact that the distinction between targets and distractors in some tasks is too obvious to evoke conflicting response tendencies. Tests that serve to measure strong response tendencies; and thus lend themselves to an evaluation of possible "focused attention deficits" (Shiffrin \& Schneider, 1977), have mostly been used, but usually also yielded negative results. Patients with mild TBI in the subacute stage, however, appear to have an increased interference susceptibility, as was demonstrated with a modified version of the SCWT. Another relevant factor in relation to interference susceptibility, or the lack of inhibitory control, is the wisual and acoustic hyperesthesia which is frequently found in patients with mild TBI (e.g., Bohner, Twijnstra, Kroeze, \& Jolles, 1991; Bohnen et al., 1991; Waddell \& Gronwall, 1984) and CAD injury (Norris \& Wat, 1983; Radanov et al., 1991: Radanov, Dvorak, \& Valach, 1992; Radanov, Sturzenegger, De Stefano, \& Schnidrig, 1994; Radanov* 
Sturzenegger, Di Stefano, 1995). A decreased tolerance to light and sound may be a manifestation of the lack of inhibitory control by orbital frontal cortex areas over sensory information processed by posterior brain areas and subcortical centers. These frontal areas are particularly vumerable to injury because they lie against the base of the skull (Walsh, 1987).

\section{3 .3}

\section{Mild Traumatic Brain Injury and Divided Attention}

Divided attention refers to the perfomance of two or more simultaneous tasks and thus requires parallel processing of several imputs (McDowd \& Birren, 1990). The ability to divide attention between two concurrent tasks is a valid test procedure to detect deficits in processing resources. The very lew studies explicitly taking into account divided attention in TBI patients used quite heterogeneous patient selection criteria, making interpretation of the results particularly difficult. Even so, divided attention seems to be affected the most after TBR.

Gentilini and coworkers (1989) administered a divided attention task to a group of patients with mild TBI I month after injury. The task consisted of a simple response time task to stimuli appearing in the lateral visual fields combined with the request to count backward, by $2 \mathrm{~s}$ from 100 to 0 at a regular pace. One month after injury the patients with mild TBI were significantly slower than controls. However, the differences between TBI patients and controls had disappeared 3 months post-injury in a sample of 22 of their initial 48 patients with mild TBI.

Several authors have suggested that choice response time tasks are generally more effective than simple response time tasks in showing residual impairment after TBI (e.g., Ponsford \& Kinsella, 1992; Stuss et al. 1989). By using a visual choice response time task with increasing stimulus alternatives in patients with severe TBT, Miller (1970) showed these patients to be slower than controls. Furthermore, their patients with TBI appeared to be affected more by an increase in task complexity. In a sample of patients with mild TBI tested within 24 hours, Gronwall and Sampson (1974) showed that mild TBI was associated with a decrease in performance only in the most complex subtests of symbolic and non-symbolic $2,4,6,8$, and 10-choice tasks. In accordance with Miller"s results (1970) for severe TBI, they judged the central processing time to be also affected in mild TBI. In general, choice response time studies show that TBT appears to be associated with a deficit in controlled information processing (Shiffrin \& Schneider, 1977), thus leading to longer response time when the number of stimulus allematives increases or when the stimulus-response compatibility decreases (for an extensive review of choice response time experiments in patients with moderate-to-severe TBI, see Van Zomeren \& Brouwer, 1994).

The Paced Auditory Serial Addition Test (PASAT; Gronwall \& Wrightson; 1974; Gronwall, 1977) is usually taken as an index of the information processing rate and strongly depends on the ability to divide attention between several processes. In many follow-up studies the PASAT has been used as a measure of neurobehavioral recovery. The PASAT involves dividing attention between stimuli, stored memory elements, mental transformations, and responding. The subject is presented with a series of single digits and instructed to add them in pairs, so that the first number is added to the second, the second to the third, and so on, and to give his or her answer aloud. In the original PASAT, four lists of 61 digits are presented with an interstimulus interval of $2.4,2.0,1.6$, and 1.2 seconds. The percentage of correct responses is usually taken as a measure of performance. Gronwall and Wrightson (1974) found that rapid presentation of digits had a significant influence on the performance of patients with mild TBI and concluded that lower PASAT performance under time pressure is probably the result of slow execution of 
a quaritatively normal control strategy (see Shiffrin \& Schneider, 1977). Subjects were tested several times over a period ranging from 24 hours to 70 days after injury, and it was demonstrated that PASAT scores were initially reduced in TBI subjects with and without postconcussional symptoms. These scores improved over time, and the improvement on PASAT performance was associated with a reduction in postconcussional symptoms (Gronwall, 1976). An indication that processing speed is the critical factor is conveyed by the 1977 study of Thomas (as cited in Van Zomeren \& Brouwer, 1994), who found no differences in the processing speed of patients with mild TBI when digits were presented at a lower rate. A remarkably fast recovery of function on the PASAT was seen in American fooball players who sustained a mild TBI. PASAT scores returned to normal within 10 days after injury (Barth et al., 1989).

Newcombe, Rabbitt, and Briggs (1994) examined patients with mild TBI within 48 hours of their accident and 1 month later, using modified versions of the PASAT, but found no significant differences between TBI patients and controls at either time. The inability to replicatie the findings of Gronwall and Sampson (1974) might be attributed to differences in interstimulus intervals, selection of patients and controls, and the total length of testing in the Newcombe and colleagues (1994) study, which also included several other neurocognitive tests.

The reduction in performance on the PASAT does not appear to be caused only by a generalized slowing in patients with mild TBI, as Barth et al. (1989), Cremona-Meteyard and Geffen (1994), and Hugenholtz et al. (1988) found no initial differences between patients and controls on simple response time measures. Patients with mild TBI who suffered postconcussional symptoms for longer than 1 month had slower response times within 5 days after injury than patients whose symptoms resolved (Jakobsen et al, 1987). The study of Leininger and colleagues (1990), in which a modified wersion of the PASAT was used (interstimulus interval $=2.8,2.4,2.0$, and 1.6 seconds; \# digits: 26 ), showed that the total number of correct solutions over 4 trials was lower in patients with mild TBI referred for neuropsychological evaluation between $\mathbb{1}$ and 22 months post-injury compared to that of matched controls. Patients with mild TBI who lost consciousness during injury had test scores similar to those of patients who experienced disorientation or confusion but no loss of consciousness.

With regard to the possible mechanisms underlying divided attention performance in TBI, Stablum, Leonardi, Mazzoldi, Umilta, and Morra (1994) conducted a number of experiments in patients who sustained severe TBI several years before testing. In their first experiment, they used a dual-task paradigm (Umilta, Nicoletti, Simion, Tagliabue, \& Bagnara, 1992), in which the same stimulus required either 1 or 2 responses, depending on the experimental conditions. In their second experiment, they used the task shifting paradigm of Morra (cited in Stablum at al., 1994) in which different stimuli required different responses.

The first experiment showed differences in response time associated with the need for response coordination between the single task and double task to be greater for TBI subjects. Stablum and colleagues (1994) considered this to be indicative for a specific impairment in monitoring and control functions. Previously, Umilta and colleagues (1992) showed that experiment I demands coordination of responses. The fact that TBI subjects performed worse than controls suggests that the efficiency of high-level control processes is affected in a negative way. In terms of the Norman-Shallice model (Norman \& Shallice, 1986), the Supervisory Attentional System (SAS) appears to be compromised by TBI. This model is discussed in detail in paragraph 4.3.2. Further evidence for a SAS deficit was provided by the second experiment in which they found the cost of shifting from one task to the other to be greater in TBI subjects. 
This effect was only present in the condition in which short series of stmuli were presented and a new task progratin could thus be preactivated. The lack of effect in the longer series suggests that contention scheduling as such is not compromised by TBT:

\subsubsection{Mild Tramatic Brain Injury and Sustained Attention}

Sustained attertion performance involves the detection of infrequent and unpredictable events after prolonged periods of observation (Parasuraman, Wam, \& Dember, 1987) and has usually been assessed by target detection accuracy, response time to correct detections, false alarm rates, and sometimes by the decrease in vigilance over time. Overall, vigilance refers to the obserwer's ability to detect and respond to stimuli efficiently throughout a task, whereas a decrease in vigilance refers spectfically to a progressive decline in performance over time - the hallmark of a deficit in sustained attention (Parasuraman, 1984; Parasuraman et al., 1991). Studies assessing sustained attention have reported deficits in overall levels of vigilance performance in molerate to severe TBI (Levin, High, Goldstein, \& Willams, 1988; Wilkins, Shallice, MoCarthy, 1987 ) as well as in patients with mild TBI (Dikmen, McLean, Temkin, \& Wyler, 1986; Parasuraman et al, 1991). To test a possible decline in vigilance, Dikmen and colleagues (1986) used the Seashore Rhythm Test - a test for non-verbal auditory perception (see Reitan \& Wolfson, 1989). They demonstrated a decrease in vigilance at 1 month postinjury, but this decrease was resolved when subjects mild TBI were tested 1 year later. In fact, only subjects who took at least 14-28 days before they could follow oral commands still performed worse after 1 year. Dikmen and colleagues (1986) replicated this finding, showing that patients with mild TBI tend to perform a little less adequately than uninjured subjects. Bugler and Snyder (1995), however, found that mild TBI negatively affected Seashore Rhythm test performance in two of their four patients who sustained mild TBI 5 and 10 months previously. Despite neuropsychological findings that suggested significant sequelae by using Tscore transformations (see Lezak, 1983), quantitative post-injury MRI studies did not show differences in preinjury neuroimaging between patients with mild. TBI and control subjects. Their sample size was small. Dikmen et al. (1995) included 436 subjects in their study.

Gentilini and colleagues (1989) used a simple response time task to stimuli appearing in the lateral visual fields as a measure of sustained attention and found their 48 patients with mild TBI to be affected 1 month after injury. When retested 2 months later, no differences were found between controls and patients with mild TBI $(22$ of the initial 48 subjects with mild TBI tested at 1 month). The 1977 study by McCarthy (as cited in Gronwall, 1989) showed that subjects with mild TBI performed at the same level as old (>80 years) subjects on an auditory vigilance test. Both groups had an inferior performance when compared to that of a young control group, but when the subjects with mild TBI were tested 4 to 6 weeks later, they no longer differed from the young control group and both young groups performed significantly better than the 80 -year-old subjects.

Ewing and colleagues (1980) demonstrated that residual brain impairment may be present even after the resolution of cognitive impairment and symptoms. They used an auditory vigilance task in a group of patients who sustained mild TBI 11 to 3 years earlier and who had fully recovered (i.e., they showed no postconcussional symptoms). The mild TBI group and control group were tested at ground level and at a simulated altitude of 12,500 feet. The mild TBI group and control group performed similarly at ground level, but the mild TBI group performed significantly worse under conditions of mild hypoxia. 
The few studies that examined vigilance performance over time showed a decrease in vigilance in severe TBI (Levin et al., 1988; Loken, Thornton, Otto, \& Long, 1995), but not in mild TBI (Ewing et al., 1980; Parasuraman et al., 1991). The studies by Buchtel (1987), Parasuraman et al. (1991), and Van Zomeren and Brouwer (1987), however, also did not show a dechne in vigilance performance over time in TBI. Loken and colleagues (1995) contributed the lack of time-on-task effects in the Van Zomeren and Brouwer (1987) study to the fact that the more severely injured patients who were unable to reach the $75 \%$ detection level necessary to complete the task were excluded. The decrease in vigilance over time in the 1995 study of Loken and colleagues (1995) was not significantly related to the severity of TBI in terms of length of impaired consciousness. Therefore, it is questionable whether the impaiment of sustained attention over time in severe TBI reflects increased neural damage or other injury-related factors. Loken and colleagues (1995) furthermore showed that patients with severe TBI were not significantly affected by an increase in task complexity. Therefore, they did not consider the decrease in vigilance performance to be consistent with an attentional capacity model of impaired vigilance in TBI.

Another explanation, namely an inability to maintain adequate arousal, is also not supported by empirical evidence, although Gentilini and coworkers (1989) suggest that a general deficit in maintaining sustained attention might explain the fact that their patients with mild TBI were not particularly sensitive to concomitant tasks. Davies and Parasuraman (1982), however, demonstrated that a decrease in tonic arousal caused by heat or alcohol-induced fatigue did not result in a decrease in vigilance performance over time, although they did show a decrease in overall vigilance performance. Brotwer and Van Wolffelar (1985) showed that TBI patients even tended to have an elevated level of arousal when heart rate variability on EEG activity was used as an index of tonic arousal.

Parasuraman and coworkers (1991) suggested that a decrease in phasic arousal may be reflected by a lowered attentional performance in mild TBI. Phasic arousal is thought to occur when the ascending reticular activation system (ARAS) activates the cortex in response to a warning stimulus in the environment (see Mesulam, 1981; Scheibel, 1980). Disorders in phasic arousal have been suggested to result in a heightented susceptibility to non-relevant stimuli (Stuss \& Benson, 1984). Several studies indeed suggest that TBI is associated with a reduction in phasic arousal (Curry, 1980; Rugg et al, 1989). Parasuraman et al. (1991) showed that patients with mild TBI could not maintain adequate vigilanee levels unlless they received external feedback on how well they were doing. Supportive feedback, however, could not prevent a decreatse in vigilance over time in patients with severe TBI (Loken et al., 1995), thus questioning the plausibility of a phasic arousal decrement model to explain vigilance changes over time. An explanation that is supported by the study by Loken and coworkers (1995) is that TBI subjects appear to adopt a less efficient response strategy when confronted with "noisy" visual stimuli. In a four-choice response time task they found that TBI subjects sacrificed speed for increased accuracy, whereas they sacrificed accuracy for speed in an eight-choice response time task.

In summary, overall vigilance performance appears to be affected in patients who have sustained mild-to-severe TBI. In addition to overall impaired vigillance, attention in severe TBI also appears to be affected in a negative way when measures of time-on-task are evaluated. The underlying mechanisms responsible for the vigilance decline in these patients remain elusive. 


\subsection{Pathophysiology of Mild Traumatic Brain Injury}

TBI may give rise to diffuse, microscopic damage to the brain, especially the subcortical white matter (Ahara, Hall, Pitts, Fukuda, \& Noble, 1995; Bourke, 1988). Trauma physically distupts eells, and subsequent ischemia causes metabolic derangement. Further histopathological events cause an increased neuropsychological impairment in survivors. After focal injury, the center of the lesion is necrotic, and the surrounding margin of tissue is compromised by edema and inflammation (Gennarelli, 1993). In patients with focal injury, edena develops over the first few days after trauma, whereas patients with diffuse lesions usually exhibit edema on admission (Alexander, Martin, Khanna, Caron, \& Becker, 1994). The necrotic center becomes contracted and scarred. At the periphery of the lesion the pathological changes are largely reversible, so that much of the function returns (Towbin, 1987). The difference between mild, moderate, and severe TBI is based on the degree of diffuse and (mulli)focal damage.

Cortical injury is transmitted through axial structures, generating shear forces that produce scattered; multifocal subcortical and brain stem axonal damage (Blumbergs, Jones, \& North, 1989; Bourke, 1988). This "diffuse axonal injury" (DAl) has been found postmorten throughout the neuraxis in severe, moderate, and even in mild TBI (Oppenheimer, 1968), with the number of damaged neurons corresponding with the severity of TBI (Povlishock \& Coburn, 1989). DAI in mild TBI has also been demonstrated by Adams et al. (1982), by Eisenberg and Levin (1989), and by Yokota et al. (1991). Although subjects who sustained mild TBI may have axonal damage, Povlishock and Coburn (1989) suggested that the traumatically injured axon might be capable of some adlaptive form of recovery. The functional recovery after DAI strongly depends on the health of the largely unaffected surrounding tissue and its ability to compensate for tissue that is lost. Mittl and colleagues (1994) suggested that this DAI may represent the pathologic substrate underlying the PCS that occurs in a substantial proportion of patients with mild TBI. It should be noted, however, that although the magnitude of DAI is related to the forces released during the accident, this relation is not of a simple linear nature (Elson \& Ward, 1994).

Head injury can occur without direct head strike (Binder, 1986), as is the case in CAD injuries. (Croft, 1995; Yarnell \& Rossie, 1988). Although most experimental TBI research has been performed on animals (Jane, Steward, \& Gennarelli, 1985; Powlishock, Becker, Cheng, \& Vaughan, 1983), the neuropathologic and newrochemical changes in animals after TBI show a strong resemblance to those seen in humans (Clifton, Ziegler, \& Grossman, 1981; Gennarelli, Adams, \& Graham, 1986). Therefore it is reasonable to assume that mild TBI in experimental animals is a valid representation of what happens in humans. In primates, experimental CAD injury has been shown to cause both surface and intracerebral petechial hemorrhages (Ommaya, Fuas, \& Yarnell, 1968; Ommaya \& Gennarelli, 1974). The biomechanical forces released by the accident may cause damage especially to the limbic and frontotemporal cortices (Ommaya \& Gennarelli, 1974). The cerebral symptoms observed after CAD injury (i.e, deficits mainly in attention and concentration, to a lesser extent in memory, and least in higher cognitive functions) are compatible with damage to frontobasal cerebral structures and the upper brain stem (Kischka, Ettlin, Heim, \& Schmid, 1991).

The previousiy held assumption that traumatic brain damage evokes immediate and irreversible damage to the brain parenchyma and its intrinsic vasculature (Povlishock \& Coburn, 1989) has been questioned by Povlishock and Jenkins (1995). Ott, McClain, Gillespie, and Young (1994) 
and Povlishock and Jenkins (1995) suggested that traumatically induced axonal damage is not the immediate consequence of traumatic tissue tearing. Rather, they consider that it is a delayed consequence of complex axolemmal and/or cytoskeletal changes evoked by specific tissue damage to the brain which then lead to cytoskeletal collapse and impairment of axoplasmic transport, ultimately progressing to axonal swelling and disconnection.

The axonal tracts that are most likely to be disrupted in TBI are the monoaminergic projections from the brain stem to cortical and subcortical structures. The majority of monoaminergic neurons are located in the lower brain stem and project to the reticular formation and subsequently project to different target areas via long efferents (Azmitia, 1987). The location, size, and extensiveness of these connections make this network especially vulnerable to the forces associated with TBI (Gualtieri, 1995). TBI can result in increased levels of neurotransmitters, resulting in excessive stimulation of specific receptors and in chronic deficits in cholinergic neurotransmission. Axonal injury may contribute to central cholinergic neurotransmission defects wia diffuse deafferentation, which in part may explain the memoty deficits often seen after mild TBI (Dixon, Taft, \& Hayes, 1993; Hayes, Lyeth, \& Jenkins, 1989). TBI can affect forebrain cholinergic and catechs:aminergic innervation, which mediate: memory, concentration, and problem solving (Schmidt \& Grady, 1995). Varney and colleagues (1995) demonstrated regional cerebral blood flow (CBF) abnormalities in the anterior mestal temporal regions, whereas posterior temporal abnormalities were considered to be very infrequent in patients with mild TBI. Schmidt and Grady (1995) found evidence of a significant and apparently selective loss of ventrobasal forebrain cholinergic neurons following brief concussional injury in rats, a type of injury that is known to produce significant disturbance in cognitive tasks linked to neocortical and hippocampal cholinergic function. Diminished levels of the monoamine metabolites of dopamine and serotonin have been demonstrated by Gualtieri (1990), Rosner, Newsome, and Becker (1984), and Silver, Yodofsky, and Hales (1991). At the neurobehavioral -level, injury to monoamine systems generates changes in arousal, attention, and information processing, which are mediated in large by dopaminergic and noradrenergic neurons (Carlsson, 1987), and alterations in mood, appetite, sleep, sexual function, pain perception, circadian rhythms, motor activity, memory, anxiety, and aggression, which are mediated by serotonergic neurons (Meltzer \& Lowy, 1987).

\section{5 Mild Traumatic Brain Injury and Cognitive Aging}

Changes in cognitive performance associated with normal aging often overlap those accompanying the early, mild stages of AD (Rubin, Morris, Grant, \& Vendegna, 1989; Storandt \& Hill, 1989). As in normal aging, memory loss is the most prominent and earliest symptom of AD (Butters, Granholm, Salmon, Grant, \& Wolfe, 1987; Morris \& Kopelman, 1986; Nebes, 1989). However, attentional functions are also affected in the early stages of this disease (Baddeley, Logie, Bressi, Sala, \& Spinnler, 1986; Filoteo et al., 1992; Nebes \& Brady, 1989; Parasuraman, Greenwood, Haxby * Grady, 1992). In a review of the literature, Parasuraman and Haxby (1993) concluded that visual attention shifting and divided attention were the most prominently affected attentional functions in patients with mild AD. They suggested that attention represents the first cognitive indicator of neocortical dysfunction in 
early $\mathrm{AD}$. Disconnection between frontal and posterior parietal areas may mediate the selective disruption of attentional functions in $\mathrm{AD}$. In addition to the overlap in cognitive functioning between ustal aging and $\mathrm{AD}$, there is overlap in the pathological changes in some brain areas: Although amyloid plaques and neurofibrillary tangles are pathognomonic features of $\mathrm{AD}$, they also appear in the same areas in normal aged brains, albeit to a lesser degree (Matsuyama \& Nakamura, 1978, Mornison, 1993; Price, Davis, Mornis, W White, 1991). Normal aging differs from $A D$ in seweral ways synaptic length increases in the elderly but not in $A D$ (DeKosky \& Scheff, 1990), and neurofibrillary tangles are rare in the normal neocortex, although they do occur in subcortical areas (Mortson, 1993). However, in advanced age the similarities between age and $\mathrm{AD}$ may increase. The dendritic growth seen in the dentate gyrus in normal aging regresses in 86 to 95 year olds (Flood, Buell, Defiore, Horwitz, \& Coleman, 1985). The similarity between aging and $\mathrm{AD}$ in both functional loss and pathology has been noted, but different conclusions have been drawn about the relation between the two states. These conclusions mainly depend on whether $\mathrm{AD}$ is regarded as a disease distinct from the normal aging process, or whether $\mathrm{AD}$ is viewed as a part of the normal aging process (Huppert $\&$ Brayne, 1994). However, the current level of knowledge of aging and AD does not allow us to give definite answers. Consequently, knowing whether the effects of AD and aging on attention differ quantitatively and/or qualitatively is important for both theoretical and practical reasons.

Older patients with mild TBI appear to be at greater risk of prolonged disability (Binder, 1986; Rutherford et al., 1979; Wrightson \& Gronwall, 1981). Patients who sustained mild TBI when they were older than 40 years are twice as likely to develop persistent symptoms as patients under the age of 30 (Denker, 1944). A rather alarming neuropsychological follow-up study by Mazzucchi and colleagues (1992) showed mild deterioration in $10 \%$, moderate deterioration in $25.7 \%$, diffuse deterioration in $28.6 \%$, and even dementia in $21.4 \%$ of head-injured subjects aged over 50 years and tested at 6 to 36 months post-injury. These incidences did not substantially change when only patients with mild TBI (i.e., no loss of conscionsness, GCS 13-15, PTA $<24$ hours, and negative CT findings) were considered, implying that mild TBI could be followed by rather severe consequences. Similar, though less dramatic, results were obtained from studies by Houx, Vreeling, and Jolles (1991), and by Houx and Jolles (1994), who found that biological life events (BLEs), such as mild TBI, could accentuate the effect of "usual" biological aging in subjects who furthermore considered themselves to be normal and thealthy. In a multiple cohort study it was suggested that these BLEs might even give rise to predementing stages of AD (Jolles, Houx, Vreeling, Bothen, \& Reyersen van Buuren, 1990). From a controlled postal survey carried out 1 to 5 years after mild TBI, Bohnen and colleagues (1992b) concluded that TBI may produce long-term neurasthenic symptoms to a much higher degree than normally present in a person's everyday life.

4.5 .2 Mild Traumatic Brain Injury, Age and Alzheimer"s Disease: Pathophysiological
Findings

The etiology and pathogenesis of AD are largelly unknown. Although many risk factors have been proposed, the evidence for most of these is weak except for older age (Hagnell, Lanke, Rorsman, \& Ojesjo, 1981; Jorm, Korten, \& Henderson, 1987) and a family history with AD (Fatrar, O'Sullivan, Cupples, Growdon, \& Myers, 1989; Nee et al., 1983). The link between TBI and a subsequent neurodegenerative process is largely circumstantial, but some observations suggest a possible link between TBI and dementia. In the first place, TBI by itself 
can result in a post-traumatic encephalopathy dementia syondrome. If "TBI is severe enough to produce either retrograde or anterograde ammesia, permanent memory abnomalities of ten occur. Language is abnormal in a third of patients surviving moderate to severe TBI (Cummings \& Benson, 1992). TBI can furthermore give rise to personality, mood, and motor abnomalities, psychomotor retardation, and impairnent in abstraction, calculation, attention, and plaming, although the precise nature of these symptoms depends upon the site of damage. As might be expected from the most prevalent accident mechanism, the frontal and temporal lobes are most frequently affected (Absher \& Cummings, 1994).

Another observation suggesting a causal relationship between TBI and AD is that several case reports have described pathologically confirmed $\mathrm{AD}$ caused or accelerated by a single TBI episode (Corsellis \& Brierly, 1959; Rudelli, Strom, Welch, \& Ambler, 1982). These studies concluded that elderly people in particular appear to be susceptible to the effects of TBI and thus are at higher risk of neuropathological aging.

The perhaps strongest and most reported indication of the possible relation between TBI and AD is the dementia pugulistica syndrome, which, according to Corsellis, Bruton, and FreemanBrowne (1973), is characterized by neuropathologic abnomalities of the septum pellucidum, scarring of the cerebral and cerebellar cortices, cell loss in the substantia nigra, and accumulation of neurofibrillary tangles in the cerebral cortex and brain stem. Dementia pugilistica is the result of the cumulative clinical effects of repeated mild TBI and had already been recognized in boxers in 1928 and was referred to as the "punch-drunk syndrome" (Martland, 1928). Ever since, several other authors have pointed at the possibility that prior head injury and multiple trauma may lengthen the period of recovery (Carlsson, Svardsudd, \& Welin, 1987; Edna \& Cappelen, 1987). Roberts, Allsop, and Bruton (1990) demonstrated diffuse amyloid plaques in the neocortex of 14 of Corsellis' original patients (aged 22-83 years; Corsellis et al., 1973), and in 6 additional boxers. All their patients with dementia pugilistica with substantial neurofibrillary tangle formation showed an abundance of amyloid plaques. The extensiveness of the amyloid plaques was comparable to that seen in 20 confirmed cases of AD. Molecular markers in the amyloid plaques and neurofibrillary tangles of dementia pugulistica were the same as those in AD. Also, clinical symptoms, distribution of pathology, and neurochemical deficits were similar. Clinton, Ambler, and Roberts (1991) re-investigated a case of post-traumatic premature $\mathrm{AD}$, previously reported by Rudelli and colleagues (1982). Immunocytochemistry using antibodies to amyloid beta-protein revealed large numbers of "diffuse" non-Congophilic plaques with little or no neuritic component. A similar preponderance of this plaque type is present in the brains of boxers with dement ia pugilistica. Clinton and coworkers (1.991) judged their observations to be supportive of the idea of a trauma-induced $\mathrm{AD}$-like degenerative process and suggested that such a condition is associated with a marked preponderance of "diffuse" amyloid plaques. The mechanism of neurofibrillary" tangle formation induced by repetitive TBI may be different from that in AD, however: Creddes, Vowles, Robinson, and Sutcliffe (1996) reported on a case of a 23-year-old boxer whose brain showed neurofibrillary tangles in all neocortical areas, but not in the sites that are involved early in $\mathrm{AD}$ - namely, the entorhinal cortex and the hippocampal formation (Braak \& Braak, 1991). Neurofibrillary tangles are usually only found in the neocortex in the late stages of AD. To their surprise, Geddes and coworkers (1996) found no amyloid plaques, which -according to Roberts and colleagues (1990) - are a common feature of dementia pugulistica, in association with neurofibrillary tangles, although Hof and colleagues (1992) detected amyloid plaques only in 1 of the 3 patients with dementia pugulisticat that they studied. Beta amyloid deposition on its 
own has been seen after a single severe TBI (Roberts, Gentleman, Lynch, \& Graham, 1991; Roberts of al., 1994), but it is possible that it may also occur as a secondary phenomenon (Roses, 1994) rather than as a primary event. Neurofibrillary tangles may precede anyloid deposition, but amyloid deposition is not a prerequisite for the development of neurofibrillary tangles. Apart from the fast that the deposition of beta amyloid in the brain plays a key role an the pathogenesis of $A D$, apolipoprotein $\mathrm{E}$ epsilon 4 allele (APOE-4) is a strong risk factor for $\mathrm{AD}$, and there is in vitro evidence that $\mathrm{APOE}-4$ is directly involved in beta anyloid deposition (Mayeux et al., 1995). As will be reviewed in the next section, TBI is an epidemiological risk factor for $\mathrm{AD}$, and deposition of beta amyloid occurs in approximately one-third of individuals dying after a severe head injury. This might also be the case in mild TBI, as Blumbergs@ and colleagues (1994) showed multifocal axonal injury in patients with mild TBI with persistent symptoms and subtle neuropsychological deficits, using immunostaining with an antibody to the amyloid precursor protein, a marker of fast axonal transport. Nicoll, Roberts, and Graham (1995) reported that the frequency of APOE-4 in individuals with beta amyloid deposition following head injury is higher than is found in most patients with $A D$, while in TBI patients without beta amyloid deposition the APOE-4 frequency is similar to that of AD-free controls. Their finding provides evidence linking APOE-4 with beta amyloid deposition in vivo and suggests that known environmental and genetic risk factors for $\mathrm{AD}$ may act in a cumulative way. In line with this observation, Mayeux and colleagues (1995) suggested that the biological effects of head injury may increase the risk of $\mathrm{AD}$, but only through a synergistic relationship with APOE-4, They found a tenfold increase in the risk of AD in the presence of both APOE-4 and a history of traumatic head injury, compared with a twofold increase in risk with APOE-4 alone. Head injury in the absence of APOE-4 did not increase risk.

Mild Traumatic Brain Injury, Age and Alzheimer's Disease: Epidemiological Findings

Single epidemiological studies that focused on identifying previous TBI as a possible risk factor for AD have not yielded unanimous conclusions. Mortimer and colleagues (1983) initially demonstrated a significant association between previous TBI and AD, whereafter two other studies also reported a significant positive correlation (Graves et al., 1990; Heyman et al, 1984). In five other studies, TBI was more frequent in patients with AD than in controls, but the association between "TBI and AD could not be confirmed statistically (Amaducci et al., 1986; Broe et al., 1990; Chandra, Kokmen, Schoenberg, \& Beard, 1989; Chandra, Philipose, Bell, Lazaroff, \& Schoenberg, 1987; Shalat, Seltzer, Pidcock, \& Baker, 1987). In contrast with these findings, several other authors did not report any association at all (Bharucha, Schoenberg, \& Kokmen, 1983; Chandra, Kokmen, \& Schoenberg, 1987; Ferini-Strambi, Smirne, Garancini, Pinto, \& Franceschi, 1990; Soininen \& Heinonen, 1982; Sulkava, Erkinjuntt, \& Palo, 1985).

The EURODEM Risk Factors Research Group (Mortimer et al., 1991 ) performed a re-analysis of 11 case-control studies (marked in Table 4.3 with *) including TBI patients with loss of consciousness and restriction of comparisons to community (versus hospital) controls. A test for heterogeneity across studies was negative, and data could thus be pooled in subsequent analyses.

Although significant associations within individual datasets were absent, the pooled relative risk for head trauma of these studies -expressed by the Odds ratio- was 1.82 (95\% confidence 
interval $=1.26-2.67)$. Stratified analyses showed stronger associations in patients without a positive family history of dementia and in males (wersus females). Adjustment of the poolad relative risk for family history of dementia, sex, age of onset, education, and alcohol consumption did not alter significantly the association between head trauma and $\mathrm{AD}$. The authors found no interaction effect between head trauma and family history of dementia, suggesting that these risk factors operate independently. The findings of the pooled analysis support an association between reported head trauma and AD. The TBI patients in these studies, however, conld be classified as moderate-to-severe, thus questioning the plausibility of mild TBI as a risk factor for AD. Up till now, only one study has investigated mild TBI. In a recent study, Rasmusson, Brandt, Martin, and Folstein (1995) examined patients with mild TBL, particularly those without a genetic risk for $\mathrm{AD}$. Medical history including TBI in 68 patients with $\mathrm{AD}$ and 34 non-demented controls was gathered from their spouses. $\mathrm{AD}$ patients were categorized as familial, sporadic, or uninformative. Head injury was reported in 20 of the AD subjects and in 1 control. Twenty percent of the patients with familial AD and $43.5 \%$ of the patients with sporadic AD reportedly had a premorbid TBI. TBI had no effect on age of dementia onset. These results indicate that even mild TBI may be a predisposing factor to $\mathrm{AD}$. particularly in the absence of a clear genetic contribution.

Table 4.3

Traumatic Brain Injury as Risk Factor for Alzheiner"s Disease

\begin{tabular}{|c|c|c|c|c|c|c|}
\hline \multirow[t]{2}{*}{ Authors } & \multirow{2}{*}{$\underset{\#}{\text { AD Patients }}$} & \multicolumn{2}{|c|}{ Controls } & \multirow[t]{2}{*}{ Odds Ratio } & \multirow[t]{2}{*}{$\mathrm{S} / \mathrm{NS}$} & \multirow[t]{2}{*}{ EIORODEM } \\
\hline & & Type & 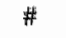 & & & \\
\hline Soininen \& Heinomen (1982) & 63 & $\mathrm{H} / \mathrm{C}$ & 91 & 0.6 & NS & * \\
\hline Heyman et al. (1984) & 40 & $\mathrm{C}$ & 80 & 5.3 & S & $*$ \\
\hline French et al. (1985) & 78 & $\mathrm{H} / \mathrm{C}$ & 78 & 4.5 & NS & \\
\hline Mortimer et al. (1985) & 78 & $\mathrm{H}$ & 76 & 4.5 & $S$ & * \\
\hline Amaducci et al. (1986) & 116 & $\mathrm{H}$ & 116 & 3.5 & NS & * \\
\hline Chandra et al. (1987) & 64 & $\mathbb{H}$ & 64 & 6.0 & NS & $*$ \\
\hline Shalat et al. (1987) & 98 & $\mathrm{C}$ & 162 & 2.4 & NS & $*$ \\
\hline Kokmen et al. (1988) & 392 & $\mathrm{C}$ & 392 & 1.4 & NS & * \\
\hline Chendra et al. $(1989)$ & 274 & $\mathrm{C}$ & 274 & 1.2 & NS & \\
\hline Holman ef al. (1989) & 198 & $\mathrm{C}$ & 198 & 1.3 & $\mathrm{NS}$ & * \\
\hline Broe et al (1990) & 170 & $\mathrm{C}$ & 170 & 1.3 & NS & $*$ \\
\hline Perini-Strambi et al. (1990) & 63 & $\mathrm{C}$ & 126 & 1.0 & $\mathbb{N S}$ & \\
\hline Graves et all ( $(1990)$ & 130 & $\mathrm{C}$ & 130 & 3.0 & $S$ & 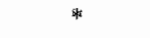 \\
\hline Kondo \& Y Yamashita (1990) & 34 & $\mathrm{C}$ & 68 & 18.0 & $\mathrm{NS}$ & * \\
\hline Paschalis et al. (1990) & 103 & $\mathrm{C}$ & 90 & 2.7 & $\mathrm{NS}$ & \\
\hline
\end{tabular}

$\mathrm{H}_{3}$ hospital controls

C. Community controls

$\mathrm{OR}$, Odds Ratio, which is an estimate of the relative risk, scores above 1.0 indicate elevated risk.

S, P $\leq 0.05, \mathrm{NS}, \mathrm{P}>0.05$.

* detaset included in EURODEM study (Mortimer et al., 1991).

In conclusion, several factors may explain the likelihood of an association between TBI and AD. In the first place, TBI may damage the blood-brain barrier (Mathew et al., 1994; Nawashiro, Shima, \& Chigasaki, 1994), resulting in loss of immunological protection of brain tissue and entry of toxins and/or viruses that could produce damage many years later (Mortimer et al, 1985; Mortimer \& Pirozzolo, 1985). Secondly, head trauma may damage the functioning 
of brain tissue to a degree that does not produce a detectable cognitive loss, but which reduces the functional brain resterwe capacity. Given that increasing age appears to be associated with a gradual decrease in brain reserve capacity, such a loss in brain reserve could shorten the time before the threshold for clincally diagnosable cognitive deterioration is reached. A detailed description of a brain reserve capacity model of cognitive decline in aging and TBI is provided in the next paragraph.

\section{A Model for Mild Traumatic Brain Injury as a Risk Factor for Accelerated Cognitive Aging}

\subsubsection{Brain Reserve Capacity}

The brain reserve capacity model is based on the assumption that cognitive aging is characterized by a discontinuty caused by some lesion in the central nervous system (CNS; Satz, 1993), and that brain reserwe capacity is affected by disease states and aging. Although neurons in the human brain are normally incapable of mitosis after early childhood, the abundance of neurons in the human brain may cause cerebral lesions to become clinically evident only after massive loss. In the case of Parkinson's disease, for instance $85 \%$ of the cells of the nigrostriatal system have to be depleted and a similar decrease in dopamine cells has to occur before clinically demonstratable parkinsonism is present (Quinn, Rossor, \& Marsden, 1986; Roth, 1986). Similar thresholds have been observed in multiple infarct dementia, where the total volume of separate lesions has to exceed a certain level before symptoms appear, (Roth, 1986), and in AD, where amyloud plaques and neurofibrillary tangles have to exceed a quantitative threshold before giving rise to clinical symptoms (Miller, Hicks, D'Amato, \& Landis, 1984; Tomlinson, Blessed, \& Roth, 1970).

With regarcl to TBI, Symonds (1962) noticed: "In concussion there may be rapid and complete recovery of cerebral function; but this does not necessarily exclude the possibility that a small number of neurons may have perished - a number so small as to be negligible at the time, but leaving the brain more susceptible as a whole to the effects of further damage of the same kind." Ewing and colleagues (1980) demonstrated that this is the case when the CNS is stressed by mild hypoxia: in non-symptomatic patients who were mildly concussed 1 to 3 years earlier had an imparred cognitive performance. Another example of a possible threshold is provided by the dementia pugulistica syndrome (Corsellis et al., 1973), where several single mild TBIs finally give rise to the development of AD-like symptoms. The dementia pugulistica syndrome shares several neuropathological features of AD: the accumulation of neurofibrillary tangles (Corsellis. et all., 1973) and amyloid plaques (Roberts et al., 1990).

"The mechanism that might ultimately lead to an accelerated cognitive decline with increasing age is depicted in Figure 4.1 and is based on two main principles: brain reserve capacity, which is related to the amount of remaining functional brain tissue, and a threshold concept, which corresponds to the critical anount of brain tissue at which normal cognitive function can no longer be sustained. Threshold phenomena are incompatible with simple continuum theories. These postulate that the effects of a given disorder merge insensibly with normal variation with respect to the associated symptoms and deficits and the causes underlying them. When there is a linear relationship between a disease and normal wariation, any significant correllations that are found between measures of behavior and associated biological variables should make it possible 
to predict with precision the features and biological concomitants at the extreme ends of the distribution. However, neither the cognitive behavioral and other chinical features nor the neuropathological characteristics of $\mathrm{AD}$ can be forecast in this mamer on the basis of data for normal elderly persons of comparable demographic characteristics. Aging is associated with an increased neuronal loss, which, for instance, is expressed by a reduced brain weight, a reduced. number of pyramidal neurons; , reduced degree of dendritic branching (Katzman et al., 1988), or a reduced cerebral perfusion (Stern, Alexander, Prohovnik, \& Mayeux, 1991). This reduction in functional brain mass represents the reduced brain reserve capacity in the model of Satz (1993). It must be stressed, however, that lesions which are associated with TBI tend to be quite distinct from lesions associated with aging. In the latter, the term lesian refers to the progressive loss of brain tissue with advancing age. TBI is characterized by the sudden onset of lesions caused by external factors. In Figure 4.1 , the brain reserve capacity is represented by the length of the bar. The hypothetical subjects \#1 and \#2 sustained a single TBI, whereas subject \#3 sustained multiple TBIs. The reduced brain reserve capacity associated with aging may cause latent symptoms to become manifest when a certain threshold is exceeded due to the capacity reducing effect of $\mathrm{TBI}_{\text {, }}$ as is the case in subject \#2. In subject \#3, the functional impairment is only expressed after multiple TBIs as, for instance, is the case in dementia pugulistica. The first subject sustained the same TBI as subject $\# 2$, but his or her clinieal symptoms remain subthreshold. The reason for the lack of clinical symptoms in this subject is supposedly due to the beneficial effects of protective factors that increase the brain reserve capacity. Subject \#3 showed no clinical symptoms after a single TBI, because of the same protective factor that benefitted subject \#\#. The second TBI, howewer, was sufficient to aggravate latent previously subthreshold symptoms, which could no longer be compensated anymore by the brain reserve capacity

\section{Figure 4.1}

Brain Reserve Capacity (Satz, 1993)

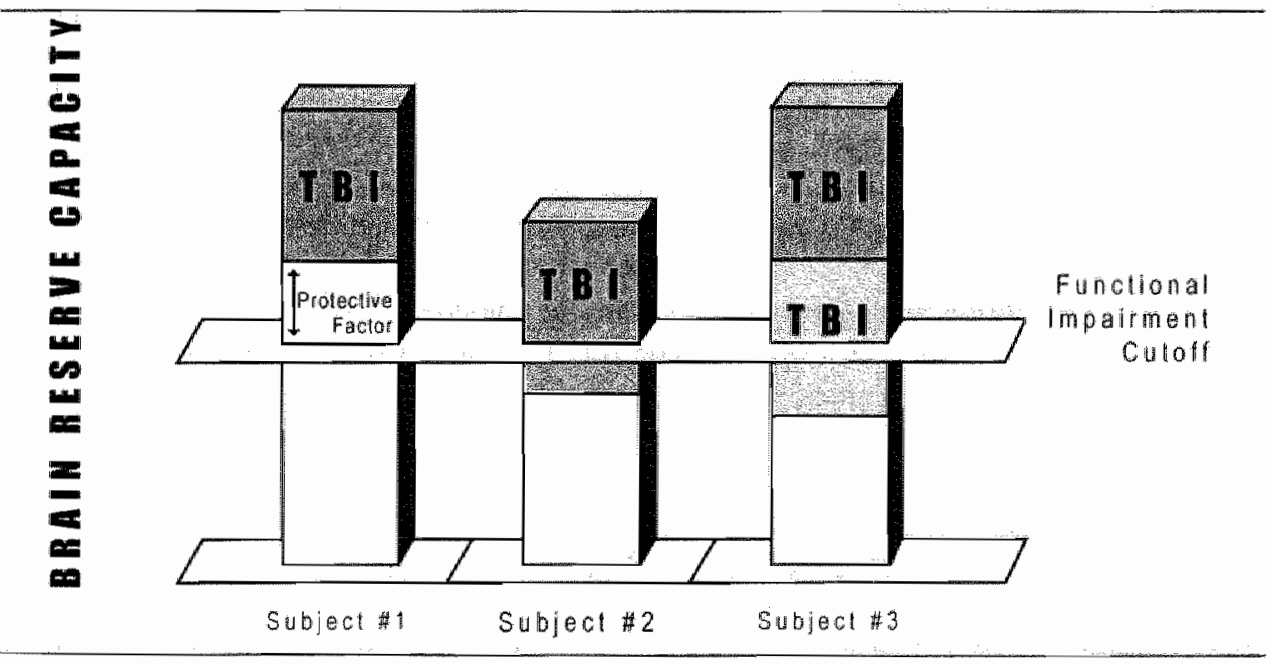

Candidate protective factors for delaying the onset of symptoms of disease states like AD are a high level of education and a strong intellect, which both are indirect measures of brain reserve 
capecity Lower educational and intellectual levels are associated with a shorter hife span and increased psychological and physical morbidity (Gurland, 1981). In the aging population, a higher level of intelligence is negatively correlated $(r=-52)$ with counts of amyloid plaques (Blessed, Tomlinson, \& Roth, 1968). Brain size may represent the possible substrate of human intelligence, since it does account for most of the variation in learning in other mammals; like mice (Jensen, 1979). Willerman, Schultz, Rutledge, and Bigler (1991) and Willerman, Schultz, Rutledge, and Bigler (1992) found a correlation of .51 between brain size and IQ when data were pooled across sexes and corrected for body size. They suggested that increased brain size might be refleeted in greater cortical size, which could reflect an inereased number of cortical columns avallable for analyzing high-noise or low-redundancy signals, eventually resulting in a more efficient information processing. A larger brain may actually prevent the development of clinically evident $\mathrm{AD}$ in the presence of histological changes of the Alzheimer type, as was demonstrated by Katzman and colleagues (1988). Lower educational level has been identified as a possible risk factor for late-onset $\mathrm{AD}$ by Chandra and colleagues (1987), who found the prevalence of low education to be much higher in patients with $A D$ than in controls. A large study in Shanghai, China, by Zhang and colleagues (1990) showed that no education was significantly related to the prevalence of dementia. The prevalence was especially high in women, possibly due to early malnutrition as well as to neuronal loss from aging, ultimately leading to a lower brain reserve capacity.

In summary, the theory based on a threshold model of cognitive aging is appealing because it is very much in accordance with the clinical observation that some older subjects appear to perform quite well although they might have sustained a significant TBI. What might seem obvious for patients with severe TBI, might also be true for milder TBI. As Mortimer and colleagues (1991) pointed out, TBI may temporarily reduce the functionality of the blood-brain barrier, resulting in loss of immunologicall protection of brain tissue and entry of toxins and/or viruses that could produce damage many years later. Secondary tissue damage may result in detectable cognitive deficits when the functional brain reserve capacity is reduced below a critical level. Mild TBI appears to accentuate the process of normal cognitive aging, and this effect is especially pronounced after multiple TBIs. The neurocognitive mechanism that might explain why "executive control functions" in particular appear to be affected by aging and TBI is discussed in the next paragriph.

\section{6 .2}

Executive Control in Mild Traumatic Brain Injury and Cognitive Aging

In this paragraph we discuss a model that explains the cognitive deficits found in mild TBI and aging in terms of changes in executive control. This model relates these changes to the neuroarchitecure underlying attentional performance. The brain locations primarily involved in attention were discussed in chapter 3 (paragraph 3.2.7). The efficiency of executive control is highly susceptible to changes in the function of the frontal lobes, and in particular to changes in the anterior cingulate gyrus (Raichle et al., 1994). As will be demonstrated, the frontal lobes appear to be compromised both in TBI and in the process of aging.

TBI is characterized by the heterogeneity of pathophysiology and consequences (Alexander, 1982; Alexander, 1987; Eisenberg \& Weiner, 1987). Therefore, it is not plausible to think in terms of a single locus of damage after TBI, although specific cognitive, behavioral, and mood disorders can directly or indirectly be attributed to frontal lobe damage (Levin \& Kraus; 1994). The horizontal and rotary movements occurring in TBI produce greater damage in areas of bony 
protrusion and rough surfaces of the skull, namely, the frontal and temporal lobes rommat Gennareli, 1974). Focal frontal lobe damage has been demonstrated by many authors, who wl mention the selective involvement of the frontal and temporall lobes (0.g., Adams et al., 1985; Clifton et al., 1980; Cullum \& Bigler, 1986; Gentry, Godersky, \& Thompson, 1988; Gentry, Godersky, Thompson, \& Dunn, 1988; Ruff et al., 1994). In diffuse axonal injury, almost any fiber tract can be damaged, although the frontal lobes are usually severely affected (Gentry et al, 1988; Nevin, 1967).

As far as aging is concerned, neuroimaging with functional and structural techniques has revealled decreases in cortical volume, increases in ventricular size and sulcal width as well as mild decreases in blood flow and glucose metabolism in nomal aging (Buchsbaum \& Siegel, 1994). Several studies have suggested that specifically the frontal lobes are susceptible to the effects of aging: microanatomical changes in the frontal cortex have been found in aged rats (Amenta et al., 1994), a decrease in MRI measures of brain volume has been detected in the frontal lobe of aging humans (Cowell et al., 1994), a decrease in glucose metabolism in frontal areas has been detected in aged humans, with the dorsal lateral frontal lobe being most affected (De Santi et al., 1995). Furthermore, slowing of prefrontal cortex function, as indicated by a significant increase of P300 latency, has been found in the elderly (Ortiz, Martin Loeches, \&. Vila, 1990). Some other studies, however, have yielded negative findings, or at least found no evidence for a specific vulnerability of the frontal lobes to aging (Peters, Leahu, Moss, \& McNally, 1994; Randolf-Swartz, Lesser, Boone, Miller, \& Mena, 1995).

\section{Figure 4.2}

Executive Control (Norman \& Shallice, 1986)

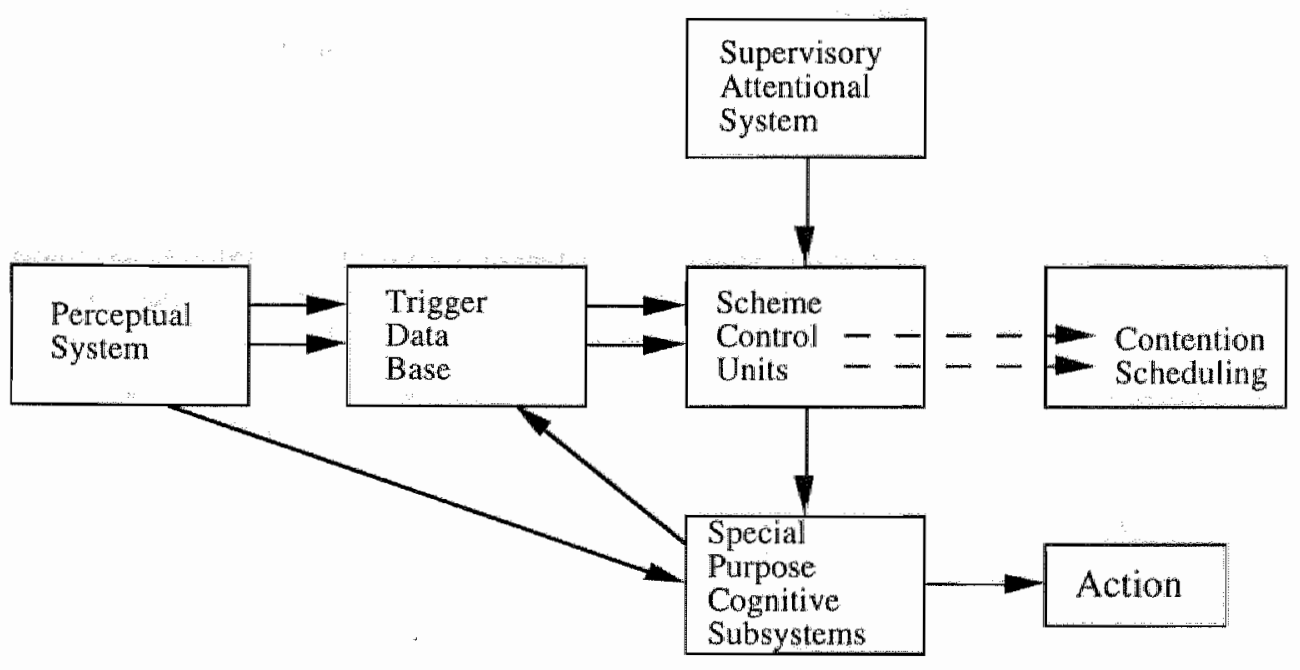

The functions of the executive, or frontal lobe, system are those cognitive abilities involved in 
the initiation, planning, and regulation of behavior (Lezak, 1993). In this way, executive functions are a type of supraordinate system that motivates self-initiated behavior, and which then gowem the efficiency and appropriateness of task performance. Executive functions are hitghly susceptible to the effects of TBI because the frontal lobes sustain most parenchymal and extraparenchymal damage induced by acceleration/deceleration injury with or without direct headstrike (Levin \& Kraus, 1994; Levin et al, 1987a). When the function of the frontal lobes is compromised by the process of aging, executive functions can expected to be affected likewise. Executive control is required when tasks involve planning, error detection, novelty, extensive processing, or conflict. The model of executive control proposed by Norman and Shallice (1986) and Shallice $(1982,1988)$ assumes several distinct subsystems of cognitive processing (see Figure 4.2). These multiple subsystems interact to coordinate goals and actions (see Allport, 1980a: Allport, 1980b) and are controlled by two qualitatively different mechanisms. Environmental information is conveyed through the perceptual system to the so-called "Trigger Data Base". Information may also be processed directly by a number of subsidiary slave systems, collectively named: "Special Purpose Cognitive Subsystems", and which are thought to be involved in specific processing (Baddeley, 1986). Schemes stored in the trigger data base are used to interpret external information in terms of relevance, novelty, and preferred subsequent actions. Specific external information triggers specific modes of action or behavior. The presence of many triggers may lead to the activation of many schemes, thus possibly causing chaotic or inappropriate behavior. The first mechanism controlling multiple external inputs operates via "Contention Scheduling", which selects one of the conflicting schemes to coordinate well-leamed behaviors and thoughts (Newell \& Simon, 1972). Compatible schemes in the trigger data base are those that have proven to be successful in attaining the goals the individual considers important. Schemes are based on previous experience and are stored in long-term memory. Once a scheme is selected, it remains active until it reaches its goal or is locally inhibited by a competitive scheme that temporarily has priority. The contention scheduling mechanism corresponds to routine selection. Desimone and Duncan (1995) have argued that visual subprocessing systems perform selection via local competition in which the receptive fields are viewed as critical resources for which stimuli are competing. They believe that the competition for these subprocessing systems is resolved via inhibition in the local neural circuit. They furthermore argued that the competition can be biased by a top down mechanism that selects objects that are impontant to the current behavior or goal. Several studies have found preferential increases in neuronal activity (Motter, 1993; Spitzer, Desimone, \& Moran, 1988) or blood flow (Corbetta, Miezin, Dobmeyer, Shulman, \& Petersen, 1991) for selected features or locations. Scalp electrical recordings have demonstrated both increases in electrical activity as a result of selected events and decreases as a result of a competing event when compared to a neutral condition (Luck, Heinze, Mangun, \& Hillyard, 1990).

In non routine situations, however, the "Supervisory Attentional System" (SAS) intervenes and provides additional inhibition or activation of the scheme appropriate for the situation. The SAS has access to the overall representation of the environment and the goals of the subject, unlike the contention scheduling mechanism, which involves only competition among subsystems. The main difference between contention scheduling and SAS is that the latter is based on strategles active in working memory, whereas contention scheduling schemes are biased by associative representations in long-tern memory. Norman and Shallice (1986) have argued that the SAS is necessary for five types of behaviors or situations in which the routine or automatic processes (Shallice, 1994) of the contention scheduling mechanisms are inadequate and the 
executive control of the SAS is called upon: a) Situations involving planning or decision-making; b) Sutuations involving error correction; c) Situations where the response is novel and not well-learned; d) Situations judged to be difficult or dangerous; and e) Situations that require overcoming habitual responses. Executive control operites only in certain situations, or during portions of tasks, when these executive functions are necessary: Executive control operations are necessary when routine functions (subsystems) are insufficient for the task at hand, or when subsystems must be overridden due to changes in goals or the environment. Executive control is not a continuous and universal process present in every cognitive activity, although these cognitive operations may play an important part in the unification of thought and action (see Alport, 1980a; Allport, 1980b). Executive control operations are suggested to be dissociated from other cognitive operations in a task (Rogers \& Monsell, 1995).

Several neuroimaging studies suggest that supervisory functions are represented by distinct frontal regions and do not exist only in the minds of Norman and Shallice (1986). These studies made use of the principle that there will be interference whenever two tasks reguire access to the same underlying systems. In the Stroop Color Word Test (Stroop, 1935), for instance, the tendency to read the name of a visual word interferes with other more arbitrary responses to that word, such as naming the ink color. Interference between tasks that use quite separate input and output pathways has been taken as a method to assess central attentional control. Activation of a midline frontal area called the anterior cingulate gyrus (see chapter 3, paragraph 3.2 .7 ) has been demonstrated with two kinds of tasks: the generate uses task (see Petersen, Fox, Posner; Mintun, \& Raichle, 1988; Petersen, Fox, Posner, Mintun, \& Raichle, 1989; Posner, Petersen, Fox, \& Raichle, 1988) and the Stroop Color Word Test (Stroop, 1935).

In the generate uses task, the subject has to give the use of a familiar word and in a control task the subject has to read or say aloud the word name. The subtraction of generate - repeat revealed three cortical areas of activation: the anterior cingulate gyus, and two left lateral areas, one area anterior to Broca's area and one in Wernicke's area (Raichle et al., 1994). When subjects repeatedly have to generate the same use, the Norman Shallice model would predict that, after practice, a scheme would be formed in the trigger data base that would be activated when the stimulus was presented and the SAS would not be necessary. After practice, Raichle and colleagues (1994) actually found that the anterior cingulate gyrus and the left lateral areas were no longer activated. Instead there was increased activation of the anterior insula which was similar to the activation found when a word was read aloud. When subjects were presented a new, unpracticed list, the anterior cingulate gyrus and left lateral areas were activated once again. Thus, the anterior cingulate gyrus is active when the SAS is is needed for appropriate behavior, according to the Norman-Shallice model, and the anterior cingulate gyrus is inactive when the Supervisory System is inactive and the contention-scheduling mechanisms are active. Neuroimaging studies of subjects performing the Stroop Colon Word Test (or Stroop-like tests) also showed anterior cingulate gyrus activation when subjects had to give a response that was not practiced and thus could not trigger a scheme stored in long-term memory (see Bench et al., 1993; George et al., 1994;. Pardo, Pardo, Janer, \& Raichle, 1990; Taylor, Kornblum, Minoshima, Oliver, \& Koeppe, 1994). These studies, however, differed in the precise locations of the anterior cingulate gyrus that were activated. George et al. (1994) suggested that different portions of the cingulate are involwed in different aspects of the processing of incongruent tasks. Their results suggested that the anterior cingulate gyrus is active during tasks that require some thought, and is inactive or less active as tasks become routine, as in the case of reading 
words alovd or following practice generating the same use.

Lesions of the fronal lobe often produce disorganized or incoherent behavior (Duncan, Burgess, \& Emslie, 1995; Shallice \& Burgess, 1991a; Shallice \& Burgess, 1991b). In neuropsychology, the dysexecutive syndrome following TBI or AD involves the loss of the ability to plan coherently, to solve problems, or to organize daily life. Patients suffering from this syndrome have difficulty with problem solving tasks such as the Tower of London (Shallice, 1982), in which planning ahead is an important component: Shallice (1982) found that patients with left frontal lesions performed poorly on this task. Several studies of TBI patients, however, have provided little evidence for the presence of deficits of supervisory attentional control, but ample evidence for the presence of a deficit in speed of information processing (Ponsford \& Kinsella, 1992; Van Wolfelaar, Brouwer, \& Van Zomeren, 1990). This, however, might suggest that only damage to specific, critical areas of the frontal lobes results in poor performance on tasks requiring frontal lobe function or that the extensive connections of the frontal lobes with other cortical and subcortical areas may be important to successful planning. As early as 1886, Ferrier warned against a modular approach to cognitive impairment after brain damage. Cognitive neuropsychologists generally assume that damage to one component of the functional architecture will have exclusively local effects (Farah, 1994). Several neurocognitive tasks have been developed in line with this assumption. The Wisconsin Card Sorting Test (WCST; Berg. 1948; Grant \& Berg, 1948) and the Halstead Category Test (Halstead, 1947; Reitan Wolfson, 1985) are tests that purportedly assess functions associated more with the frontal lobes than other lobes. Recently, however, several studies have questioned the specificity of these tests. (Arnett et al., 1994; Barkley \& Grodzinsky, 1994; Grattan, Bloomer, Archambault, \& Eslinger, 1994; Van den Broek, Bradshaw; \& Szabadi, 1993). The most compelling evidence comes from studies by Anderson, Bigler, and Blatter (1995) and Anderson, Damasio, Jones, and Tranel (1991). They found no significant differences in WCST performance between TBI patients with frontal versus non-frontal damage, as evidenced by MRI. In the study of Anderson and coworkers (1991) some patients with extensive frontal lobe damage performed well on the WCST and some patients with damage outside the frontal Jobes performed inadequately. Anderson and coworkers (1995) therefore concluded that frontal Jobe tests do not say anything about frontal lobe integrity and netropsychological functioning in TBI patients. Frontal lobe dysfunction in TBI and aging should therefore be at least partially dissociated from frontal abnormalities. After TBI, attention should also be directed to the effects of pain, fatigue and general illness, since all of these may lead to a lowered ability to control, select, analyze, and shift attention.

In conclusion ${ }_{4}$ it is important to distinguish between the components of executive control in order to provide a more analytic treatment at the cognitive and anatomical levels The analysis of executive function as a unified aspect of attention has progressed substantially in terms of both cognitive criteria and brain function. In the future some of these functions may be found to be associated with particular structures. Executive control raises the problem of the homunculus, but as Atmeave (1959) first suggested, progress is made by dissociating operations from the overall homunculus one component at a time. It may eventually be possible to understand to what extent executive attention can be considered a unitary system or a more distributed system. 
In most patients with mild TBI, postconcussional symptoms disappear in tu matter of weeks or months, though the length of time before normal netropsychological scores are reached will depend on the measures that are used. Although patients with mild TBI may appear to have made a full recovery (i.e., returned to preinjury social and work life), they may still show persistent impairment when exposed to metabolic (e.g., hypoxia) or cognitive stress (e.g., dual-tasks) or when they reach advanced age. Longer than normal recovery times are observed in older patients and in patients who have had a previous TBI. Because of the progressive loss of brain tissue with adwancing age, older people already have a reduced information processing. capacity. This reduction in capacity is consequently further aggravated by brain injury. After injury, older patients usually recover more slowly and sometimes incompletely. Patients with multiple TBIs show a similar pattern, which also has been attributed to the effects of neuronal loss. These multiple injuries are recognized as a major risk factors for $\mathrm{AD}$ later in life.

A useful model for predicting outcome after mild TBI is a model which assumes the presence of brain reserve capacity. Due to loss of neuronal function with age, the brain reserve capacity is reduced, as is also the case in (mild) TBI. The cumulative effects of aging and TBI appear to cause previously subthreshold symptoms to become evident.

There is ample evidence that several aspects of attention decline with aging and with mild TBI. Up till now, however, it was not clear whether the combination of physiological aging and mild TBI leads to accelerated cognitive aging, although studies on severe TBI suggest that this is the case. The resemblance in the kind, but not in the extent, of neuropathology in patients with mild, moderate, and severe TBI supports this notion. It is also possible that physiological aging and mild TBI merely add up, without causing an increase in the speed at which subjects age, but rather making him or her a few years older. A person who sustained a mild TBI at age 40 , for instance, may show a cognitive performance that is more appropriate for a subject of 60 years. Finally, it is possible that the effects of mild TBI disappear with the passage of time. The common belief is that brain damage is irreversible, and that because neural tissue is incapable of regeneration, damage to neural structures is permanent and permanently disabling. In fact, that is only partly true. Whether a subject recovers completely from mild TBI is likely to depend on the age at which the mild TBI is sustained and the resources the subject can allocate to compensate for the cognitive functions that are compromised. Protective factors that enhance brain reserve capacity are a higher intellectual or educational level, and these are potentially important factors in predicting the outcome of the rehabilitation process after head injury Patients who do not have these resources readily available should be offered a more supportive environment that in part compensates for the diminished cognitive functions of these patients.

\section{8}

\section{References}

Absher, J. R., \& Cummings, J. L. (1994). Cognitive and noncognitive aspects of damentia syndromes: An overview. In A. Bums \& R. Levy (Eds.), Dementia, (pp. 59-76). London; Chapman \& Hall .

Adamovich, B. B., Henderson, J. A., \& Auerbach, S. (1985). Cognitive Rehabilitation of Head lwinged Patients. San Diego, CA: College Hill Press.

Adams, J. H., Doyle, D. Graham, D. I., Lawrence, A. E., McLellan, D. R., Gennareli, T. A., Pastuszko, M., \& Sakamoto, T. (1985). The contusion index: A reappraisal in thuman and experinental non-misgile head injury. Neuropathology and Applied Neurobiology, $/ /(4), 299-308$. 


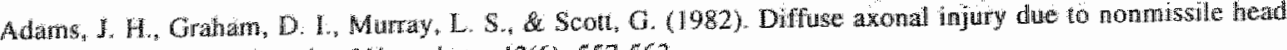
injury in humans. Anmals of Nearology, $12(6), 557.563$.

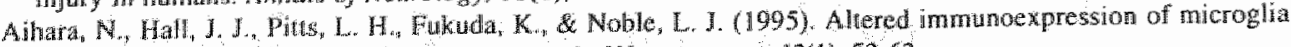

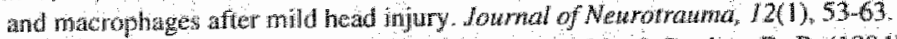

Alexander, M. J., Martin, N. A. Khannd, K., Caron, M. Becker, D. P. (1994) Regional cerebral blood flow

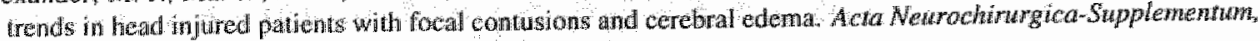
$60,499-481$.

Alexander, M. P. (1982), Tramatic bran inury, In D. F Benson \& D. Blumer (Eds), Psychiorric Aspects of Nenpologic Disease, (Vol. 2 pp. 219.250 ) Wew York: Grune \& Straton.

Alexander, M. P. (1987). The role of neurobehaviond syndromes in the rehabiliation and outcome of chosed head

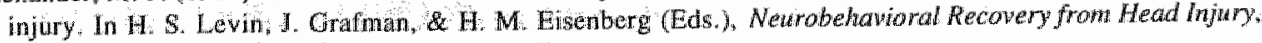
(pp. 191-205). New York: Oxford Unversity Press.

Alexandre, A, Colombo, F. Nertempi, P. \& Benedetil, A. (1983). Cognive outcone and early indices of severity of head injury. Journa of Nemosurgery, 59,5), 751-761

Aliport, D. A (1980a). Alention and performance. New directions. In G. Claxton (Ed), Cognitive Psychology. (pp. 12 153). London Routledge \& Kegan Paul.

Allport, D. A. (1980b). Patterns and actions: Cognitiwe mechamisms are content-specific. In C. Claxton (Ed.). Cognhive Pychology. (pp. 26-64). London: Routledge \& Kegan Paul

Alves, W. M. Colohan, A. R. O'Leary, T. 1., \& Rimel, R. W. (1986). Understanding postratimatic symptcoms aller nino head injury. Jownal of Head Trawna Rehabilitation, I(2), 1-12.

Amaducci, L. A., Frotiglioni, L. Rocoa, W. A. Fieschi, C., Limrea, P.., Pedome, D., Bracco, L." Lippi, A., Gandollo, $C_{n}$, Bino, G., Prencipe, M., Bonati, M. L., Girotli, F, Carella, F., Tavalato, B.; Fera, S, Lenzi, G. L, Carolei, A., Gambi, A. Grigoletto, $F^{\circ}$ \& Schoenberg. B. S. (1986). Risk factors for clinically

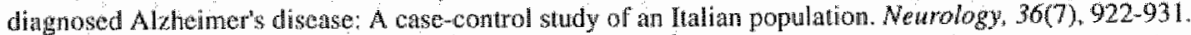

Amenta, F, Bograni, S., Cadel, S., Ferrante, F., Valsecchi, B., Vega, J. A. (1994) Microanatomical ahanges in the frontul cortex of aged rats: Effect of L-doprenyl treatment. Brain Research Bullerin, 34(2), 125-131.

American Psychiatric Association (1994). Diagnostic and Statisticat Mannal of Mental Disorders. (Fourth ed.). Washinghon, DC: American Psychiatric Association.

Anderson, C. V., Bjgller, E, D., de Blater, D. D. (1995). Frontal lobe lesions, diffuse darnage, and neuropsychological functioning in traumatic brain-injured patients. Joumal of Chical and Experimental Newropsychology, $/ 7(6), 900-908$.

Anderson, S. W. Damasio, H., Jones, R. D. \& Tranel, D. (1991). Wisconsin Card Sorting Test performance as a medsure of frontal lobe damage. Jounal of Cinical and Experimenfal Nearopsychology, 13(6), 909-922.

Arcia, E. \&ualtieri, C. T. (1993). Association between patient report of symptoms after mill head injury and nesrobehavioural performarice. Brain Whury, $7(6), 481-489$.

Arnet, P. A., Rac, S. M., Bernardin, L. Grafman, J., Yetkin, F, Z, \& Lobeck, L. (1994) Relationship between fromal lobe lesions and Wisconsin Card Sorting Test performance in multiple sclerosis. Nearology, $44(3), 420-425$

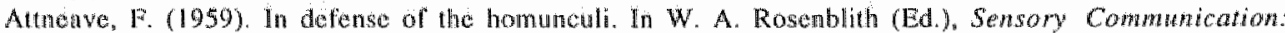
Contributions to the Symposium an Principles of Sensory Conmunication, (pp. 777.782). Cambridge, MA: MTT Press.

Azmilia, E. (1987). Who CNS serotomergic system: Progression toward a collaborative organization. In H. Y. Meltae (Ed.), Psychopharmacology: The third Generation of Progress. (pp. 61-74). New York: Ravon Press.

Baddeley, A. (1986). Working Menory. London: Oxford University Press.

Baddeloy" A. Logle, R., Bressi, S., Sala, S. D. \& Spinnler, H (1986). Dementia and working memory. Quarew How wal of Lxperimental Psychology: Human Experimental Psychology, 38(4), 603-618.

Barkloy, R. A. B Grodzinsky, G. M. (1994). Are tests of frontal Iobe functions useful in diagnosis of attention deficit disonders? The Chincal Neuropsychologist, $8(2), 121-139$.

Barth, J. "T., Alves, W. M., Ryan, T. V., Macciocchi, S. N, Rumel, R. W., Jane, J. A., d Nelson, W. R. (1989). Mild head injury in sports: Neuropsyohological sequelae and recovery of fumction. In H. S. Levin, H. M. Fisenberg, \& A. L. Benton (Eds.), Mild Head Mjury, (pp. 257-275). New York: Ox ford University Press.

Burth, I. T. Macciochi, S. N., Giordeni, B., Rimel, R., Jane, J. A. \& Boll, T. I. (1983). Neuropsychological sequel ate of minot head injury. Neurasurgery, /3(5), 529-533.

Batchelor, J. Harvey, A. G., \& Bryant. R. A. (1995). Stroop Colotr Word Test as a measure of attentional delioit following mild head injury. Chical Nerpopsychologist, 92 ), 180-186.

Beers, S. R. (1992). Cogntiwe offects of will head injury in children and adolescents. Neuropsychology Reviews $3(4), 281-320$ 


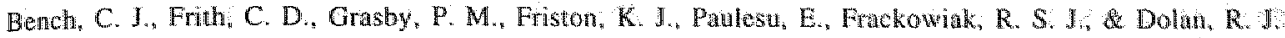

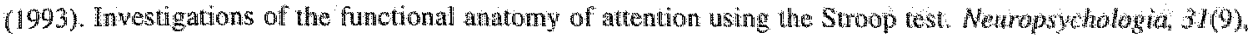
907-922.

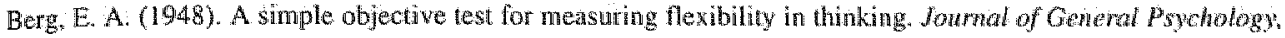
$39,15-22$.

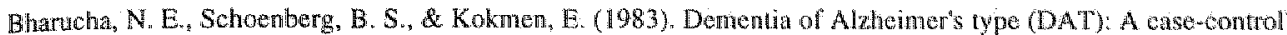
study of association with medical conditions and surgical procedures. Neurofogy, $33(5 u p p 1,2), 85$.

Bigler, $\mathbb{E}$. D., \& Snyder, 1. L. (1995). Neuropsychological outcome and quantiative netroumaging in mild head injury. Archines of Clinical Newropsychology, 10(2), $159 \% 174$.

Binder, L. M. (1986). Persisting symptoms after mild head injury: A review of the postooncussive syndrome. Joumal of Clinical and Experinemal Nearopsychology, $8(4), 323-346$.

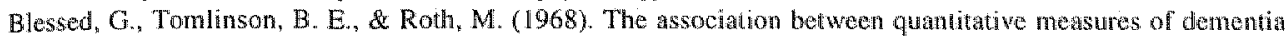
and of senile change in the cerebral grey natter of elderly subjects. British Joumal of Prychiath $1 / 4(512)$, $797-811$.

Blumbergs, P. C., Scott, G., Manavis, J, Wainwright, H., Simpson, D. A. \& McLean, A. J. (1994). Staining of anyloid precursor protein to study axonal dannge in mild head injury. Lawcet, 3448929), 1055-1056.

Blumbergs, P. C. C... Jones, N. R., \& North, I. B. (1989). Diffuse axonal injury in head tratuma. Jowmal of Neurology, Neurosurgery, and Psychiatry, $\$ 2(7), 838-841$.

Bohner, N., \& Jolles, J. (1992). Neurobehavioral aspects of postconcussive symptoms after mild head injury. Journal of Nernous and Mental Disease, $180(11,683-692$

Bohnen, $\mathbb{N}_{n}$, Jolles, J., \& Twijnstra, A. (1992a). Modification of the Stroop Color Word Test inprowes differentiation between patients with mild head injury and matched controls. Chnical Neuropweholowist, $6(2)$, $178-184$.

Bolmen, N., Twijnstra, A., \& Jolles, J. (1992), Performance in the Stroop color wond test in relationship to the persistence of sympioms following mild head injury. Acta Nentologica Scandinawica, 85(2), 116,121 .

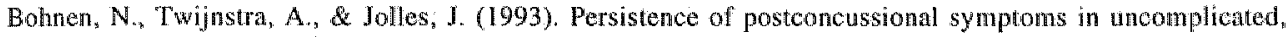
mildly head-injured patients: A prospective cohort study. Newropsychiaty Newropsycholog, and Behavioral Neurology, $6(3), 193-200$.

Bohnen, N., Twijnstra, A., Kroeze, J., \& Jolles, J. (1991). A psychophysical method for assessing visual and acoustic hyperaesthesia in patients with mild head injury. British Joumal of Psychiatry, 159(12), 860-863.

Bothen, N, Twijnstra, A., Wijnen, G., \& Jolles, J. (1991) Tollerance for light and sound of paticnts with persistent post-concussional symptons 6 months after mild head injury. Jounal of Newrology, 238(8), 443. 446 .

Bolnnen, N., Twijnstra, A., Wijmen, G., J Jolles, J. (1992). Recovery from visual and acoustic hypernesthesin after mild head injury in relation to pattems of behavioural dysfunction. Jow rmat of Neurology, Newosturgery, and Psychictry, $55(3), 222-224$

Bohnen, W. Vari Zutphen, W., Twijnstra, A., Wijnen, O., Bongers, Ju, Jolles, J. (1994). Lante outeome of mild head injury: Results from a controlled postal survey. Brain Ming: $8(8), 701.708$.

Bolner, N. 1., Jolles, J., \& Twijnstra, A. (1992b), Neuropsychological deficis in patients with persistent symptoms six months after mild head injury. Neurosurgery, 30(5), 692-696.

Bohnen, N. I., Jolles, J., Twijnstra, A., Mellink, R, \& Winen, G. (1995). Late nourobelawiounal symploms afier mild head injury. Brain Injary, $9(1), 27.33$.

Bourke, R. S. (1988). Head Injwy. Bethesda, MD: NIWCDS.

Brak, H., \& Brak, E. (1991). Neuropathological stageing of Altheimer relatect changes. Acha Newopathologica, $82(4), 239-259$

Broe, G. A., Henderson, A. S, Creasey, H., McCusker, E, Ronten, A. E., Jom, A. F., Longley, W., \& Anthony, J. C. (1990). A case-control study of Aldeimer's disease in Australia. Newrology, 40(1). 1.698. 1707 .

Brouwer, W. H., \& Van Wolffelar, P. C. (1985). Sustaimed attention and sustained effort after closed head injury: Detection and $0.10 \mathrm{~Hz}$ heart rate variability in a low event rate vigillance lask. Cortex, $2 /(1), 11 \mathrm{I} / 19$.

Brown, S. J., Fann, J. R. \& Grant, L. (1994). Postconcussionall Disorder: Thine to acknoumledge a common

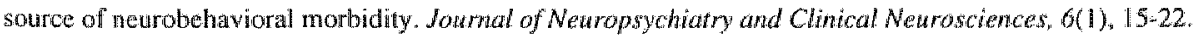

Buchsbaum, M. S. \& Siegel, B. V. (1994). Neuromaging and the aghe process in psychiatry. Anremationul Review of Psychiatry, 6(1), 109-118.

Buchtel, H. A. (1987). Attention and vigilance after head injury, In H. S. Levin, J. Crafman, \&. M. Eisenberg (Eds.), Neurobehavional Recovery from Head Injmy, (pp. 372 378), New York: Oxford Universily Press. 


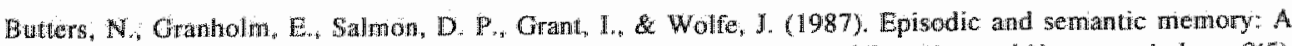
comparison of amneste and demented pathents. Joumal of Chncal and Experimental Neuropsychology, $9(5$. 479.497.

Carlson, A. (1987). Monoamines of the CNS: An historical perspective. In H. Y. Meltzer (Ed.), Psychopharmacology: The third Geveration of Progress, (pp. 39-48). New York. Raven Press.

Cartsen, C. S., Svardsudd, K. \& Welin, L. (1987). Long tem effects of head injuries sustained during life in three male populations. Journal of Neurosirgery, 672 (2), 197-205.

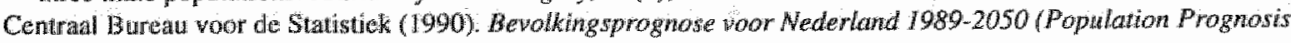
for the Netherlands 1989-2050). Voorburg. The Netherlands: CBS.

Central Buteau voor de Statistiek (1993). Statistisch Jaarboek 1993 (Statistical Yearbook 1993). Voorburg. The Netherlands: CBS.

Chandra, V., Kokmon, E; schoenberg, B. S. (1987). Head injury with loss of consciousness as a risk factor for Alzheimer's disease using prospectively collected data. Nearology, 37(Suppl. 1), 152.

Chandra, V., Kokmen, E. Schoenberg, B. S., \& Beard, C. M. (1989). Head trauma with loss of consciousmess as a risk factor for Alzheimer's disease. Newrology. 39(12), 1576. 578 .

Chandra, V., Philuposs, $V_{4}$, Bell, P. A., Lazaroff, A., Schoenberg, B. S. (1987). Caste-control study of late onset 'probable Alzeimer's disease'. Neurology, 37(8), 1295-1300.

Cicerone, K. D., Kalmat, K. (1995). Persistent postconcussion syndrome: The structure of subjective complarits after mild haumatic brain injury, Jourhal of Head Trama Rehabilitation, 10(3), 1- 17.

Clifton, G. L., Grossman, R. G., Makela, M. E. Miner, M. E., Handel, S., \& Sadhu, V. (1980). Neurological course and comelated computerized tomography findings after severe closed head ingury. Journal of Newrosungery; $52(5), 611 \ldots 24$

Cllfton, G. L., Ziegler, M. G. a Grossman, R. G. (1981), Circulating catecholamines and sympathecic activity after head injury. Neurosurgery. 8(1), 10\%14.

Clinton, J., Ambler, M. W., \& Roberts, G. W. (1991). Post-traumatic Altheimer's disease: Preponderance of a single plaque type. Neuropatholology and Applied Neurabiology. 17(1), 69.74.

Corbetta, M., Miezin, F, M., Dobmeyer, S., Shulman, G. L., \& Petersen, S. E. (1991). Selectiwe and divided attention during visual discrimination of shape, color, and speed: Funcional anatomy by positron emission tomagraphy. "Jownal of Neuroscience, $/ 1(8), 2383-2402$.

Corsellis, J. A. N., \& Brierly, J. B. (1959). Obserwations on the pathology of insidious dementia following head injury. Tournal of Mental Science, $105,714-720$.

Corsellis, J. A. N., Bruton, C. J., \& Freeman-Browne, D. (1973). The aftermath of boxing. Psychological Medicine, 3(3), 270-303.

Cowell, P. E., Turetsky, B. I., Gur, R. C., Grossman, R. 1., Shtase1, D. L., \& Gur, R. E. (1994). Sex differences in aging of the human frontal and temporall lobes. Joumal of Neuroscience, 14(8), 4748-4755.

Cremona-Meteyard, S. L., Clark, C. R, Wright, M. J., E Geffen, G. M. (1992). Covert orientation of visual attention after closed head injuiry. Neuropsychologia, $30(2), 123 \omega 132$.

Ctemona-Mateyard, S. Lo, \& Geffen. O. M. (1994). Persistent visuospatial attention deficits following mild head injury in Australian Rules football players. Nentopsychologia, 32(6), 649-662.

Crof, A. C. (1995). Biomechamics. In S. M. Foneman \& A. C. Croft (Eds.), Whiplast Injuries. The cervical Acceleratiow/Decelteration Syadrome, (2nd ed. pp. 1-92), Baltimore, MD: Williams \& Wilkins.

Cullum, C. M., \& Bigler, E. D. (1986). Ventricle size, cortical atrophy and the relationship with netinopychologicil stalus in closed head injlery: A quantitative analysis. Journal of Clinical and Experimental Newropsyckology, 8(4), 437-452

Cunmings, J. L., \& Benson, D. F. (1992). Dementia: A climical Approach. (2nd ed.). New York: Butterworths.

Curry, $S_{3}$ H. (1980). Event-related potentials as indiconts of structural and functional damage in closed head injury. Progress in Brain Research, $54,507-515$.

Dacey, R, G. Dikmen, S. S., Temkin, N., MeLean, A., Amsden, G., W Winn, H. R. (1991). Relative elfects of brain and non-braim injuries on neuropsychological and psychosocial outcome. Journal of Trama. 3 I(2), 217-222.

Davies, D. R. de Patasuraman, R. (1982). The Psychology of Vigilance. London: Academie Press.

De Santi, S. De Leon, M. I, Convit, A., Tarshish, C., Rusinek, H., "Tsui, W. H., Sinaiko, E. Warg, G. J., Bartat. E... \& Volkow. N. (1995). Agearelated changes in brain. II. Positron emission tomography of frontal and temporal lobe glucose metabolism in normal subjects. Psychictric Quarteriy, 66(4), 357-370

DeKosky, S. T. \& Scherf, S. W. (1990). Synapse loss in frontal cortex biopsies in Alrheimer's disease: Correlation with cognitive severity. Amnals of Nerurology, 27(5), 457-464. 
Deland, N., Vanier, M., Lambert, J, \& Provost, J. (1992). A study of focused attention ib sevelely head-injured

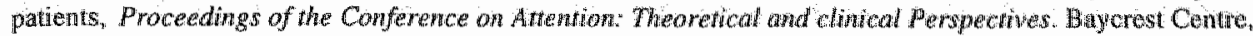
Toronto, Canada.

Dencker, S. V. (1958). A follow-up study of 128 closed head injuries in twins wsing co-fwins as controls, Acto Pstoliantica Neurologica Scandincuica, 33(Suppl. 123), 123-125.

Denker, P. G. (1944). The postconcusion syndrome: Prognosis and evaluation of the organic factors. New York State Medicine Joural, 44, 379-384.

Department of Transport (1995). Transpont Statistics Great Brinain. London: HMSO.

Desimone, $\mathrm{R}$. \& Duncan, J. (1995). Neural mechanisms of selective artention. Aimual Review of Neuroschence, $18,193-222$.

Dikmen, S., McLean, A., Remkin, N (1986). Neuropsychological and psychosocini consequences of minon head injury. Jowmal of Neurology, Neurosurgery, and Psychiary, 49(11), 1227-1232.

Dikmen, S., Melean. A., Temkin, $\mathrm{N}_{\text {, }}$ \& Wyler, A. (1986). Neuropsychologic oulcome at one-rnonth postinjury. Ardives of Physical and Medical Rehabilitation, 67(8), 507-513.

Dikmen, S., Temkin, N. McLean, A., Wyler, A." \& Macharner, J. (1987). Memory and head injury severity. Joumal of Neurology. Newroswrgery, and Psychiatry, 50(12), 1613-1618.

Dikmen. S. S., \& Levin, H. S. (1993). Methodological issues in the study of mild head injury. Jownal of Heat Trauma Rehabilitation, 8(3), 30-37.

Dikmen, S. S., Machaner, J., Temkin, N., \& McLean, A. (1990) Neuropsychological recovery in patients with moderate to severe head injury: 2 year follow-up. Journal of Chical and Experinental Neuropsychology. $12(4), 507-519$

Dikmen, S. S., Machamer, J. E., Winn, H. R., \& Temkin, N. R. (1995). Neuropsychological outcome at l-year post head injury. Neuropsychology, 9(1), 80-90.

Dixon, C. E., Taft, W. C. \& Hayes, R. L. (1993). Mechanisms of mild traumatic brain injury. Jounal of Hed Trauma Rehabilitation, $8(3), 1-12$.

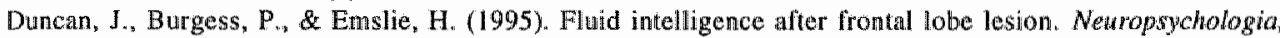
$33(3), 261-268$.

Ebbs, S. R., Beckley, D. E., Hammonds, J. C., Teasdale, C. (1986). Incidence and duration of neck pain among patients injured in car accidents. British Medical Monmal, 292, 9495.

Edna, T. H., \& Cappelen, J. (1987). Late post-concussional symptomis in traumatic head injury. An analysis of frequency and risk factors. Acta Neurochinurgica Wien, $86(1-2), 12-7$.

Eisenberg, H. M., \& Levin, H. S. (1989). Computed tomography and magnetic resonance imaging in mild to moderate head injury. In H. S. Levin, H. M. Eisenberg, \& A. L. Benton (Eds.). Mild Head Injury, (pp. 133. 141.). New York: Oxford University Press.

Eisenberg, H. M., \&einer, R. L. (1987). Input variables: How information from the acute injury can be used to characterize groups of patients for studies of outcome. In H. S. Lovin, I. Crafman, \& H. M. Einonberg (Eds.). Neurobethawiaral Recowery from Head hnjury, (pp. 13-29). New York: Oxford University Press.

Elson, L. M., \& Ward, C. C. (1994). Mechanisms and pathophysiology of mild hend injury. Sumincits in Newrology; $14(1), 8-18$.

Esselman, P. C. Uomoto, J. M. (1995). Classification of the spectrum of mild traumatic brain injury. Bran Irjury. 9(4), 417-424.

Evans, $\mathbb{R}$. W. (1992a). Mild trammatic brain injury. Physical Medical and Rehabilitation Clinics of Nonth America, 3, 427-439.

Evans, R. W. (1992b). The postconcussion syndrome and the sequelae of mild head injury. Neurologic Clinics, $10(4) .815-847$.

Ewing, R., MoCarthy, D., Gronwall, D., \& Wrightson, P, (1980). Persisting effects of minor head injury observable during hypoxic stress. Jowmal of Clinical Newropsychology, 2, 147-155.

Farah, M. J. (1994). Neuropsychological inference with an interactive brain: A critique of the "locality" assumption. Behavional and Brain Sciences, $17(1), 43 \% 104$.

Farrar, L. A., O'Sullivan, D. M., Cupples, A., Crowdon, J. H. \& Myers, R. H. (1989). Assessment of genetic risk for Alzheimer's disease among first-degree relatives. Antals of Neurology, 25, 485-493.

Fenton, G., McClelland, R., Montgomery, A., MacFlynn, G., \& Rutherford, W. (1993). The postconcussional syndrome: Social antecedents and psychological sequelae. British fowmal of Psychiatry, 162, 493-497.

Ferini-Strambi, L., Smirne, S., Garancini, P. Pinto, P., \& Franceschi, M. (1990). Clinical and epidemiological aspects of Alzheimer's disease with presenile onset: A case control study. Newroepiderwology, 9(1), 39-49.

Ferrier, D. (1886). The Functions of the Brain. Smith: Elder. 
Filoted, J. V., Delis, D. C. Masmina, P. J., Demexdura, T., Buners, N., \& Samon, D. P. (1992). Directed and

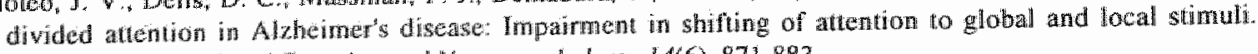

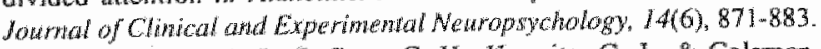

Flood. D. G, Buell, S. J, Defiorc, C. H., Horwitz, G. J, \& Coleman, P. D. (1985). Age-related dendritic growth in dentate gyrus of human bran is followed by regression in the "oldest old". Brain Research, 345(2), $366-368$.

Frankowski, R. F., Annegers, J, F, \& Whiman, S. (1985). The deseriptive epidtemiology of head tratuma in the United Statef. In D. P. Becker J. T. Povlishock (Eds.), CNS Trauma Statws Report (pp. 33-43). Bethesda, $\mathrm{MD}: \mathrm{NINCDS}, \mathrm{NIH}$.

French, L. R. Schuman, L. M., Mortimer, J. A., Hutton, I. T., Boatman, R. A., \& Christians, B. (1985). A catse-control study of dementia of the Alzhe mer lype. American Jormal of Epidemiology, $12 /(3), 414 \times 421$.

Galasko, C. 5., Murray, P. M. Pitcher, M., Chambers, H. Mansfield, S. Madden. M. Jordon, C., Kinsella, A., \& Hodgon, M. (1993). Meck sprains after road traffic accidents: A modem opidemic. Mary, 24(3), 155157.

Geddes, I. F., Vowles, G. H., Robinson, S.F. D., Sutcliffe, J. C. (1996). Neurofibrillary tangles, but not Alzheimer-1ype pathology, in a young boxer. Neuropathology and Applied Newrobiology, 22(1), 12- 6.

Gennarell\%,

Gentarelli, T. A. Adams, J. H., \& Graham, D. 1. (1986). Diffuse axonal injury: - A new conceptual approach to an old problem. In A. Baethman, K. G. Go, \& A. Unterberg (Eds.) Mechanisms of secondary Brain Damage, (pp. 15-28). New York: Plenum.

Gennarelli, T. A., Spieman, G. M. Langfitt, T. W., Gildenberg, P. L., Harrington, T., Jane, J. A., Marshall, L. P. Miller II. D, \& Pits, L.H. (1982). Influence of the type of intractanial lesion on outcome from severe head injury. Journat of Neurosurgery, $56(1), 26-32$

Gentilini, M., Nichelli, $P_{n}$ \& Schoenhuber, R. (1989). Assessment of antention in mild head injury. In H. S. Lewin, H. M. Eisenberg, A. L. Benton (Eds.), Mild Head Mjury, (pp. 163-175). New York: Oxford Uniwersity Press.

Gentilini, M., Nichelli, P., Schornhuber, R., Bortoloti, P., Tonelli, L., Falasea, A., \& Merli, G. A. (1985). Neuropsychological evaluation of milat head injury. Hounal of Neurology. Nenrosurgery and Psychiatry, $48(2), 137-140$.

Gentry, L. R., Godersky, J. C., \& Thompson, B. (1988). MR maging of head tramma: Review of the distribution and adiopathologic features of atumatic lesions. American Joumal of Roentgenology $150(3)$, 663-672.

Geniry, L. R., Godersky, J. C., Thompson, B., \& Dunn, V. D. (1988). Prospective comparative study of intermediate-field $\mathrm{MR}$ and $\mathrm{CT}$ in the evaluation of olosed head trauma. American Joumal of Roengenology. $150(3), 673-682$

George, M. S., Ketter, T. A., Parekh, P. I „, Rosinsky, N., Ring, H., Casey, B.J, Trimble, M. R, Horwitz, B... Herscovitch, P., Post, R. M. (1994). Regronal braim activity wher selecting response despite interference: An H2150 PQT study ol the Stroop and an enotional Stroop. Howm Brown Mopping, J(3), 194.209.

Gimota, S. L., Weiner, J. M., \& Karnaze, D. (1987). Prognosis and outcome in severe head injury, In P. R. Cooper (Ed.). Head injory, (pp. 464-487). Baltmote: Wiliams and Wilkins.

Goldstein, M. (1990). Trumatio brain injury: A silent epidente (editorial). Annals of Newology, $27(3), 327$.

Crant, D. A. w Berg. E. A. (\$948). A behavional analysils of degree of reinforcement and ease of shifting to newe responses in a Woigl-Hype card-sorting problem. Jom nal of Experinental Prychology, 38, 404-411.

Gratan, L. M., Bloomer, R. H., Archambaul, F. X., \& Eslinger, P. J. (1994). Cognitive fexibility and enpathy after frontal lobo lesion. Neuropsychitry, Neuropsychology, and Behavioral Newrology, 7(4), 251259.

Graves, A. B., White, E., Koepsell, T, D., Reitler, B. V., van Belle, G., Larson, E. B., \& Raskind, M. (1990). The associanot between head trama and Alxheimer's disease. American Journal of Epidemiology. 13/(3), 491-501.

Gronwall, D. (1976). Pertormance changes during recovery from closed head injury. Proceedings of the Anstratian Association of Newrology, 13, 143-147.

Gironwall, D, (1987). Adwances in the assessment of attention and information processing after head injury. In $\mathbb{H}$. S. Levin, I. Grafman, \& H. M. Eisenberg (Eds.), Newrohehawiaral Recovery from Head lnjury. (pp. 355-371). New York: Oxford University Press.

Gronwal, D. (1989). Cumulative and persisting effects of concussion on attention and cognition. In $H$. S. Levin, H. M. Eisenberg. \& A. L. Benton (Eds), Mild Head Injury, (pp. 153-162). New York: Oxford University Pross. 
Gromwall, D. (1991). Minor head infury. Neuropsychology $5(4), 253265$

Gronwall, D. Wrightson. P. (1974). Delayed recovery of intellectual function after minor head injurg, Lawner, $2(7881), 605-609$.

Gronwall, D. \& Wrightson, P. (1975). Cumulative effect of concussion. Lantet, 2(7943), 995-997.

Gronwall, D., \& Wrightson, P. (1980). Duration of post tratuatic amnesia after mild head injury. Joumal of Clinical Neuropsychology, 2, 51-60.

Gronwall, D, \& Wrightson, P. (1981). Memory and information processing after minor head injury. Wownal of Neurology. Newoswgery and Psychatry, 44(10), 389-895.

Gronwall, D. M. (1977). Paced auditory serial-addition lask: A measure of recovery from concussion. perceptual and Motor Skils, 44(2), 367-373.

Gronwall, D. M. A., \& Sampson, H. (1974). The Psychological Effects of Concusaion. (Vol. 2). Auekland. New Zoaland Auck and University Press.

Gualtieri, C. T. (1990). Neuropsychiary and behaviowal pharmacology. Berlin: Springer Verlag.

Gualien, C. T. (1995). The problem of mild brain injury. Newropsychary, Newopsychology, and Bethanonat Newrology, $8(2), 127-136$.

Gurland ${ }_{\mathrm{s}}$ B. J. (1981). The borderlands of dementia: The influence of sociocultural characteristics on the rat tos of dementia occuring in the senium. Im N. E. Miller Q G. D. Cohen (Eds.). Aspects of Alweimer's Disedse and Senile Denentia, (pp. 61-80). New York: Raven Press.

Hagnell, O., Lanke, J., Rorsman, B., Ojesjo, L. (1981). Does the incidence of age psychosis decrease? A prospective, longutudinal study of a complete population investignted during the 25-year period 1947-1972: The Lundby study. Neuropsychobiology, 7(4), 201-211.

Halstead, W. C. (1947). Brain and Intelligence: A Quantictive Sudy of the Fromtal Loben. Chicagon University of Chicago Press.

Haslett, R. $S_{*}$ Duvall Young, $J_{,}$\& McGalliard, J. N. (1994). Tratmatic retinal angiopathy and seat belts: Pathogenesis of whiplash injury. Eye, 8(6),615-617.

Hayes, R. L., Lyeh, B. G., \& Jenkins, L. W. (1989). Neurochemical mechanisms of milid and moderate head injury: implications for treatment. In H. S. Levin, H. M. Eisenberg, \& A. L. Benton (Eds.), Mild Head Injwry, (pp, 54-79). New York: Oxford University Press.

Henty, G. K. (1994). DSM-IV: Proposed criteria for postconcussive disonder. Journat of Newropsychicury and Clinical Neurosciences, $6(1), 58$.

Heyman, A., Wilkinson, W. E., Staftord, J. A., Helms, M. J., Sigmon, A. H., \& Weinberg, T. (1984). Alzheimer's disease: A study of epidemiological aspects. Annals of Menrology, 15(4), 335-34l.

Hirsch, S. A. Hirsch, P. J., Hiramoto, H., \& Weiss, A. (1988). Whiplash syndrome, Fact or fiction? Orhopedic Climics of North America, 19(4), 79:-795.

Hof, P. R. Bouras, C., Buee, L., Delacourte, A., Perl, D. P., \& Morrison, J. H. (1992). Differential distribution of netrofibrillary angles in the cerebral conex of tementia pugulistica and Alzheimer's disease cases. Acta Nearopathologica. $85(1), 23-30$.

Hofman. D. A. (1994). Sublypes for postconcussional disorder. Sournal of Neuropsychatiy and Chical Nemrosciences, $6(3), 332$.

Hofman, A. Schulte, W., Tanja, T. A., Van Duin, C. M., Hasxma, R. Lameris, A. J." Otten, V. M., \& Sann, R. J. (1989). History of dementia and Parkinson's disease in first-degree relatives of pallonts wih Altheimen's disease. Neurology, $39(12), 1589-1592$.

Houx, P. J, \& Jolles, J. (1994). Vulnerability factors for age-related cognitive decline. In R. L. Isadeson \& $\mathrm{K}$. 1F. Jensen (Eds.). The Vulnerable Brain and Emvironwental Risks, (Vol, 3, pp. 25-41). New York: Plemum Press.

Houx, P. J., Vreeling, F. W. \& Jolles, J. (1991). Rigorous health screening reduces age effect on memory scanning task. Brain and Cognition, 15(2), 246-260.

Hugenholu, H., Suss, D. T., Stethem, L. L., \& Richard, M. T. (1988). How long does it take to recower from a mild concussion? Neurosurgery, 22(5), 853-858.

Huppert, $\mathbb{F}$. A., \& Brayne, C. (1994). What is the relationship between dementian and nomal aging? lla F. A.. Huppert, C. Brayne, D. W. O'Connor (Eds.). Dementa and Nomal Aging. (pp. 3-11). New York: Cambridge University Press.

Jacobson, R. R. (1995). The post-concussional syndrome: Physiogenesis psychogenesis and malingering. An integrative model. Joumal of $P_{\text {sychosomatic Researd }}, 39(6), 675-693$.

Jagger, J., Vemberg, K., \& Jane, J. A. (1987). Air bags: Reducing the toll of brain trauma. Neurosurgery, 20(5), $815-817$ 
Jakobsen, I., Baadsgard, S. E. Thomsen, S., \& Henriksen, P. B. (1987). Prediction of post-concussional sequelate by reaction time test. Acta Neurologica Scandinavica, 75(5), 341-345.

Jane, I. A., \$teward, O., Gennarelli, T. A. (1985). Axonal degeneration indeced by experimental noninvasive minor head injury. Mournol of Neurosurgery, $62(1), 96-100$.

Jeffreys, E. T. McSweeney, T., \& Park, W. M. (1980). Disorders of the Cervical Spine London: Butterworths.

Jensen, C. (1979). Leaning performance in mice selected for brain weight. In M. E. Hahn, C. Jensen, \& B. C Dudek (Eds.), Development and Evolution of Brain Size, (pp. 201-224). San Diego, CA: Academic Press.

Jolles, J., Houx, P. I. Vreeling, F. W., Bohnen, N. I., \& Reyersen van Buruen, E. J. (1990). Biological risk factors for accelerated cognitive aging and denwentia: findings from a multiple cohort study. Newrobiology of Aging, $/ /(3), 293$.

Jorm, A. F., Korten, A. E., \& Henderson, A. S. (1987). The prevalence of dementia: A quantitative integration of the literature. Acta Psychiatrica Scandincivica, $76(5), 465-479$.

Katzman, R., Terry, R, DeTeresa, R, Brown, T., Davies, P., Fuld, P., Renbing, X, \& Peck, A. (1988). Clinical, pathological, and neurochemical changes in dementia: A subgroup with preserved mental status and numerous neocortical placjues. Annals of Neurology, 23(2), 138-144.

Kewman, D. G., Yanus, B., \& Kirsch, N. (1988). Assessment of distractibility in auditory comprehension after traumatic brain injury. Brain Injury, 2(2), 131-137.

Kischka, U., Etlin, T. M., Heim, S., \& Schmid, G. (1991). Cerebral symptoms following whiplash injury. European Neurology, 3/(3), 136-140.

Klein, M. Houx, P. J.x Jolles, J. (1996). Long-term Persisting Cognitive Sequelae of Traumatic Brain Injury and the Effect of Age. Joumal of Nerwous and Mental Disease, 184(8), 459-467.

Kokmen, E, Chandra, $V_{\text {on }}$ \& Schoenberg, B. S. (1988). Trends in incidence of dementing illness in Rochester, Minnesota, in three quinquennial periods, 1960-1974. Neurology, 38(6), 975-980.

Kolb, B., \& Whishaw, I. Q. (1990). Principles of cerebral asymmetry. In B. Kolb \& I. Q. Whishaw (Eds.), Fundamentals of Human Neuropsychology, (3rd ed., pp. 347-382). New Yotk: W.H. Freeman and Company.

Kondo, K., \& Yamashita, II. (1990). A case-control study of Alzheimer"s disease in Japan: Association with inactive psychosocial behaviors. In K. Hasegawa \& A. Homma (Eds.), Psychogeriarrics, Biomedical and Social Advances, (pp. 49-53). Amsterdam, The Netherlands: Excerpta Medica:

Kraus, J. F., Black, M. A., Hessol, N., Ley, P., Rokaw, W., Sullivan, C., Bowers, S.; Knowlton, S., \& Marshall, L. (1984). The incidence of acute brain injury and serious impairment in a defined population. American Joumal of Epideniology, 119(2), 186-201.

Kraus $_{\|}$J. F., \& Nourjah, P. (1988). The epidemiology of mild uncompllicated head injury. Jourmal of Trauma, $28,1637-1643$.

Kraus, J. F. \& Nourjah, P. (1989). The epidemiology of mild head injury. In H. M. E. H.S. Levin, A.L. Bienton (Ed.), Mild Head Injury" (pp. 8-22). New York, NY, US: Oxford University Press.

Leininger, B. E, Gramling, S. E., Farrell, A. D., Kreutzer, J. S., \& Peck, E. (1990). Neuropsychological deficits in symptonatic minor head injury patients after concussion and mild concussion. Journal of Nearology, Nenosostergery, and Psychiarry, 53(4), $293-296$.

Levin, H., \& Kraus, M. F. (1994). The frontal lobes and traumatic brain injury. Special Issue: The frontal lobes and metropsychiatric illness. Journal of Neuropsychiary and Clinical Neurosciences, 6(4), 443-454.

Lovin, H. S., Amparo, E. G., Eisenberg, H. M., Williams, D. H., High, W. M., McArdle, C. B., \& Weiner, R. L. (1987a). Magnetic resonance imaging and computerized comography in relation to the neurobehavioral sequele of nild and moderate head injuries. Journal of Newrosurgery, 66(5), 706-713.

Levin. H. S., Eisenberg, H. M. \& Benton, A. L. (1989). Mild Head Injury. New York: Oxford Uniwersity Press.

Levin, H. S., Gary, H. E., Jr., Eisenberg, H. M., Ruff, R. M., Bardh, J. T., Kreutzer, J., High, W.. M., Jr., Portman, S., Foulkes, M. A.. Jane, J. A., Marmarou, A. \& Marshall, L. F. (1990). Neurobehavioral outcome I year after severe head injury: Experience of the Traumatic Coma Data Bank. Journal of Neurosurgery, 73(5), 699-709.

Levin, H. S., High, W. M., Goldstein, F. C., \& Williams, D. H. (1988). Sustained attention and information processing speed in chtonic survivors of severe closed head injury. Scandinavian Joumal of Rehabilitation Medicine, $17,33-40$.

Levin, H. S., Mattis, S., Ruff, R. M., Eisenberg, H. M., Marshall, L. F., Tabaddor, K., High, W. M., \& Frankowski, R. F. (1987b). Neurobehavioral outcome following minor head injury: A three-center study. Joumal of Neurosurgery, 66(2), 234-243.

Lezak, M. D. (1983). Batteries and composite tests for brain damage. In M. D. Lezak (Ed.), Neuropsychological assesssment, (2nd ed.. pp. 560-575). New York: Oxford University Press. 
Lezak, M. D. (1993). Newer contributions to the neuropsychological assessment of executive funthons. Nownul of Head Trawna Rehabilitation, 8(1), 2431 .

Lishnian, W. A. (1988). Physiogenesis and psychogenesis in the "postwoncussional syndrome". Brith Jourwal of Psychiatry. $153(460), 460469$.

Loken, W. J."Thornton, A. E, Otio, R. L, \& Long, C. J. (1995). Sustained attention after sevene bosed head injury. Neuropsychology, $9(4), 592-598$.

Luck, S. d, Heime, H. I., Mangun, G. R., \& Hillyard, S. A. (1990). Visual exent-related potentials index focused attention within bilateral stimulus arrays. IL. Functional dissociations of $\mathrm{Pl}$ and N1 components. Electroencephalography and Clanical Netrophysiology, 75(6), 528-542.

Mac Nab, $J$ (1982). Acceleration extension injuries of the cervical spine In R. H. Rothman d F. A. Simcone (Eds.). The Spine, (2nd ed., pp. 647-660). Philatelphia: WB Saunders.

MacFlyn, G., Montgomery, E. A., Fenton, G. W., \& Rutherford, W: (1984). Measurement of reaction time following minor head injury. Jounal of Newrology, Neurosurgery, and Psychiatry, 47(12), 1326-1331.

Mahon D., \& Elger, C. (1989). Analysis of postraumatic syndrome following a mild head injury. Jountal of Neuroscience Nursing, 21(6), 382-384.

Marshall, L. F, \& Ruff, R. M. (1989). Neurosurgeon as victm. In H. S. Levim, H. M. Eisenberg. d A. L. Benton (Eds.). Mild head injury, (pp. 276-280). New York: Oxford University Press.

Martand, H. S. (1928). Punch drunk. Joumal of the American Medical Association, 91, 1103-1107.

Mathew, P., Graham, D. I., Bullock, R., Maxwell, W.., McCulloch, 1., Re Teardale, G. (1994). Focal brain injury: Histological evidence of delayed inflammatory response in a new rodent model of focal cortical injury. Acta Newrochinurgica-Supplementum, 60, 428-430.

Matsuyama, H., \& Nakamura, S. 1978). Senile changess in the brain in the Japanese: Incidence of Alaheimer's neurofibrillary change and senile plaques. Aging (New York), 7, 287-297.

Mayes, C. D., Pelco, L. E., \& Campbell; C. J. (1989). Relationship among pro- and post-injury intelligence, length of coma and age in individuals with severe closed head injury. Brain Injwry, 3(3), 301-313.

Mayeux, R., Otman, R. Maestre, G, Ngai, C., Tang, M. X., Ginsberg, H., Chun, M., Tycko, B., \& Shelanski, M. (1995). Symergistic effects of tramatic head injury and apolipoprotein-epsilon 4 in patients with: Alzheimer's disease. Newrology, 45(3), 555-557.

Mazzucchi, A., Cattelani, R., Missale, G.; Gugliotta, M., Brianti, R. \& Parna, M. (1992). Head-injtured subjects aged over 50 years: correlations between wariables of trauna and neuropsychological follow-up. Joumal of Neurology, 239(5), 256-260.

McAllister, T. W. (1992). Neuropsychiatric sequelae of head injuries. Psychiaric Clmics of North America, $15(2), 395-413$.

McClelland, R. J., Fenton, G. W., \& Rutherford, W. (1994). The post concussional syndrome revisited Joumal of Research in Sacial Medicine, 87(9), 508-510.

MeDowd, J. M. \& Birren, J. E. (1990). Aging and attemionall processes. Ir J. E. Birren \& K. W. Schate (Eds.), Hawdbook of the Psychology of Aging, (pp. 222-233). San Diego. Academic Press.

McKinlay, W. W., Brooks, D. N., Bond, M. R., Martinage, D. P., \& Marstall, M. M. (1981). The short-1erm outcome of severe blunt head injury as reported by refatives of the imjured persons. Jowmal of Newrology. Neurosurgery, and Psychatry, 44(6), 527.

McLean, A., Temkin, N. R., Dikmen, S., \& Wyler, A. R. (1983). The behavioral sequelae of head injury. Joumal of Clinical Neuropsychology, $5(4), 361 \times 376$.

MoMillam, T. M. \& Glucksman, E. E. (1987). The neuropsychollogy of moderate head injury, Joumal of Neurology, Neurosurgery, and Psychiatry, 50(4), 393-307.

Meltzer, H. Y. \& Lowy, M. T. (1987). The serotonin hypothesis of depression. In H. Y. Meltzen (Ed.), Psychopharmacology: The Third Generation of Progress, (pp. 513-526). New York: Raven Press.

Mesulan, M. M. (1981). A cortical network for clirected attention and tumilateral neglect. Amnals of Neurology, $10(4), 309.325$.

Middelboe, T. Andersen, H. S., Birkil-Smith, M., \& Eviis, M. L. (1992). Minor head injury: impact on general health after 1 year. A prospective follow-up study. Acta Nearologica Scandinawica, 85(1), 5.9.

Middelboe, T., Birket Smith, M., Andersen, H. S., \& Fris, M. L. (1992). Personality traits in patients with postconcussional sequelae. Journal of Personality Disorders, 6(3), 246-25.5.

Miller, E (1970). Simple and choice reaction time following sewere head injury. Contex, 6(1), $121-127$.

Miller, $\mathbb{F}$. W., Hicks, S. P., D'Amato, C. S., \& Landis, J. R. (1984). A descriptive study of neuritic plaques and neurofibrillary tangles in an autopsy population. American Journal of Epidemiology, 120,331-341.

Miller J. D. (1993). Head injury. Joumal of Neurology, Neurosurgery and Psychiatry, 56(5), $440-44.7$. 


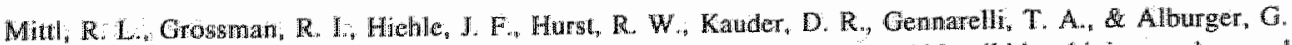
W. (1994). Frevalence of MR evidence of diffuse axonal injury in patients with mild head injury and normal

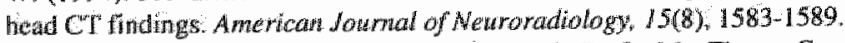

Monigomery, E. A, Fenton, G. W. McClelland, R. J., MacFlyn, G. \& Rutherford, W. H. (1991) The peychobiolwgy of minon head injury. Psychological Medicine, $21(2), 375384$

Morris, R. G. \&opelman, M. D. (1986) The memory deficits An Aldimertype dementia A review.

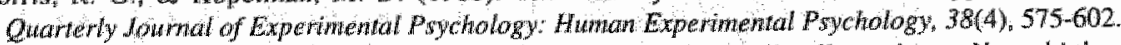

Morrison, I. H. (1993). Differentiat wulnerability, connectivity, and cell typology Newrobiology of Aging, $14(1), 51.54$.

Mortiner, I. A. (1994). What are the risk factors for dementia? In F. A. Huppert, C. Brayne, \& D. W. O Connor (Eds.), Dementa and Normal Aging. (pp. 208-243). New York Cambridge University Press.

Mortimer, J: A, French, L. R, Hution, J. T. \& Schumar, L. M. (1985) Head tranma as a risk factor for Althenmers disease. Newology, 35(2), 264-267.

Mortimer, J. A., French, L. R.; Hutwon, J. T., Schuman, L. M. Christians, B: \& Boatman, R. A. (1983). Reported head trama in an epidemiologic study of Alzhemer's disease. Neurology, 33( Suppl. 2), 85.

Mortimer, J. A., Pe Pirozzolo, F. J. (1985). Remote effects of head trauma. Developmental Neuropsychalogy. $1(3), 215.229$

Mortimer, I. A., van Duifm, C. M., Jorm, A., Soinimen, H, Fratighioni, L., Kondo, K., Hofman, A. Shalat, S. L., Chandra, V., Heymar, A., Kokmen, E. \& Graves, A. B. (1991). Head trama as a risk factor for Alzheiner disease. A colliborative re-analysis of caseucontrol studies: Imtemational Joumal of Epidemiotogy. $20($ Suppl. 2$), 28 \cdot 35$.

Motter, B. C. (1993). Focal attention produces spatidly selective processing in visual cortical areas $\mathrm{V} 1, \mathrm{~V} 2$, and V4 in the presence of competing stimuli: Joumal of Neurophystology, $70(3), 909.919$.

Nawashire, H, Shima, K., \& Chigasaki, H. (1994), Blood-brain barrier, cerebral blood tlow, and cerebral plasma volume immediately aftor head injury in the rat. Acta Neurockirngica-Supplenewtum, $60,440-442$.

Nabes, R. D. (1989). Semantic menory in Alzheimer's disease. Psychological Bulletin, 106 (3); $377-394$.

Nebes, R. D., \& Brady, C. B. (1989). Focused and divided attention in Altheimer's disease. Cortex, 25(2), 305 315.

Nee, L. E., Polinsky, R. J., Eldridge, R., Weingartner, H., Smallberg, S. \& Ebert, M. (1983). A family with lwistologically confimed Alzeimer's disease. Archives of Neurology, 40(4), 203-208.

Nevin, $N_{*} \mathrm{C} .(1967)$. Neuropathological changes in the white matter following head injury. Journal of Newropathology and Experimental Newrology $26(1), 77-84$.

Newcombe, F, Rabbitt, P., \& Briggs, M. (1994). Minor hend injury: Pathophysiological or iatrogenic sequelae? Jowmal of Newrology, Newrogurgen, and Psychiatry, 57(6), 709-716.

Newell, A., \& Simon, H. A. (1972). Human Problem Solving. Engelwood Cliffs, NJ: Prentice Hall.

Nicoll, J. A. Roberts, G. W., \& Graham, D. I. (1995). Apolipoprotein $\mathbb{E}$ epsilon 4 allete is associated with deposition of enmyloid beta-protein following head injury. Nature and Medhome, $8(2), 135-137$.

Noman, D. A. \& Shallice, T, (1986). Attention to action: Willed and automatic control of behavior. Ir R. J. Davidson, G. E. Schwart, \& D. Shapiro (Eds.), Conseiowshess and Self-Regntation, (pp. 1-18). New York: Plenum Press.

Nortis, S. H, \& Watt, I. (1983), The prognosis of nock injuries resulting from rear-end vehicle collisions. Jompul of bone and Joint Surgery, 65B(5), 608-611.

OShaughnessy, E. I., Fowler, R. S., \& Reid, V. (1984). Sequelas of nild closed head inguries. Journal of Family Practice, $18(3), 391-394$.

Oddy, M., Coughan, T., Tyerman, A., \& Jenkins, D, (1985). Social adjustment after closed-head injury: A further follow up sever years after injury. Jounal of Newology, Newrowugery, and Psychiatry, 48(6), 564568.

Onnuya, A. K., Fass, F., \& Yarnell, P. (1968). Whiplash injury and brain damage. Journat of the American. Mecical Association, 204(4), 285289

Ommaya, A. K., \& Gennatelli. T. A. (1974). Cerebral concussion and traumatic unconsciousness: Correlation of expermental and clinbal observations in blunt lyead injuries. Brait, 97(4), 633-654.

Oppenheimer, D.R. (1968). Microscopic lesions in the brain following head injury. Journal of Neurology. Newrosurgery, and Psychaty, 3(4), 299-306.

Ortiz. T. Martin Loeches" M., \& Vila, E. (1990). Frontal lobes and ageing effect on the P300 component of the anditory event-related potentials. Applied Psychology: An International Review; 39(3), 323-330.

Ot, L., MoClain, C. J., Gillespie, M., \&oung, B. (1994). Cytokimes and metabolic dysfunction after severe thead injury. Jownal of Neurotramma, $/ /(5), 447-472$. 
Parasuraman; R. (1984). Sustaned attention in derection and discrimination. In R. Parasuraman 1 D. R. Davies

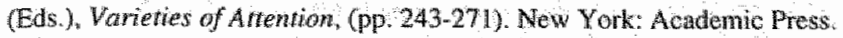

Parasuraman; R. Greenwood, P.M., Haxby, I. V., Grady, C. L. (1992). Visuospatial atlention in dementia of the Alwemer type. Brain, 1/5(3), $711-733$.

Parasuraman, R., Haxby, J, V. (1993). Attertion and brain function in Alaheimets disense: A review, Meuropsychology, $7(3), 242-272$

Parasuraman, R., Mutter, S. A., \& Moloy, R. (1991). Sustaned attention following mild closed head injury. Jownal of Clinical and Experimental Newropsychology, $13(5), 789,811$.

Parasuraman;, R., Warm, J. S., \& Dember, W. N. (1987). Vigilamce: Taxonomy and utility. In L. Mark, J. S. Warm, \& R. L. Huston (Eds.). Ergonomics and Human Factors: Recent Restarch, (pp. 11-32). New York: Springer-Verlag.

Pardo, J. V., Pardo, P. J., Janer, K. W., Raichle. M. E. (1990). The anterior cingulate cortex mediates processing selection in the Stroop attentional conflict paradigm. Procedings of the National Academy of Science, $87(1), 256-259$.

Paschalis, C., Polychronopoulos, P., Lekka, N. P., Harrison . M. I. \& et al (1990). The role of head injury. surgical anaesthesia and family history as aetological foctors in dementia of Alaheimer type: A prospective study. Dementia, $1(1), 52,55$.

peters, A., Leahu, D. Moss, M. B. \& McNally, K. J. (1994). The effects of aging on area 46 of the frontal cortex of the rhesus monkey. Cerebral Correx, 4(6), 621-635.

Petersen, S. E., Fox, P. T. Posner, M. 1. Mintun, M., \& Raichle, M. E. (1988). Positron emission tomography studies of the cortical anatomy of single-word processing. Nature, 337(6157), 585-589.

petersen, S. E., Fox, P. T., Posner, M. I. Mintun, M. A., \& Raichle, M. E. (1989). Positron emission tomography studies of the processing of single words. Journal of Cognitwe Newroscience, $1(2), 153-170$.

Ponsford, J." Kinsella, G. (1992). Attentional deficis following closed head injury. Joumal of Clinical and Experinental Neuropsychology, $14(5), 822-838$.

Posner, M. I. (1988). Structures and functions of selective attention. In T. Boll \& B. Bryant (Eds.), Master Lectures in Clinical Newropsychology, (pp. 171-202). Washington, DC: American Psychological Assuciation.

Posner, M. I., Nissen, M., \& Odgen, W. C. (1978). Attended and unattended processing modes: The role of set for spatial localization. In H. L. Pick \& E. Salman (Eds.), Modes of Percening and Processing Information, (pp. 137-157): Hillsdale, NJ: Lawrence Erlbaum Associates.

Posner, M. I. Petersen, S. E. Fox, P. T., \& Raichle, M. E. (1988). Localization of cognitive operations in the louman brain. Science, 240(4859), 1627.1631 .

Powlishock, J. T., Becker, D. P., Cheng, C. L. Y., \& Vaughan, G. W. (1983). Axonal change in minor brain injury, Jounal of Newropathology and Experimental Neurology, 42(3), 225.

Powlishock, J. T., \& Cobum, T. H. (1989). Morphopathological change associatted with mild head injury. In H. S. Levin, H. M. Eisenberg. \& A. L. Benton (Eds.). Mild Hedad Injury, (pp. 37-53). New York: Oxford Universily Press.

Powlishock, J. T., \& Jenkins, L. W. (1995). Are the pathobiological changes evoked by traumatic brain imjury immediate and irreversible? Brain Pathology, 5(4), 415-426.

Price, I. L, Davis, P. B., Marris, J. C. White, D. I. (199!). The distribution of tangles, plaques and related immunohistochemical markers in healthy aging and Alwhimer's disease. Nearobiology of Agimg, $12(4), 295-$ 312

Quinn, N. P. Rossor, M. N., \& Marsden, C. D. (1986). Dementia and Parkinson's disease-pathological and neurochemicall considerations. British Medical Joumal, $42(1), 86,90)$.

Radanow, B. P., Di Stefano, G. Schnidrig, A. \& Ballinari, P. (1991). Role of psychosocial stross in recowery from cormon whiplash. Lamcet, $338(8769)$. 712-715.

Radanov, B. P., Dvorak, J, \& Valach, L. (1992). Cognitive deficits in patients after soft tissue injury of the cerwical spine. Spine, $17(2), 127-131$.

Radanov, B. P., Sturzenegger, M., De Stefano, G., \& Schnidrig, A. (1994). Relationship between early somatic, radiological, cognitive and psychosocial findings and outcome during a one-year follow-up in 17 patients suffering from common whiplash. British Jownal of Rhewmatology, 33(5), 442-428.

Radanow, B. P., Sturzenegger, M., \& Di Stefano, G. (1995). Long-term outcome alter whiplash injury: A 2*year follow-up considering features of injury mechanism and somatic, radiologic and psychosocial findingrs. Nedicine, $74(5), 281-297$.

Raichle. M. E., Fiez, J. A., Videen, T. O, Macleod, A. M. K., Pardo, J. V., Fox, P. T., \& Peterser, S. E. (1994). Practice-related changes in human brain functional anatomy during nonmotor learnirg. Cerebrat Cortex, $4(1), 8-26$ 
Randolf-Swari. J. Lesser, I. M., Boone, K. B. Miller, B. L., \& Mena, I. (1995). Cerebral blood fow changes in nonal anging. SPECT measurements. Inemational Jowmal of Geriatric Psychiany, 10(6), 437-446.

Ratsmusson, D. X., Brand, J., Marin. D. B, \& Folstein, M. F. (1995). Head injury as a risk factor in Alweimer"s disease. Brain lnjury, $9(3), 213-219$

Reitan, R. M. Wolfson, D. (1985). The Halstead-Reitan Neuropsychological Test Banery: Theory and Clinical Miterpretation. Tuczon; $A Z_{\text {i }}$ Neuropsychology Press.

Reitan, R. M., Wolfon, D. (1989). The Seashore Rhythm Test and bram functions. The Clnical Neuropsychologist, $3(1), 70-78$.

Rimel, R: W. Giordan, B., Barth, J. T., Bal, J. T., \& Jane, J, A. (1981). Disability caused by nuinor head Jajury, Neurosurgery, $9(3), 221-228$.

koberts, G. W. Alsop, D. \& Bruton, C. J. (1990). The occult aftermath of boxing. Journal of Newrology, Neurosurgery, and Psychiatry, $53(5), 373-378$.

Roberts, O. W. Genlleman, S. M., Lynch, A., Graham, D. I. (1991). Beta A4 amyloid protein deposition in brain after hewd trawna Lancet, $\$ 38(8780), 1422-1423$.

Roberts, G. W., Gentleman, S. M., Lynch, A., Murray, L., Landon, M., Graham, D. I. (1994). B Amyloid protein deposition in the brain after severe head injury: Implications for the pathogenesis of Alzheimer's disease. Joumal of Neurology, Newrosurgery, and Psychionry. 57(4), 419.425.

Rogers, R. D. \& Monsell, S. (1995). Costs of a predictable switch between simple cognitive tasks. Journal of Etperimestal Psychology: General, 124,207-221.

Roses, A. D. (1994). Apolipoptotein $\mathrm{E}$ affects the rate of Alzheimer's disease expression: Beta-amyloid burden is. a secondary conseguence dependent on A.POE genotype and duration of disease. Joumal of Neuropathology and Experimental Neurology, $53(5), 429-437$

Rosner, M. J., Newsome, H. H., \& Becker, D. P. (1984). Mechanical brain injury: The sympathoadrenal rasponse. Joumal of Neurosurgery, 61(1), 76-86.

Roth, M. (1986). The association of clinical and neurological findings and its bearings on the classification and atiology of Alaheiner's disease. British Medical Bulletin, 42(1), 42-50.

Rubin, E. H. Morris, J. C. Grant, E. A., Vendegna, T. (1989). Very mild senile denentia of the Alzheimer type: I. Clinical assessment. Archives of Neurology, 46(4), 379-382.

Rudelli, R., Strom, J. O., Welch, P. T., \& Ambler, M. W. (1982). Posttraumatic premature Alzheimer"s disease. Netropathologic findings and pathogenetic considerations. Archives of Neurology, 39(9), 570w575.

Ruff, R. M., Crolich, J. A., Troster, A. I., Marshall, L. F.., Buchsbaum, M. S., Lottenberg, S., \& Somers, L. M. (1994). Selected cases of poor outcome following a minor brain trauma: Comparing neuropsychological and positron emission tomography assessment. Brain Injury, 8(4), 297-308.

Ruff, R. M., Levin, H. S., Mattis, S., High, W. M., Marshall, L. F., Eisenberg, H. M., \& Tabaddor, K. (1989). Recovery of memory after mild head injury: A three-center study. In H. S. Levin, H. M. Eisenberg, \& A. L. Benton (Eds.), Mild Fead hijary, (pp. 176-188). New York: Oxford University Press:

Rugg, M. D., Cowan, C. P., Nagy, M. E., Milner, A. D., Jacobson, I., \& Brooks, D. N. (1989). CNV abnormalitios following closed head injury. Brain, $1 / 2(2), 489-506$.

Rutherford. W. H. (1989). Postconcussion symptoms: Relationship to acute neurological indices, individual differences, and circumstances of injury. In H. S. Levir. H. M. Eisenberg, \& A. L. Benton (Eds.), Mild Head Injury, (pp. 217-228). New York: Oxfond Universilty Press.

Rutherford, W. H., Merrett, J. D. \& MoDonald, J. R. (1979). Symptoms at one year following concussion from minor lowad injuries. Injury, $10(3), 225-230$

Satz, P. (1993). Brain reserve capacity on symptom onset after brain injury: A formulation and review of evidence for threshold theory. Neuropsyehology, $7(3), 273-295$.

Sbordone, R. $J_{,}$\& Liter, J. C. (1995). Mild traumatic brain injury does not produce post-tranmatic stress disorder. Brain Lwiny, $9(4), 405-412$.

Sicheibel, A. B. (1980). Anatomical and physiological substrates of arousal: A view from the bridge. In I. A. Hobson \& M. A. B. Brazier (Gds), The Rericalar Fomation Revisited, (pp. 55-66). New York: Raven Press.

Schmidt, R. HA., Grady, M. S. (1995). Loss of forebrain cholinergic neurons following fluid-percussion injury: Implications for cognitive impaiment in closed head injury. Jowrol of Neurosurgery, 83(3), 496-502.

Schneider, W., Shiffrin, R. M. (1977). Controlled and automatic human information processing: I. Detection, search, and attention. Psychological Review, 84(1), 1-66.

Shalat, S. L.., Seltzer, B., Pidcock, C., Baker, E. L. (1987). Risk factors for Alzheimer's disease: A casecontrol study. Neurology, $37(10), 1630-1633$.

Shallice, T. (1982). Specific impairments of planning. In D. E. Broadbent \& L. Weiskrantz (Eds.. The Neuropsychology of Cogmitive Function, (pp. 199-209). London: The Royal Society. 
Shallice. T. (1988): From Neuropsychology to Mental Structure. New York: Cambridge University Piess.

Shallice, T. (1994). Multiple levels of control processes. In C. Unitha \& M. Moscovitch (Eds.) Antertion and Perfornance: Conscions and Nonconscions Information Processing, (Vol. XV, pp. 395-420). Cambridge, MA: MIT Press.

Shallice, T. \&urgess, P. W. (1991a). Deficits in strategy applications following frontal lobe damage in man. Brain, $114(2), 727-741$.

Shallice, T. \& Burgess, P. W. (1991b). Higher-order cognitive impairments and frontal lobe lesions in man. In H. S. Levin, H. M. Eisenberg, \& A. L. Benton (Eds.), Frontal Lobe Function and Injury. (pp. 125-138). New York: Oxford University Press.

Shiffrim, R. M., \& Schneider, W. (1977). Controlled and automatic human information processing: II. Perceptual learning, automatic attending and a general theory. Psychological Review, 84(2), 127-190.

Silver, J. M., Yodofsky, S. C., \& Hales, R. E. (1991). Depression in traumatic brain injury. Neuropsychiatry. Neuropsychology, and Behavioral Neurology, 4(1), 12-23.

Soininen, H., \& Heinonen, O. P. (1982). Clinical and etiological aspects of senile dementia. European Neurology, 21(6), 401-410.

Spitzer, H. R., Desimone, R., \& Moran, J. (1988). Increased attention enhances both behavioral and neuronal performance. Science, $240(4850), 338-340$.

Stablum, F., Leonardi, G., Mazzoldi, M.. Umilta, C., \& Morra, S. (1994). Attention and control deficits following closed head injury. Correx, 30(4), 603-618.

Steptoe, A. (1991). Invited review. The links between stress and illness. Joumal of Psychosomatic Research $35(6), 633-644$.

Stern, Y. Alexander, G., Prohovnik, I, \& Mayeux, R. (1991). Education provides a cognitive reserwe against the clinical manifestations of Alzheimer"s disease: Evidence from regiomal cerebral blood flow. Society for Neuroscience Abstracts, 17, 1258 .

Storandt, M., \& Hill, R. D. (1989). Very mild senile dementia of the Alzheimer type: II. Psychometric test performance. Archives of Neurology, 46(4), 383-386.

Stroop, J. R. (1935). Studies of interference in serial werbal reactions. Joumal of Experimental Psychology, 18(6), 643-662.

Stuss, D. T. \& Benson, D. F. (1984). Neuropsychological studies of the frontal lobes. Psychological Bulletin, $95(1), 3-28$.

Stuss, D. T., Ely, P., Hugenholtz, H., Richard, M. T., LaRochelle, S., Poirier, C. A., \& Bell, I. (1985). Subtle neuropsychological deficits in patients with good recovery after closed head injury. Neurosurgery, $17(1), 41$ 47.

Stuss, D. T., Sitethem, L. L., Hugenholtz, H., Picton, T., Pivik, J., \& Richard, M. T. (1989). Reaction time after head injury: Fatigue, divided and focused attention, and consistency of performance Joumal of Neurology. Newrosurgery, and Psychiatry, 52(6), 742-748.

Sulkava, R., Erkinjuntti, R., \&alo, J. (1985). Head injuries in Alzheimer's disease and vasculat dementia. Neurology: 35(12), 1804 .

Symonds, C. (1962). Concussion and its sequelae. Lancet, I, 1-5.

Taylor, S. F., Kornblum, S., Minoshima, S, Oliver, L. M., \& Koeppe, R. A. (1994). Changes in medial cortical blood flow with at stimulus-response compatibility task. Neuropsychologia, 32(2), 249*255.

Teasdale, G. \&ennett, B. (1974). Assessment of coma and impaired consciousness: A practical scale. The Lancet, 2(872), 81-83.

Tomlinson, B. E., Blessed, G., \& Roth, M. (1970). Observations on the brains of demented old peopie. Joumal of the Neurological Sciences, 1/(3), 205-242.

Towbin, A. (1987). Neuropathologic correlates. In D. E. Tupper (Ed.), Soft Neurological Signs, (pp. 157-178). New York: Grune \& Stratton.

Turk, D. C., \& Rudy, T. E. (1991). Cognitiwe factors and persistent pain; A glimpse with Pandora's box. Cognitive Therapy and Resiearch, 16,99.122.

Umilia, C. A., Nicoletti, R., Simion, F., Tagliabue, M. E., B Bagnara, S. (1992). The cosit of a stratcgy. European Journal of Cognitive Psychology, 4(1), 21-40.

Vakil, E., Weisz, H., Jedwab, L., Grosswasser, Z., \& Aberbuch, S. (1995). Stroop Color-Word Task as a measure of selective attention: Efficiency in closed-head-injured patients. Joumal of Clinical and Experimental Nestropsychology. 17(3), 335-342.

Van den Broek, M. D. Bradshaw, C. M. \& Szabadi, E. (1993). Utility of the Modiffed Wisconsin Card Sorting Test in meuropsychological assessment. British Jownal of Clinical Psychology, 32(3), 333-343. 


\section{CHAPTER 4}

Van Wolfoldar P. C., Brovwer, W. H. Wam Zomeren, H. H (1990), Driving ability 5 to 10 years after severe

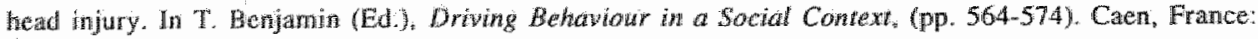
Paradignte.

Van Zomeren, A. H. (1981). Reaction Thine and Anention after Closed-Head Injury. Lisse, The Netherlands: Swets Publishing Services.

Wan Zomeren, A. H., Brouwer W. H. (1987). Head injury and concepts of altention. In H. S. Levin, J.

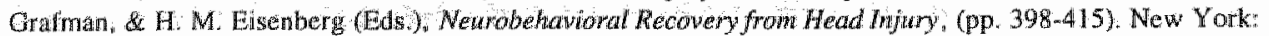
Oxford Uniwergity Press.

Van Zomeren, A. H., Brouwer, W. H. (1994). Closed head injury, Clinical newropsyohology of attention, (pp. 63-94): New York: Oxford University Press:

Van Zomeren, A. H. \& Deilman, B. C. (1976). Diferential effects of simple and choice reaction after closed head injury, Chinical Newrology and Nenrogsurgery, $79(1,64-70$.

Van Zomeren, A. H. d Deelman, B. G. (1978). Long-tern recovery of visual reaction time after closed head injury. Journat of Newology. Newrosurgery, and Psychiarry, 4J(1), 452-457.

Vut Zontern, A. H. \& Van den Burg, W. (1985). Residual complaints of patients two years after severe head injury. Nowrnal of Newrology. Neurasurgery, and Psychatry, 48(1), $21-28$

Varney, N. R., Bushnel, D. L., Nathas, M. Kalun, D., Roberts, R, Rezai, K., Walker, W., \& Kirchner, P. (1995). NeuroSPECT correlates of disabling mild head injury: Preliminary findings. Jounal of Head Trauma Rehabilitarion, $10(3), 18-28$.

Waddel, P. A., Q Gronwall, D. M. (1984). Sensitivity to bight and sound following minor head injury. Acra Nentologica Sicandinatica, 69(5), 270-276.

Walst, K. W. (1987), Neuropsychology: A Clinical Approach. New York. Livingston.

Wiberger, $J, E$ (1993) Minor head injuries in American lootball. Prevention of long term sequelae. Sports Madicime, $15(5), 338 \cdot 343$.

Wilkins, A. I., Shallice, T. \& McCarty, R. (1987). Frontal lesions and sustained attention. Newropsychologia, $25(2), 359-365$.

Willerman, L., Schultz, R., Rutledge, J. N, \& Bigler, E. D. (1991). In vivo brain size and intelligence. Intelligence. $15(2), 223-228$.

Willerman, L., Schultz, R., Rutledge, J. N. \& Bigler, E. D. (1992). Hemisphere size asymmetry predicts relative verbal and nonverbal intelligence differently in the sexes: An MRI study of structure-function relations, Futelligence, $16(3-4), 315-328$.

Wrightson, P., \& Gronwalt, D. (1981). Time off work and symptoms after nurnor head injury, Injwy, 12(6), 445-454.

Yarmell, P. R., \& Rossie, G. V. (1988). Minor whiplash head injury with major debilitation. Brain Injury, 2(3), 255-258.

Yokota, H., Kunokawa. A.. Otsukn, "T", Kobayashi, S., \& Nakazawa, S. (1991). Significanco of magnetic resonance imaging in ancute head injury. Joumal of Jouma, 3/(3), 351-357.

Zhang, M., Katzman, R., Salmori, D., Jin, H., Can, G. Wang, Z, Qu, G, Grant, I., Yu, E., Levy, P, Klauber, M. R., L Lit, T. (1990). The prevalene of dementia and Alzheimer"s discase in Shanghai, China: Impact of age, gender, and education. Antals of Newrotogy, $27(4), 428-437$. 


\title{
Chapter 5
}

\section{Effect of Test Duration on Age-Related Differences in Stroop Interference*}

\begin{abstract}
The effect of test duration on age-related differences in Stroop interference was assessed in a large cross-sectional study involving 429 healthy subjects in four age groups (young. 25-35 years; young middle-aged, 40-50 years; old middle-aged, 55-65 years; old, 70-80 years). The results show a clear effect of test duration on Stroop interference. The Stroop Color Word Test was run in two parts. The young group performed the first part relatively rapidly but became slower in the second test part, whereas the old group showed the reverse effect. The two middle-aged groups did not show differences between the first and second test parts. The results are interpreted in terms of a deficit in response inhibition by a controlled processing strategy. A psychological interpretation of the study findings in terms of increased cautiousness appears less probable. The first part of the test, an abridged version of the test, may prove superior to the regular version for both clinical and research purposes.
\end{abstract}

* Part of this chapter has been published as:

Klein, M., Ponds, R. W. H. M., Houx, P. J., Jolles, J. (1997). Effect of Test Duration on Age-Related Differences in Stroop Interference. Jownal af Clinical and Experinental Neuropsychology, 18(1), 77-82. 


\subsection{Introduction}

One of the most widely used tests of visual selective attention is the Stroop Color Word Test (SCWT; Stroop, 1935). Since the intial introduction of the SCWT more than 700 studies have sought to explain nuances in the Stroop effect (MacLeod, 1992). MacLeod suggests two reasons for the popularity of the Stroop test: its large and always statistically reliable effect and the abundance of possible explanations for the effect.

Although the Stroop test exists in various forms, the essence of the test is that performance in several subtests is compared. The color-word interference subtask requires the subject to name a set of colors when they are printed as letters in a conflicting color (e.g., the word "blue" printed in green ink). Performance on this subtask is compared with performance on other subtasks in which the subjects read color words printed in black or in the appropriate colored ink. Stroop (1935) found that subjects take Ionger to perform the color-word interference subtask than the other two subtasks. Generally, the difference in time needed is thought to be because the automatic reading response interferes with the response required by the task and can be taken as a measure of selective attention. In an extensive review, MacLeod (1991) summarized and evaluated the existing theoretical positions and concludes that earlier theories that attempted to identify a single bottleneck in attention were less successful in explaining the Stroop effect than more recent theories based on parallel processing of relevant and irrelevant information (Cohen, Dunbar, \& McClelland, 1990; Cohen, Servan, \& McClelland, 1992).

The SCWT appears to be very sensitive to age-related slowing and interference effects. In a lifespan study with subjects aged from 7 to 80 years, Comalli, Wapner, and Wemer (1962) concluded that interference begins early in the school years, rising to its highest level as reading skills develop. With continuing development of reading skills, interference declines until approximately age 60 , at which point it begins to increase again. This increase in interference after age 60 has been confirmed by others (Cohn, Dustman, \& Bradford, 1984; Panek, Rush, \& Slade, 1984). In addition to age, health-related factors appear to have a strong effect on Stroop interference in elderly subjects. Houx and Jolles, 1994, and Houx, Vreeling, and Jolles (1993) have recently shown that Stroop Interference is increased by more than $100 \%$ in subjects older than 60 who are bealthy according to accepted gerontological criteria, but who are characterized by a so-called "Biological Life Event" (BLE). BLE are factors with a potential, but up till now umproven, influence on the brain. Mild traumatic brain injury and operation under general anesthesia are examples of BLE (Houx, \& Joiles, 1994; Houx et al., 1993; Jolles, Houx, Vreeling, \& Verhey, 1993).

Apart from age, suboptimal health, practice (Dulaney \& Rogers, 1994), and personality factors also may influence performance on the Stroop test, especially interference. Several authors (e.g., Botwinick, 1977) suggest that people, as they grow older, become more cautious and therefore slower but more accurate. Cautionsness appears to play a role in visual selective attention tasks, as was suggested by Rush, Panek, and Russell (1987). This notion, however, was not supported by the results of the study by Houx et al. (1993), who found that older subjects made more errors, and especially old individuals with BLE.

Because of the many factors that appear to influence performance on the SCWT, there are various explanations for the Stroop interference effect seen with age. Most interpretations suggest that the disproportional interference is associated with age-related changes in the 
efficiency of stimulus encoding and/or response selection levels of processing (for reviews, see Duncan-Johnson, 1981; Rogers \& Fisk, 1991). In a P300 study, Duncan-Johnson and Kopell (1981) suggested that the decrease in response speed might be caused by response competition that is triggered by the automatic activation of lexical processing, whereby slowing of the response output is greater among elderly people than among the young people.

Since reading abilities and lexical processing tend to be correlated with intelligence, this source of individual variation should also be considered as a factor probably infuencing speed on the SCWT. Studies that investigated the relation between intelligence and SCWT speed, however, mainly focused on children (Pati \& Dash, 1990a; Pati \& Dash, 19906). In their study of adults aged 55 - 81 years, Rush, Panek, and Russell (1990) identified, among others, verbal intelligence as a factor contributing to a systematic variation in SCWT response patterns.

The present study was devised to study the effect of test duration. If subjects have to perform an interference task for a prollonged period of time; the ability to inhibit distractor information might be affected in older subjects due to a decline in the availlability of processing resources (Salthouse, 1988a). Because of age-related differences in the ability to allocate resources and the ability to inhibit distractor information, we hypothesized that test duration would differently affect the performance of young and old subjects. On the basis of the results of a first experiment, we compared the mean performance of 429 healthy subjects in four age groups (young, young middle-aged, old middle-aged, and old) on the first $40 \%$ of the test with that on the remainder of the test.

\section{2}

\section{Method}

\section{2 .1}

Subjects and Procedure

This study was conducted as part of a large cross-sectional study into biological and psychological determinants of cognitive aging, the Maastricht Aging Study. The procedure for recruitment of the subjects has been described in detail elsewhere (Jolles, Houx, Van Boxtel, \& Ponds, 1995). In short, 679 normal, healthy subjects were drawn from a pool of 1.252 individuals who volunteered for the test program in a postal survey from which medical and demographic information was also derived. Subjects were randomly recruited from a register of general practices (Metsemakers, Höppener, Knottnerus, Kocken, \& Limonard, 1992). Medical exclusion criteria were defined as those active or inactive medical conditions that may interfere with normal cognitive function: cerebrovascular disease, all CNS tumors and congenital malformations, multiple sclerosis, parkinsonism, epilepsy, dementia or other organic psychosis, schizophrenia, affectiwe psychosis, and mental retardation. In addition, before participation in the test program all participants were screened by means of a semistructured interview to update and check for the following exclusion criteria: history of transient ischemic attacks, brain surgery, hemodialysis for renal failure, electroconvulsive therapy, and chronic use of psychotropic drugs. Subjects with active cardiovascular or pulmonary disease were excluded, which means that subjects with previous, but not active, conditions were included. Subjects were stratified by age ( 12 discontinuous age classes: 25 years \pm 1 to 80 years \pm 1 ) and sex. Subjects were subsequently assigned to one of four age groups (25-35, young; 40-50, young middle-aged; 55-65, old middle-aged, and 70-80 years, old). Eighty-five of 679 subjects were excluded due to frailty or chronic psychotropic medicine use; 104 subjects 
whitrew because of the expected personal investment of time and effort. The exciusion percentages for the four age groups were $2.3,3.3,4.4$, and 8.9 , respectively. Stroop data were thus obtained for 470 subjects. The Stroop data of 4 subjects were invalid or unreliable. Furthermore, 17 subjects were excluded as statistical outhers. Outlers were defined as responses of more than $3 \mathrm{SD}$ below the mean for lines 14 or for lines $5-10$ of either Stroop subtask. Intelligence was measured with four subtasks of the Groningen Intelligence Test (GTT; Luteijn \& Van der Ploeg, 1983). This abridged version showed a correlation of 94 with the complete test (Lutejn, 1966). Intelligence was not equally distributed across all age groups ( $F$ $(3,449)=4.12, p<.01)$, subjects in the oldest group were significantly more intelligent than subjects in the other age groups. Since IQ might influence response time, the four age groups were matched for intelligence. This was done by exeluding 21 of the initial 92 subjects from the oldest age group; these subjects had an 10 score above 127 . The analyses were based upon the data of the remaining 429 subjects. Subject characteristics are summarized in Table 5.1.

Table 5.11

Demographic Characteristics of Normal, Healthy Subjects $(N=429)$

\begin{tabular}{lccccccc}
\hline & & $N$ & \multicolumn{2}{c}{ Age } & \multicolumn{2}{c}{ Intelligence } & Sex (M/F) \\
Suljijects & $n$ & $(\%)$ & $M$ & $S D$ & $M$ & $S D$ & $\%$ \\
\hline Total group & 429 & $(100.0)$ & 50.3 & 16.1 & 113.0 & 12.2 & $49 / 51$ \\
Young & 121 & $(28.2)$ & 30.6 & 4.2 & 112.3 & 10.4 & $50 / 50$ \\
Young middle-aged & 119 & $(27.7)$ & 45.6 & 4.2 & 112.5 & 12.6 & $49 / 5 !$ \\
Old middle-aged & 118 & $(27.5)$ & 60.7 & 4.2 & 113.0 & 12.3 & $49 / 51$ \\
Old & 71 & $(16.6)$ & 74.3 & 3.9 & 113.0 & 10.1 & $45 / 55$ \\
\hline
\end{tabular}

\subsubsection{Measures}

\section{Srroop Color Word Test (SCWT)}

The SCWT was very similar to the test used by Nehemkis and Lewinsohn (1972) and Panek et al. (1984). "Three cards of $10 \times 10$ items were used. The first Card (I) consisted of 100 color words (green, red, yellow and blue) printed in black ink and randomly arranged in 10 lines of 10 items. The subjects were asked to read the words as fast as possible. The second Card (I) consisted of colored patches (green, red, yellow, and blue) of $0.7 \times 2.0$ centimeters, again in a random order. Subjects were requested to name the colors as quickly as possible. Card III involved color names again, but the printing ink was different from the color name (e.g., the word yellow is printed in green ink). The subjects were asked to name the color of the printing ink and not to read the word.

\section{Abridged Stroop Test (SCWT-A)}

A first experiment was performed to assess the correlation between the performance on the regular Stroop test ( 10 lines) with that on shorter versions (1 through 9 lines). This experiment was done to determine whether, and up till where, the regular Stroop test could be abridged. Thirty male and 40 female healthy volunteers (age range between 40 and 89 years; mean age 65.2 years, $S D=11.3$ ) performed the Stroop test. The time needed for each line of SCWT 
cards I, II and III was measured. Subsequently, the time for each line was correlated whth the total time needed to finish Card I, II or III. Results are shown below.

In order to detect differences in processing speed as a function of age, we calculated the mean time needed to complete a line in the first part of the subtest ( 4 lines divided by 4 ) and for the second part (the remaning 6 lines divided by 6 , derived by subtracting the time needed for 4 lines from the total time of either subtest). The condition in which we used the first 4 lines of Card I, II or III will be referred to as SCWT-A (for abridged) I, I or III.

On the basis of these results, the Stroop test was used in the main study and performance time for both the SCWT-A I, II, III, and SCWT Cards I, II, III was measured. Furthermore, we measured the total number of errors and spontaneous corrections on SCWT Cards II, II, and III. SCWT Cards I, II, and III are referred to as SCWT I, II, and III.

The variables used for the analysis were: the mean time per line needed for completion of SCWT-A, for the remainder of the test (the final 6 lines) and SCWT I, II, or II and the interference score: $(\mathrm{tIII}-.5 *(\mathrm{tI}+\mathrm{tII})) /(.5 *(\mathrm{tl}+\mathrm{tII})) * 100 \%$ (t denoting the time needed for the completion of either subtest). The same procedure was carried out for the second test part ( 6 lines). This measure relates the extra time needed for subtest III to the average time needed for the first two cards. Since subjects older than approximately 60 years tend to be slower on SCWT III, the use of the ustal interference measure (IIII - III) would be an overestimation of the actual interference in older people (Houx et al, 1993). A speed ratio was calculated by dividing the mean time needed for the first test part by the time needed for the second part. A score of 1 thus means that subjects perform equally well on the first and second parts of a card.

As far as age (four groups) is concerned, one-way ANOVAs were performed on the SCWT" main variables. The level of significance was set al $p<.05$, to deternine test variables liable for subsequent post-hoc analysis. Duncan's post-hoc multiple group comparisons were made to detect differences between age groups $(p<.05)$. Sex differences in relation to the main test variables were analyzed by using unpaired T-tests (two-tailed, $p<.05$ ). In order to decide which intra-individual factors contributed to SCW'T performance when composing normative data, we entered age, IQ, and sex in a stepwise hierarchical multiple regression model. The dependent variables were SCWT-A I, II, and III.

\section{3}

\section{Results}

\section{Experiment 1: Correlations between SCWT-A and SCWT}

The correlations between performance on the SCWT with that on shiorter versions (1 through 9 lines) of the test are depicted in Figure 5.1. The correlation between performance on the SCWT and an abridged version with 3 or more lines appeared to be remarkably high (Pearson's $r$ : Card I, $r=.93, p<.001$, Card II, $r=.92, p<.001$, Card II, $r=.94, p<.001$, respectively).

Performance on the 3 -line abridged version accounted for $81.0 \%, 86.5 \%$, and $60.8 \%$ of the total explained variance for the subtests I, II and III, respectively. These figures were $86.5 \%$, $90.3 \%$, and $74.0 \%$ for the 4 -line version. From 6 lines onward, the three subtests each accounted for more than $92.2 \%$ of the explained variance. On the basis of these data we decided to use the 4-line version of the SCWT in the main study. The non-maximal correlation 
between the regular SCWT and the 4-line version (SCWT-A) would enable us to analyze systematic differences between the performance of the four age groups on the first part of the Stroop ( 4 lines) wersus the second part ( 6 lines).

Iugure 5.1 (left): Correlation berween thr the needed to complete subseguent lines on Stroop Color Word Test Cards I. II and III with the total dime meded to complete the entire card.

Figure 5.2 (right): Mean perfomance of subjects (aged 25-80 years) for the furst tour lines (SCWT $1-4$ ) and the remaning six Ines (SCWT 5 -10) of the Stroop Color Wond Test. Vertical bars denote Standard Errors of the Mean.

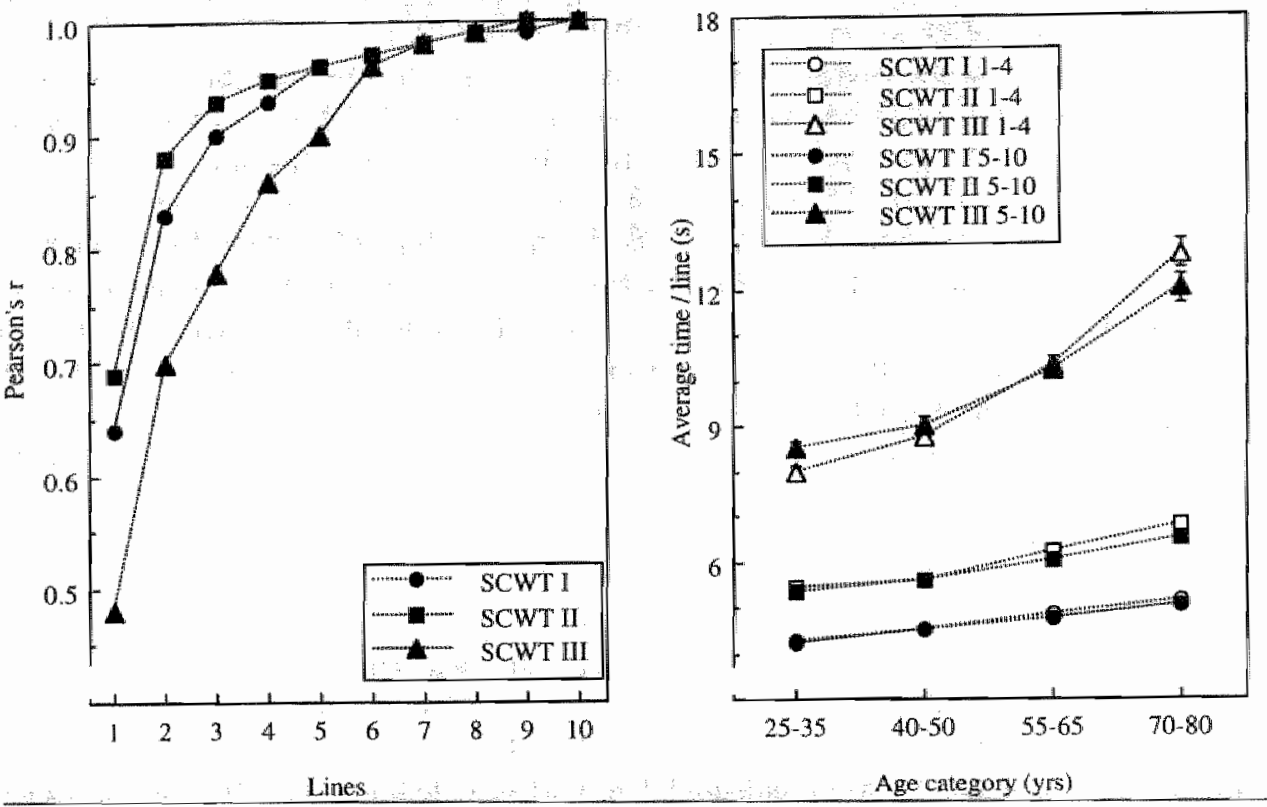

Experiment 2: SCWT A and SCWT in 429 healthy subjects

One-way ANOVAs carried out on the main variables yielded essentially similar age effects for SCWT-A I, II, and III $(F(3,428)=22.32, p<.001,(F(3,425)=36.10, p<.001$, and $F(3$, $427)=88.59, p<.001$, respectively $)$ and SCWT I, II, and III $(F(3,428)=25.95, p<.001)$, $F(3,428)=35.15, p<.001$, and $(F(3,426)=71.89, p<.001)$. With respect to the total number of errors and spontaneous corrections, no differences were found in SCWT I and II among the different age groups (errors I, II: both $F(3,427)<1$; spontaneous corrections I: $F$ $(3,427)<1$. II: $F(3,427)=1.11$, n.s.). This did not seem to be the case for SCWT III, where subjects between 70 and 80 years performed less well than the younger subjects (errors: $F(3,427)=5.36, p<.01$; spontaneous corrections: $F(3,427)=5.61, p<.01)$.

Male and female participants took the same time to complete SCWT I, II, and III: $t=-1.42, d f$ $=427$, n.s., $t=.93, d f=426, \mathrm{n} . \mathrm{s}$. and $t=10$, $d f=425$, n.s., respectively). Interference scores of SCWT were also not affected by sex $(t=.20, d f=425$, n.s. $)$. 
Figure 5.3 (left): Mean speed ratios of subjects aged 25 to 80 years on the Stroop Color Word Test. Vertical bars denote Standard Errors of the Mean. Speed ratio is calculated by dividing the mean time needed to complete the first test part by the mean time needed to complete the second part: a score of I thus means that subjects perform equally well on the first and second parts of a card.

Figure 5.4 (right): Mean interference scores of subjects of age 25 to 80 years on the tirst test part (4 lines) and the second test part (the remaining 6 lines) of the Stroop Color Word Test. Vertical bars denote Standard Errors of the Mean.
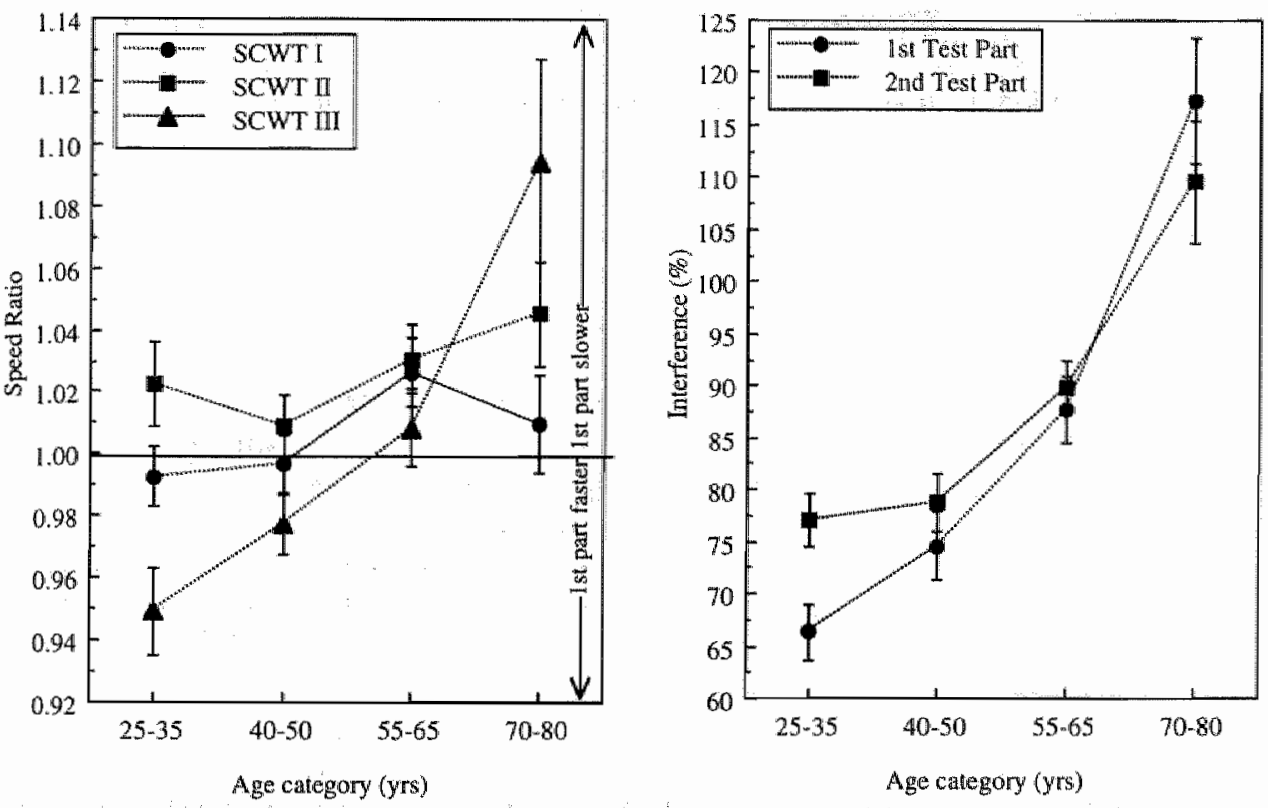

The main question of the present study was the possible effect of age on performance on the first 4 versus the last 6 lines of the SCWT. The mean time per line for the four age groups is depicted in Figure 5.2. Two-way ANOVAs (Age $x$ Time) on these data showed significant age effects for the three subtests $(F(3,425)=25.71, p<.001 ; F(3,422)=37.15, p<.001 ; F(3$, $422)=76.27, p<.001$, respectively). A significant time effect was found for the second subtest $(F(1,422)=12.35, p<.001)$ and a significant Age $\mathrm{x}$ Time interaction for SCWT III $(F(3,422)=12.71, p<.001)$, and a mear significant Age $x$ Time interaction for SCWT II ( $F$ $(3,422)=2.55, p=.055)$.

The speed ratio for the first versus the second test part for the four age groups is given in Figure 5.3. The youngest group needed relatively more time to perform the second part of the test, whereas the opposite was true for the oldest group. The oldest group typically became faster on SCWT III from the first to the second test part. Accordingly, the Interference score for the young group increased from the first to the second test part (paired $t$-test; $t=-3.91, d f=119, P$ $<.001$ ) whereas it decreased in the oldest group $(t=1.37, d f=69, \mathrm{n.s}$. $)$. The middle-aged subgroups showed no differences in interference from the first to the second test part (Figure $5.4 ; t=-1.91, d f=115$ n.s.; and $t=-.89, d f=117$; n.s.). 


\section{Normative data}

The results of the stepwise hierarchicat multiple regression analysis on SCWT-A I, II, and III $(\mathrm{PDN}=.05$ ) are summarized in Table 5.2. For color word reading (SCWT-A I) age and intelligence accounted for $21.0 \%$ of the total explained vanance, suggesting that subjects tend to need more time with increasing age. Morcover, the more intelligent the subject, the shorter the time needed to complete this task. Sex, according to criterion, was not included in the model. Color naming (SCWT-A II) was affected by age to a greater extent, as this variable could explain $20.0 \%$ of the total wariance, at the second step, IQ added another $3.4 \%$. Sex, on the thitd step, added another $1.5 \%$.

Table 5.2

Strepwise Hierarchical Multiple Regression for the Stroop Color Word Test

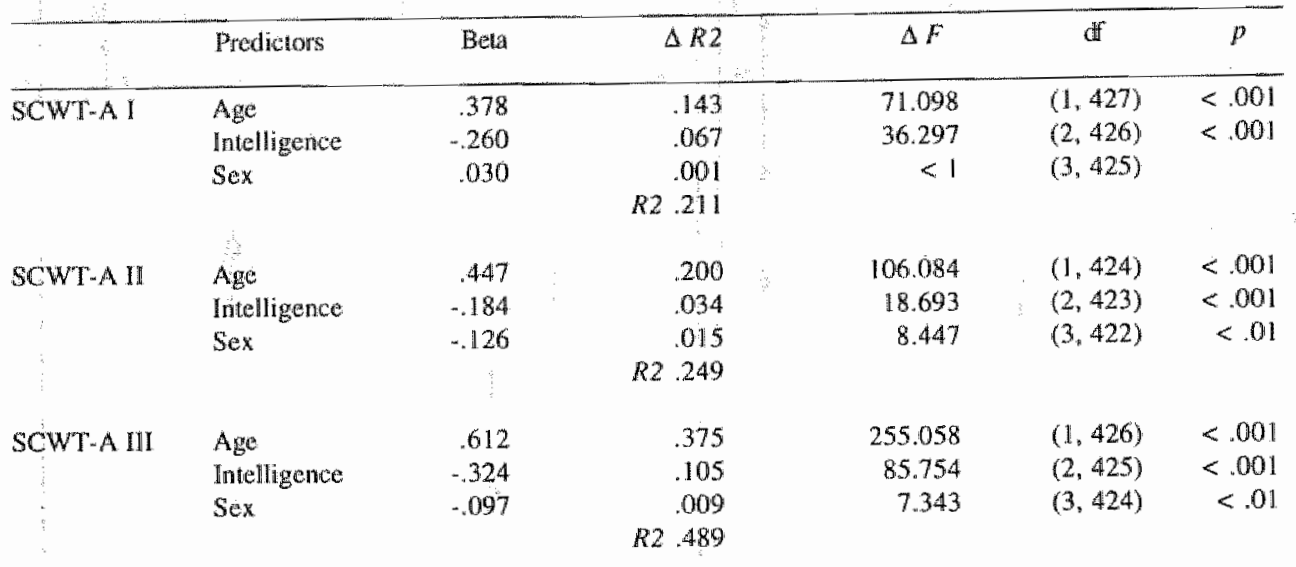

Note. $\Delta R 2$ denotes the charge in $R 2$ with the introduction of either predictor; $\Delta F$ denotes the $F$-value for the predictor that is entered in the regression model; $R 2$ denotes the total proportion of variance that is explained by all predictors entered in the model. Male subjects are denoted by a " 1 "; female subjects are denoted by a " 2 ".

In color-word interference (SCWT-A III), age could explain an even higher percentage of the total variance than in the two previous SCWT-A conditions, namely $37.5 \%$; at the second step, $1 Q$ added another $10.5 \%$. Sex, on the third step, added another $9 \%$. Regression analysis showed that sex had a negligible influence on SCWT performance when compared to the influence of age. Intelligence accounted for $20-25 \%$ of the variance, when compared to age. Normative data for SCWT-A and SCWT were formalated for the four age groups (see appendix).

\subsection{Discussion}

The aim of the present study was to assess the effect of test duration on age-related differences in Stroop interference. The results show the well-known effects of age on the speed of color word reading, color naming, and color-word interference. There was a clearcut effect of test duration: the young group was relatively fast on the first test part of the interference test but slower on the second test part. In contrast, the old group was relatively slower on the first test 
but speeded up on the second test part. The slope for the furst lest part (Figure 5.2) was 0.51 and for the second test part 0.41 . Thus the first test part could distinguish beween the age groups better than the second test part: the time needed by old subjects was 1.64 times that of young subjects for the first 4 lines, but 1.23 times for the second 6 lines.

Our results have several implications. In the first place, the abridged version of the Stroop test may prove useful in the clinical setting. Elderly subjects and especially neurological patients frequently complain that they find it very difficult to complete the task. Especially patients with increased susceptibility to interference, e.g. patients in early stages of dementia, have to invest a considerable amount of effort when doing the Stroop test. This makes testing a difficult and stressful experience. A Stroop test consisting of 10 lines of 10 words each can be regarded as a cognitive task which requires the subject to apply a controlled processing strategy for a series of 100 consecutive trials. The negative impact of sustained effort may be reduced by the use of the SCWT-A, since this version takes $60 \%$ less time to administer. Many currently used neuropsychological tests are long, and in fact the SCWT is among the shorter attentional tests in regular use (Lezak, 1995). Thus it is very probable that the influence of test duration shown in the present study will also be present in other tests. If so, it would be important to shorten existing tests, in order to improve the differentiating power and to reduce the total testing time. There are various explanations for our findings. One possible explanation of the "slow starting" phenomenon in older subjects could be that older subjects tend to be more cautious. "This is especially true for new or unfamiliar situations, as is the case in subtest III. The studies by Rush et al. $(1987,1990)$ indicate that cautiousness in older subjects is manifested more in terms of accuracy than in terms of response speed, and that the level of cautiousness increases with increasing age. They suggest that more cautious older adults can be expected to exhibit significantly slower response times, particularly on subtest III, and to commit significantly fewer errors on the Stroop test. In our study, however, besides longer response times, we also found that the old subjects made more spontaneous corrections and errors on SCWT III than the other subjects did. Thus although older subjects probably process distractors in a more cautious way, they still fail to employ inhibitory mechanisms to suppress the distracting stimuli. This assumption is consistent with the results of Kane, Hasher, Stolzfus, Zacks, and Connelly (1994), who compensated for the age-related slowing of information processing and still found that older subjects could not benefit from inhibitory mechanisms when confronted with distracting stimuli.

Apart from increased cautiousness, it is also possible that the slow-starting phenomenon is related to the fact that elderly subjects are less good at developing new automatic processes or at adapting existing automatic processes, as is suggested by Dulaney and Rogers (1994). Younger subjects become slower because they can only sustain active and efficient information processing for a limited time. Described in terms of processing strategies (Shiffrin \& Schneider, 1977), young subjects may be able to complete the first lines very rapidly because of a controlled processing strategy requiring extra mental effort and the availability of more cognitive processing resources (Salthouse, 1988a, 1988b). When the task duration increases, these resources may become exhausted, which gives rise to a decreased speed of performance. The more recent theories on parallel processing of relevant and irrelevant information (Cohen et all, 1990,1992 ), which stress the notion that these processes might change with aging, appear to be especially promising.

In conclusion, the present finding of a clear effect of test duration on the effect of age on Stroop interference may be relevant in terms of both possible clinical applications and improved 
understanding of the neurocognitive processes underlying Stroop interference. These results suggest new aventies for the investigation of this intriguing phenomenon and of neurocognitive changes with age.

\section{5}

\section{References}

Botwinck, J. (1977). Intellectual abilities. In H. Brren \& Kchaie (Eds.), Handbook of the Psychology of Aging (Pp. 580-605) New York: Van Nostrand Reinhold.

Cohen, J, D. Dumbar, K., McCleliand, I. L. (1990). On the control of automatic processes: A parallel distributed processing account of the Strop effect. Psychological Review, 97(3), 332-361.

Cohon, J. D. Servan, S. D., McClelland, I. L. (1992). A parallel distributed processing approach to automaticity. Special Issue Views and varieties of automaticity. American Journal of Psychology, 105(2), 239.269

Cohn, N, B., Dustman, R. E., Bradford, D. C. (1984). Age-related decrements in Stroop Color Test performance. Joumal of Clinical Pychology, $40(5), 1244 \cdots 1250$.

Comalli, P. E. J., Wapner, S., \& Werner, $\mathbb{H}$ (1962). Interference effects of Stroop color-word test in childhood, adulhood, and aging: Journal of Cenetic Psychology, 100, 47-53.

Dulaney, C. L., \& Rogers, W. A. (1994). Mechanisms umderlying reduction in Stroop interference with practice for young and old adults. Joumal of Experimental Psychology: Learning, Memory and Cognition, 20(2), 470-4 84.

Duncan-Johnson, C. C. (1981). P300 latency: A new metric of information processing. Psychophysiology. 18(4523), 207-215.

Duncan-Johnson, C. C., \& Kopell, C. S. (1981). The Stroop effect: Brain potentials localize the source of interfierence, Science, $214,938-940$.

Houx, P. J., \& Jolles, J. (1994), Vulnerability factors for age-related cognitive decline. In $R$. L. Isaacson \& $\mathrm{K}$. F. Jensen (Eds), The Vulterable Brain and Environmental Risks, (Voll. vol. 3, pp. 25-4l). New York: Plenum Pressi.

Houx, P. J., Vreeling, F. W., \& Jolles, J (1993). Stroop interference: Aging effects assessed with the Stronp Color Word test. Experinental Aging Research, 19(3), $209-224$.

Jolles, J., Houx, P. J., Van Boxtel, M. P. J., \& Ponds, R. W. H. M. (Edss.). (1995). Maastricht Aging Study. Determinants of Cognitive Aging. Maastricht; The Netherlands: Neuropsych Publishers.

Jolles, J., Houx, P. J., Vreeling, F. W., \& Werhey, F. R. J. (1993). Cognitive aging, biological life events and primitive reflexes. Neuroscience Research Comunuications, 13(Suppl. 1) 47-50.

Kane, M. J., Hasher, L. H., Stolzfus, E. R. Zacks, R. T., \& Connelly, S. L. (1994). Imhibitory attentional mechanisus in aging. Pychology and Aging, 9 (1), $103-112$.

Lezak, M. D. (1995). Nenropsychological assessment ( 3 ed., New York: Oxford Uniwersily Press.

Luteijn, F. (1966), Een nieuwe verkorte GIT" (A new abridged Groningen Intelligence Test). Nederlands Tijdschrift voror de Psychologie on haar Crensgebreden, 2/(10), 675-682.

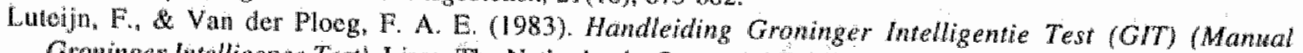
Grominger litelligence Test). Lisse, The Netherlands: Swets and Zeitlinger.

Macleod C. M. (1991). Half a century of research on the Stroop effect: An integrative review, Psychological Bulletin, $109(2), 163-203$

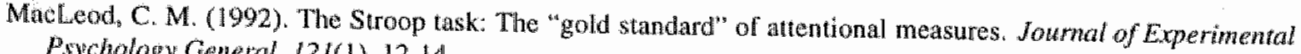
Psychology General, $12 /(1), 12-14$

Metsemakers, J. F. M., Hoppener, P., Knotmerus, J. A., Kocken, R, J. J., \& Limonard, C. B. G. (1992) Computerized health information in the Netherlands: A registration network of family practices. British Journal of General Practice, 42(356), 1.02-106.

Nehemkis, A. M. \& Lewinsohn, P. M. (1972). Effect of left and right cerebral lesions in the naming process. Perceptwal and Motor Skilis, 35(3),787-798

Panck, P. E. Rush, M. C... \& Siade, L. A. (1984). Locus of the age-Stroop interference relationship. Joumal of Genetic Psychology, $145,209-216$

Pati, P., \& Dash. A. S. (1990a). Effects of grade, sex and achievement lewells on intelligence, incidental memory and Stroop scores. Psychological Studies, $35\left(1 j_{1} 36-40\right.$.

Pati, $P_{.,}$Dasln A. S. (1990b).. Intertelationships between incidental memory, non-verbal intelligence and Stroop soores. Psycho Lingutio, $20(1), 27-31$. 
Rogers, W. A., \& Fisk, A. D. (1991). Age-related differences in the maintenance and nindification of automatic processes: Arithmetic Stroop interference. Human Factors, 3.8(1), 45-56.

Rush, M. C., Panek, P. E., \& Russell, J. E. (1987). Cautiousness and visual selective attention performance among older adults. Journal of Genetic Psychology, 148(2), 225-235.

Rush, M. C., Panek, P. E., \& Russell, J. E. (1990). Analysis of individual variability among older adults on the Stroop Color Word Interference Test. International Joumal of Aging and Haman Developmeth, 30(3), 225236.

Salthouse, T. A. (1988a). Initiating the formalization of theories of cognitive aging. Psychology and Aging. $3(1), 3-16$.

Salthouse, T. A. (1988b). Resource-reduction in interpretations of cognitive aging. Developmental Review, 8 , 238-272.

Shiffrin, R. M, \& Schneider, W. (1977). Controlled and automatic human information processing: II. Perceptual learning, automatic attention and a general theory. PSychological Review。 84, 127-190.

Stroop. J. R. (1935). Studies of interference in serial verbal reactions. Joumal of Experimental Psychology" 18, $643-662$. 


\section{Appendix}

Normative data of SCWT.A I, II, and III and SCWT I, II, and III with respect to age

\begin{tabular}{|c|c|c|c|c|c|c|c|c|c|c|}
\hline \multirow[t]{2}{*}{ Age } & \multicolumn{2}{|c|}{$S C W T$} & \multicolumn{8}{|c|}{ Percentile Score } \\
\hline & 8 & $90 \mathrm{th}$ & 80 th & $70 \mathrm{th}$ & $60 \mathrm{hh}$ & 50 th & $40 \mathrm{th}$ & 30 th & $20 \mathrm{th}$ & $10 \mathrm{hr}$ \\
\hline \multirow{6}{*}{$22-37$ Years } & A I & 13.6 & 14.6 & 15.3 & 15.7 & 16.3 & 17.1 & 18.1 & 19.2 & 20.8 \\
\hline & A II & 17.2 & 18.9 & 19.5 & 20.1 & 21.1 & 22.1 & 23.3 & 24.7 & 26.5 \\
\hline & A III & 24.6 & 26.8 & 28.1 & 30.0 & 33.0 & 33.1 & 34.2 & 36.7 & 40.7 \\
\hline & I & 34.6 & 36.9 & 38.7 & 40.2 & 41.8 & 43.5 & 45.5 & 46.8 & 52.0 \\
\hline & II & 43.4 & 46.1 & 49.5 & 51.5 & 53.3 & 55.2 & 58.2 & 60.7 & 65.3 \\
\hline & InI & 65.8 & 69.7 & 73.1 & 77.5 & 80.7 & 84.8 & 90.3 & 97.1 & 105.0 \\
\hline \multirow[t]{6}{*}{38.52 Years } & AI & 14.7 & 15.1 & 16.0 & 17.0 & 18.0 & 18.5 & 19.3 & 20.3 & 22.3 \\
\hline & A II & 18.5 & 19.2 & 20.4 & 21.1 & 22.0 & 22.7 & 23.6 & 24.9 & 26.2 \\
\hline & A III & 25.4 & 27.4 & 30.9 & 32.4 & 338 & 35.2 & 37.0 & 40.3 & 48.9 \\
\hline & I & 37.9 & 40.0 & 41.2 & 43.0 & 44.4 & 45.7 & 47.0 & 50.0 & 54.1 \\
\hline & II & 46.4 & 48.4 & 51.1 & 52.9 & 54.8 & 57.2 & 59.7 & 62.3 & 68.0 \\
\hline & $\mathrm{mII}$ & 67.3 & 72.3 & 78.7 & 83.6 & 85.8 & 88.3 & 95.7 & 101.9 & 115.4 \\
\hline \multirow[t]{6}{*}{$53-67$ Years } & A I & 15.7 & 16.5 & 17.3 & 18.1 & 18.9 & 19.5 & 20.7 & 21.9 & 23.8 \\
\hline & A II & 19.4 & 21.2 & 22.7 & 23.4 & 24.5 & 25.5 & 26.6 & 28.0 & 30.5 \\
\hline & A III & 32.5 & 34.3 & 36.1 & 37.7 & 38.9 & 41.1 & 43.8 & 48.5 & 54.3 \\
\hline & I & 39.2 & 41.3 & 43.8 & 45,3 & 47.0 & 48.8 & 50.8 & 53.4 & 58.2 \\
\hline & III & 48.6 & 53.6 & 56.8 & 58.9 & 50.6 & 62.1 & 64.1 & 67.1 & 73.2 \\
\hline & IIII & 82.3 & 87.0 & 90.0 & 94.0 & 99.1 & 103.0 & 109.9 & 119.3 & 13.1 .1 \\
\hline \multirow[t]{6}{*}{$68-83$ Years } & A I & 16.6 & 17.5 & 18.3 & 18.9 & 19.6 & 20.3 & 22.0 & 24.1 & 26.2 \\
\hline & A II & 21.4 & 23.3 & 24.9 & 25.8 & 27.9 & 28.4 & 29.4 & 30.4 & 32.7 \\
\hline & A III & 37.6 & 40.9 & 45.2 & 47.5 & 50.4 & 52.1 & 56.1 & 60.4 & 65.3 \\
\hline & I & 43.7 & 44.7 & 45.9 & 47.1 & 49.2 & 51.0 & 53.6 & 57.9 & 62.7 \\
\hline & II & 52.2 & 58.3 & 60.5 & 64.6 & 66.9 & 68.1 & 70.4 & 73.3 & 78.0 \\
\hline & III & 89.5 & 100,6 & 111.4 & 117.7 & 120.5 & 125.1 & 133.7 & 147.8 & 160.3 \\
\hline
\end{tabular}




\title{
Chapter 6
}

\section{The Self-Paced Serial Addition Task (SPASAT) and Aging:}

\author{
Predictors of Performance in Divided Attention*
}

\begin{abstract}
This study aimed at detecting the deteminants of the age-associated decrease in divided attention on a self-paced serial addition task, the SPASAT We furthermore examined whether SPASAT performance was affected by time-on-task effects. The SPASAT was administered to 196 subjects in four age groups (young, 25-35 years; young middle-aged, 40-50 years; old middle-aged, 55-65 years; old, 70-80 years). Overall age effects were found on response time (RT), number of errors and restarts. Simple RT, arithmetic ability, primary memory capacity, concept shifting ability, interference susceptibility, and degree of hearing loss contributed to performance. Speed of performance as a function of time-on-task improved in the two younger groups, but not in the older groups. Repeated test administration was not associated with a reduction in the number of errors in the oldest group, but the number of restarts declined in the youngest and the oldest groups, but for different reasons. No evidence was found for age-related differences in speed-accuracy trade-off mechanisms. Elderly subjects appear to be more able to monitor their own cognitive performance, leading to a more stable performance over time. The ease of administration makes the SPASAT a very suitable instrument for measuring attentional functions in large-scale studies of cognitive aging.
\end{abstract}

* Klein, M., Ponds, R. W. H. M., Jolles, J. Submitted for publication. 
Perhaps the most ubiquitous finding in the study of cognitive aging is that response time (RT) slows with advanced age (Salthouse, 1985). Divided attention efficiency also appears to decline with age, but this conclusion is affected by the task and methodology that are used. Several mechanisms have been proposed to explain why older subjects may be more vulnerable to divided attention or dual-task conditions. Crossley and Hiscock (1992) suggested that at reduction in general-purpose processing resources might be affected in the process of aging (see also McDowd \& Craik, 1988; Plude \& Hoyer, 1986). Salthouse, Rogan, and Prill (1984) suggested that either the total amount of attention available or the efficiency of its allocation decreases with age even when corrected for single-task performance. In 1995, however, Salthouse, Fristoe, Lineweaver, and Coon (1995) found the effects of task complexity to be relatively small, and considered the mechanisms responsible for age-related effects on measures of both simple and complex performance to be related to the slower speed at which elementary operations are executed. Madden, Connelly, and Pierce (1994) suggested that the presence of distracting information compromises the performance of older subjects, a suggestion that is in line with the model of Hasher, Stoltzfus, Zacks, and Rypma (1991) and Hasher and Zacks (1988), who considered inhibitory control as a central mechanism determining the content of working memory and consequently influencing cognitive functions.

The generalized decline in fundamental neural events, the so-called "general slowing" (Birren, Woods, \& Williams, 1980; Cerella, Poon, \& Fozard, 1981) does not appear to be the only factor affecting divided attention performance with increasing age. With regard to memory, Lorsbach and Simpson (1988) suggested that retrieval and comparison of category information is particularly demanding for older adults, while Greenwood and Parasuraman (1991) showed that a decreased divided attention performance in older subjects might mainly be caused by greater attentional costs for the late stages of information processing, which involve matching and response selection. Park, Smith, Dudley, and Lafronza (1989) furthermore suggested that free recall and clustering performance in older subjects decline more than those of young subjects when attention is divided at encoding, but not at retrieval. When information requires consciously controlled processing, as opposed to automatic processing (Shiffrin \& Schneider, 1977), age and divided attention appear to have a detrimental effect on memory processing (Jennings \& Jacoby. 1993). When divided attention tasks are presented aurally, a significant proportion of the variance explaining the age-related decline in cognitive performance can be accounted for by hearing defects (Rabbitt, 1991; Van Rooij \& Plomp, 1991). This suggests that perceptual loss at peripheral levels somehow appears to affect processing at central levels. This is a rather intriguing phenomenon, all the more because these studies have controlled for initial differences in perceptual acuity, which also declines with aging. Immediate memory performance in old subjects appears to be differentially decreased when speech rates are very fast, as was suggested by Tun, Wingfield, Stine, and Mecsas (1992), who furthermore noted that an age-related reduction in working memory efficiency contributes to age differences in divided attention processing (Tun, Wingfield, \& Stine, 1991).

Another major factor that might specifically affect divided attention performance is practice. The effects of practice remain somewhat elusive, although they have at least been described in perceptual response mapping (Jordan \& Rabbitt, 1977), and in the learning of novel motor skills (Anshel, 1978). Practice effects seem to be questionable or absent in implicit memory tasks (Hashtroudi, Chrosniak, \& Schwartz, 1991), memory span capacity tasks (Taub, 1973), 
visual competition (Korteling, 1991), and memory search tasks (Strayer \& Kramer, 1994), and in tasks measuring interference susceptibility (Fisk, Cooper, Hertzog; \& Anderson Garlach, 1995). Hashtroudi and colleagues (1991) proposed that age deficits in skill learning are related to a deficit in perceptual (re)organization, a suggestion that was supported by Rogers and Fisk (1991). The suggestion of Rogers, Bertus, and Gulbert (1994) that practice may play an important role in determining age-related dual-task differences may not apply to all dual-task performance, as was evidenced by the 1986 study of McDowd (1986). Although absolute levells of performance in a perceptual-motor task improved reliably over sessions for both young and old subjects in their study, divided attention performance remained worse in old subjects than in young subjects.

A task that is affected by almost all aforementioned variables is the Paced Auditory Serial Addition Task (PASAT; Gronwall, 1977; Gronwall \& Sampson, 1974). In this task, a random series of digits from 1 to 9 is presented by means of a tape recorder or a computer soundboard. The task requires the subject to add pairs of digits such that the last digit heard is added to the preceding one, not to the sum of the previous addition. The PASAT reflects the capacity for divided attention and is sensitive to minor attention deficits after mild traumatic brain injury (TBI). Patients with postconcussional symptoms consistently perform well below control group averages immediately after injury or return to consciousness. (Gronwall \& Sampson; 1974; Stuss, Stethem, Hugenholtz, \& Richard, 1989). Most patients with mild TBI return to normal performance within 30 to 60 days after injury, although others continue to lag behind the performance level of their control group (Leininger, Gramling, Farrell, Kreutzer, \& Peck, 1990). Severe TBI may result in persistent reductions in information processing speed (Ponsford \& Kinsella, 1992; Stuss et al,; 1989). Based on an evaluation of how the PASAT performance was associated with performances on memory and attention tasks, Gronwall and Wrightson (1981) considered the PASAT to be very sensitive to deficits in information processing ability. Not surprisingly, performance levels on this speed-dependent task decline with age (Brittain, La Marche, Reeder, Roth, \& Boll, 1991). Roman, Edwall, Buchanan, and Patton (1991) showed that the effects of age on PASAT performance were most pronounced after 60 years. Roman and colleagues (1991) found no significant sex effects, although Brittain and colleagues (1991) found that sex had a small statistically significant effects, but they did not consider this to be clinically meaningful. Indexes of mental ability like education and intelligence, however, do affect performance. Years of education corfelated with PASAT performance (Stuss, Stethem, \& Poirier, 1987), as did intelligence in adult versions of the PASAT (Brittain et al., 1991; Roman et al, 1991) and with the CHISPASAT, a PASAT specifically designed for testing children (Dyche \& Johnison, 1991; Johnson, Roethig, \& Middleton, 1988). To study paced serial addition performance in middle-aged and old subjects, De Vries, Van Houte, Lindeboom, Van Eijk, and De Haan (1992) developed a version with longer interstimulus intervals between digits. They also found a negative correlation witti educational level and intelligence.

This study addressed several topics. In the first place, this study aimed detect the determinants of the decrease in divided attention in four age groups, using a self-paced version of the PASAT, which will be referred to as the SPASAT. This means that subsequent stimuli do not appear at fixed intervals, but are presented when a subject has finished the calculation proceriure. There are various reasons to use a self-paced serial addition task. Gronwall (1977) did not consider the PASAT to be suited for use with elderly subjects. Subjects of any age experience this task as very stressful: most people feel under great pressure and that they are 


\section{CHAPTER 6}

falling, even when they are doing well (Spreen \& Strauss, 1991; Stuss et al. 1989). The too fast pace for eldetly subjects futhermore leads to them making many errors, being frustrated, and giving up (Brittain et a1., 1991; Roman et al., 1991). Apart from measures of performance efficiency - number of errors and restarts - the SPASAT also provides information concerning the central processing time needed for simultaneous processing. Since older subjects tend to perform worse on speeded tasks like the PASAT (Brittain et al, 1991), we wanted to detect whether initial differences in speed might also be reflected in a self-paced task. The SPASAT furthermore requires subjects to make rather simple calculations, but initial differences in arithmetic ability might possibly allso affect age differences in speed, number of errors or restarts. Inter-individual differences in this ability were evaluated in the light of the general slowing that is usually found among elderly subjects in speeded tasks. Differences in memory capacity, and in particular the capacity to retrieve information from short-term memory, might also play a role in performance. Hartley (1986) suggested that working memory capacity might show an age-related decline, which is consistent with the general slowing explanation of cognitive aging. The SPASAT requires subjects to shift between different cognitive operations. After hearing a new digit, subjects have to keep it in memory, remove both the previous digit and the solution of the calculation from memory, and periorm a new calculation. If subjects have difficulty in shifting between these operations, we would expect this to be expressed in SPASAT outcome measures. Hasher and Zacks (1988) suggested that older subjects are more vulnerable to interfering or irrelevant information. If the necessary operations are not performed successfully (i.e., not all information connected to previous targets is erased from memory), the memory traces of previous operations interfere with current task performance. Finally, the suggestion of Rabbitt (1991) and of Van Rooij and Plomp (1991) that perceptual deficits might account fol a great deal of the decline in cognitive performance observed in the elderly was evaluated with regard to the quantitative and qualitative measures of SPASAT performance. We hypothesized that subjects whose performance on any of the determinants reviewed in this. paragraph was compromised would show an attenuation of changes in SPASAT performance that are related to age.

The second topic we studied was whether subjects of all ages benefitted from practice to the same extent. To study these effects, the SPASAT was administered at the beginning and at the end of an extensive netropsychological investigation. Gronwall (1977) and Stuss and colleagues (1987) reported increased performance after repeated test administrations. These practice effects may be due to purely attentional factors, but may also reflect strategy use (such as reheursal or skipping number), automatization of the simple addition process, or affective variables (such as frustration with the task). To study practice effects in more detail we not only compared the first and second SPASAT performance, but we also examined how task performance changed within a single administration. Salthouse and colleagues (1995) suggested that age has little influence on dual-task performance relative to single-task performance. Inferences about the divided attention performance of elderly people have mostly been based on speeded tasks. A sell-paced administration of the PASAT enabled us to study practice effects in the absence of these time constraints. As older subjects show an overall slowing of performance (Salthouse, 1985), we expected this also to be expressed in the RTs. Several studies suggest that older subject tend to trade speed for accuracy, and younger subject tend to trade accuracy for speed (Madden, 1985; Rush, Panek, \& Russell, 1987; Smith \& Brewer, 1995; Strayer, \& Kramer, 1994), although the presence of age-related strategy differences is highly controversial. The speed-accuracy trade-off function relates mean accuracy with mean $\mathrm{RT}$ for 
blocks of trials (Luce, 1986). In contrast with previous findings, Salthouse (1993a) found that although the absolute magnitude of the age differences was greatest for the slowest RTs in each individual's RT distribution, there was little or no independent age-related variance in the slowest RTs after controlling for the variance in the fastest RTs. Furthermore, the relation between RT and measures of motor speed, perceptual speed, working memory, and aceuracy in several cognitive tasks was of nearly the same magnitude when only the fastest responses were considered and when both fast and slow responses were considered. He therefore suggested that age-related slowing is associated with a shift (and expansion) in the entire RT distribution, and is not attributable to a selective influence on the individual's slowest responses. If a speed-accuracy trade-off plays a role in our study, we would expect older subjects to perform at equivalent levels as young subjects when the number of errors and restarts are considered. Since the time between the first and second administration of the SPASAT was devoted to neuropsychological testing, we expected the practice effects - if any--in elderly subjects to be compromised by a decrease in the processing resources available because these resources had been allocated to the other neuropsychological tests.

This study was conducted as part of a large cross-sectional research program into biological and psychological determinants of successful and pathological cognitive aging, named the Maastricht Aging Study (MAAS Jolles, Houx, Van Boxtel, \& Ponds, 1995). Subjects were recruited from a register of patients of general practices in the region of Mastricht (Metsemakers, Höppener, Knottnerus, Kocken, \& Limonard, 1992). This register contains all relevant past and current medical morbidity as documented by the general practitioners. Subjects with previous or current medical conditions with a known impact on cognitive and/or motor functions were excluded from the selection. Exclusion criteria were overt cerebrovascular disease, chronic neurological pathology (e.g., dementia, epilepsy, parkinsonism), mental retardation, chronic cardiovascular or pulmonary disease, and psychotropic drug use. All subjects were thus normal and healthy according to regular gerontological criteria. The subjects who were eligible for the study completed a postal questionnaire and participated in an extensive neuropsychological investigation. The standardized postal questionnaire yielded a subjective report on medical history and demographic information.

Subject sampling was stratified by 12 discontinuous age classes ( 25 years $\pm 1,30$ years $\pm 1,35$ years $\pm 1, \ldots, 80$ years \pm 1 ) and sex. For this study, subjects were assigned to one of four age categories, namely between 25 and 35 years (group 1), between 40 and 50 years (group 2), between 55 and 65 years (group 3), and between 70 and 80 years (group 4 ).

Two hundred and forty subjects were initially tested. Of these subjects, 22 were excluded because of invalid or unreliable results. In order to correct for oulliers or extremes, we excluded subjects with RTs, number of errors, or number of restarts of more than 2.5 standard deviations higher than the mean for the entire population on the first or second test administration. These were subjects who supposedly did not perform according to the test instructions. This meant that 2 subjects in group 1,4 subjects in group 2,8 subjects in group 3 , 
and 8 subjects in group 4 were deleted. The characteristics of the remaining 196 subjects whose data were used for analysis are summarized in Table 6.1.

Tablic 6.1

Demographic Characteristics of Normal, Healthy Subjects $(\mathrm{N}=196)$

\begin{tabular}{|c|c|c|c|c|c|c|c|c|c|}
\hline \multirow[b]{2}{*}{ Subjects } & \multicolumn{2}{|c|}{$N$} & \multicolumn{2}{|c|}{ Age } & \multicolumn{2}{|c|}{ Hearing Loss } & \multicolumn{2}{|c|}{ Intelligence } & \multirow{2}{*}{$\frac{\operatorname{Sex}(\mathrm{M} / \mathrm{F})}{\%}$} \\
\hline & w & (\%) & $M$ & $S D$ & $M$ & $S D$ & $M$ & $3 D$ & \\
\hline All & 196 & $(100)$ & 49.62 & 16.76 & 70.58 & 46.20 & 114.39 & 12.42 & $52 / 48$ \\
\hline $25-35$ & 56 & $(29)$ & 29.82 & 4.42 & 32.57 & 22.35 & 114.05 & 10.72 & $48 / 52$ \\
\hline $40-50$ & 57 & (29) & 44.58 & 4.07 & 58.51 & 29.62 & 113.26 & 12.90 & $53 / 47$ \\
\hline $55-65$ & 45 & (23) & 59.71 & 4.31 & 86.80 & 37.62 & 112.33 & 14.10 & $56 / 44$ \\
\hline $70-80$ & 38 & $(20)$ & 74.42 & 4.00 & 125.50 & 40.80 & 119.00 & 11.19 & $50 / 50$ \\
\hline
\end{tabular}

One-way analysis of variance did not show there to be significant differences between the IQ scores of the groups $(F(3,196)=2.37$, n.s. $)$. Duncan's multiple range test showed all groups to differ in hearing threshold (DSHL) at the 0.05 level $(F(3,196)=68.86, p<.001)$, with older subjects having a bigher hearing threshold.

\section{$6.2 .2 \quad$ Measures}

The SPASAT consisted of a digitized voice recording of digits which were presented aurally by means of a microcomputer soundboard and a loudspeaker. Sound level was adjusted until the subject could repeat a list of randomly presented numbers. Sixty-one single-digit numbers were presented in a pseudo-random order. This meant that two consecutive stimuli were never the same. The subject was to add successive digits in pairs and immediately give the answer aloud before the next digit was presented. For example, given the sequence $2,4,1,6$, the subject had to add $2+4$ and reply 6 , then add $4+1$ and reply 5 , then add $1+6$ and reply 7 . The sequence of digits was such that no answer exceeded 18. As soon as the subject gave the solution, the experimenter pressed a yes/no button to indicate whether the answer was correct or not. Only RT's of the total number of 60 possible correct solutions were calculated by a microcomputer. If the subject forgot the last digit, two new pairs of digits were presented. This was also the case when the subject did not respond within 5000 milliseconds, after which we considered reactions to reflect processes other than the simple addition of digits. These conditions were registered as "restarts". The PASAT differs from our task in that the time of presentation of subsequent digits depends on the time the subjects take to give the solution. In the PASAT, digits are presented in four trials with $2.4-, 2.0-, 1.6-$, and 1.2-second intervals for the respective trials. Prior to the beginning of the experiment, a demonstration with written digits was given until the subjects understood what they had to do. Subsequently, subjects participated in a training session consisting of 10 digits spoken by the experimenter. This session lasted until the subjects stated that they fully understood the test procedure.

Simple RT was measured with the Motor Choice Reaction Test (Houx \& Jolles, 1993), by which RTs are studied as a function of task complexity. In this task, the subject was requested 
to hold down, with the index finger, a red home button. When a target button lit we, the subject had to press this button with the same finger. The home button had to be held down immediately after the target button was pressed until the next button lit up. The measwe used for analysis was the motor initiation response time.

Intelligence was estimated with four subtasks of the Groningen Intelligence Test (GT; Luteinn \& Van der Ploeg, 1983), which is a test of general intelligence in the Netherlands. There is general agreement on which subtasks should be used to arrive at the best possible approximation of a full-scale IQ: "Arithmetics" involves the correct completion of as manyy sums (addition) as possible in 1 minute; in the "Vocabulary" subtask the subject is asked to indicate which of five alternative words is exactly synonymous with a given word; "Mental rotation" requires the subject to indicate which two-dimensional shapes from a larger set are needed to exactly fill up a given space on the test page, which means that subjects have to mentally rotate each of the shapes, as they are, as a rule, not presented in the proper orientation; "Analogies" can be regarded as a multiple choice version of the well-known "Similarities" subtask of the WAIS: the task is to indicate which of five alternatives is related in the same way to a given word as two words in an example. The measure used for the regression analysis is the Arithmetics score. The score on all four subtasks is used as covariate in the analysis of variance.

Memory performance was measured by the "Groningen Fifteen Words Test" (Brand \& Jolles, 1985; Deelman, Brouwer, Van Zomeren, \& Saan, 1980), which is an improved version of a test originally devised by Rey (1964). Briefly, 15 words are presented, one after another, separated by short time intervals. The subject is asked to recall as many words as he or she can, in his or her own order. This procedure is repeated five times, so that a learning curve can be plotted. After 20 minutes, delayed recall and recognition are tested. The computerized test was given visually. The number of words recalled at the first trial is used as a measure of immediate memory storage.

The Concept Shifting Test (CST; Houx \& Jolles, 1994) is an adaptation of the Trail Making Test, which is a test of visual conceptual and visuomotor tracking (Lezak, 1995). The CST consists of three subtasks. In each subtask, the subject has to mark small circles with digits or letters in ascending order on an A4 size sheet of paper. The subject is requested to do this as quickly as possible. On each test sheet, 16 small circles are grouped in a larger circle. In the smaller circles, the test items (numbers (CSTA), letters (CSTB), or both (CSTC)) appear in a fixed random order. The subjects are requested to cross out the items in the right order, in the same way as in the TMT. The relative contribution of the need to shift between two concepts to the total time needed for subtask $\mathrm{C}$ (percentage) is computed with the formula:

$$
\frac{\text { CSTC }-.5(\text { CSTA CSTB })}{.5(\text { CSTA CSTB })} * 100 \%
$$

where CSTA, CSTB, and CSTC denote the total time taken to complete the test part.

Interference susceptibility was estimated with the Stroop Color Word Test (SCWT; Lezak, 1995), which is a perceptual interference task and consists of three cards. The test examines the speed at which color names are read (SCWT-I) and the speed at which colored patches are named (SCWT-II). SCWT-III involves color names again, but the printing ink is different from 
the color mane. To have a measure of interference susceptibility irrespective of age, interference susceptibility is expressed by the percentage of extra time needed for SCWT-II, relative to the average of the first wo cards:

$$
\frac{\text { SCWTII }-5(\text { SCWTI }+ \text { SCWTII) }}{5(\text { SCWT }+ \text { SCWTI })} * 100 \%
$$

Hearing loss was assessed at four different frequencies that are relewant to speech, using a tone addiogram. Each ear was tested separately at frequencies of $500,1,000,2,000$, and $4,000 \mathrm{~Hz}$ in steps of $10 \mathrm{~dB}$ to determine the pure tone detection threshold. The American Academy of Otolaryngology Committee on Hearing and Equilibrium (1979) provided normative data as to which degree of hearing loss is associated with disability. Since we studied subjects in the age range of 25-80 years, we expected the disability to be restricted to the old middle-aged and the old group. This in fact turned out to be true: the number of subjects with a hearing handicap for groups 1,2,3, and 4 was $2,9,21$, and 34 , respectively. To detect how a minor - but not invalidating- hearing impaiment might affect PASAT performance, we used the degree of $\mathrm{dB}$ loss for the ear that was compromised as index measure. If both ears were affected and monaural hearing impaiments were not the same, the following fomula was applied (American Academy of Otolaryngology Conmittee on Hearing and Equilibrium, 1979):

$$
\frac{(5 * \text { dB loss beter ear })+(\mathrm{dB} \text { loss poorer ear })}{6}
$$

\subsubsection{Data Analysis}

The study involved subject repeated measures, each subject being assessed with the SPASAT at two times, separated by approximately 2.5 hours, during which the other neuropsychological tests were administered. Overall time-on-task effects associated with aging were analyzed by using repeated measures analysis of variance with age (four levels) and time (two levels) entered as variables. Since intelligence, corrected for age, negatively correlated with the mean response time $(-29, p<.001)$, with the mean number of errors $(-.14, p<.05)$, and with the mean number of restarts $(-.38, p<.001)$ on the first SPASAT administration,we used intelligence as a covariate in our analysis. The level of significance was set at $p<.05$, to Indicate which test variables were suitable for subsequent post-hoc analysis. Post-hoc analyses of age effects on performance on the first and second test administrations were performed by analysis of covariance and were also corrected for intelligence $(p<.05)$. Time-on-task effects within age groups at either administration were analyzed by using t-tests for paired samples. The possibility that speed-accuracy strategies were used was examined by partial correlations between RT and number of errors, controlling for age.

In order to decide which intra-individual factors contribute to performance, we entered simple RT, arithmetic ability, primary memory capacity, concept shifting ability, interference susceptibility, degree of hearing loss, and age in a stepwise hierarchical multiple regression model. Dependent variables were the mean SPASAT RT, number of errors, and number of restarts on the first administration. Characteristics of these predictors are summarized in Table 6.2. Descriptions of age and hearing loss can be found in Table 6.1. The following variables entered in the regression analysis decreased with age: simple RT $(F(3,196)=14.62, p<$ $.001)$, primary memory $(F(3,196)=26.25, p<.001)$, concept $\operatorname{shifting}(F(3,191)=5.50, p$ $<.01)$, and interference susceptibility $(F(3,191)=12.12, p<.001)$. Arithmetic ability did not 
appear to be age-dependent $(F(3,196)=1.76, \mathrm{n} . \mathrm{s}$.$) .$

Table 6.2

Characteristics of variables for regression analysis $(\mathrm{N}=196)$

\begin{tabular}{|c|c|c|c|c|c|c|c|c|c|c|}
\hline \multirow[t]{2}{*}{ Age (yrs) } & \multicolumn{2}{|c|}{ Simple RT } & \multicolumn{2}{|c|}{ Arithmetics } & \multicolumn{2}{|c|}{ Memory } & \multicolumn{2}{|c|}{$\begin{array}{l}\text { Concept } \\
\text { Strifting }\end{array}$} & \multicolumn{2}{|c|}{$\begin{array}{c}\text { Interfenence } \\
\text { Susceptibility }\end{array}$} \\
\hline & $M$ & $S D$ & $M$ & $S D$ & $M$ & $S D$ & $M$ & SD & $M$ & $S D$ \\
\hline All & 328.67 & 56.06 & 12.33 & 4.03 & 5.49 & 1.70 & 52.17 & 36.66 & 90.45 & 31.66 \\
\hline 25.40 & 311.63 & 48.54 & 11.66 & 3.43 & 6.54 & 1.45 & 42.86 & 31.69 & 77.20 & 26.29 \\
\hline $40-50$ & 314.58 & 36.77 & 13,25 & 4.70 & 5.77 & 1.34 & 50.44 & 32.40 & 82.64 & 28.59 \\
\hline $55-65$ & 327.47 & 40.92 & 12.44 & 3.85 & 5.16 & 1.66 & 48.77 & 29.36 & 99.03 & 27.87 \\
\hline $70-80$ & 376.32 & 77.110 & 11.79 & 3.81 & 3.92 & 1.30 & 72.71 & 49.14 & 111.99 & 34.09 \\
\hline
\end{tabular}

Note: Descriptions of age and hearing loss are summarized in Table 6.1

\section{$6.3 \quad$ Results}

\section{Response time}

Comparison of the speed measures depicted in Figure 6.1 showed that older participants were significantly slower than younger subjects on both the first and the second SPASAT administrations $(F(3,196)=14.77, p<.001$ and $F(1,196)=23.79, p<.001$, respectively $)$. From age 40 onwards, the decline in speed appeared to be described by a linear function. As expected, the subjects performed more slowly as they got older. Repeated measures analysis of variance showed significant overall effects of age $(F(3,196)=21.36, p<.001)$ and of time $(F$ $(1,196)=14.98, p<.001)$ when the two administrations were evaluated together. The small but significant Age $\times$ Time interaction $(F(3,196)=2.95, p<.05)$ could be attributed to the fact that subjects of 25 to 35 and 40 to 50 years were faster at the second administration $(t=4.42$, $d f=55, p<.001$ and $r=4.48, d f=56, p<.001$, respectively). Performance of the two older groups did mot differ at the .05 level, and it can thus be conclided that subjects older than 55 did not benefit from practice in terms of speed of performance.

Age differences in speed of performance within the first SPASAT administration were studied by performing repeated measures analyses of variance on the first and second halves of this test after correction for intelligence. The first half of the first administration showed a linear increase in speed associated with age (Age: $F(3,196)=14.98, p<.001$ ), whereas in the second thalf subjects tended to become slower (Time: $F(1,196)=10.19, p<.01$ ). This effect was most. pronounced in the youngest group, whose speed of performance dropped dramatically. The decrease in performance in this group $(t=-4.52, d f=55, p<.001)$ gave rise to an Age $x$ Time interaction (Age X Time: $F(3,196)=3.00, p<.05)$. Inspection of speed on the first and second halves of the second administration showed rather different results. As was the case in the first administration, older subjects tended to be slower (Age: $(F(3,196)=23.90$, $p<$ .001). None of the subjects, however, showed any difference in speed on the first or second half of the second administration, which was demonstrated by the lack of Time effects or Age $x$ Time interactions (i.e., performance remained stable over time). 
Figure 6.1 (left): Mean SPASAT respontse time of subjects aged 25-80 years on the first (SPASAT-1) and second administration (SPASAT-2). Vertical bars denote SEMs.

Figure 6.2 (right): Mean number of SPASAT errors ( $+5 E M s$ ) of subjects aged 25-80 years on the first (SPASAT-1) and second administration (SPASAT-2).
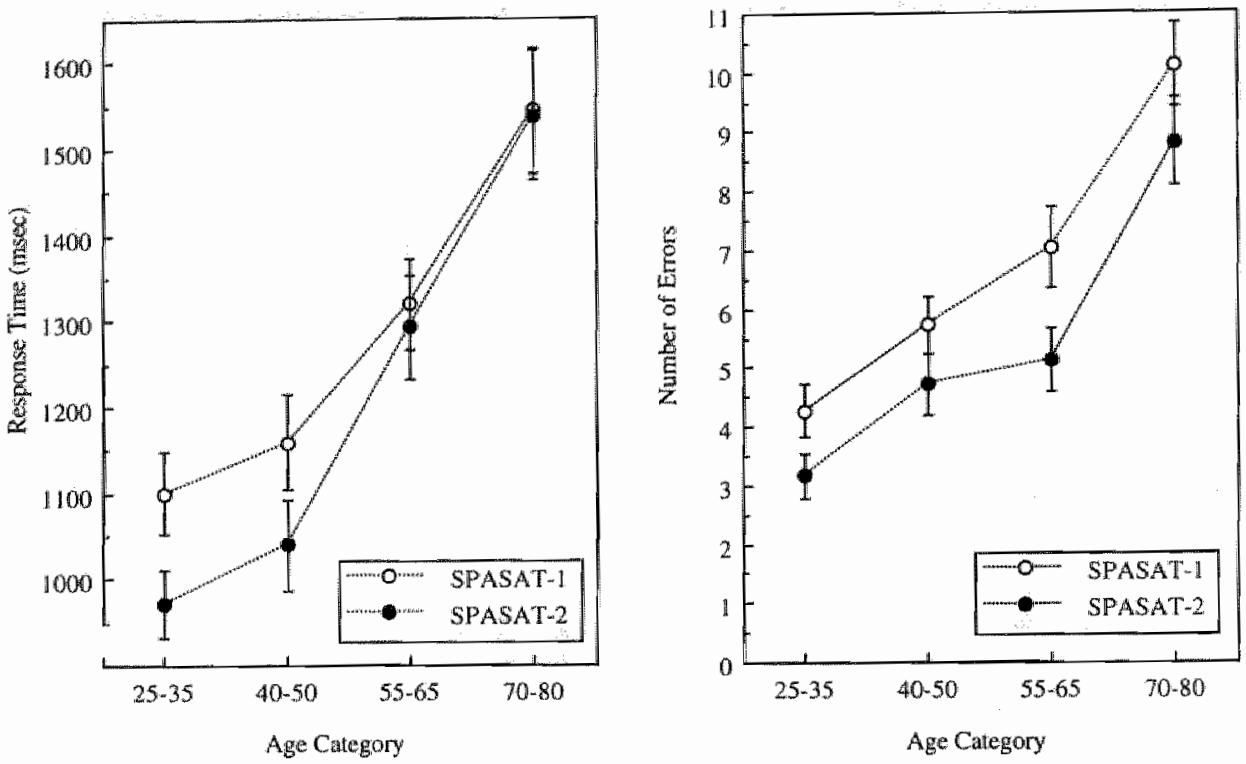

Errors

A consistent finding was that not only RTs, but also the number of errors tended to increase as a function of age (Figure $6.2: F(3,196)=24.72, p<.001)$. The $F$-values expressing this effect appeared to be rather stable over time, and thus were similar for the first $(F(3,196)=$ $18.49, p<.001)$ and second SPASAT administrations $(F(3,196)=19.71, p<.001)$. Practice improved the performance of all subjects (Time: $F(1,196)=24.46, p<.001$ ) except for that of subjects in the oldest group, whose performance did not significantly increase (group 1, 2 , and $3: t=2.98, d f=55, p<.01, t=2.02, d f=56, p<.05$, and $t=3.26, d f=44, p<.01$, respectively). Age $x$ Time interactions could not be demonstrated.

The first and second halves of the first administration showed an age-dependent increase in the number of errors (Age: $F(3,196)=18.48, p<.001$ ), as well as an evident increase in the number of errors towards the end of the first administration (Time: $F(1,196)=15.64, p<$ $.001)$, although this only proved to be significant for groups 2 and $3(t=-2.45, d f=56, p<$ .05 and $t=-2.94, d f=44, p<.01$, respectively). An Age $x$ Time interaction was not found. This interaction effect was also lacking for the second administration of the task. With the second administration, subjects also tended to perform worse in the second half than in the first half (Time: $F(1,196)=9.88, p<.01)$, and age had a clear (Age: $F(3,196)=19.71, p<$ .001). Although performance declined, this time the younger age group tended to have a poorer performance in the second half (group 1 and $2: t=-2.77, d f=55, p<.01$ and $t=-3.28, d f=$ 
$56, p<.01$, respectively).

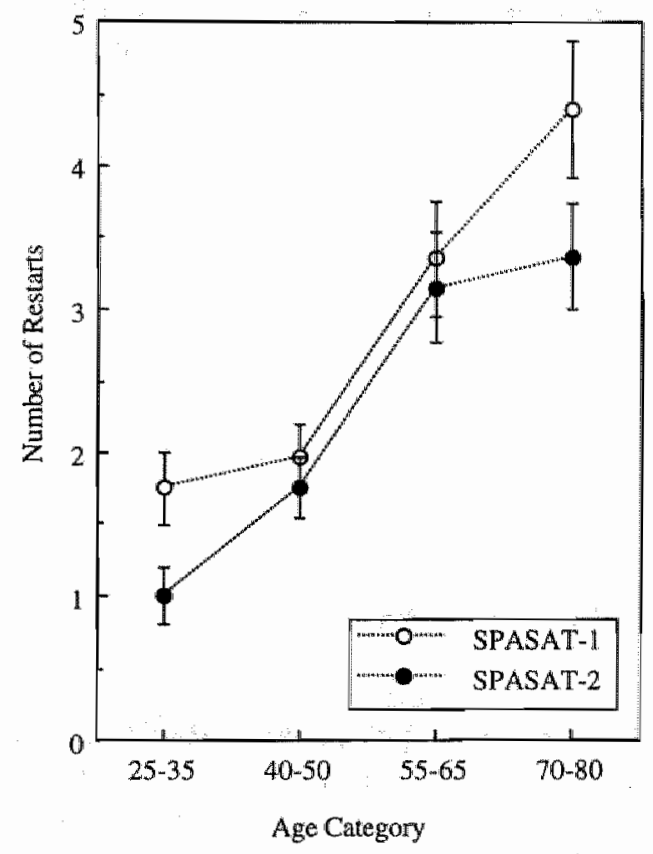

\section{Restarts}

Age affected the number of restarts in both the first and second administrations (Figure 6.3: $F$ $(3,196)=18.61, p<.001$ and $F(\mathbb{1}, 196)=19.95, p<.001$, respectively). Repeated measures analysis of variance proved this to be an overall effect that was stable across administrations (Age: $F(3,196)=24.54, p<.001)$.

The repeated administration of the SPASAT improved performance (Time: $F(1,196)=14.94$, $p<.001$ ), although this improvement was significant in the youngest and oldest groups only (group 1 and 4: $t=3.56, d f=55, p<.001$ and $t=2.80, d f=37, p<.01$, respectively). Apart from the already recognized age effects (Age: $F(3,196)=18.61, p<.001$ ), tnalysis of time-on-task effects in the first administration showed that the two youngest groups tended to have more restarts in the second half, whereas the oldest group tendended to improve performance, thus yielding a near-significant Age $\mathrm{x}$ Time interaction (Age $\mathrm{x}$ Time: $F(3,196)=$ 18.61, $p=.054)$. This trend was only significant for groups 1 and 2 , however $(t=-2.70, d f=$ $55, p<.01$ and $t=-2.13, d f=56, p<.04$, respectively). The second administration only showed a strong effect of age only, with younger subjects performing better (Age: $F(3,196)=$ $19.95, p<.001)$. There was no statistically significant difference between the number of restarts in the first and second halves of hte second test administration in any age group. 


\section{$118+$ CHAPTHR 6}

\section{Speed accuracy}

In order to study whether strategles might have contributed to differences in performance as a function of age, we calculated the correlation between SPASAT RT and the number of errors and restarts of the first and second administration. The results of the analysis are summarized in Table 6.3. It is noteworthy that on the first administration faster RTs were associated with an increase in the number of errors in the youngest group only. A faster RT in the SPASAT tended. to correlate better with an increase in the number of restarts. Analysis of correlations between RT and number of errors or number of restarts suggested that faster RTs in the old group did not lead to more errors, but did lead to an increase in restarts.

Table 6.3

Partial Correlation Coefficients Controlling for Age

\begin{tabular}{|c|c|c|c|c|c|c|c|c|c|c|c|}
\hline & Grousp & $\mathbb{E r} r-1$ & Sign. & Resil 1 & Sign. & & Group & $\mathrm{Err}-2$ & Sign. & $\operatorname{Rest} 2$ & Sign. \\
\hline \multirow[t]{4}{*}{ RT-I } & 25.35 & .2821 & $*$ & .3358 & *k & $\mathrm{RT}-2$ & $25-35$ & .3518 & $3 *$ & 2993 & * \\
\hline & $40-50$ & .191 & & .4312 & 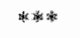 & & $40-50$ & .2820 & * & .4006 & 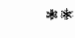 \\
\hline & $60 \times 70$ & -.0357 & & .457 .4 & * 和 & & $60-70$ & .2810 & 畨 & .2046 & \\
\hline & 70.80 & 050 & & .5544 & 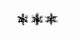 & & $70-80$ & .1310 & & .5166 & $* *$ \\
\hline
\end{tabular}

Note. RT-1 and RT-2 denote RTs on first and second administration, respectively; Err-1 and Enr-2 denote

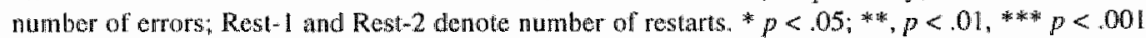

\section{Regression Analysis}

The results of the stepwise hierarchical multiple regression model are summarized in Table 6.4 . An increase in arithmetic ability and primary memory capacity tended to be associated with a better performance, whereas an increase in the values of the other predictors compromised SPASAT performance. Simple $\mathbb{R T}$, arithmetic ability, primary memory capacity, interference susceptibility, and degree of hearing loss significantly contributed to the change in $R 2$, accounting for $28.6 \%$ of the total explained variance in the SPASAT RT. After age was entered at the last step, this predictor stil] explained $5.3 \%$ of the total variance. The inclusion of the concept stufting ability, however, did not lead to a significant change in $R 2$.

With regard to the number of errors, initial differences in simple RT, primary memory capacity, concept shifting ability, degree of hearing loss, and age explained $26.6 \%$ of the total variance. Arithmetic ability and interference susceptibility did not appear to have a significant impact on this performance measure. As far as the number of restarts are concerned, after the initial differences in RT, age brought about the largest change in $R 2$ and yielded $8.5 \%$ of a total of $30.6 \%$ that could be explained by the listed predictors. The changes in $R 2$ associated with the ability to shift between concepts and hearing loss were not statistically significant, suggesting that performance in terms of number of restarts not affected by changes in perceptual acuity. 
Table 6.4

Stepwise hierarchical multiple regression analysis of SPASAT performance

\begin{tabular}{|c|c|c|c|c|c|c|}
\hline Variable & Predictors & Beta & to $R 2$ & $F$ & $d f$ & $p$ \\
\hline \multirow[t]{7}{*}{$\mathrm{RT}$} & Simple RT & .246 & .061 & 12.21 & $(1.190)$ & .001 \\
\hline & Arithmetics & -.378 & .141 & 33.23 & . & .000 \\
\hline & Menory & -.181 & .030 & 7.26 & . & .008 \\
\hline & Concept Shifting & .027 & .001 & 17 & & .684 \\
\hline & Interference Susceptibility & .150 & .020 & 4.93 & . & .028 \\
\hline & Hearing Loss & .222 & .053 & 8.41 & 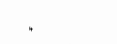 & .004 \\
\hline & Age & .388 & .053 & 14.69 & $(7,184)$ & .000 \\
\hline \multirow[t]{7}{*}{ Errors } & Simple RT & .276 & .076 & 15.62 & $(1,190)$ & .000 \\
\hline & Arithmetics & -.107 & .011 & 2.35 & 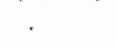 & .127 \\
\hline & Memory & -.209 & .040 & 8.57 & . & .004 \\
\hline & Concept Shifting & .182 & .032 & 7.03 & . & .009 \\
\hline & Interference Susceptibility & .131 & .015 & 3.44 & . & .065 \\
\hline & Hearing Loss & .322 & .069 & 16.77 & . & .000 \\
\hline & Age & .254 & .023 & 5.70 & $(7,184)$ & .018 \\
\hline \multirow[t]{7}{*}{ Restarts } & Simple RT & .317 & .100 & 21.11 & $(1.190)$ & .000 \\
\hline & Arithmetics & -180 & .032 & 6.95 & . & .009 \\
\hline & Memory & $\cdots .221$ & .045 & 10.13 & " & .002 \\
\hline & Concept Shifting & .082 & .006 & 1.47 &. & .228 \\
\hline & Interference Susceptibility & .205 & .037 & 8.82 & . & .003 \\
\hline & Hearing Loss & -.045 & .001 & .32 & . & .571 \\
\hline & Age & .489 & .085 & 22.32 & $(7,184)$ & .000 \\
\hline
\end{tabular}

\subsection{Discussion}

The first purpose of this study was to determine which factors are predictive of the performance in a non-speeded divided attention task. The second purpose of this study was to examine whether repeated administration of the test benefitted old and young subjects to the same extent. A related issue had to do with the uniformity of age-related slowing; that is, can qualitative performance, expressed in terms of errors and restarts, be explained by differences in strategies? (e.g., speed-accuracy trade-off).

Mean RTs for older individuals were slower than those for the younger in all conditions. With respect to this general phenomenon, Cerella (1991) concluded that aging is characterized by a generalized slowing of the central nervous system that uniformly affects all stages of information processing. The studies of Allen, Ashcraft, and Weber (1992) and Geary and Wiley (1991), however, suggest that age-related slowing does not generalize to lasks requiring simple mental arithmetic, which is one of the main operations involved in SPASAT performance. In contrast to the study of Roman and colleagues (1991), who found the decrease in PASAT performance to be most pronounced after age 60 , we found no overall evidence for a discontinuity in the function describing the increase of the number of errors or restarts at a any particular age. The fact that the original PASAT is considered to be too stressful for older 


\section{CHAPTER 6}

subjects, which may lead to many errors (Spreen \& Strauss, 1991; Stuss et al., 1989), may in part explain this discrepancy. After initial differences in simple $R T$, arithmetic ability, primary memory capacity, concept shifting ability, interference susceptibility, and degree of hearing loss were entered in a stepwise hierarchical multiple regression model, age could still explain a significant proportion of the total variance on SPASAT RT, errors, and restarts. This suggests that divided attention capacity declines with age, both in tems of speed and in terms of efficiency. It is noteworthy, however, that the various predictors of SPASAT performance appeared to have changing weights, depending on the outcome measure considered. Differences in the ability to shift between concepts, for instance, did not significantly affect SPASAT RT, and arithmetic ability and interference susceptibility did not affect the number of errors. The number of restants furthermore did not appear to be affected by differences in concept shifting abilily and degree of hearing loss. This suggests that the various SPASAT outcome measures differ in their dependence on peripheral. or central processing. The percentage of the variance in SPASAT RT and errors explained by hearing loss is considered inidcative of the "knock-on effects" hypothesis of Rabbitt (1991) and Van Rooij and Plomp (1991), who suggested that perceptual loss at peripheral levels may affect processing at central \evels, even though subjects indicated that they had no difficulty in perceiving the auditory presented digits. Stankov (1986) demonstrated that auditory functions are independent and separate from crystallized or fluid intelligence, and thus explain a unique percentage of the variance in SPASAT performance.

The beta weights associated with the primary memory capacity in Table 6.4 show that SPASAT performance also depends on primary memory capacity, regardless of the measures that are taken as an index of SPASAT performance. Age-related decreases in many aspects of cognitive performance have been attributed to a smaller storage capacity of working memory with increasing age (Campbell \& Chamess, 1990; Light, Zelinski, \& Moore, 1982; Salthouse, 1992; Salthouse, 1993b; Salthouse \& Babcock, 199I). Research in this area has been based primarily on a two-concept view of working memory (see Anderson, 1990) that emphasizes speed of activation and strength of connections in a propositional network. An alternative view of working memory, that of Baddeley (1986), is framed in terms of discrete and relatively independent subsystems. These subsystems have been suggested to include a phonological loop, a visual sketch pad, and a modality-free central executive. Anderson's 1990 model proposes that information is stored within a propositional network in which interrelationships are represented by paths. In the process of recall, the propositional network is activated. Because this activation takes time, the speed with which it occurs determines the efficiency of the system. Speed will depend on the richness of connections within the network, and it is to be expected that there are individual differences in the rate at which information is activated. The central cognitive operation underlying simple arithmetic problem solving is the retrieval of facts from long-term memory (Allen et al., 1992; Geary \& Willey, 1991). In the study of Geary and Wiley (1991), old subjects reported using retrieval to solve $98 \%$ of the addition problems with which they were presented, whereas young subjects reported using retrieval in only $88 \%$ of the trials. The decrease in primary memory capacity and the greater dependence on memory retrieval with increasing age in our study might be a plausible explanation for the behavioral slowing seen in the elderly subjects. The slower activation of the network associated with memory retrieval might be the underlying neuronal mechanism. When neuronal connections are lost with age, the information has to be processed along alternative nodes or paths, which consequently decreases the response speed (Cerella, 1990). Aging, however, is not necessarily associated with an irreversible decrease in synaptic connections, as was demonstrated by Swaab 
(1991) and by Swaab, Hofman, Lucassen, Salehi, and Uylings (1994). Applying the Baddeley (1986) model to our SPASAT study, we propose that the last presented digit is stored in the phonological loop in which it is rehearsed for future use, presurnably under the control of the central executive. A decline in executive function would cause older subjects to have an inferior performance compared to younger subjects. Fisk and Warr (1996), however, found no evidence for a decline in central executive functioning with age, but stated that the decline in performance associated with aging is primarily attributable to a general slowing of the rate at which information is activated within the working memory system. This is in accordance with the findings of Salthouse (1994), who stated that although increased age is associated with lower scores on measures of working memory functioning, many of the age-related influences appear to be mediated by a slower speed of processing. Furthermore, recent studies indicate that slower processing primarily influences the time required to achieve a stable encoding of information rather than the rate at which information is lost across time or subsequent processing. Basic processing speed in our study also turned out to have a greater value in predicting SPASAT performance than primary memory capacity.

The ability to inhibit interfering or redundant information decreases as a function of age (Houx, Vreeling, \& Jolles, 1993; Klein, Ponds, Houx, \& Jolles, 1997; MacLeod, 1991) and has been demonstrated to affect RTs and number of restarts in our study. The SPASAT not only requires subjects to attend to environmental information, but also demands the active suppression of internal information, most notably the previous sum in working memory storage. Reporting the previous sum is the most common error in the task, since subjects tend to recall it and add it to the next number when their performance becomes worse. Hasher and coworkers (1991) and Hasher and Zacks (1988) considered inhibitory control as a central mechanism determining the content of working memory and consequently influencing cognitive functions. The decreased ability to employ inhibitory mechanisms may be another plausible explanation of the age-related decline in SPASAT performance. Deary and coworkers (1994) found a significant redistribution of a function-related bloodflow marker in the right anterior cingulate and left posterior cingulate areas during PASAT activation. The anterior cingulate is likely to be involved in tasks requiring supervisory control (Duncan, Burgess, \& Emslie, 1995; Shallice, 1988; Shallice \& Burgess, 1991a; Shallice \& Burgess, 1991b). Raichle and coworkers (1994) indeed showed the anterior cingulate to be active when the Supervisory Attentional System (SAS) is necessary for appropriate behavior, as it is in the Norman-Shallice model (Norman \& Shallice, 1986). The functional decline of inhibitory mechanisms in the elderly, which give rise to the presence of redundant and distracting information in working memory, might have compromised the optimal functioning of SAS, since this control system depends on the strategies active in working memory.

Practice effects were examined by contrasting performance during the first and second SPASAT administrations. As might be expected from the literature (Gronwall, 1977; Stuss et al., 1987), we found performance to increase with practice, but not for all groups. As far as RT was concerned, we only found the performance of the two younger groups to increase over time. The number of errors did not decrease in the oldest group, whereas the number of restarts decreased in the youngest and the oldest groups, but - as will be reviewed-presumably for different reasons. In order to detect whether performance was stable over time within a single administration, we compared speed and efficiency in the first and second halves of either administration. On the first administration the youngest group started relatively fast and showed a sharp decrease in speed on the second half. This phenomenon is in line with the findings of 
Steyvers and Gallatd (1993), who noticed that when subjects are not rewarded for their performance, they seem to start the task by doing their utmost, and thus spend resources early during the task. The same authors furthermore propose that the influence of time-on-task is not due to a lack of arousal, but is due to a teallocation of resources, which they suggest to be more successful in younger subjects. In our study, however, we found no evidence for a more efficient allocation of ressources in the youngest group, since they slowed down in the second half of the first administration and furthermore showed an increase in the number of restarts, but not in errors. The oldest group was slower than any other group. These subjects did not show a change in the speed of performance, but they also did not show an increase in the number of errors and even tended to have fewer errors in the second half of the test. This suggests that although they might be slower, older subjects are more capable of estimating the appropriate speed at which they can perform a cognitive operation successfully. Older subjects thus appear to be more cautious when confronted with new or unfamiliar situations. Rush and coworkers (1987) and Rush, Panek, and Russell (1990) proposed that cautiousness in older subjects is manifested more in terms of accuracy than in terms of response speed, and that the level of cautiousness increases with increasing age. They suggested that more cautious older adults can be expected to have significantly longer RTs and to commit significantly fewer errors. In our study, however, besides longer RTs; we found age to be associated with an increase in errors and restarts. This might be due to the fact that although older subjects probably process distracting information in a more cautious way, they still fail to employ inhibitory mechanisms to suppress distracting stimuli. This notion is supported by Klein and colleagues (1997), who showed that older subjects tended to start relatively slower in new or unfamiliar situations, as is the case in the Stroop interference condition, but at the same time still made more errors and spontaneous corrections than younger participants. Kane, Hasher, Stolzfus, Zacks, and Connelly (1994) compensated for the age-related slowing of processing and still found that older subjects could not benefit from inhibitory mechanisms when confronted with distracting stimuli. The results of the present study and the study by Klein and colleagues (1997) strongly suggest that elderly subjects are more susceptible to both external and internal interference.

When the SPASAT was administered a second time, there were no differences in speed on the First and second halves of the test, nor were any differences discerned in the number of restarts in any age group. The two younger groups, however, showed an increase in the number of errors on the second half of the second test administration. This suggests that although performance improves from the first to the second test administration in younger subjects, efficiency over time appears to fluctuate more than in older subjects. Our study does not support the notion that shorter RTs might lead to inferior performance specifically in the elderly (Table 6.3). In fact, we found that faster RTs on the first test administration were associated with an increase in errors only in the youngest group. On the second administration, there also was no correlation between RT and number of errors in the oldest group. This can be viewed as evidence for the suggestion that older subjects appear to be more able to monitor their performance levels as far as errors are concerned and adjust their speed to obtain optimal performance levels. Shorter RTs appear to be much more associated with the number of restarts, suggesting that error proneness in the elderly is less speed dependent than the mechanisms that give rise to restarts in the SPASAT.

Apart from an explanation in terms of cautiousness, it is also possible that the lack of practice effects on RTs in the elderly is related to the fact that elderly subjects are less able to develop 
new autonatic processes or to adapt existing automatic processes, as was suggested in a Stroop study by Dulaney and Rogers (1994). The multiple task demands of the SPASAT might have prevented the two older groups from adapting existing automatic processes, as is the case in the addition of single digits, which primarily depends on retrieval from memory. The youngest subjects became slower in the second part of the first test administration because they could only sustain active and efficient information processing for a limited time. Described in terms of processing strategies (Shiffrin \& Schneider, 1977), the young group completed the first half rapidly because of a controlled processing strategy requiring extra mental effort and the availability of more cognitive processing resources (Salthouse, 1988a; Salthouse, 1988b). When the task duration increased, these resources may have become exhausted, which gave rise to a decreased speed of performance.

In conclusion, the present study stresses the notion that slowing factors cannot be accurately estimated unless the cognitive operations involved in a task are taken into account (Laver \& Burke, 1993; Rogers \& Fisk, 1990). Various predictors of SPASAT performance have been identified that vary in weight depending on the outcome measure that is used to evaluate divided attention performance as a function of age. SPASAT performance definitely decreases with increasing age, but elderly subjects appear to be more able to monitor their own cognitive performance, leading to a more stable performance over time. The use of the SPASAT is considered to be less stressful than the PASAT, and therefore subjects -in particular the elderly - are less likely to give up. The use of a self-paced version of the PASAT furthermore allows us to study performance across a wide age range, without the necessity to adjust interstimulus intervals for subsequent age groups. The ease of administration and the cognitive variables that can be measured make the SPASAT a suitable instrument for measuring attentional functions in large-scale studies of cognitive aging.

\section{5}

\section{References}

Allen, P. A. Asheraft, M. H., \& Weber, T. A. (1992). On mental multiplication and ange. Prychology and Aging. 7(4), 536-545.

American Academy of Otolarymgology Committee on Hearing and Equilibrium (1979). Guide for the evaluation of hearing thandicap. Journal of the American Medical Association, 24l(19), 2055-2059.

Anderson, J. R. (1990). Cognitive Psychology and its Implications. New York: Freeman.

Anshel. M. H. (1978). Effect of aging on acquisition and short-tern retention of a motor skill: Perceptual and Motor Skills, 47(3), 993-994.

Baddeley, A. (1986). Working Menory. London: Oxford Uniwersity Press.

Birren, J., Woods, A. M. \& Willians, M. V. (1980). Behavioral slowing with age: Causes, organization and consequences. In L. W. Poon (Ed.), Aging in the 1980s, (pp. 293-308), Washington, DC: American Pychological Association.

Brand. N., \& Jolles, J. (1985). Learning and retrieval of words presented auditorily and visually. The Joumal of General Psychology, 1/2(2), 201-210.

Brittain, J. L., La Marche, J. A.w, Reeder, K. P."Roth, D. L., \& Boll, T. J. (1991). Effects of age and IQ on Paced Auditory Serial Addition Task, (PASAT) performance, Cinical Nearopsychologist, 5(2), 163-175.

Campbell, J. I. D. \& Charness, N. (1990). Age-related declines in working menory skills: Evidence from a complex callculation task. Developmental Psychology, 26,879-888.

Cerella, J. (1990). Aging and information-processing rate. In J. E. Birren \& K. W. Schaie (Eds.), Handbook of the Psychology of Aging, (3rd ed., pp. 201-221). New York: Academic Press.

Cerella J. (1991). Age effects may be global, not local: Comment on Fisk and Rogers (1991). Journat of Experimental Psychology: General, 120(2), 215-223. 


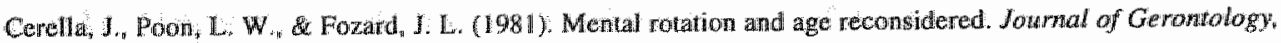
$36(5), 620-624$

Crosistey, M. \& Hiscock, M. (1992). Age-related differences in concurrent-task performance of normal adulis: Evidence for a decline in processing respurces. Psychology and Aging. 7(4), 499.506.

De Vries, H, Vian Houte, L. R., Lindeboom, J, Van Eijk, I. T., \& De Haan, M. (1992). Optellen onder tijdsdruk. Een neuropsychologische test voor thet verdetten van aandacht. (Paced addition: A neuropsychological test of the expacity for divided attention). Tijdschiff woor Gerantologie en Geriatrie, 23(4), 147,156

Deary, I. J., Eomeser, K. P., MacLeod, K. M, Dougall, N., Hepburn, D. A., Frier, B. M., Goodwin, G. M. (1994). PASAT performance and the pattern of uptake of $99 \mathrm{mTc}$-exametazime in brain estimated with single photon emiszion tomography. Biological Psychology, 38(1), 1-18.

Deelman, B. G., Brouwer, W. H. Van Zomeren, A. H. \& Saan, R. J. (1980). Functiestoornissen na trauma capitis (Functional disturbances after thead trauma), In A. Jennekens-Schinkel, J. J. Diamant, H. F. A. Diesfeldt, \& R. Hatuma (Eds.), Nerropsychologie in Nederlond (Neuropsychology in The Netherlands). Deventer, The Ketherlands: Van Loghum Slaterus.

Dufaney, C. L. \& Rogers, W. A. (1994), Mechanisms underlying reduction in Stroop interference with practice for young and old adults. Joumal of Experimental Psychology: Learning, Memory, and Cognition, 20(2), 470-484.

Duncan, J., Burgess, P., \& Emslie, H. (1995). Fluid intelligence after frontal lobe lesion. Neuropsychologia, $33(3), 261-268$.

Dyche, G. M., \& Johnson, D. A. (1991). Development and evaluation of CHIPASAT, an attention test for children: II. Test-retest reliability and practice effect for a normal sample. Perceptual and Motor Skills, 72(2), $563-572$.

Fisk, A. D., Cooper, B. P., Hertzog, C., \& Anderson Garlach, M. (1995). Age-related retention of skilled memory search: Examination of associative learning, interference, and task-specific skills. Journals of Geronfology: Psychological Sciences and Social Seiences, 50(3), 150-161.

Fisk, J. E., \& Warr, P. (1996). Aging and working memory: The role of perceptual speed, the central executive, and the phonological loop. Psychology and Aging, 11(2), 316-323.

Geary, D. C., \& Willey, J. G. (1991). Cognitive addition: Strategy choice and speed-of-processing differences in young and elderly adults. Psychology and Aging, 6(3), 474-483.

Greenwood, P., \& Parasuraman, R. (1991). Effects of aging on the speed and attentional cost of cognitive operations. Developmental Neuropsychology, 7(4), 421-434.

Gronwall, D., \& Wrightson, P. (1981). Memory and information processing after minor head injury. Jonknal of Neurology, Netrosurgery, and Psychiary, 44(10), 889-895.

Gronwall, D. M. (1977). Paced auditory serial-addition task: A measure of recovery from concussion. Perceptual and Motor Skills, 44(2), 367-373.

Gronwall, D. M. A. \& Sampson, H. (1974). The Psychological Effects of Concussion. (Vol. 2). Auckland, Naw Zealand: Auckland University Press.

Fiflartley, J. T. (1986). Reader and text variables as determinants of discourse memory in adulthood. Psychology and Aging, $/(2), 150-158$

Hasher, L.. Stoltafus, E. R. Zacks, R. T. \& Rypma, B. (1991). Age and inhibition. Journal of Experimental Psychology: Leaning. Memory, and Cognition. 17(1), 163-169.

Hasher, L., \& Zacks, R. T. (1988). Working menory, comprehension, and aging: Review and a new view, In G. H. Bower (Ed), The Psychology of Learning and Motivation, (Vol. 22, pp. 193-225). Orlando: Academic Press.

Hishtroudi, S., Chrosniak, L. D., \& Schwartz, B. L. (1991). Effects of aging on priming and skill learning. Prychology and Aging, 6(4), 605-615.

Houx, P. J. \& Jolles, I. (1993). Age arelated decline of psychonotor speed: Effects of age, brain health, sex, and education. Perceptucl and Motor Skills, 76(1), 195-211.

Houx, P. J., \& Jolles, J. (1994). Vulnerability factors for age-related cognitive decline. In R. L. Isaacson \& K F. Jensen (Eds.), The Vuherable Brain and Environmental Risks, (pp. 25-41). New York: Plenum Press.

Houx, P. J., Vreeling, F. W. \& Jolles, J. (1993). Stroop interference: Aging effects assessed with the Stroop Color-Word test Experimental Aging Research, 19(3), 209-224.

Jennings, J. M., \& Jacoby, L. L. (1993). Automatic versus intentional uses of memory: Aging, attention, and control. Psychology and Aging. \&(2), 283-293. 
Johnson, D. A., Roetlig, J. $K$, d Middeton, J. (1988) Development and evaluation of an attentional best for

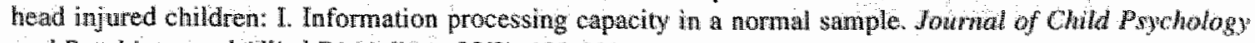
and Psychiatry and Allied Disclphines, 29(2), 199-20\%.

Jolles, J., Houx, P. J., Van Boxtel, M. P. J., Ponds, R. W. H. M. (Eds.) (1995) Manstrich Aging Studty: Determinunts of Cognitive Aging. Mastricht The Nethertands. Neuropsych Publishers.

Mondan, T. C., Rabbit, P. M. (1977), Response times to stimuli of increasting complexity as a function of ageing. British Joumal of Psychology, 68(2), 189-201.

Kane, M. J., Hasher, L. H., Stolefus, E. R., Zacks, R. T. \& Connelly, S. L. (1994) Inhibitory attentional mechanisms in aging. Psychology and Aging, $9(1), 103-112$.

Klein, M. Ponds, R. W. H. M. Houx, P. J. Jolles, J. (1997). Elfect of test duration on age related differences in Stropp Interference. Jourral of Clinical and Experimenwal Newopsychology, 18.11, 77-82.

Korteling. J. E. (1991). Effects of skill integration and perceptual contuptition on age-retated differences in dualtask performance. Fiuman Factors, $33(1), 35-44$

Laver, G., \& Burke, D. (1993). Why do semantic priming effects increase in old age? A meta-anallysis. Psychology and Aging, 8(1), 34-43.

Leininger, B. E., Gramling. S. E., Frarrell, A. D., Kreutzer, J. S., \& Peck, E. (1990). Neuropsychological deficits in symptomatic minot head injury patients after concussion and mild concussion. Jownal of Newrology, Newrosurgery, and Psychiany, 53(4), 293-296.

Lezak, M. D. (1995). Neuropsychological assessment. (3rd ed.). New York: Oxford Uniwersity Press.

Light, L. L., Zelinski, E. M., \& Moore, M. (1982). Adwit age differences in reasoning from new information. Jounal of Experimental Pschology: Learning, Memory and Cognizion, 8, 335.347.

Lorsbach, T. C., \& Simpson, G. B. (1988). Dual-task performance as a function of aduit age and hask complexity. Psychology and Aging, $3(2), 210-212$.

Luce, R. D. (1986). Response times. Their role in inferring elementary mental organization. New York: Oxford University Press.

Luteijn, F., \& Van den Ploeg, F. A. E. (1983). Handleiding Groninger Intelligentertest (GuT) (Manual Groningen buthence Test). Lisse. The Nethentands: Swets \& Zeitlinger..

MacLeod, C. M. (1991). Half a century of research on the Stroop effect: An integratiwe rewiew. Prychological Bulletin, $109(2), 163-203$.

Madden, D. J. (1985). Adult age differences in memory driven selective attention. Developmental Psychology, $2 /(4), 655-665$.

Madder, D. J., Cormelly, S. L., \& Pierce, T. W. (1994). Adult age differences in shifting focused attention. Psychology and Aging. $9(4), 528-538$.

MeDowd, J. M. (1986). The effects of age and extended practice on divided attention performance. Journal af Gerontology, 4l(6), 764-769.

McDowd, J. M., \& Craik. F. I. M. (1988). Effects of aging and task difficulty on divided attention performance. Jownal of Luperinental Psychology: Human Perception and Perfonnance $14(2), 267-280$.

Metsemakers, J. F. M., Höppener, P.. Knotnerus, J. A., Kocken, R. J. J., \& Limonard, C. B. O. (1992). Computerized heallh information in the Netherlands: A registration retwork of family practices. Britsh Journal of General Practice, 42(356), 102-106.

Norman, D. A, \& Shallice, T. (1986). Attention to action: Willed and automatic control of behtivior. In R. J. Davidson, G. E Schwartz, \& D. Shapiro (Eds.). Consciousmess and Self Kegulation, (pp. 1-18). New York: Plentum Press.

Park, D. C., Smith, A. D., Dudley, W. N., Lafromza, V. N. (1989). Erfects of age and a divided attention task presented during encoding and retrieval on memory. Joumal of Experimenal Psychology Learning. Memory. and Cognition, 15(6), 1185-1191.

Plude, D. J., \& Hoyer, W. J. (1986). Age and the sellectivity of visual information-processing. Psychology and Aging, $1(1), 4=10$.

Ponsford, J., Kinsella, G. (1992). Attentional deficits following closed head injury. Journat of Clinical and Experimental Nenropsychology, 14(5), 822-838.

Rabbitt, P. (1991). Mild hearing loss can cause apparent memory failures which increase with age and reduce with $1 \mathrm{Q}$. Acta Otolaryngologica Stockholm, 476(Suppl.) 167-176.

Rarchle, M. E., Fiez, J. A., Videen, T. O., Macleod, A. M. K., Pardo, J. V., Fox, P. T., \& Petersen, S. E. (1994). Practice-related changes in human brain functional anatomy during nonmotor leaming Cerebral Cortex, 4(1), 8-26.

Rey, A. (1964). Lexamen psychologique dans les cas d'encéphalopathie traumatique (Psychological assessment in cases of rraumatic brain injury. Paris: Presses Universitaires de France. 


\section{CHAPTEM 6}

Rogers, W. A. Dertus, E. L., \& Ombert, D. K. (1994). Duallask assessment of age differaces in automatic process development. Psychology and Aging, 9(3), 398-413.

Regers, W. A. \& Fisk, A. D. (1900). A reconsideration of agc-related reaction time slowing from a learning perspective: Age-ralatud stowng is not just complexty-based. Leanung and Individual Differences. 2, 161 179.

Rogers, W. A., \& Fisk, A. D. (1991) Are age differences in consistentmapping visual search due to feature learning or attention trating? Psychology and Aghg, $6(4), 542-550$.

Roman, D. D., Edwal, G, E., Buchanam, R. I, \& Paton, J, H, (1991). Extended noms for the paced auditory serial adotion task. Clinical Newropsychologist, 5(1), 33-40.

Rusf. M. C., Panek, P. E. R Russell, J. E. (1987). Cawtousness and visual selective attention performance among older adults. Jownal of Genetic Psychology, $148(2), 225,235$.

Rush, M. C. Panek, P. E, \& Russel, J. E. (1990). Analysis of individual variability among older adutits on the Stroop Color Word Interferemce Test. International foumal of Aging and Human Development. $30(3)$. $225-236$

Salthouse, T. A. (1985). Speed ol behavio atid its implications for cognition. In J. E. Birren \& K. W. Schate (Eds.), Handboak of the Psychology of Aging, (2nd ed, pp. 400.426). New York: Van Nostrand Reinhold.

Salthouse, T. A. (1988a). Initiating the formalization of theortes of cognitive aging. Psychology and Aging. 3 . $3-16$.

Salthouse, T: A. (1988b). Resource-reduction in interpretations of cognitive aging. Developwental Rewew, 8 . 238-272.

Salthouse, T. A. (1992), Influence of processing speed on adult age differences in working memory, Acta Psychologica, $79(2), 155 \times 170$.

Salthouse, T. A. (1993a). Attentional blocks are not responsible for age-related slowing foumal of Gerontology, $48(6), 263-70$ :

Salthouse, T. A. (1993b), Intluence of working memory on adult differences in matrix reasoning. British Joumal of Prychology, 84(2), $171-199$.

Salthouse, T. A. (1994). The aging of working memory. Special Section: Working memory. Neuropsychology, $8(4), 535,54,3$

Salthouse; 'T. A. \& Babcock, $\mathbb{R}$. L. (1991). Decomposing adalt age differences in working memory. Developmental Psychology, 27, 763-776.

Salthouse, T. A. Fristoe, N. M., Lineweawer, T. T., \& Coon, V. E. (1995). Aging of attention: Does the ability to divide decline? Memory and Cogmition, 23(1), 59-71.

Salthouse, T. A., Rogan, J. D., \& Prill, K. A. (1984). Division of attention: Age differences on a visually presented memory task. Menory and Cognition, 12(6),613-620.

Shallice, T, (1988), From Neuropsychology to Mental Struchere. New York: Cambridge University Press.

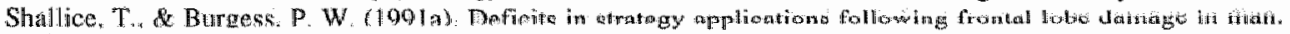
Broin, $1 / 4(2), 727-741$.

Shallice, T., \& Burgoss. P. W. (19916). Higher-order cognitive impaiments and frontal lobe lesions in marn. In 14. S. Levin, H. M. Eisenberg. A. L. Benton (Eds), Frontal Lobe Function and Injury, (pp. 125-138). Now York: Oxford Universily Press.

Shiffin. R. M. \& Schneider, W. (1977). Controlled and automatic human information processing. II: Percepual learniag, automatic attending and a general theory. Psychological Revtew, 84(2), 127\%190.

Sinith, G. A. \& Brower, N. (1995). Slowness and age: Speed-accuracy mechanisms. Psychology and Aging. $10(2), 238: 247$

Spreen, O. B Strauss, E. (1991). A Compendim of Nentopsychological Tesis: Adminnstration. Norms, and Commentay. (Vol XV). New York: Oxford University Press.

Stankov, L. (1986). Age-melated changes in auditory abilities and in a competing task. Mulwariate Behowioral Reseorch, 2/(1), 65-76.

Steywers, J. J. \& Caillard, A.W. (1993). The effects of sleep deprivation and incentives on human performance. Pychological Research, $55(1), 64 m .70$.

Strayer, D. L. \& Kramer. A. F. (1994). Aging and skill acquisition:" Leaming-performance distinctions. Psychology and Aging 9(4), 589-605.

Stuss, D. T. Stethem, L. L., Hugenholte, H., \& Richard, M. T. (1989). Traumatic brain injury: A comparison of three climical tests, and andysis of recovery. Cimical Neuropsychologist, 3(2), 145-156.

Stuss, D. T., Stethem, L. L., \& Poirier, C. A. (1987). Comparison of three tests of attention and rapid information processing across six age groups. Clinical Neuropsychologist, 1(2), 139-152. 
Swabb. D. F. (1991). Brain aging and Allheimer's disease: "Wear and tear" wersus "use it or lose it". Newrobiology of Aging, 12(4), 317-324.

Swaab, D. F., Hofman, M. A. Lucassen, P. J, Salehi, A., Uylings, H. B. (1994). Neuronal atrophy, not cell death, is the main hallmark of Alzheimer's disease. Neumbiology of Aging. 15(3), 369-371.

Taub, H. A. (1973). Memory span, practice, and aging. Joumal of Gerontology, 28(3), 335-338.

Tum, P. A., Wingfield, A., \& Stine, E. A. L. (1991). Speech-processing capacity in young and older adults: A dual-task study. Psychology and Aging, 6(1), 3-9.

Tun, P. A., Wingfield, A., Stine, E. L., \&e Mecsas, C. (1992). Rapid speech processing and divided attention: Processing rate versus processing resources as an explanation of age effects. Psychology and Aging. $7(4)$, $546-550$.

Van Rooij, J. C. G. M., \& Plomp, R. (1991). Auditive and cognitive factors in speech perception by elderly listeners. Acta Otolaryngologica Stockholm, 476(Suppl.), $177-181$. 


$$
\therefore
$$




\title{
Chapter 7
}

\section{The Impact of Aging on Sustained Attention: Time-on-task Effects in Visual Search*}

\begin{abstract}
This study examines the effects of time-on-task on age differences in visual search and the extent to which age effects might be attributed to age-related differences in simple response time, intelligence, sex, and visual acuity. A large cross-sectional study was performed, involving 442 subjects in four age groups (young, 25-35 years; young middle-aged, 40-50 years; old middle-aged, 55-65 years; old, $70-80$ years). Overall age effects were found on response speed and number of correct and false detections. Time-on-task effects on response speed were absent in the old growp. The ability to detect targets or ignore distracting information, however, was susceptible to time-on-task effects, and was thus expressed only at the end of the test. This time-on-task effect suggests that there is an interaction between inhibitory processes and the availability of central processing resources in the oldest group. Simple response time, intelligence, and visual acuity significantly contributed to performance. Statistical analysis showed that changes in visual acuity with age might be an important factor influencing performance in studies of the visual domain. Results are interpreted within the Hasher-Zacks (1988) attentional framework.
\end{abstract}




\section{1}

\section{Introduction}

The predominant view of aging theories holds that the cognitive decline seen with increasing age is the result of a progressive and generalized deterioration in information processing capicity (Cerella, 1990; Salhouse, 1985; 1992). Many studies sought to find an explanation for this phenomenon, all of which attribute age differences in information processing capacity to cognitive mechanism(s) of global effect (Johnson \& Rybash, 1993). In these studies, some athors emphasized the role of perceptuonotor components, whereas others stressed the rolle of computational processes in explaining the extent to which speeded mental processing might be alfected by age (for overviews see: Bashore, 1993; Myerson \& Halle, 1993).

Visual search typically depends on the interaction of perceptuomotor and cognitive processes, two processes that play a changing role in tasks of varying complexity. Visual search also requires endogenous and exogenous initiated shifts of attention, of which the latter can be characterized as being more automatic and reflex than the former (Jonides, Naveh Benjamin \& Palmer, 1985). When a person shifts his or her attention from an old to a new location in a field of inspection, as is the case in visual search, the last attended location is inhibited (Posner \& Dehaene, 1994). Several studies have presented evidence that a decline in this inhibitory capacity is a possible source of attentional decline in the elderly (Hasher, Stoltzus, Zacks \& Rypma, 1991; Kane, Hasher, Stoltzfus, Zacks \& Connelly, 1994; McDowd \& Oseas Kreger, 1991; Tipper, 1991) as distracting information interferes with performance more in older subjects than in younger subjects, and older subjects provide less evidence of inhibitory mechanisms than younger subjects. Kramer, Humphrey, Larish, Logan, and Strayer (1994) however, found equivalent negative priming effects in young adults and elderly people. If subjects have to perform a search task for a prolonged period of time, the ability to inhibit distractor information might be affected in older subjects due to a decline in the availability of processing resources (Crossley \& Hiscock, 1992; McDowd \& Craik, 1988; Plude \& Hoyer, 1986; Salthouse, 1988; Tun, Wingfield, Stine \& Mecsas, 1992). In visual search this might be expressed quantitatively by a slowing of the response time, or qualitatively by fewer correct detections, or by more false detections. Studies by Posner and Dehaene (1994), and by Posner, Snyder, and Davidson (1980) suggest that orienting to a spatial location also involves ficititatory mechanisms that serve to enhance the processing of target items. However, there is little direct evidence for such a mechanism.

The objective of this study was to examine how test duration affects the ability of subjects of different ages to suppress non-relevant information in a visual search task. As inhibitory mechanisms decline with age, we expected time-on-task effects in older subjects to be expressed as an increase in the number of false detections. Because inhibitory and -possiblyexcitatory mechanisms decline with age, we expected older subjects to make fewer correct detections. If the visual search task takes a long time to complete, the limited availability of central processing resources in elderly people might mean that fewer performance-enhancing inhibitory and facilitatory mechanisms are available. We therefore hypothesized that the attentional performance of these subjects would be poorer at the end of the visual search task than at the beginning. The relative contribution of the initial differences in simple response time associated with age, intelligence, sex, and binocular visual acuity were measured after correction fot initial differences in speed with age in a regression analysis. This was done because Tomporowski and Simpson (1990) found intelligence-related differences in sustained attention to be related to the avalability of attentional resources. Finally, in contrast to the 
majority of studies performed up till now, we did not compare only "young" and "old" subjects; but also included "young middle-aged" and "old middle-aged" subjects in onder to detect at which age changes are manifest.

\section{$7.2 \quad$ Method}

\subsubsection{Subjects and Procedure}

This study was conducted as part of a large cross-sectional tesearch program into biologioal and psychological determinants of successful and pathological cognitive aging, named the Maastricht Aging Study (MAAS; Jolles, Houx, van Boxtel \& Ponds, 1995). Subjects were recruited from a register of patients of general practices in the region of Maastricht (Metsemakers, Höppener, Knottnerus, Kocken \& Limonard, 1992). This register contains all relevant past and current medical morbidity as documented by the general practitioners. Subjects with previous or current medical conditions with known impact on cognitive and/or motor functions were excluded from the selection. Exclusion criteria were overt cerebrovasculat disease, chronic neurological pathology (e.g., dementia, epilepsy, parkinsonism), mental retardation, chronic cardiovascular or pulmonary disease, and psychotropic drug use. All subjects were thus normal and healthy according to accepted gerontological criteria. The subjects who were eligible for the study completed a postal questionnaire and participated in an extensive neuropsychological investigation. The standardized postal questionnaire yielded a subjective report on medical history and demographic information, such as age, sex, and education. Educational levell was measured by using a Dutch scoring system (De Bie, 1987) which consists of an 8-point scale, ranging from unfinished primary education (level 1) to university education (level 8 ). Subject sampling was stratified by 12 discontinuous age classes ( 25 years $\pm 1,30$ years $\pm 1,35$ years $\pm 1, \ldots, 80$ years \pm 1 ) and sex. For this study subjects were assigned to one of four age categories, namely between 25 and 35 years (group 1), between 40 and 50 years (group 2), between 55 and 65 years (group 3), and between 70 and 80 years (group 4$)$.

Table 7.1

Demographic characteristics of normal, healthy subjects $(N=442)$

\begin{tabular}{|c|c|c|c|c|c|c|c|c|c|c|c|}
\hline \multirow[b]{2}{*}{ Subjeuts } & \multicolumn{2}{|r|}{$N$} & \multicolumn{2}{|c|}{ Age } & \multicolumn{2}{|c|}{ Education } & \multicolumn{2}{|c|}{ Intelligence } & \multicolumn{2}{|c|}{ Visus } & \multirow{2}{*}{$\frac{\operatorname{sen} \%}{(M / H)}$} \\
\hline & $n$ & $(\%)$ & $M$ & $S D$ & $M$ & $S D$ & $M$ & $S D$ & $M$ & $S D$ & \\
\hline Total group & 442 & $(100)$ & 510 & 16.5 & 3.7 & 2.2 & 112,3 & 11.7 & 1.14 & 0.39 & $50 / 50$ \\
\hline Young & 121 & $(274)$ & 30.6 & 4.2 & 4.7 & 1.9 & $\| 12.3$ & 10.4 & 1.32 & 0.37 & $50 / 50$ \\
\hline Young middle-aged & 120 & $(27.1)$ & 45.6 & 4.3 & 3.9 & 20 & 111.9 & 12.4 & 1.27 & 0.37 & $48 / 52$ \\
\hline Old middleaged & 115 & $(26.0)$ & 60.6 & 4.3 & 3.2 & 2.2 & 112.4 & 13.4 & $\stackrel{\|, 06}{n, 06}$ & 0.30 & $50 / 49$ \\
\hline Odd & 86 & $(19.5)$ & 74.5 & 3.9 & 2.5 & 2.0 & $1 \llbracket 2.7$ & 9.8 & 0.79 & 0.29 & $51 / 49$ \\
\hline
\end{tabular}

Note. Visus: bimoneular visual acuity determined by Landolt-C optolype chart.

Four hundred sixty-six subjects were included and 10 subjects were excluded because of invalid or unreliable results. Since a higher $\mathbb{Q} Q$ might be a confounding variable, the four age 
groups were matched with respect to intelligence. This was done by excluding subjects from group 4 who had an IQ score above 127. This meant that 14 of the initial 100 subjects in this group had to be excluded. This was done because although a one-way analysis of variance did not show there to be significant differences between the $\mathbb{L Q}$ scores of the groups $(F(3,455)=$ $2.40, n .8$.$) . Duncan"s multiple range test showed that group 4$ had a higher $1 Q$ at the .05 level. The characterstics of the remaining 442 subjects whose data were used for analysis are summarized in Table 7.1. In order to correct for outliers or extremes, we excluded subjects with a response time or number of false detections of more than 2.5 standard deviations above the mean or a number of correct detections which was more than 2.5 standard deviations below the mean on either block. Because of temporal cohort effects, the edwcational level declined significantly from 25 to 80 years. This decline was also reflected in the distribution of the mean educational level among the different groups in our study $(F(3,440)=22.51, p<.001)$. All groups were significanty different at the 0.05 level. Visual acuity also declined with age ( $F(3$, $438)=49.23, p<.001$ ); groups 1 and 2 had a similar binocular visual acuity.

7.2 .2

Measures

Intelligence Test. Four subtasks of the Groningen Intelligence Test (GIT; Luteijn \& van der Ploeg, 1982) were administered to obtain a reliable estimation of IQ. The GIT is a test of general intelligence.

Visual Acuity. Binocular visual acuity was determined using the Landolt-C optotype chart, at a distance of 5 meters, under standard luminescence and with corrected vision (Hollwich, 1989).

Simple Response time Task. In this task, the subject is requested to hold down, with the index finger, a red home button. When a target button lights up, the subject has to press this button with the same finger. The home button has to be held down immediately after the target button is pressed until the next button lights up. The measure used for analysis is the motor initiation response time.

Visual Search Task. In the visual search task, stimuli are presented in a pseudo-random order on a computer video monitor ( $640 \times 480$ pixels resolution). Twenty stimuli are randomly assigned to a $10 \times 6 \mathrm{grid}$, all stimuli being 2.5 centimeters apart. Stimuli are small squares of 3 $x 3$ millimeters; targets are defined as four stimuli forming a square of $2.5 \times 2.5$ centimeters. Every 1000 milliseconds two stimuli are moved to a different location in the matrix. Subjects, who sit 60 centimeters from the monitor, have to respond to targets with their dominant hand by pressing a button as quickly as possible. At the center axis the visual angle horizontally is $11.8^{\circ}$; the visual angle vertically is $7.1^{\prime \prime}$. Thus subjects have to move their eyes to be able to detect all targets. Subjects have to respond to targets within 3000 milliseconds, otherwise the targets disappen. The total number of stimuli presented is 750 . With 45 targets, this gives an overall signal probability of 0.06 . The task lasts 12.5 minutes. Targets are never consecutive stimuli. Three targets are presented every 50,000 milliseconds and the same number of stimuli are presented in each quadrant of the inspection area. Before the actual task is performed, there is a practice session consisting of 15 blocks in which subjects obtain feedback on how well they are doing on the task. 
The mean response time for 15 consecutive targets was calculated: There were three clusters with 15 targets and 235 non-targets each. The first cluster of targets is referred to as block 1 . and the third cluster of targets as block 3. Performance was assessed by the number of correct detections (CD, positive responses to targets) and the number of false detections (FD, positive responses to non-targets). In order to measure time-on-task effects, performance in block 1 was contrasted to performance in block 3 .

Overall time-on-task effects associated with aging were analyzed by using repeated measures analysis of variance with age (four levels) and time (two levels) entered as variables. Since visual acuity corrected for age correlated with the mean response time $(-12, p<.01)$ and with the mean number of correct detections $(.20, p<.001)$, we used visual acuity as a covariate in our analysis. The level of significance was set at $p<.05$, to indicate which test variables were liable for subsequent post-hoc analysis. Post-hoc analyses of time-on-task effects within age groups and age effects on performance on blocks 1 and 3 were performed by analysis of covariance also corrected for visual acuity $(p<.05)$.

In order to decide which intra-individual factors might contribute to performance, we entered the initiation time of the Simple Response time Task, intelligence, sex, visual acuity, and age in a stepwise hierarchical multiple regression model. Dependent variables were mean response time on the visual search task, CD, and FD for blocks 1 and 3 together.

\section{$7.3 \quad$ Results}

\section{Response time}

Repeated measures analysis of variance showed significant effects of age and time (Figure 7.1; $F(3,424)=26.37, p<.001$ and $F(1,424)=21.39, p<.001$, respectively). An Age $x$ Time interaction, however, could not be demonstrated $(F(3,424)=1.50$, n.s. $)$. The expected effects of age on response time were found on both blocks 1 and $3(F(3,424)=12.48, p<.001$ and $F(3,424)=22.45, p<.001$, respectively). Analysis of covariance for time-on-task effects clearly showed that groups 1,2 , and 3 became faster during the task $(F(1,119)=9.69, p<$ $.01 ; F(1,116)=7.51, p<.01$, and $F(1,111)=12.18, p<.01$, respectively). The oldest group, however, did not benefit from practice $(F(1,78)<1)$. The younger age groups were faster than their successive older groups at the .01 level. On block 1, however, the response times of groups 2 and 3 were not statistically different, nor were the response times of groups 2 and 3 in block 3 .

\section{Correct Detections}

As hypothesized, we found that the mean number of correct detections (Figure 7.2) was influenced by age $(F(3,420)=8.99, p<.001)$ and time $(F(1,420)=20.47, p<.001)$ to roughly the same extent. Test duration and age also interacted $(F(3,420)=4.49, p<.01)$ and this could be attributed to the fact that the oldest group made significantly fewer correct detections in block $3(F(1,74)=23.49, p<.001)$, whereas the performance of the other groups stayed at the same level $(F(\mathbb{1}, 119)=<1 ; F(1,119)=1.59$, n.s., and $F(\mathbb{1}, \| 10)=$ 2.84, n.s., respectively). 
Thgure 7.1 (left): Mexn puertormance of subjects aged 25-80 years on viseal search response lime vertical bars denoive SEMS.

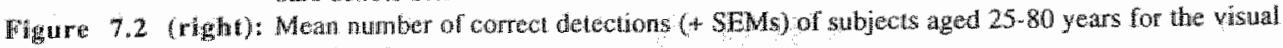

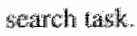
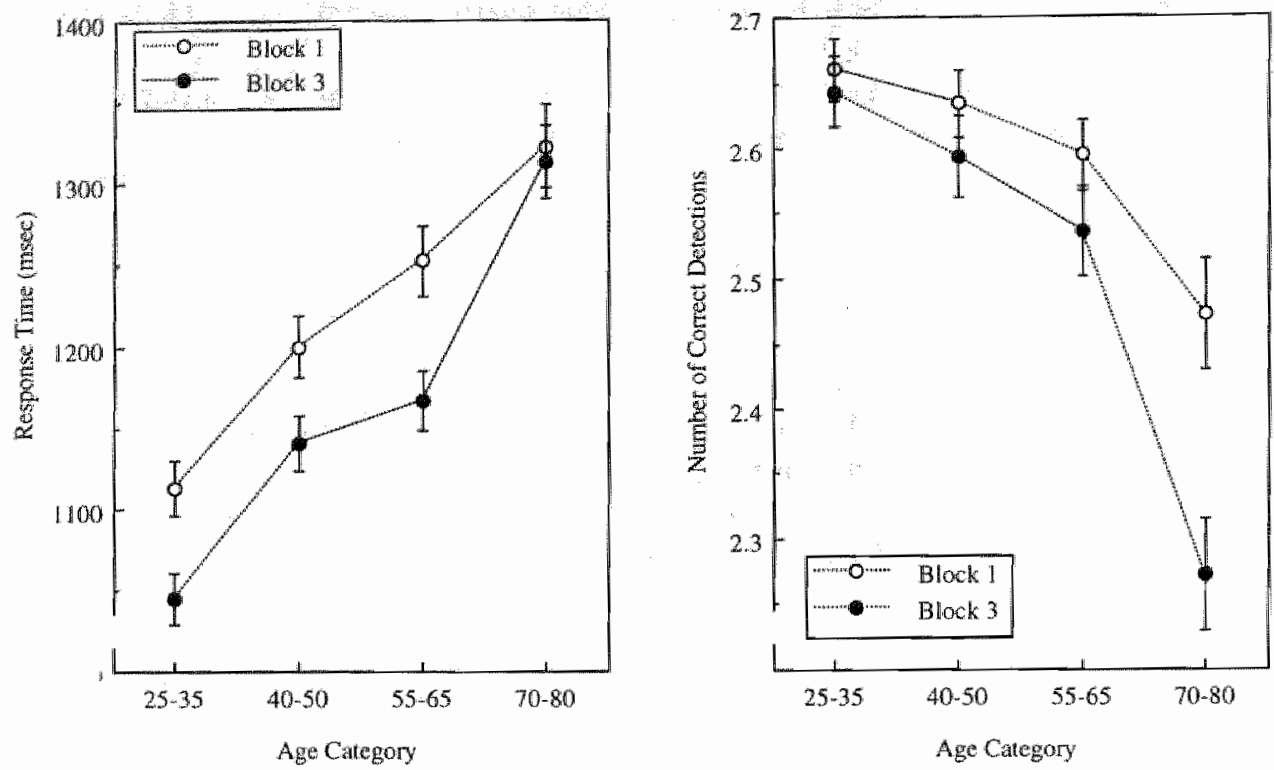

The effect of age on the ability to detect targets was found to be susceptible to time-on-task effects, and was thus expressed only in block 3 (Block 1: $F(3,421)=2.34$, n.s., and Block 3 : $F(3,421)=10.82, p<.001)$. The decline in performance in block $3 \mathrm{can}$ be explained by the fact that the oldest group had significantly fewer correct detections than the other three groups $(F(1,198)=30.02, p<.001 ; F(1,197)=18.69, p<.001$, and $F(1,192)=13.35, p<$ .001 , respectively). Groups 1,2 , and 3 did not differ at the .05 level. When wisual acuity was used as covariate, the apparent effects of age on performance on block 1 were eliminated (noncovaried: $F(3,421)=7.03, p<.001)$.

\section{False Detections}

Repeated measures analysis demonstrated that increasing age tended to be associated with an increase in the number of false detections, as can be seen in Figure $7.3(F(3,429)=4.63, p<$ .01). Time seemed to have a positive effect, since all subjects improved their ability to suppress distractor information $(F(1,429)=51.52, p<.001)$. No Age $x$ Time interaction could be demonstrated $(F(3,429)<1)$. As was the case with the number of correct detections, the ability to ignore distracting information was also susceptible to time-on-task effects, as indicated by a significant decrease in the number of false detections made in block 3 compared to block 1 (block 1: $F(3,430)=2.47$, n.s., and block 2: $F(3,430)=3.55, p<.05)$. Performance in all groups improved over time (groups 1, 2, 3, and $4: F(1,119)=13.88, p<.001 ; F(1,1.19)=$ 
$16.12, p<.001 ; F(1,110)=12.46, p<.01$, and $F(1,82)=9.63, p<.01$, respectively). The age effect found in block 3 can be atcributed to the fact that the oldest group made signilicantly nore false detections than the youngest group $(F(1,205)=14.37, p<.001)$. The perfomance of the other groups did not differ at the .05 level. When visual acuity was introduced as covariate, the apparent effects of age on performance in block I were eliminared (non-covaried: $F(3,430)=3.99, p<.01)$.

\section{Regression Analysis}

The results of the stepwise hierarchical multiple regression model are summarized in Table 7.2. Simple speed, intelligence, visual acuity, and age significantly contributed to the total explained variance $(28 \%)$ in visual search response time. Sex, however, did not make a significant contribution. The same predictors could explain $19 \%$ of the total variance in the number of correct detections. Again, sex did not explain a significant percentage of the total variance. With regard to the number of false detections, visual acuity and age had a significant contribution of a mere $4 \%$.

Figure 7.3: Mean number of false detections (+ SEMs) of subjects aged $25-80$ years for the wisual search lask.

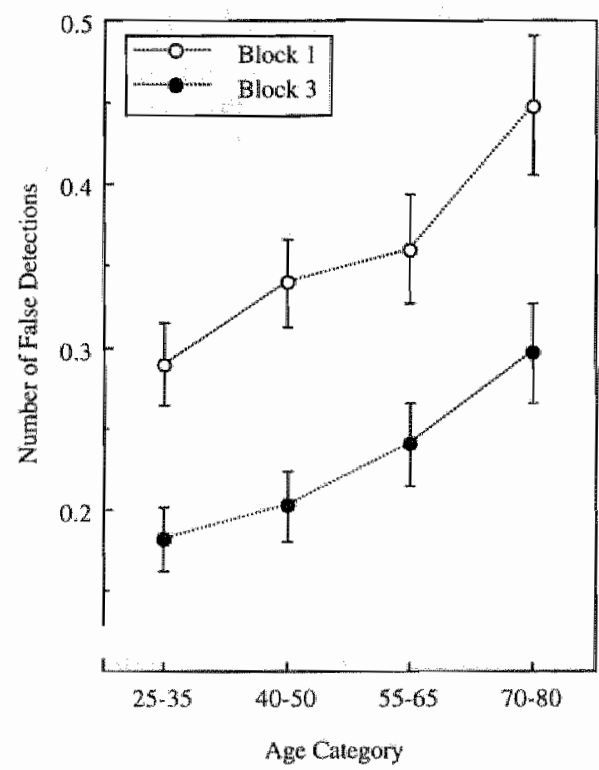


The 7.2

Summany of Stepwise Wiecarehicall Multiple Regresion Analysis for Variables Predicting Visual Gearch Performance $(N=442)$

Predictors Beta $\quad 10 R 2 \quad F \quad$ of

Response time

$\begin{array}{lrrrrr}\text { Simple RT } & .306 & .094 & 43.37 & (1,419) & .000 \\ \text { Frultiligence : } & -.131 & .017 & 7.91 & (2,418) & .005 \\ \text { Ses } & .062 & .004 & 1.73 & (3,417) & .190 \\ \text { Visus } & -.185 & .030 & 14.45 & (4,416) & .000 \\ \text { Age } & .453 & .136 & 78.48 & (5,415) & .000\end{array}$

Contet Detections

$\begin{array}{lrrrrr}\text { Simple RT } & .309 & .096 & 44.25 & (1,419) & .000 \\ \text { Intulligence } & .180 & .032 & 15.07 & (2,418) & .000 \\ \text { Sex } & .038 & .001 & 0.66 & (3,417) & .415 \\ \text { Visus } & .199 & .035 & 17.41 & (4,416) & .000 \\ \text { Age } & .184 & .023 & 11.69 & (5,415) & .000\end{array}$

False Detections

$\begin{array}{lrrrrr}\text { Simple RT } & .006 & .000 & .01 & (1,428) & .909 \\ \text { Inteligence } & -.030 & .001 & .37 & (2,427) & .542 \\ \text { Sex } & -.004 & .000 & .01 & (3,426) & .930 \\ \text { Visus } & -.118 & .012 & 5.17 & (4.425) & .023 \\ \text { Age } & .208 & .029 & 12.68 & (5,424) & .000\end{array}$

Note. Visus: binocular visual acuity determined by Landolt-C optotype chart.

\subsection{Discussion}

The objective of this study was to examine how the ability to suppress non-relevant intormation in a visual search task might be affected by test duration with increasing age. Results in the first place showed the widely documented effects of age on response time, with younger subjects. performing faster than older subjects (Madden, 1986; Madden \& Allen, 1995; Maylor \& Rabbitt, 1994; Plude, Hoyer \& Lazar, 1982). With respect to this general phenomenon, Cerella (1991) concluded that aging is accompanied by a generalized slowing of the central nervous system and that this slowing uniformly affects all stages of information processing. What is particularly striking in our findings is that the oldest group did not benefit from practice (i.e. the response time did not decrease during the task), suggesting that the hypothesized general slowing might not be linear in nature. Because of the large sample used in the present study, and the fact that we incorporated a "young middle-aged" and an "old middle-aged" group, the present data show that this lack of a practice effect is confined to subjects older than 70 years. There might be several explanations for the lack of practice effects in these subjects. According to Madden and Allen (1995), age-related changes in visual search performance involve a decline 
in the efficiency of sampling the amount of evidence necessary to exceed a clecision criterion. In our study, this might have meant that the older subjects continuously needed nore time to decide whether a target was present or not, thus leading to longer response times and a lack of practice effects.

Because there is a decline in inhibitory and -possibly-excitatory mechanisms with age, we expected older subjects to make fewer correct detections. Spatial attention precuing experiments showed that orienting to a spatial location indeed involves both facilitory and inlibitory components (Posner et al., 1980). These components appear to be subserved by different brain areas that can be selectively amplified or suppressed as a function of attentional set (Posner \& Dehaene, 1994). Inhibitory processes play a role in the processing of non-target items, whereas excitatory processes serve to enhance target items. Hasher and Zacks (1988) proposed that inhibitory abilities decline with age, and this proposal is supported by many studies (Gerard, Zacks, Hasher \& Radvansky, 1991; Hartman \& Hasher, 1991; Hasher et al., 1991; Kane et al., 1994; McDowd \& Filion, 1992; McDowd \& Oseas Kreger, 1991; Stoltzfus, Hasher, Zacks, Ulivi \& Goldstein, 1993; Tipper, 1991), all of which basically conclude that older adults seem to process distractors but at the same time fail. to engage inhibitory mechanisms in their rejection of distracting stimuli. Hasher \& Zacks (1988) proposed that there is an age-related breakdown in inhibitory mechanisms that control the content of working memory. Dempster (1992) stated that resistance to interference (i.e., the capacity for inhibition) is a major factor in the development of intellectual competence and declining mental ability later in life. The notion of a general decline in inhibitory mechanisms with increasing age, analogous to a general decline in information processing capacity (Salthouse, 1988), was rejected some years later by Kramer et al. (1994), who concluded that a decrease in the efficiency of inhibitory processing with increasing age is not a general phenomenon, and is not applicable to negative priming effects, response compatibility effects, spatial precuing effects, and self-reported cognitive failures.

Direct evidence for a decline in excitatory mechanisms with increasing age is virtually nonexistent. An indication that this might actually be the case can be indirectly deduced from Positron Emission Tomography (PET) studies in subjects suffering from dementia. When a subject is instructed to pay attention to one particular aspect of the stimuli, blood flow is significantly increased in area V5, the cortical temporo-parieto-occipital junction (Howard et al., 1995; Zeki et al., 1991). This area may also mediate motion vision processing, which is a prerequisite in most visual search tasks (Hotson, Braun, Herzberg \& Boman, 1994). Petersen (Petersen, Corbetta, Miezin \& Shulman, 1994) assumed that the superior parietal cortical region mediates shifts in spatial attention or visual gaze to peripheral locations. In subjects suffering from dementia, PET showed hypometabolism predominaritly in area V5 and in the middle temporal cortical regions (Salmon \& Franck, 1989a, 1989b). The decrease in metabolic rate and the severity of dementia were positively correlated. Other PET studies also showed bilateral hypometabolism in area V5 (Sharp et al., 1986), or in the frontal association cortex in addition to a reduction in the rate of glucose metabolism in area V5 (Pappata, Chabriat, Levasseur, Legault Demare, \& Baron, 1994). However, these dara are not necessarily typical for normal cognitive aging. The studies by Salmon and Franck (1989a, 1989b), suggest that there is a continuum, ranging from normal cognitive aging, through minor cognitive impairment to pathological aging, with each step being accompanied by a gradual decrease in metabolic rate.

We found a decline in the number of correct detections with age. This might be interpreted as being caused by a decline in both facilitatory and inhibitatory mechanisms, although psychological studies will have to be undertaken to prove this point. The increase in the number 
of false detections with increasing age certainly points to there being a deficit in inhibitory processes, since the older subjects seemed to be less able to ignore irrelevant information, as shown when a subject decided to respond when he or she should not have. The fact that the time-on-task effects only became apparent in block 3 points at yet another mechanism to explain the deficits in vistal search performance of the older subjects. The capacity aspect of attention, in the sense of the limited processing resources that underlie task performance, appears to decrease with age (Crossley \& Hiscock, 1992; McDowd \& Craik, 1988; Plude \& Hoyer, 1986; Salthouse, 1988; Tun et al., 1992). A possible lack of energy to sustain the processes of arousal and perceptual processing, as a result of time-on-task, could not be compensated by the mobilization of extra effort in the oldest group. Steyvers and Gaillard (1993) suggested that the influence of time-on-task is not due to a lack of arousal, but is due to a reallocation of resources, which might be more successfull in younger subjects. Steyvers furthermore noticed that when subjects are not rewarded for their performance, they seem to start the task by doing their utmost, and thus spend resources early at the beginning of the task. In a study on the effect of time-on-task on Stroop interference in young and old subjects, Klein, Ponds, Houx, and Jolles (1997) found that the first test part could efficiently differentiate between young and old subjects. A 20-second finger tapping test also showed differential time-on-task effects between young and old subjects (Houx, Vreeling \& Jolles, 1993). The limited availability of central processing resources in the elderly as a consequence of time-on-task might give rise to a decrease in the availability of performance-enhancing inhibitory and facilitatory mechanisms.

A more straightforward explanation for the age differences is that older subjects might make fewer eye movements than the number of objects to be counted and that they might overlook objects at the edges of the visual field (Noro, 1983). Since the time given to detect a target in the visual field ( 3000 milliseconds) was much longer than the minimal time neccessary for all age groups, this explanation is highly unlikely.

In conclusion, the results of our study suggest that there is an interaction between the sustained allocation of resources and inhibitory mechanisms. The limited availability of resources in older subjects might be affected by time-on-task effects and results in a decline in performance-enhancing inhibitory and-maybe-facilitatory mechanisms, thus leading to a decline in performance. These resources are used to support information processing when visual processing becomes difficult because of an age-related deterioration of the visual system. Because these resources are reallocated, they are unavailable to more central cognitive processes, such as the storage and retrieval functions of working memory, so that "upstream" processing of visual information is adversely affected. Inhibition may provide a marker for the early detection of syndromes characterized by a breakdown in cognitive processes. The apparent age-related cognitive breakdown in the visual domain might, however, be reduced after correction for visual acuity.

\section{$7.5 \quad$ References}

Bashore, T. R. (1993). Differential effects of aging on the neurocognitive functions subserving speeded mental processing. In J. Cerella, J. Rybash, W. Hoyer, \& M. L. Commons (Eds.), Adult Infonnation Processing: Limits on Loss. (pp. 37-76). San Diego, CA: Academic Press.

Cerella J. (1990). Aging and information processing rate. In J. Birren \& K. W. Schaie (Eds.), Hondbook of the Psychology of Aging, (3rd ed., pp. 201-221). San Diego, CA: Academic Press. 
Cerella, J. (1991). Age effects may be global, not local comment on Fisk and Rogers (1091). Jommal of Experimenal Pychalog: (oneral, $120(2), 215223$.

Crossley, M. Hiscock, M. (1992). Age-rekted differences in concurtant-task performance of nomal actuls: evidence for a decline in processing resources. Psyohology and A gug, $7(4), 499,506$.

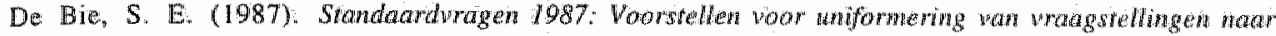

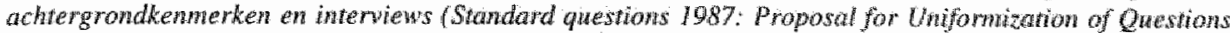
Regarding Backgrowa Variables and hterwews). (2nd ed.). Leiden. The Netherlands. Leiden University Press.

Dempster, F. N (1992). The rise and fall of the inthibitory inechanism: Towand anthed theory of cognitive devellopment and aging. Developmental Review, $12(1), 45-75$.

Gerard, L, Zacks, R. T., Hasher, L., Raduanshy, C, A. (1991). Age deticits in retrioval the fan eftect. Jounnal of Gerowtology, 46(4), $131-136$

Harman, M., \& Hasher, L. (1991). Aging and suppression; memory for previowsly relevan intormation. Psychology and Aging, 6(4), 587-594.

Hasher, L., Stolufus, E. R.; Zacks, R. T., Rypma, B. (1991). Age and inhibition. Jownal of Experimental Psychology: Learnimg Menory, and Cogmition, $17(1), 163-169$.

Hasher, L., \& Zacks, R. T. (1988). Working menory, comprehension, and aging: review and a new wiew. In $\mathrm{G}$ H. Bower (Ed), The psychology of learning and motivation, (Vol. 22, pp. 193225). Orlando: Academic Press.

Hollwich. F. (1989). Leerboek ooghedkunde (Textbook of Ophalmalogy). Unecht: Bohin, Scheltema \& Holkema.

Hotson, J., Braun, D., Herzberg. W., \& Bonan, D. (1994). Transcranial magnetic stimulation of sxtrastrate cortex degrades human motion direction discrimination. Viston Research, 34(16), 2115-2123.

Houx P. A Vreeling, F. W., \& Jolles, J. (1993). Age-related decline of sustained tapping perlormance: Calender or Health? In G. E. Stelmach \& V. Homberg (Eds.), Sensoriworor Impatiment in the Eidenty, (pp. 31-39). Dordrecht, The Netherlands: Kluwer Academic Publishers.

Howard, R. J., Bullmote, E, Brammer, M., Willams, S. C. R. Mellers, J., Woodruff, P., David, A., Andrew, C., Allin, M., Simmons, A., \& Cox, T. (1995). Activation of area VS by visual perception of motion demonstrated with echoplanar MR imaging. Magnetic Resonance Imaging, 13(6), 907-909.

Johnson, S. H. Rybash, J. M. (1993). A cognitive neuroscience perspective on age-telated slowing developmental changes in the functional architecture. In J. Cerella, J. Rybash, W. Hoyen, \& M. L. Commons (Eds.). Adult Infomation Processing: Limits on Loss, (pp. 143-173). San Diego, CA: Academic Press.

Jolles, J., Houx, P. I., van Boxtel, M. P. J., \& Ponds, R. W. H. M. (1995). Macstricht Aging Study. Detenninants of Cogmitive Aging. Mastricht, The Netherlands Neuropsych Publishers.

Jonides, I., Naveh Benjamin, M., \& Primer, J. (1985). Assessing automaticity. Special Issuc: Action, attention and antomaticity. Acta Psychologica, $60(2-3), 157-171$.

Kane, M. J., Hasher, L., Staltzlis, E, R., Zacks, R. T., \& Connelly, S. L. (1994). Inhibitory attentional mechanisms and aging. Pyychology and Aging. $9(1), 103-112$.

Klein, M., Ponds, R. W. H. M., Howx, P. J., \& Jollks, J. (1997). Effect of test duration on agewelatod differences in Stroop Interference. Joumal of Chnical and Experinemal Neuropsychology, 18(1), 77-82.

Kramer, A. F., Humphrey, D. G. Larish, J. F wogan, G. D., \& Strayer, D. L. (1994). Aging und inhibioion: beyond a uhitary wiew of inhibitory processing in attention. Psychology and Aging, $9(4), 491.512$.

Luteijn.F., \& Van der Ploeg. F. A. E. (1982). Handkeidirz Groninger Inteligente Test (Marual Gronimgen Intelligence Test) Lisge: Swats \& Zeilinger B. K.

Madden D. J. (1986). Adult age differences in the attentiomal capacity dernands of visual search. Cognitive Developmen. $J(4), 335-363$

Madden, D. J., \& Allen, P. A. (1995). Aging and the speed/accuracy relation in visual search: Evidence for an accumulator model. Optomery and Vision Science, 72(3), 210-216.

Maylor, E. A., \& Rabbitt, P. M. A. (1994). Applying Brinley plots to individuals: Effect of aging on performance distributions in two speeded tasks. Psychology and Aging, 9(2), 224-230.

McDowd, $J_{\text {. M. }}$ \& Craik, F. I. (1988). Effects of aging and task difficulty on divided attention performanca. Joumal of Experinemal Psychology. Hawnd Pexception and Performance, 14(2), 267-280.

McDowd, J. M, \& Filion, D. L. (1992). Aging, selective attention, and inhibitory processes: A. psychophysiological approach. Psychology and'Aging, 7(1),65-71.

McDowd, J. M. \& Oseas Kreger D. M. (1991). Aging, inhibitory processes, and negative priming. Jounal of Gerontology, 46(6), 340-345. 
Metsemaker, I: F. M, Hoppener, P.; Knotumerts, J. A., Kocken, R. J, J.3 Limonard, C. B. G. (1992). Computerized health information in the Netherlands: a registration metwork of family practices. British Jounal of Gentral Practice, $42(356), 102-106$.

Myerson, I., Hale, S. 1993). General wowing and age inwariance in cognitive processing: the other side of the coun. In J. Cerello, I. Rybash, W. Hoyer, \&. L. Commons (Eds), Adult Infonnation Processing: Limits on Loss, (pp. 15-141). Swin Diego, CA Acadernic Press.

Noro, K. (1983) A descripute model of visual search Human factors, 25(1), 93-101.

Pappata, 5. Chabriat, 11. Levasseur, M, Legaull Demare, F, \& Baron, J. C. (1994). Marchuafava-Bignami disease with dementa: severe cerebral metabolic depression revealed by PET. Jow racal of Neural Tronsmission.

Parkinsons Disease and Denenta Section, $8(1-2), 131-137$.

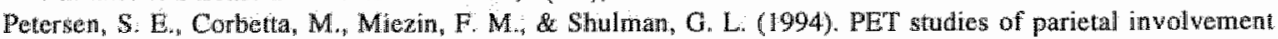
in spatial attention: Comparison of different task nypes. Special Issue Sthifts of visual attention Camadian. Journal of Experimental Psychology, 48(2), $319-338$.

Plude, D. J., Hoyer, W. I. (1986): Age and the selectiwity of visual information processing. Psychology and Aging. $f(1), 4-10$

Plude, D. J., Hoyer, W. J., \& Lazar, J. (1982). Age, response complexity, and target consistency in visual search. Experimental Aging Resechch, 8(2); 99-102.

Posmer, M. I., Dehane, S. (1994). Atientional networks. Trends in Neurosciences, 17(2), 75-79.

Posner, M. I., Snyder, C. R., \& Davidson, B. 3. (1980). Attention and the detection of signals, Joumal of Experinental Psychology: General, 109(2), 160-174.

Salnon, E, Franck, G. (1989a). Positron emission tomographic study in Alzheimer's disease and Pick"s disease. Archives of Gerontology and Geriatrics. Supplement $1,241-247$.

Salmon, E., \& Franck, O. (1989b). Positron emission tomography in degenerative dementias. Acta Neurologica Belgica, $89(3-4), 150-155$.

Salthouse, T. (1992). Mechanisms of age-cognition relations in adulthood. Hillsdale, NJ: Erlbaum.

Salthouse, T. A. (1985). A Theory of Cogritive Aging. Amsterdam: North Holland.

Salhouse, T. A. (1988). Resource-reduction in interpretations of cognitive aging. Developmental Review: 8 , 238-272.

Sharp, P., Gemmell, H., Cherryman, G., Biesson, J., Crawford, J., \& Smith, F. (1986). Application of iodine123-labeled "sopropylamphetamine imaging to the study of dementia. Jownal of Nuclear Medicine, 27(6), $761-768$.

Steyvers, F. J, \& Gaillard, A. W. (1993). The effects of sleep depriwation and incentives on human performance. Psychological Research, 55(1), 64-70.

Stolizfus, E. R., Hasher, L, Zacks, R. T., Ulivi, M. S., Goldstein, D. (1993). Investigations of inhibition and interference in younger and older adults. Joumals of Gerontology, 48(4), 179-188.

Tipper, S. P. (1991). Less attentional selectivity as a restle of dechining inhibition in older adults. Bullefin of the Psychonomic Somery, 29(1), 45-47.

Tomporowski, P. D., \& Simpson, R. G. (1990). Sustaned attention and intelligence. Intelligence, $14(1), 31$ 42.

Tun, P. A., Winglield, A., Stinc. D. A., Mecsas, C. (1992). Rapid speech processing and divided attention: processing rate versus processing resources as an explanation of age effects. Piychology and Aging, $7(4)$, $546-550$.

Zeki, S., Watsom, J. D., Lueck, C. J., Friston, K. J., Kennand, C, E Frackowiak, R. S. (1991). A dircet dentonstration of functional specialization in human visual cortex. Joumal of Neuroscience, $7 /(3), 641-649$. 


\title{
Chapter 8
}

\section{Long-term Persisting Cognitive Sequelae of Traumatic Brain Injury and the Effect of Age*}

\begin{abstract}
This study examined the notion that mild to moderate traumatic brain injury (TBI) may have persistent effects which become evident upon neurocognitive testing in a phase in which the effects of physiological aging become manifest. Neurocognitive pefformance was tested in 25 middle-aged and 20 old subjects who had sustained mild to moderate TBI on average several decades earlier. The TBI subjects regarded themselves as normal and healthy.

The performance of the TBI subjects was inferior to that of matched healthy controls on all aspects of primary and secondary memory and on the majority of tests used to measure speed of performance. There was no interaction between the effects of TBI and those of age, and the performance of middle-aged TBI subjects was similar to that of old controls. The results are taken to indicate that TBI sustained earlier in life may cause permanent sequelae in specific domains of cognitive functioning and that it might attenuate the age-related decline in cognitive functioning. Most strikingly, however, these deficits were not perceived as a limiting factor in everyday life, which suggests that coping strategies may be important.
\end{abstract}

* This chapter has been published as:

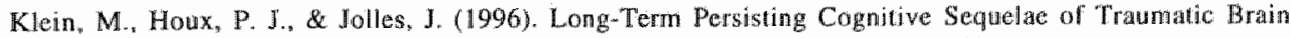
Injury and the Effect of Age. Journat of Nenows and Mental Disease, 184(8), 459-467. 


\subsection{Introduction}

It is well-established that mild to moderate traumatic brain injury (TBI) may give rise to the development of postconcussional symptoms, in which both physiogenic and psychogenic intluences have an mportant role (Bohnen \& Jolles, 1992; Lishman, 1988). The Hollywood head injury myth - which suggests that the hero can sustain a severe blow to the head, but still go on in the next scene without any apparent ll effects-may have contributed to the persisting skepticism about the role of organic factors in the development of a postconcussional syndrome (Evans, 1992): Nowadays, ample evidence derived from neuropathological (Leestma, 1991), neurophysiological (Hayes, Stonnington, Lyeth, Dixon, \& Yamamoto, 1986; Schoenhuber \& Gentilini, 1989), neuroimaging (Levin, Willams, Eisenberg, High, \& Guinto, 1992), postmortem (Oppenheimer, 1968), and neuropsychological studies (Binder, 1986; Bohnen, \& Jolles, 1992; Green, Leon, Dieter, Benfante, \& Walker, 1992) strongly suggests that organic factors might be involved in postconcussional syndromes. These findings prompted the proposal that the postconcussional syndrome should be recognized as an etiological entity to be classified in DSM-IV (Brown, Fann, \& Grant, 1994).

In subjects with mild to moderate TBI, postconcussional symptoms may persist well beyond 6 months (Alves, Macciocchi, \& Barth, 1993), 1 year (Middleboe, Andersen, Birket, \& Friis, 1992; Rutherford, Merrett, \& McDonald, 1979), or even 3 to 5 years (Edna \& Cappelen, 1987), which suggests that the effects of mild to moderate TBI might not be completely reversible. Levin and collegues (1987) however, suggest that a single uncomplicated mild TBI produces no permanent disabling neurobehavioral impairment. Yet several potential risk factors for persisting postconcussional symptoms have been identified, of which lower educational, intellectual, and socioeconomic level, sex, alcohol abuse, repeated TBI, and skull fracture are the most important. Age has also been identified as a possible risk factor for persisting postconcussional symptoms (Edna, \& Cappelen, 1987; Evans, 1992). A rather alarming neuropsychological follow-up study by Mazzucchi et al. (1992) showed diffuse deterioration in $28 \%$, moderate deterioration in $25 \%$, and dementia in $21 \%$ of TBI subjects older than 50 years, which implies that mild TBI could be followed by severe consequences. Similar, though less dramatic, results ware obtained in studies by Houx, Jolles, and Vreeling (1993), and Houx, Vreeling, and Jolles (1991b), who found that biological life events (BLE), such as mild TBI, could accentuate the effect of "normal" biological aging in subjects who considered themselves to be normal and healthy. The ustal age-related decline in cognitive functioning, as found in tasks involving new information, planning of new activities, and effortful, controlled processing (Botwinick, 1981; Craik \& Byrd, 1982), and the general slowing of behavior (Salthouse, 1985) might become manifest in middle-age, when the normal biological aging process becomes evident. From the results of a multiple cohort study suggested that BLE might even give rise to predementing stages of Alzheimer's disease (Jolles, Houx, Vreeling, \& Verhey, 1993). Houx and coworkers, however, did not investigate whether the objectively established cognitive deficits in BLE-aflected subjects could be correlated to subjective ratings of cognitive functioning. The results of a controlled postal survey carried out 1-5 years after mild TBI, Bohnen et al. (1994) concluded that mild TBI may produce long-term neurasthenic symptoms.

The objective of the study was first to test the hypothesis that neurocognitive performance is compromised in patietns with mild-to-moderate TBI many years after the trauma, even in subjects who do not have complaints. This hypothesis was tested by comparing healthy 
middle-aged and elderly subjects with mild to moderate TBI in their history to matched healthy control subjects. The second objective of the study was to evaluate whether there is an interaction between age and TBI. An interaction of age and group in a two-way ANOVA design would be indicative for a cumulative effect of age and TBI, whereas group effects without an interaction with age would indicate that mild to moderate TBI in otherwise healthy subjects attenuates the cognitive deterioration due to age. For this reason, two age groups of TBI subjects were compared. The choice of the neuropsychological tests was based upon earlier studies by Bohnen, Jolles, and Twijnstra (1992), Houx, Vreeling, and Jolles (1991a), and Houx and coworkers (1991b).

\subsection{Method}

\subsubsection{Subjects}

Male and female volunteers who had recovered from TBI and who considered themselves as being normal and healthy were invited to participate in the study by means of an editorial in several local newspapers. Forty-five subjects with mild to moderate, uncomplicated TBI were eventually selected out of the 110 subjects who initially replied. The remaining subjects did not meet the inclusion criteria. Subjects were preselected over the telephone to ensure that none of them had a medical history of complicated TBI (i.e., bone or skull fractures), a major psychiatric illness known to be characterized by cognitive deficits, previous brain damage caused by stroke or disease, alcohol or drug abuse, impaired vision or hearing, or an insufficient command of Dutch. The time elapsed since the trauma was set at minimally 1 year, in order to include only subjects who were in a stable phase. All participants considered themselves as completely recovered. Only subjects who were aged between 40 and 59 years (group I; middle-aged) or older than 60 years (group II; elderly) were included in the study. Two control groups of 45 volunteers were drawn from a larger population of subjects who were normal and healthy ( $N=262$; Houx et al, 1991a) and free from previous TBI. Control subjects were selected by means of matching to the individual TBI subjects with respect to age ( \pm 1 years), sex, and level of education ( \pm 1 point). The latter was assessed by a Dutch scoring system (Verhage, 1964) which comprises a scale ranging from 1 ("primary education not finished") to 7 ("university degree"). There were thus two TBI groups and two control groups with individually matched pairs. The mean age at testing of middle-aged and old TBI subjects was 49.4 and 69.8 years, respectively; the mean age of controls was 48.9 and 70.4 years, respectively. In both the experimental group and the control group there were 10 male and 15 female middle aged, and 10 male and 10 female old subjects. The mean educational level of middle-aged and old controls was 4.1 and 3.5, respectively. In TBI subjects this was 3.9 and 3.1 , respectively. These differences were not statistically significant.

The procedure for recruitment of the controls has been described elsewhere (Houx et al., 199 lb). In short, they were recruited by means of adwertisements placed in local newspapers and were paid for their participation. The advertisement stressed that participants should be healthy. All participants underwent the same procedure of neuropsychological evaluation as the experimental group. All subjects were free of significant somatic, neurological and/or psychiatric disorders, alcohol abuse, TBI, and skull fractures. Depression scores for the controls are not available, but all subjects denied complaints of lowered mood, nor did they 
report significant problems with memory or concentration. The control subjects can thus be regarded as a representatiwe selection of the middle-aged and old population (Houx et al., 1993 , 1991a).

\subsubsection{Procedure}

After informed consent was obtained, the subjects who were eligible for the study answered a postal questionnaire received a neurocognitive evaluation which was part of an extensive neuropsychological inwestigation. The standardized postal questionnaire yielded a subjective report on medical history and cognitive functioning after TBI. Zung"s self-rating scale for depression (Zung, 1965) and the 47-item Utrecht Coping List (Schreurs, Van de Willige, Tellegen, \& Brosschot, 1988) were completed, as were a checklist of postconcussional symptoms, which distinguished between typical postconcussional symptoms and rather aspecific psychovegetative functional symptoms (Bohnen. Twijnstra, \& Jolles, 1992), and a self-rating scale concerning quality of sleep (Mulder-Hajonides van der Meulen, 1980). Severity of TBI as a possible predictor of outcome was estimated by asking the subjects about coma duration, memory loss, and postconcussional complaints. Coma duration and memory loss were scored on a 4 -point scale as less than 15 minutes, 15 minutes to 1 hour 1 to 24 hours, or more than 24 hours. The duration of postconcussional complaints was also scored on a 4 point scale as being either less than 1 week, 1 to 4 weeks, 1 to 6 months, or 6 months and longer. The subsequent summation of the scores on the three scales yielded a crude measure of the severity of 'TBI. This estimation of severity is consistent with that of the alternative to the Glasgow Coma Scale suggested by Stambrook, who in his study with mild to severe TBI patients used post-traumatic amnesia, coma duration, and age as indirect markers of TBI severity (Stambrook, Moore, Lubusko, Peters, \& Blumenschein, 1993).

\subsubsection{Neurocognitive Tests}

The Visual Verbal Learning Test (VVLT). This test is a version of the Rey Auditory Verbal Leaming Test (Brand \& Jolles, 1985; Lezak, 1995). The test consists of a list of 15 monosyllabic and concrete nouns in Dutch, which are presented in five trials on a computer screen. The nouns occur very frequently and are acquired early in life. Items are presented in the same sequence at a rate of one per 2 seconds. Each trial ends with a free recall of the words (free immediate recall). Twenty minutes after the fifth trial, the subject is requested to recall as many words as possible (free delayed recall). A yes/no recognition test, consisting of the 15 former words and 15 new but sinilar words, is given after the delayed recall test. The variables used are: number of correctly recalled words on the first trial as a measure of primary memory; the total number of correct words over the five trials as a measure of learning capacity; the number of correct words on delayed free recall as a measure of retrieval from secondary memory; and the number of correct responses on delayed recognition as a measure of memory consolidation.

The Stroop Color Word Test (SCWT; Lezak, 1995) is a perceptual interference task and consists of three cards. The test examines the speed at which color names are read (SCWT card I) and the speed at which colored patches are named (SCWT card II). The SCWT card III involves color names again, but the printing ink is different from the color name. The variables 
are: response times for cards I, II, and III. Interference is expressed by the percentage of extra time needed for card III, relative to the average of the first two cards:

$$
\frac{\text { SCWTII }-5}{5(\text { SCWTI }+ \text { SCWTI })} * 100 \%
$$

The Concept Shifting Test (CST: Houx \& Jolles, 1994) is an adaptation of the Trail Making Test, which is a test of visual conceptual and visuomotor tracking. The CST consists of three subtasks. In each subtask, the subject has to mark small circles with digits or letters in ascending order on an A4 sheet of paper. The subject is requested to do this as quickly as possible. On each test sheet, 16 small circles are grouped in a larger circle. In the smaller circles, the test items (numbers (CSTA), letters (CSTB), or both (CSTC)) appear in a fixed random order. The subjects are requested to cross out the itens in the right order, in the same way as in the TMT. In three identical "zero-tasks" all 16 empty circles are to be crossed out clockwise as quickly as possible. The variables are: response time in CSTA and CSTB as a measure of wisual conceptual and visuomotor tracking, and CSTC as an index of concept shifting ability. The relative contribution of the need to shift between two concepts to the total time needed for subtask $\mathrm{C}$ (percentage) is conveniently computed with the formula:

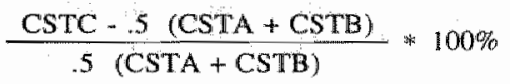

where CSTA, CSTB, and CSTC denote the total time taken to complete the test part. The averaged time for the three zero-tasks is used as a measure of basic motor speed.

Intelligence. Intelligence was estimated by using the Groningen Intelligentie Test (GIT; Luteijn \& Van der Ploeg, 1983), which is a test of general intelligence.

\subsubsection{Rating Scales}

Zung"s self-rating scale for depression (Zung, 1965). This self-rating scale for depression was used to measure depression as a clinical entity. The crude tolal score is expressed as a percentage of the maximum score (80), yielding an index of depression.

The Utrecht Coping List (Schreurs et a1., 1988). This questionnaire contains 47 items describing seven styles of coping. Factor analytically derived coping styles are: active problem solving, seeking distraction, avoidance and passive expectancy, seeking social support. depressive reaction, expression of emotion and anger and comforting cognitions.

Postconcussional symproms (Bohnen et at., 1992). This factor analytically derived checklist of postconcussional symptoms consists of a factor concerning typical postconcussional symptoms (11 high-loading items: PCS-I), and of a factor concerning rather aspecific psychovegetative functional symptoms (13 high-loading items: PCS-II).

Sleep quality (Mulder-Hajonides van der Meulen, 1980). Subjective sleep quality was rated on a 14-item, 5-point scale questionnaire covering aspects of sleep, such as the initiation and maintenance of sleep, sleep interruptions, return to sleep after waking early in the morning, capability to function at peak efficiency upon waking, and the total time the subject reports sleeping at night. The crudle total score is expressed as a percentage of the maximum score (70), yielding an index of subjective sleep quality (good sleep quality $=100 \%$ ). 


\section{$8.2 .5 \quad$ Statistical Analysis}

In order to estimate global age, TBI, or interaction effects, values of VVLT trials 1 through 5 , Stroop subtasks I, II, and II, and CST subtasks $A, B$, and $C$, were used in a repeated measures ANOVA. The accepted level of significance was set at 05 to indicate tests worth using for further analysis. In subsequent andyses with regard to memory performance, a two-way ANOVA was performed by using the following variables: number of correctly recalled words on the first trial the total number of correct words over the five trials; the number of correct words on delayed free recall and the number of correct responses on delayed recognition. For analysis of attentional performance, the time needed for Stroop subtasks I, II, and II and the interference score were used in a two-way ANOVA to analyze differences between TBI and controls. The relative contribution of the need to shift between two concepts lio the total time needed for subtask $\mathrm{C}$ and the average time needed for the three ' 0 -tasks' were processed in the same way as in the test for memory and attentional performance. The accepted level of significance for all two-way ANOVAs was set at 05. A two-way ANOVA was used to estimate the accuracy with which the various tasks were performed, as reflected by the number of errors, corrections, omissions and double responses. This analysis did not reveal any significant differences between groups on the neuropsychological tests.

Zung's self-rating scale for depression, the 47-item Utrecht Coping List, the checklist of postconcussional symptoms, and the rating scale for quality of sleep were compared to test performance and injury-related variables by using Pearson"s $\mathrm{s}$.

\section{3}

\section{Results}

Table 8.1

Characteristics of middle-aged and old subjects who sustained a nilld to moderate TBI

\begin{tabular}{|c|c|c|c|c|c|c|}
\hline & \multicolumn{2}{|c|}{$\begin{array}{l}\text { Middle-aged } \\
(n=25)\end{array}$} & \multicolumn{2}{|c|}{$\begin{array}{l}\text { Old } \\
(n=20)\end{array}$} & \multirow[b]{2}{*}{$t$} & \multirow[b]{2}{*}{$p$} \\
\hline & $M$ & Range & $M$ & Range & & \\
\hline Intetligence (GIT) & 113.6 & $(87.0-132.0)$ & 115.2 & $(87.0-133.0)$ & -0.46 & nis. \\
\hline $\begin{array}{l}\text { Zung s self-rating scale for depression } \\
\text { Postconcussional Symptomst }\end{array}$ & 49.3 & $(27.5 \cdot 713)$ & 46.0 & $(32.5-66.3)$ & 1.10 & n.s. \\
\hline PCS-IS Symptoms & 24.6 & $(16.0-34.0)$ & 21.9 & $(14.0-32.0)$ & 1.81 & n.s. \\
\hline PCS II Symptoms & 218 & $(14.0-33.0)$ & 20.9 & $(15.0-33.0)$ & 0.62 & m.s. \\
\hline Sleep Questionnaire & 61.3 & $(28.0-86,0)$ & 61.4 & $(42.0-83.0)$ & -0.02 & $n . s$. \\
\hline Severity of "TBI" & 3.8 & $(2.0 \cdot 7.0)$ & 3.7 & $(2.0-8.0)$ & 0.24 & n.s. \\
\hline Time elapsed since TBI (yrs) & 26.4 & $(1.0-520)$ & 35.7 & $(2.0-63.0)$ & -1.74 & n.s. \\
\hline W of TBI & 1.5 & $(1.0-4.0)$ & 1.2 & $(1.0-2.0)$ & 0.99 & R.s. \\
\hline Age at TRI & 23.0 & $(5,0-49,0)$ & 34.1 & $(2.0-78.0)$ & -2.02 & $<.05$ \\
\hline
\end{tabular}

Note. a Tho crude rotal soore is expressed as a percentage of the maximum score $(80)$.

b. Sce texi for explanation.

- Parcentage of maximal possible complaints concerning sleep.

\section{Patient Characteristics}

Evaluation of the claaracteristics of the patients in the two TBI groups (Table 8.1) showed that there were no statistically significant differences between the two groups except for age at the 
time of TBI: the middle-aged subjects were younger than the old subjects at the time the TBI was sustained $(t=-2.02, d f=43, p<.05$ ). There were no differences in severity, number of years elapsed since TBI, or number of TBT, between middle-aged and old TBI subjects $(r=$ $0.24, d f=43$, n.s.; $t=-1.74, d f=43$, n.s. and $t=0.99, d f=43$, n.s., respectively). All subjects reported having sustained cona and memory loss, but three middlemaged and seven old subjects reportedly had no postconcussional complaints. The duration of coma, memory loss, and postconcussional complaints was not correlated (Pearson's $r$ ) with neuropsychological outcome, but the age at which TBI was sustained was positively correlated with visual conceptual and visuomotor tracking as measured by CST (CSTA: $r=32, p<.05$ and CSTB: $r$ $=.41, p<.01$ ). Psychometric differences between the main test wariables of TBI subjects and controls are summarized in Table 8.2 .

Table 8.2

Psychometric differences between subjects who sustained a mild to moderate TDI and controls: Performance on main test variables.

\begin{tabular}{|c|c|c|c|c|c|c|c|c|c|c|c|}
\hline \multirow[b]{3}{*}{ Variable } & \multicolumn{4}{|c|}{ Controls $(n=45)$} & \multicolumn{4}{|c|}{$\mathrm{TBI}(n=45)$} & \multirow{3}{*}{$\begin{array}{l}\mathrm{TBI} \\
\text { Eiffect }\end{array}$} & \multirow{3}{*}{$\begin{array}{l}\text { Age } \\
\text { Biflect }\end{array}$} & \multirow{3}{*}{$\begin{array}{c}\text { Interaction } \\
\text { Effect. }\end{array}$} \\
\hline & \multicolumn{2}{|c|}{ Middle-Aged } & \multicolumn{2}{|c|}{ Old } & \multicolumn{2}{|c|}{ Middle Aged } & \multicolumn{2}{|c|}{ Old } & & & \\
\hline & $M$ & $S D$ & $M$ & $S D$ & $M$ & $S D$ & $M$ & $S D$ & & & \\
\hline \multicolumn{12}{|l|}{ 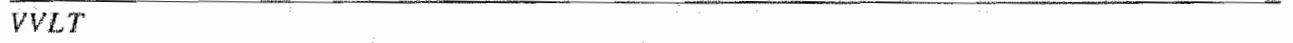 } \\
\hline Triall 1 & 6.2 & 1.4 & 5.7 & 1.4 & 5.2 & 1.6 & 4.5 & 1.7 & $<.001$ & n. $s$. & H.s. \\
\hline Total in 5 trials & 50.8 & 6.2 & 49.0 & 5.8 & 44.2 & 7.5 & 38.6 & 9.8 & $<.001$ & $<.05$ & n.s. \\
\hline Delayed recall & 11.4 & 1.9 & 11.3 & 2.3 & 8.6 & 3.1 & 7.1 & 2,8 & $<.001$ & n.s. & n.s. \\
\hline Dellayed recognition & 14.3 & 1.0 & 14.9 & 0.2 & 14.0 & 16 & 13.8 & 1.6 & $<.05$ & n.s. & n.s. \\
\hline \multicolumn{12}{|l|}{ SCWr } \\
\hline SCWT Card II & 39.0 & 5.5 & 45.3 & 4.3 & 48.0 & 9,0 & 48.7 & 7.5 & $<.001$ & $<.05$ & m.s. \\
\hline SCWT Card II & 52.5 & 7.7 & 61.1 & 8.2 & 62.1 & 13.9 & 62.4 & 9.1 & $<.05$ & $<.05$ & 11. s.s. \\
\hline SCWT Card III & 83.3 & 13.7 & 103.8 & 15.6 & 98.5 & 30.4 & 113.3 & 22.5 & $<.01$ & $<.001$ & n. \\
\hline SCWT \% Slowing & 82.7 & 22.4 & 959 & 28.8 & 77.9 & 26.8 & 103.4 & 24.0 & n.s. & $<.001$ & n. 8 \\
\hline \multicolumn{10}{|l|}{$\operatorname{Csp}$} & \multicolumn{2}{|c|}{$\frac{1}{y}$} \\
\hline CST Subtask A & 23.3 & 8.6 & 25.3 & 9.6 & 22.5 & 6.3 & 27.6 & 7.0 & Hi.s. & $<.05$ & $\mathrm{~m}, \mathrm{~s}$ \\
\hline CST Subtask & 26.3 & 9.6 & 29.0 & 10.8 & 27.1 & 9.0 & 34.2 & 14.2 & $\mathrm{n} . \mathrm{s}$ & $<.05$ & ก.: \\
\hline CSI Subtask C & 37.2 & 17.3 & 43.2 & 23.3 & 40.2 & 39.2 & 43.2 & 14\rfloor$. & n.s. & $<.05$ & n.s. \\
\hline CST W Sllowing & 47.1 & 32.8 & 53.9 & 34.8 & 37.4 & 25.2 & 40.8 & 20.4 & $n s$. & $\mathrm{mis}$. & n.s. \\
\hline Zaro- Task & 5.9 & 1.4 & 6.9 & 2.3 & 6.9 & 2.5 & 8.2 & 3.3 & $<.05$ & $<.05$ & ty.s. \\
\hline
\end{tabular}

Note. Figures for Means and Standard Deviations represent time in secands except for Visual Verbal Leawng Test in which the number of words recalled are presented.

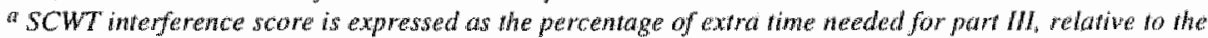

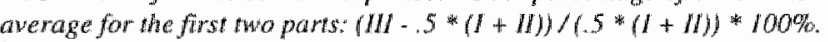

b The relative contribution of the need to shift between two concepis to whe folat tha needed for subark $C$ (percentage) is computed with the formula: (CSTC-.5*(CSTA+CSTB)) $(.5 *(C S T A+C S T B)$ * $100 \%$.

cLevels of significance for $\mathrm{CHI}$, age, and interaction effects, n., not significant.

Effects on Memory Performance (Figure 8.1).

Primary memory, expressed as the number of correctly recalled words in Trial 1 , wass affected in TBI subjects, in the absence of age or interaction effects $(F(1,86)=12.19, p<.001, F(1,86)$ 
-3.27, ns.s, and $F(1,86)<1$, respectively). Measures of secondary memory performance were also affected by TBI: Repeated measures ANOVA on trials 1 through 5 showed TBI subjects to perform worse than their matched controls $(F(1,86)=29.20, p<.001)$. Although less pronounced, a significant effect of age was also demonstrated, in the absence of an interaction effect $(F(1,86)=5.56, p<.05$ and $F(4,344)=1.46$, n.s., respectively). Both TBI and aging seemed to reduce the subjects' capacity to learn new material, as expressed by the 5-Trial sum score (two-way ANOVA: $F(1,86)=29.21, p<.001$ and $F(1,86)=5.56, p$ $<05$ ). As far as delayed recall after 20 minutes was concerned, TBI subjects showed deficits in active tetrieval from memory, without age or interaction effects $(F(1,86)=41.68, p<$ $001, F(1,86)=2.26$, n.s. and $F(1,86)=1.56$, n.s., respectively). TBI subjects also had difficulty in passively retrieving material that had been consolidated into memory, $(F(1,86)=$ $6.88, p<05)$. Again, age or interaction effects could not be found $(F(1,86)<1$. and $F(1$, 86) $=2.95$, ns., respectively).

Figure 8.1 (left): Mean performance on Trials 1 through 5, Delayed Recall (DelRcl) and Recognition (Recog) in the Visual Verbal Learning Test, of middle-aged subjects with a mild to moderate TBI versus optimally heaithy age peers, contrasted to the performance of old subjects affected by TBl versus optimally healthy age peers (vertical bars denote Standard Errors of the Mean).

Figure 8.2 (right): Mear performance on three cards of the Stroop Color Word Test, of middle-aged versus old subjects affected by a mild to moderate TBI, contrasted to the performance of their optimally healthy age peers (vertical bars denote Standard Errors of the Mean).
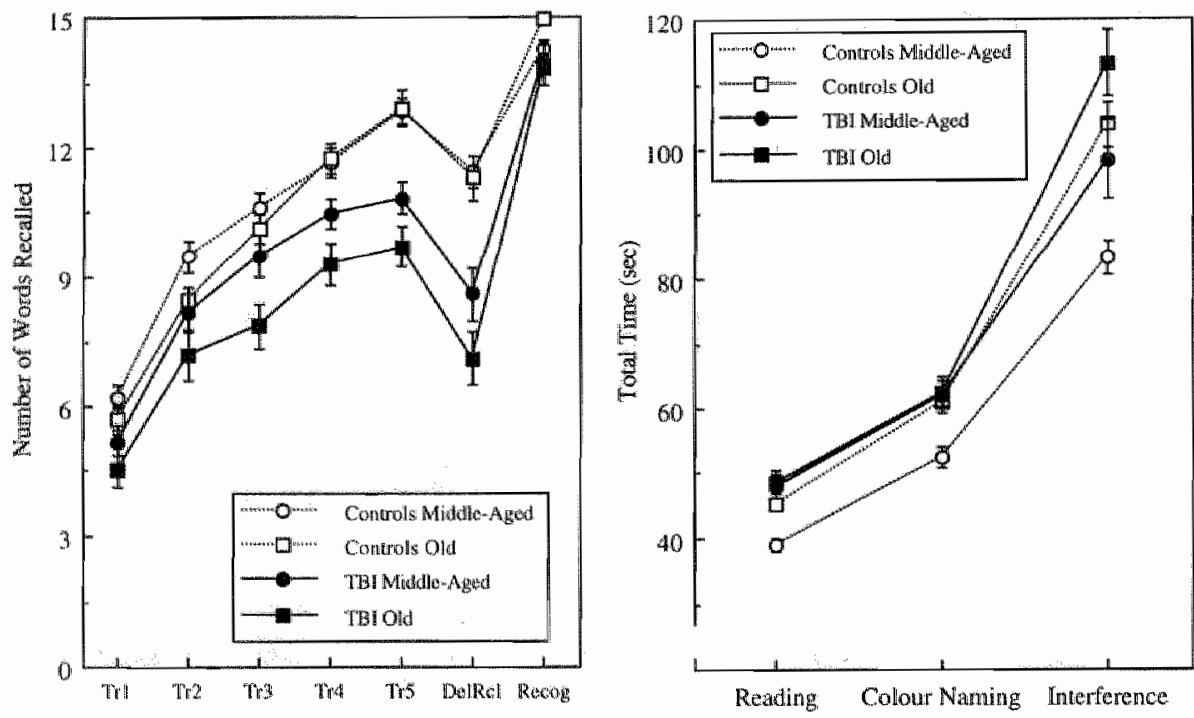

\section{Effects on Stroop and Concept Shifting Tests.}

Repeated measures ANOVA showed that TBI subjects were slower than controls in the Stroop Color Word Test (Figure 8.2), and that old subjects were slower than middle-aged subjects ( $F$ $(1,86)=10.24, p<.01$ and $F(1,86)=11.80, p<.001)$. There was no interaction effect $(F$ 
$(2,170)=1.73$, n.s.). Two-way ANOVA showed sigmificant TBI and age effects on all three cards. TBI tended to have a stronger impact than age on highly antomated skills like reading (card $\mathrm{D},(F(1,86)=17.85, p<.001$, and $F(1,86)=5.78, p<.05$, respectively). TBI and aging had similar effects on color naming (card II) $(F(1,86)=6.29, p<.05$, and $F(1,86)=$ $4.31, p<.05$, respectively). This was not the case for color-word interference (card III), where performance seemed to be mainly affected by age (TBI: $F(1,86)=7.09, p<.01$, and age: $F$ $(1,86)=14.57, p<.001$, respectively). When the amount of interference on card III was

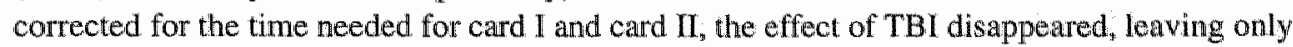
an effect of age (TBI: $F(1,86)<1$, age: $F(1,86)=12.81, p<.001$, respectively), This means that TBI subjects were slower in reading and naming colors but did not show accentuated interference due to TBI.

Overall measures of visual conceptual or visuomotor tracking (CST) revealed effects of age, but not of TBI (repeated measures ANOVA: $F(1,86)=4.86, p<.05$, and TBI: $F(1,86)<1$, respectively, two-way ANOVAs, $F(1,86)=4.36, p<.05 ; F(1,86)=4,59, p<.05$, and $F$ $(1,86)=4.13, p<.05$, respectively). TBI did not affect the performance on the subtasks $A, B$, and $C(F(1,86)<1 ; F(1,86)=1.66$, n.s., and $F(1,86)<1$, respectively). When the performance on subtask $C$ was related to the time needed for subtask $A$ and subtask $B$, the effect of age disappeared (age: $F(1,86)<1$; TBI: $F(1,86)=3.45$, n.s.; interaction: $F(1,86)$ $<1$, respectively). The basic motor speed appeared to be affected by TBI and age to roughly the same extent $(F(1,86)=5.06, p<.05$, and $F(1,86)=5.20, p<.05$; interaction: $F(1,86)<$ 1).

\section{Effects an Psychological Variables}

Zung's self-rating scale for depression was positively correlated only with the scores for SCWT cards I and II $(r=.47, p<.01$ and $r=.41, p<.01$, respectively). According to normative data derived from a nomal, healthy population (Zung \& Durham, 1973), the TBI subjects in our study could not be characterized as suffering from major depression. The TBI subjects who, according to the Zung scores, had a somewhat depressed mood, had a lower perceived quality of sleep $(r=.55, p<.001)$, more PCS-I and PCS-II symptoms, and a more depressive reaction pattern, as measured by the UCL (respectively $r=.74, p<.001 ; r=.72, p<.001$, and $r=.65, p<.001)$. In the TBI subjects intelligence tended to be negatively correlated to self-rating of depression $(r=-36, p<.01)$. Coping styles could not be consistently contrelated to neuropsychological outcome. Active problem solving, as expressed by the UCL, tended to be positively correlated with intelligence $(r=.41, p<.01)$ and negatively with sleep, and PCS-I and PCS-II symptoms $(r=-.39, p<.01, r=-.40, p<.05$ and $r=-.43, p<.01$, respectively). Depressive reaction patterns correlated with complaints concerning sieep $(r=.35$, $p<.05)$, but correlated more strongly with PCS-I and PCS-II symptoms $(r=.62$ and $.59, p<$ $.001)$.

\subsection{Discussion}

One major question of the present study was whether middle-aged or old subjects who are considered to have recovered from mild to moderate TBI could be characterized by a decreased neurocognitive performance compared to that of matched controls. The period between trauma and neuropsychological evaluation in this study extended well beyond the traditional period of 
recovery. A second major question was whether there was evidence that TBI accelerated cognitive aging. The resuls show that the performance of the TBI subjects was significantly inferior to that of the matched controls in all tests of primary and secondary memory used. An effect of age could be demonstrated on the learning capacity, but not on delayed recall. Not only did TBI subjects do less well than controls in consolidating material into memory, but they also showed a marked difficulty in passively retreving this information from memory. These data are new in that, anth now, there were no studies in which neurocognitive performance was evaluated so long after the TBR

The most striking finding of our study is that although the TBB subjects had reportedly made a good recovery and did not have any cognitive complaints at the time of selection for the study, they still performed worse than their matched controls. We found no robust correlation between indices of the severity of TBI and neuropsychological outcome, although the age at which TBI was sustained turned out to be poorly, but positively correlated with visual conceptual and wistomotor tracking scores. This suggests that cognitive sequelae might be relatively independent of the severity of the TBI in our population, which sustained TBI on average three decades earlier. It is possible that, after recovery, the subjects reached a steady state, beyond which their cognitive deficit did not improve. The pattern of the scores on the SCWT of the middle-aged TBI subjects was very similar to the pattern of the old controls (Figure 8.2). The older TBI subjects, however, did not perform disproportionately worse than their younger peers. We consider that these scoring patterns are indicative of accelerated cognitive aging due to the effect of TBI. In the middle-aged TBI subjects, the usual age-related decline in cognitive functioning seemed to occur earlier than in their age-peers not affected by TBI. As such, the data obtained in the present study provide important information with respect to the relation between cognitive aging and age-extrinsic factors, such as mild to moderate TBI. The present results strengthen the notion proposed by Houx et al. (1991a) and Jolles et al. (1993) that minor health-related factors (so-called Biological Life Ewents, BLE) are very important in determining the age at which the physiological aging process leads to cognitive deterioration. It must be emphasized, however, that our population might not be a truly representative sample of the general TBI population, since our subjects represent a group of TBI subjects who voluntarily responded to newspaper advertisements. Moreover, they had to regard themselves as nomal and healthy, and thus subjects with possibly more serious sequelae of previous TBI were excluded. More research is needed in order to deepen our insight into the relation between TBI and age.

As far as the nature of the effects on nevrocognitive performance are concerned, our findings. are similar to those of Levin, Goldstein, High, and Eisenberg (1988), who found that approximately one-fourth of TBI subjects with relatively preserved intellectual functioning manifested defective memory, assessed with both auditory and pictorial measures of memory, when tested 5 to 15 and/or 16 to 42 months after injury. These results should be considered with caution since the patients in Levin's group represented a sample with moderate to severe TBI. Both moderate and severe TBI groups, however, still showed deficits in memory performance 16 to 42 months post-injury. Gupta and Ghai (1.991) found TBI subjects to perform significantly worse on both immediate and delayed free recall. Hall and Bornstein (1991) found that the poorer total recall scores of subjects with mild TBI could not be related to the duration between injury and neuropsychological evaluation and suggested that this poorer performance was a lasting feature of memory function after such an injury. The fact that in the present study coma duration, memory loss, and duration of postconcussional complaints could 
not in any way be correlated with the neuropsychological outcome partly supports the findings of Strugar, Sass, Buchanan, Spencer, and Lowe (1993), who concluded that subjects with mild TBI do not necessarily have to have been unconscious to have memory deficits.

As far as perceptual interference is concerned, Posner and Snyder (1975) concluded from SCWT studies that color naming and reading go on in parallel and without interference until close to the output: "If they result in look-up of the sume name, the overall reaction time is speeded; if they produce different names and a vocal output is required, the word tends to compete with the color name and reaction time is increased". Our TBI subjects needed significantly more time for all cards of the SCWT, as did the older control subjects. With respect to interference (SCWT III), no interaction of age with TBI could be demonstrated. When corrected for the basic speed for cards $I$ and II, only age seemed to be a relevant factor in explaining the extra slowing with card III. This suggests that the TBI subjects in this study did not have a selective attention deficit, but that they did have a more generalized reduced rate of information processing, thus confirming the results of a study by Stuss and collegues (1985). Stuss stated that residual brain damage secondary to TBI might be expressed by an imparred information processing capacity. Reeder and Logue (1.994) stressed the lack of processing resources in explaining memory deficits and even concluded that a possible paucity in attentional capacity can not be held responsible for subsequent memory deficits. The results seem to support the ideas of Symonds (1962), who found it questionable whether even mild TBI would ever be completely reversible.

Although in TBI subjects information processing seemed to be the main cognitive process affected, the literature provides some evidence that there are deficits in perceptual and motor processes (Van Zomeren, Brouwer, \& Deelman, 1984). This would be compatible with the lower basal CST motor speed of TBI subjects in our study. However, visual conceptual and visuomotor tracking, reflected by the CST score, could not distinguish between the two groups. The fact that the TBI subjects did not complain about their cognitive limitations might possibly in part be attributed to the role of coping strategies. Our middlle-aged subjects showed a somewhat more active attitude, i.e., they tended to seek more distraction and social support than the old TBI subjects did. The latter difference proved to be statistically significant. The subjects with alless active coping style tended to have more complaints concerning sleep, as well as more PCS-I and PCS-II symptoms. We might furthermore question whether intelligence also plays a role in determining complaints following TBI According to Sternberg (1982), intelligence enables the subject to acquire, process, classify, and integrate information obtained from the enwironment. This might mean that the more intelligent a person is, the better he or she will be able to deal with cognitive challenges in everyday life. In our study, the more intelligent subjects showed lower self-ratings of depression and a stronger tendency to active problem solving. A lower level of intelligence might in fact accelerate the decline in brain reserve capacity associated with aging and thus might give rise to an earlier onset of clinical symptoms (Satz, 1993). Our TBI subjects had an average intelligence score which was somewhat higher than that of the general population in the Netherlands. This fact thus might have contributed to the observed lack of long-term neurasthenic symptoms in our TBI subjects, although this possibility can only be verified by longitudinal studies.

An explanation for the discrepancy between neuropsychological outcome and cognitive complaints could be that we used self-ratings for coma duration, post-traumatic amnesia, and duration of complaints after TBI rather than the more strict Glasgow Coma Scale. Because posttraumatic amnesia obscures the recall of facts related to injury. TBI subjects usually are not abje 
to give a fairly reliable report, and therefore they compare themselwes with how they were before the rauma. We might, however, question whether in mild to moderate TBI recall is as strongly affected as it is in severe TBI. Another factor that possibly contributes to the discrepancy is the fact that subjective reports of events that took place a long time ago tend to be rather unreliable regardless of the possible effects of post-traumatic amnesia.

In conclusion, this study shows that although subjects who have sustained a TBI many years. prev"ously, are characterized by objective cognitive deficits or even by an attenuated cognitive aging, they do not necessarily have cognitive complaints. This might mean that over the years since they have sustained TBI they have learned to cope with cognitive limitations in everyday life. The fact that our subjects on average were more intelligent might in part have contributed to this: Our findings suggest that future research should aim at determining the extent that personal resources, such as the premorbid level of intelligence, mental stability, and family support can compensate for the decline in brain reserve capacity associated with aging.

\subsection{References}

Alves, W. M., Macciocchi, S. N., \& Barth, I. T. (1993). Postconcussive symptoms after uncomplicated mild head injury. Jownal of Head Trauma Rehabilitations, 8, 48-59.

Binder, L. M. (1986). Persisting symptoms after mild head injury: A review of the postconcussive syndrome. Journal of Cinical and Experimental Neuropsychology, 8(4), 323-346.

Bohnen, N., \& Jolles, J. (1992). Netrobehavioral aspects of postconcussive symptoms after mild head injury. Joumal of Nernous and Mental Disease $180(11), 683-692$.

Bohnen, N., Jolles, J., \& Twijnstra, A. (1992). Neuropsychological deficits in patients with persistent symptoms six months after mild head injury. Neurosurgery, 30(5), 692-696.

Bohnen, N., Twijnstra, A., \& Jolles, I. (1992). Post-traumatic and emotional symptons in different subgroups of patients with inild head injury. Brain Imjury, $6(6), 481-48$ ?

Bohnen, N., Van Zutphen, W., Twijnstra, A., Wijnen, G., Bongers, J., \& Jolles, J. (1994). Late outcome of mild head injury: Results from a controlled postal survey. Brain Injury, 8(8), 701-708.

Botwinick, J. (1981). Neuropsychology of Aging. In S. B. Filskov \& T. J. Boll (Eds. $)_{*}$ Handbook of Clinical Neuropsychology, (pp. 135-171). New York: John Wiley \& Sons.

B tand, N.. Jolles $J_{\text {. }}(1985)$. Learning and tetrieval rate of words presented auditorily and visually. Joumal of General Pychology, 1/2(2), 201-210.

Brown, S. J., Fann, J. R. \& Gtant, I. (1994). Postconcussional disorder: Time to acknowledge a common source of neurobehavioral morbidity. The Jomal of Neuropsychiatry and Clinical Neurosciences, $6(1), 15$ 22.

Craik, F. 1. M., \& Byrd, M. (1982). Aging and Cognitive Deficts: The role of attentional resources. In F. I. M. Craik \& S. Trehub (Eds.), Aging and Cognitive Processes, (pp. 191-211). New York: Plenum Press.

Edna, I. H., Cappelen, J. (1987). Late post-concussional symptoms in traumatic head injury: An analysis of frequency and risk factors. Acta Neurochirurgica (Wier), 86(1-2), 12-7.

Evans, R. W. (1992). The postconcussion syndrome and the sequelae of inild head injury. Neurologic Clinics, $10(4), 815-847$

Green, J. Leon, B. C., Dieter, J., Benfante, P., \& Walker, D. (1992). Somatosensory evoked responses via electronic brain imaging (EBI). Clinical Electroencephalography, 23(2), 79-88.

Gupta, A. \& Ghai, D. (1991). Memory in head injured patients. Joumal of Personality and Clinical Studies, $7(1), 55-61$.

Hall, S. \& Bornstein, R. A. (1991). Serial-position effects in paragraph recall following mild elosed-head injury. Perceptual and Motor Skills, 72(3), 1295-1298.

Hayes, R. L., Stonnington, H. H., Lyeth, B. G., Dixon, C. E., \& Yamamoto, T. (1985). Metabolic and neurophysiologic sequelae of brain injury: A cholinergic hypothesis. Central Nervous System Tramma, 3(2), $163-173$. 
Houx, P. J. \&olles, J. (1994), Wuhnerability factors for age-related coghitive dedine. In R. L. Istakson \& K.

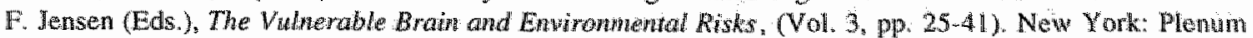
Press.

Houx, P. J., Jolles, J, \& Vreeling, F. W. (1993). Stroop interference: Aging effects assessed with the Stroop Color Word test. Experiwental Agüg Research, 190 3), 209-224.

Houx, P. I., Vreeling, F. W., \& Jolles, J. (1991a). Age-associated cognitiwe decline is related to biologicall life events. In K. lqbal, D. R. C. McLachan, B. Winblad, \& H. M. Wisniewski (Eds), Alzheimer's Disease: Basic Mechanism, Diagnosis and Therapentic Strategies, (pp. 353-358). Chichester, UR: John Wiley \& Sons.

Houx, P. J., Vreeling, F. W. \& Jolles, J. (1991b). Rigorous bealth sereening reduces age efteet on memory scanning task. Brain and Cognition; 15(2), 246-260.

Jolles, J., Houx, P, J., Vreeling, F. W \& Verhey, F. R. J. (1993), Cognitive aging, biological life events and primilive reflexes. Neuroscience Research Communications, 3 (suppl. 1), $\mathrm{S47-550.}$

Levin, H. S., Goldstein, E. C., High, W. M. \& Eisenberg, H, M. (1988). Disproportionately severe memory deficit in relation to normal intellectual functioning after closed head injury. Journal of Nowrology. Newrosurgery, and Psychiatry, 5 l(10), 1294-1301.

Levim, H. S., Mattis, S., Ruff, R. M., Eisenberg, H. M., Marshall, L. F., Tabaddor, K, High, W. M.\& Frankowski, R. F. (1987). Neurobehavioral outcome following minor head injury: A three acenter study. Journal of Neurosurgery; $66(2), 234 \times 243$.

Levin, H. S., Willams, D. H. Eisenberg, H. M., High, W. M. \& Guinto, F. C. (1992), Serial MRI and neurobehavioral findings after mild to moderate closed head injury. Jounal of Nenrology, Neumswrgery, and Psychatry, 55(4), 255-262.

Lezak, M. D. (1995). Neuropsychological Assensmemt. (3nd ed.). New York: Oxford University Press.

Lishman, W. A. (1988). Physiogenesis and psychogenesis in the "post-concussional syndrome". British Jourtal of Psychiatry, $153,460-469$.

Luteijn, F., Van der Ploeg, F. A. E. (1983). Handleiding Groninger Intelligentie Test (GIT) (Manual Groninger Inteligence Test). Lisse, The Netherlands: Swets and Zeithinger.

Mazzucchi, A., Cattelani, R., Missale, G, Gugliotta, M., Brianti, R., Parma, M. (1992). Head-injured subjects aged over 50 years: Correlations between variables of trauma and neuropsychological follow-up. Journal of Neurology, 239(5), 256-260

Middleboe, T., Andersen, H. S, Birket, S. M., \& Friis, M. I. (1992). Minor head injury" Impact on general health after 1 year-A prospective follow-up siudy. Acta Newrologica Scandinavica, 85(1), 5-9.

Mulder-Hajonides van der Meulen, W. R. E. H. (1980), Memsurement of sleep quality. In W. P. Koella (Ed.), Sleep 80: Proceedings of the Fifth European Congress on Steep Research. Basel: Karger.

Oppenheimer, D. (1968). Microscopic lestions in the brain following head injury. Journal of Newrology, Neurosurgen, and Psychiarry, 3/(4), 299-306.

Posner, M. I. \& Snyder, C. R. R. (1975). Attention and cognitive control. In R. L. Solso (Ed.), Information processing and cogmitron, the Loyola Symposinm, (pp. 55-85). Hillsdale, NJ: Lawrence Bitlbaum Associates.

Reeder K. P., \& Logue, P. E. (1994). The effects of tramatic brain injury on inlormation processing. Archives of Climical Neuropsychologi, $9(6), 491$.500.

Rutherford, W. H., Merret, J. D. \& McDonald, J. R. (1979). Symptoms at one year following concussion from minor head injuries. Injury, $10(3), 225-230$

Salthouse, T. A. (1985), Speed of behawiour and its implications for cognition. In J. E. Birren \& K. W. Schaie (Eds.), Handbook of the Psychology of Aging, (2 edt., pp. 400-426). New York: Van Nostrand Reinhold.

Satz, P. (1993). Brain reserve capacity on symptom onset after bran injury: A formulation and rewiew of evidence for threshold theory. Neuropsychology, 7(3), 273-295.

Schoenhuber, $\mathbb{R}$, \& Gentilini, M. (1989). Neurophysiological assessment of mild head injury. In H. S. Levint. H. M. Eisenberg, \& A. L. Benton (Eds.), Mild Head Injwy, (pp. 142-150). Wew York: Oxford University Press.

Schreurs, P. J. G., Van de Willige, G. Tellegen, B., \& Brosschot, J. F. (1988). De Utrechre Copiag Lijst (The Urecht Coping List): UCL. Lisse. Swets and Zeillinger.

Stambrook, M., Moore, A. D., Lubusko, A. A., Peters, L. C. \& Blumemschein, S. (1993). Alternatives to the Glasgow Coma Scale as a quality of life predictor following traumatic brain injury. Archives of Clinical Nenropsychology,8,95-103.

Sternberg, R. J. (1982). Handbook of Haman Intelligence. New York: Cambridge University Press. 


\section{4』 CHAPTER 8}

Strugai, J., Sass; K. J., Euctianan, C. P. Spencer, D. D., Love, D. K. (1993). Long-term consequences of

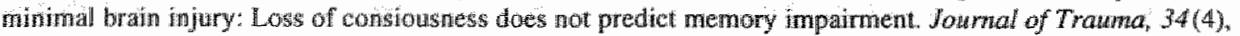
$555-559$.

Stuss, D. T, Ely, P, Hugenbollz, H, Richard, M. T., LuRochelle, S, Poirier, C. A, \& Bell, I. (1985). Subth ncuropsychological deficts in patients with good recovery after closed head injury. Newrosurgery. $17(1)_{1} 41-47$.

Symonds, C. (1962). Concussion and its gequelas Lancet, $1,1-5$.

Van Zomenen, A. H. Brouwer, W. H., Deelman, B. G. (1984). Antentional deficits: The riddles of selectivity, spesd and alerness. In N. Brooks (Ed.). Closed Head Injury. Psychological, Social and Family Consequences: (pp. 74-107), Oxford: Oxfond Universily Press

Verhage, F, (1964). Malligentie en leffid (Intelligence and Age). Unpublished doctoral dissertation. University of Groningen, The Netherthands.

Zung, W. W. K. (1965). A sell-rating depression scale. Archives of General Psychiatry. 9, 334-343.

Zung, W. W. K, Burham, N.C. (1973). From art to science: The diagnosis and treatment of depression. Archines of General Psychatry, 29(3), 328-337. 


\title{
Chapter 9
}

\section{Attentional Performance in Young and Old Patients with Cervical Acceleration / Deceleration (CAD) Injury}

\begin{abstract}
This study examined the influence of cervical acceleration/deceleration injury (CAD) on information processing speed and attentional performance associated with aging. Twenty-nine young ( $<50$ years) and 20 old symptomatic CAD patients $(\geq 50$ years) were compared to 49 healthy controls who were matched with respect to age, sex, and education. CAD patients were mainly characterized by a reduction in information processing capacity under speeded conditions. Performance in a non-speeded self-paced divided attention task, however, was not affected by $\mathrm{CAD}$ injury. This suggests that the limited resources of CAD patients lead to a decline in performance only when time limits are imposed. Overall sustained attention performance did not appear to be affected by CAD injury, but when time-on-task effects were evaluated, $C A D$ patients showed a decrease in vigilance performance. Overall, attentional performance in older CAD patients was not affected more than in younger patients. Self-reported postconcussional symptoms and invested effort might be relatively independent of the attentional deficits after CAD injury. These findings suggest that clinicians should carefully evaluate how $\mathrm{CAD}$ patients reach their performance. A detailed task analysis can thus reveal the cognitive difficulties of CAD patients that lead to limitations in the ability to cope with everyday life situations.
\end{abstract}


Motor wehicle accidents with an acceleration/deceleration mechanism of injury are one of the most common catuses of neck injury (Evans, 1992), with an arinual incidence of about 70 per 100,000 . These figures, however, considerably differ from country to country (Spitzer et al,, 1995). Cervical acceleration/deceleration injury (CAD) is defined as a trauma causing cervical musculoliganent sprain or strain due to acceleration and/or deceleration of the head relative to the trunk in any plane (Hirsch, Hirsch, Hiramoto, \& Weiss, 1988). CAD injury is considered to be a subgroup of mild traumatic brain injury (TBI) and is primarily characterized by the absence of direct head strike in the presence of cervical injury. The absence of physical head trauma does not exclude brain injury, and this is why CAD patients suffer neurologic and/or neuropsychological injuries that are typically seen in TBI patients (Varney \& Varney, 1995). Varney and Varney demonstrated that brain damage without skull impact can result from deceleration from $30 \mathrm{~g}$ to more than $200 \mathrm{~g}$. Ettlin and colleagues (1992) considered their findings in CAD patients to be compatible with possible damage to basal frontal and upper brain stem structures. Ommaya and Hirsch (1971) suggested that angular acceleration of 1800 radians/ $\mathrm{sec}^{2}$ would result in a cerebral concussion in about $50 \%$ of humans. In some frontal impacts at speeds of only $13 \mathrm{mph}$, the acceleration of the head exceeded $1800 \mathrm{radians} / \mathrm{sec}^{2}$.

Apart from neck pain and headaches, that may be referred pain from the upper cervical joints and muscles (Barnsley, Lord, \& Bogduk, 1994), CAD patients have symptoms that constitute the postconcussional syndrome (see chapter 4; Brown, Fann, \& Grant, 1994). Radanov, Dvorak, and Valach (1992) furthermore distinguished two clinically distinct conditions arising from CAD injury. The first syndrome, the "cervicocephalic syndrome", is characterized by headache, fatigue, dizziness, poor concentration, disturbed accommodation, and impaired adaptation to light intensity. The second syndrome, the "lower cervical spine syndrome," is accompanied by cervical and cervicobrachial pain. There is disagreement about whether symptoms persisting beyond 6 months are directly attributable to whiplash injury (Pearce, 1989; Pearce, 1.994). In the absence of identifiable somatic correlates (e.g., neurological or scan findings) complaints continuing after CAD injury tend to be thought of in terms of neurosis or malingering. However, symptoms after $\mathrm{CAD}$ are fairly uniform, and their accurate simulation is inconceivable. In fact, laypeople have a limited knowledge of the range of symptoms that may occur following mild TBI and are particularly unaware of the cognitive deficits (Aubrey, Dobbs, \& Rule, 1989).

The persistence of CAD symptoms can give rise to the "chronic CAD syndrome", which by definition is present when patients remain symptomatic after 6 months (Pearce, 1994). Injury-related cognitive imparment and age are significant factors predicting illness behavior at 6 months post-injury, whereas psychosocial factors, negative affectivity, and personality traits were not (Radanov, Di Stefano. Schnidrig, \& Ballinari, 1991). A history of pretraumatic headache significantly increases the likelihood of a person presenting with trauma related headache 6 months post-injury, but only in combination with findings indicative of clinically important injury to the cervical spine (Radanov, Sturzenegger, Di Stefano, Schnidrig, \& Aljinovic, 1993). Outcome at 12 months is primarily related to the initial severity of the injury, such as initial symptoms of radicular irritation and intensity of neck pain (Radanov, Sturzenegger, Di Stefano, \& Schnidrig, 1994). Moreover, results indicate that poor recovery is related to severity of injury in addition to some pretraumatic factors (previous history of head trauma and headache) and initial injury-related reactions (i.e. sleep disturbances, reduced speed 
of information processing and nervousness). Other variables that are predictive of a poor outcome at 12 months are impaired neck movement, history of pretraumatic headache, history of head trauma, older age, initial neck pain intensity, initial headache intensity, nerwousness, neuroticism, and impaired selective attention performance (Radanov \& Sturzenegger, 1996). Patients who were still symptomatic at 2 years post-injury were older, had a higher incidence of rotated or inclined head position at the time of impact, had a higher prevalence of pretraumatic headache, showed higher intensity of initial neck pain and headache, complained of a greater number of symptoms, had a higher incidence of symptoms of radicular deficit, and higher average scores on a multiple symptom analysis. In addition, symptomatic patients scored higher with regard to impaired well-being and performed worse on tasks of attentional functioning and showed more concern about long-term suffering and disability (Radanov, Sturzenegger, \& Di Stefano, 1995).

Cognitive deficits after CAD injury are mainly documented in the attentional domain, while "higher" cognitive functions, such as logical and conceptual thinking appear to be preserved. Attentional deficits have been demonstrated by Ettlin and collegues (1992), by Kischka, Ettlin, Heim, and Schmid (1991), by Radanov, Di Stefano, Schnidrig, Sturzenegger, and Augustiny (1993), by Radanov and collegues (1992), by Radanov, Hirlinger, Di Stefano, and Valach (1992), by Schwartz and collegues (1987), and by Yarnell and Rossie (1988), but not by Olsnes (1989). Studies on memory performance have yielded controversial results (Kischka et al, 1991; Olsnes, 1989; Yarnell, \& Rossie, 1988). Impaired cognitive function, as documented in the postconcussional syndrome (Binder, 1986), has been considered to substantially influence psychosocial adjustment after CAD (Lishman, 1973; Van Zomeren \& Van den Burg, 1985): If impairment of attention occurs following CAD, it may account for these patients" disability and problems in psychological adjustment, both of which have socioeconomic and medicolegal ramifications.

Evans $(1992)$ and Radanov and collegues $(1993,1995)$ proposed that older age is a factor that is predictive of a less favorable outcome after CAD. Houx, Vreeling, and Jolles (1991) and Jolles, Houx, Vreeling, and Verhey (1993) state that minor health-related factors (so-called Biological Life Events, BLEs) may be important in determining the age at which the physiological aging process leads to cognitive decline. Cognitive aging is characterized by a discontinuity caused by lesions in the central nervous system (Satz, 1993) which are considered to reduce the "brain reserve capacity". This threshold model is based on the assumption that brain reserve capacity is negatively affected by disease states and aging. In elderly subjects, a CAD, which according to Varney and Varney (1995) might be associated with brain injury, might make previous subthreshold symptoms clinically demonstratable. We therefore hypothesized that older symptomatic CAD patients would show a disproportional decline in attentional performance compared to healthy controls matched for age, sex, and education. A possible explanation of the inconsistency in the literature concerning neuropsychological findings might in part be explained by the fact that CAD patients may invest more effort and thus attain equivalent performance levels to those of healthy subjects. To examine this possibility, we obtained a subjective rating of the perceived effort that subjects had to invest to complete the neuropsychological testing. We furthermore examined whether CAD patients. show stronger time-on-task effects, which we considered to be indicative for a reduction in sustained information processing capacity. 
Twenty-nine young ( $<50$ years) and 20 old patients ( 250 years) suffering from $C A D$ injury were compared to 49 healthy controls who were matehed with respect to age, sex, and educational level. In the CAD group all subjects met the definition of CAD injury as proposed by Hirsch and colleagues (1988). Male and female CAD patients who were referred to a neurologist because of reduced mobility and pain of the neck were asked to participate in a complementary neuropsychological investigation. CAD patients who volunteered were subsequently screened over the telephone to ensure that none of them had a medical history of complicated TBI (i.e., bone or skull fractures), a major psychiatric illness known to be characterized by cognitive deficits, previous brain damage caused by stroke or disease, alcohol or drug abuse, impaired vision or hearing, or an insufficient command of Dutch. The time elapsed since CAD was set at minimally 6 months, in order to include only subjects with a chronic CAD syndrome (Pearce, 1994). Two patient groups were formed: the first group consisted of CAD patients younger than 50 years (group I; young), whereas the second group consisted of patients of 50 years and older (group II; old). Two control groups of 49 volunteers were drawn from a large cross-sectional study into biological and psychological determinants of cognitive aging, the Maastricht Aging Study (MAAS; Jolles, Houx, Van Boxtel, \& Ponds, 1995). Control subjects were selected by means of matching to the individual CAD subjects with respect to age ( \pm 1 years), sex, and level of education ( \pm 1 point). The latter was assessed by a Dutch scoring system (Verhage, 1964) which comprises a scale ranging from 1 ("primary education not finished") to 7 ("university degree"). There were thus two CAD groups and two control groups with individually matched pairs.

The mean age at testing of young and old CAD subjects was 35.7 $(S D=6.8)$ and 55.0 years $(S D=5.1)$, respectively; the mean age of controls was $35.9(S D=6.9)$ and 55.0 years $(S D=$ 5.5 ), respectively. In both the experimental group and the control group there were 12 male and 17 female young subjects, and 8 male and 12 female old subjects. The mean educational level of young and old controls was $4.1(S D=1.4)$ and $3.7(S D=1.3)$, respectively. In $C A D$ subjects this was 3.9 and $4.0(S D$ both $=1.7)$, respectively: These differences were not statistically significant at the 05 level.

The procedure for recruitment of the controls has been described elsewhere (Jolles et al., 1995). In short, normal, thealthy controls were drawn from a pool of 1,252 individuals who volunteered for the MAAS study in a postal survey from which medical and demographic information was also derived. Subjects for MAAS were randomlly recruited from a register of general practices (Metsemakers, Höppener, Knottnerus, Kocken, \& Limonard, 1992). Medical exclusion criteria were defined as those active or inactive medical conditions that may interfere with normal cognitive function (see chapter 5).

Subjects who volunteered for the study completed a postal questionnaire and underwent a neurocognitive evaluation which was part of an extensive neuropsychological investigation. The standardized postal questionnaire yielded a subjective report on medical history and cognitive functioning after $\mathrm{CAD}$. A checklist of postconcussional symptoms was completed, which distinguished between typical postconcussional symptoms and rather aspecific psychovegetative functional symptoms (Bohnen, Twijnstra, \& Jolles, 1992b). Subjects were asked to rate their perceived effort after completion of all the neuropsychological tests. These checklists were also 
completed by the matched controls. Group and age characteristics are summarized in Table 9.1., and show that $\mathrm{CAD}$ patients scored higher on all subjective ratings. Age or interaction effects were mot found, however.

Table 9.1

Characteristics of young and old controls and CAD patients

\begin{tabular}{|c|c|c|c|c|c|c|c|c|c|c|c|}
\hline \multirow[b]{3}{*}{ Variable } & \multicolumn{4}{|c|}{ Controls } & \multicolumn{4}{|c|}{ CAD Patients } & \multirow{3}{*}{$\begin{array}{l}\mathrm{CAD} \\
\text { Effect }\end{array}$} & \multirow{3}{*}{$\begin{array}{l}\text { Age } \\
\text { Effect }\end{array}$} & \multirow{3}{*}{$\begin{array}{l}\text { Interaction } \\
\text { Enfect }\end{array}$} \\
\hline & \multirow{2}{*}{\multicolumn{2}{|c|}{$\begin{array}{l}\text { Young } \\
M\end{array}$}} & \multicolumn{2}{|c|}{ Old } & \multicolumn{2}{|c|}{ Young } & \multicolumn{2}{|c|}{ Old } & & & \\
\hline & & & $M$ & $S D$ & $M$ & $S D$ & $M$ & $S D$ & & & \\
\hline \multicolumn{12}{|c|}{ Postconcussionall Symptomsa } \\
\hline PCS-I & 18.78 & 4.41 & 21.35 & 6.29 & 32.93 & 5.26 & 31.65 & 5.67 & $<.001$ & $n . s$. & a.s. \\
\hline PCS-II & 17.54 & 3.69 & 20.20 & 5.55 & 22.61 & 5.43 & 23.70 & 4.90 & $<.001$ & n.s. & n.s. \\
\hline Test Effort & 55.03 & 22.17 & 61.80 & 24.76 & 80.14 & 17.88 & 76.16 & 22.72 & $<.001$ & n.s. & n.s. \\
\hline
\end{tabular}

Note. Figures for Means and Standard Devations represent extensiveness of postconcussional symptoms or the magnitude of perceived test effort.

a See text for explanation

${ }^{b}$ Levels of sigmificance for CAD, Age, and CAD $x$ Age effects, n.s.: not significant

\section{$9.2 .2 \quad$ Neurocognitive Tests}

Speed of Information Processing was measured with the Motor Choice Reaction Test (Houx \& Jolles, 1993), by which RTs are studied as a function of task complexity. In the "simple" condition, the subject is requested to hold down, with the index finger, a red home button. When a single target buiton lights up, the subject has to press this button with the same finger. In the "choice" condition the subject has to press one of three possible buttons that light up.. In. the "incompatible" condition the subject has to press the button adjacent to the button that lights up. The home button has to be held down immediately after the target button was pressed until the next button lights up. The measure used for analysis is the moton initiation response time (RT) of the simple, the choice and the incompatible condition.

Stroop Color Word Test (Lezak, 1995; Stroop, 1935), is a selective attention task aiming at: measuring interference susceptibility and consists of four subtasks. The test examines the speed at which color names are read (SCWT-1) and the speed at which colored patches are named (SCWT-II). SCWT-III invalves color names again, but the printing ink is different from the color name. SCWT-IV requires more flexibility for the naming and reading of the items depending on a special visual cue given with the words. This subtask has proven to be sensitive to subtle changes in information processing capacity in mill TBY patients (Bohnen, Jolles, \& Twijnstra, 1992; Bohnen, Twijnstra, \& Jolles, 1992a). To have a measure of interference susceptibility irrespective of age, interference susceptibility is expressed by the percentage of extra time needed for SCWT-III, relative to the average of the first two stibtasks:

$$
\frac{\text { SCWTIII }-.5 *(\text { SCWTI }+ \text { SCWTII })}{.5 *(\text { SCWTI }+ \text { SCWTII })} * 100 \%
$$

The extra slowing on SCWT-IV is estimated by the subtraction of SCWT-III from SCWT-IV. 
The Concept Shifing Test (CST, Houx \& Jolles, 1994) is an adaptation of the Trail Making Test (Lezak, 1995). The CST consists of three subtasks. In each subtask, the subject has to mark small circles with digits or letrers in ascending order on an A4 sheet of paper. The subject is requested to do this as quickly as possible. On each test shet, 16 small circles are grouped in a larger circle. In the smaller circles, the test items (numbers (CSTA), letters (CSTB), or both (CSTC)) appear in a fixed random order. The subjects are requested to cross out the items in the right order in the same way as in the TMT. In three identical "zero-tasks" all 16 empty circles are to be crossed out clockwise as quickly as possible. The mean value of the three administrations are taken as a measure of basic perceptuomotor speed. The relative contribution of the need to shift between two concepts to the total time needed for subtask $\mathrm{C}$ (percentage) is computed with the formula:

$$
\frac{\operatorname{CSTC}-.5 *(\operatorname{CSTA}+\operatorname{CST} B)}{.5 *(\mathrm{CSTA}+\mathrm{CSTB})} * 100 \%
$$

where CSTA, CSTB, and CSTC denote the total RT taken to complete the test part. CSTA and CSTB merely assess the more automatic control function of selective attention, while performance on CSTC much more depends on divided attention capacity (Corrigan \& Hinkeldey, 1987; Schwartz et al., 1987).

Divided attention was measured with the Self-Paced Serial Addition Test (SPASAT), which is an adaptation of the original PASAT (see chapter 6; Gronwall, 1977). Sixty-one single-digit numbers were presented aurally in a pseudo-random order. Subject are asked to add successive digits in pairs and to give the answer aloud before the next digit is presented. For example, given the sequence $2,4,1,6$, the subject has to add $2+4$ and reply 6 , then add $4+1$ and reply 5 , then add $1+6$ and reply 7 . The sequence of digits is such that no answer exceeds 18 . As soon as the subject gives the solution, the subject is presented the next digit. If the subject forgets the last digit, two new pairs of digits are presented. This condition is registered as a "restart". The PASAT differs from our task in that the time of presentation of subsequent digits depends on the time the subjects take to give the solution. Prior to the beginning of the experiment, a demonstration with written digits is given until the subjects understand what they have to do. Subsequently, subjects participate in a training session consisting of 10 digits spoken by the experimenter until they state that they fully understand the test procedure. The measures used for analysis are the number of errors and restarts.

Sustained attention was measured with a Visual Search Task (see chapter 7). In the visual. search task, stimuli are presented in a pseudo-random order on a computer video monitor ( 640 $\times 480$ pixels resolution). Twenty stimuli are randomly assigned to a $10 \times 6$ grid, all stimuli being 2.5 centimeters apart. Stimuli are small squares of $3 \times 3$ millimeters; targets are defined as four stimuli forming a square of $2.5 \times 2.5$ centimeters. Every 1000 milliseconds two stimuli are moved to a different location in the matrix. Subjects, who sit 60 centimeters from the monitor, have to respond to targets with their dominant hand by pressing a button as quickly as possible. At the center axis the visual angle horizontally is $11.8^{\circ}$; the visual angle vertically is $7.1^{\circ}$. Thus subjects have to move their eyes to be able to detect all targets. Subjects have to respond to targets within 3000 milliseconds, otherwise the targets disappear. The total number of stimuli presented is 750 . With 45 targets, this gives an overall signal probability of 0.06 . The task lasts 
12.5 minutes. Targets are never consecutive stimuli. Three targets are presented every 50,000 milliseconds and the same number of stimuli are presented in each quadrant of the inspection area. Before the actual task is performed, there is a practice session consisting of 15 blocks in which subjects obtain feedback on how well they are doing on the task. Performance was assessed by the number of correct detections (positive responses to targets) and the number of false detections (positive responses to non-targets) of all 45 targets.

\subsection{3 : Rating Seales}

Postconcussional symptoms (Bohnen et al., 1992b). This factor analytically derived checklist of postconcussional symptoms consists of a factor concerning typical postconcussional symptoms (11 high-loading items: $\mathrm{PCS}-\mathrm{I}$ ) and a factor concerning rather aspecific psychovegetative functional symptoms (13 high-loading items: PCS-II). PCS-I and PCS-II symptoms were scored on a 4-choice Likert scale and the total scores on both scales were taken as measures for analysis.

Subjectwe effort was measured by requesting the subject to put a small cross over a 150 millimeters axis of a visual analog scale to indicate the subjective effort invested during the neuropsychological investigation (Zijlstra \& Meijman, 1989). The number of millimeters on this scalle was taken as measure of invested effort.

\subsubsection{Data Analysis}

In order to estimate global CAD, age, or CAD $\times$ Age effects two-way ANOV As were used for the following measures: RTs in the simple, choice, and incompatible condition of the Motor Choice Reaction Test; SCWT I, II, III, IV, and the interference scores derived from these measures; CSTA, CSTB, and CSTC, and the mean value of the three CST "zero-tasks"; the number of errors and restarts of the SPASAT; mean RT, mean number of correct detections and false positives of the visual search task. The accepted level of significance was set at 05 to indicate tests liable for post-hoc analysis with t-tests. The accepted level of significance for all two-way ANOVAs was set at .05 . The visual search task mean RT for 15 consecutive targets was calculated to enable the measurement of time-on-task effects. There were three clusters with 15 targets and 235 non-targets each. The first cluster of targets is referred to as block 1 , and the third cluster of targets as block 3. In order to measure time-on-task effects, performance on block I was contrasted to performance on block 3 . Overall time-on-task effects associated with CAD, aging, and time were analyzed by using repeated measures analysis of variance with group (two levels), age (two levels), and time (two levels) entered as variables. The level of significance was set at $p<.05$, to indicate which test variables were suitable for subsequent post-hoc analysis. Time-on-task effects withir subject or age groups at the beginning and at the end of the task were analyzed by using t-tests for paired samples. Additionally. Bonferroni-correction was applied. The checklist of postconcussional symptoms, and the rating scale for perceived effort were compared to neuropsychological test performance by using Pearson"s $r$. 


\section{3}

\section{Resuits}

\section{Speed of Information Processing}

Analysis of variance demonstrated a significantly slower speed af information processing in CAD patients (Figure 9.1). CAD patients were slower on the simple, the choice, and the incompatible condition $(F(1,98)=27.80, p<.001, F(1,98)=16.54, p<.001$, and $F(1$, $98)=11.10, p<.01$, respectively). An age effect or an Age $x$ Time interaction was not found. The CST "zero-tasks", reflecting perceptuomotor speed (Figure 9.2), showed definite effects of $\mathrm{CAD}(F(1,97)=22.53, p<.001)$, age $F(1,97)=4.54, p<.05$, and of Group $\times$ Age $(F(1$, $98)=27.80, p<.001$ ), indicating that old $C A D$ patients were disproportionally slower than their age matched controls. Young and ald controls did not differ on this speed measure $(t=$ $-12, d f=47, \mathrm{n.s}$.), whereas old CAD patients were significantly slower than their young counterparts $(t=-2.34, d f=43, p<.05)$.

Figure 9.1 (Ieft): Mean performance on the Motor Choice Reaction Test (vertical bars denote Standard Errors of the Mean).

Figure 9.2 (right): Mean performance on the "zero task" of the Concept Shifting Test (vertical bars denote Standard Errors of the Mean).
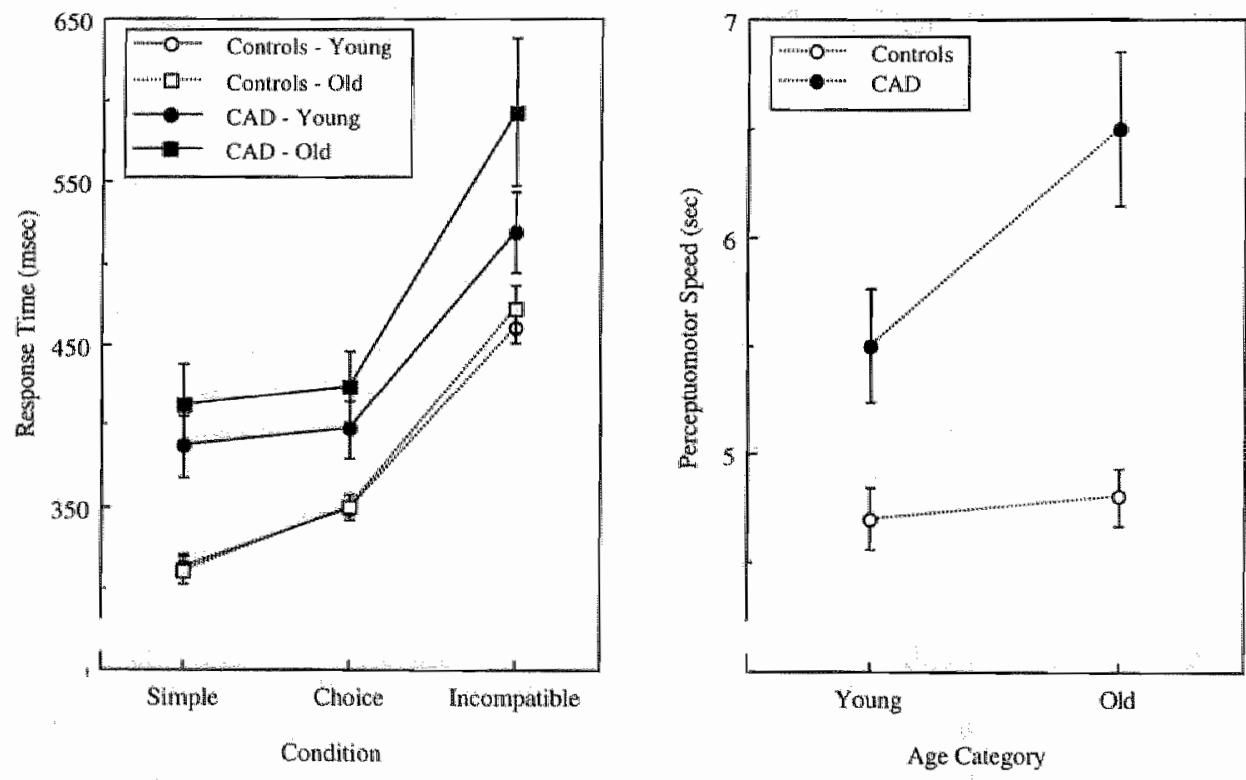

\section{Selective Attention}

CAD patients performed worse than their matched controls on all SCWT subtasks (Figure 9.3), but the differences between patients and controls became smaller when the task increased in complexity (SCWT-I: $F(1,98)=20.40, p<.001$; SCWT-II: $F(1,98)=7.83, p<.01$; SCWT-III: $F(1,98)=4.51, p<.05$, and SCWT-IV: $F(1,98)=4.68, p<.05)$. The effects of age showed the reverse pattern; no significant effects could be demonstrated on SCWT-I and 
SCWT-II (SCWT-I F $(1,98)=1.05$, n.s. SCWT-IL $F(1,98)=2.98$, n.s.), but with older age, increased task complexity had a greater effect (SCWT III: $F(1,98)=5.83, p<05$, and SCWT-IV: $F(1,98)=5.05, p<.05)$. Young CAD patients were significantly slower than young controls $(t=-2.42, d f=56, p<.05)$ and old CAD patients were significantly slower than old controls on SCWT-I $(t=-4.16, d f=38, p<.001)$. On SCWT-II, old CAD patients also performed worse than their matched controls $(t=-2.90, d f=38, p<.01)$. Age * Time effects could not be demonstrated. CAD patients did not appear to have an increased interference susceptibility, as the percentage of extra time needed for SCWT-II, relative to the average of the first two subtasks, did not differ between groups $(F(1,96)=1.39$, nis. $)$. Although not significant $(F(1,98)=3.70, p=.058)$, elderly subjects tended to be susceptible to interference, even after controlling for initial differences in speed. There were no differences between CAD patients and controls $(F(1,96)<1)$, or between young and old subjects $(F(1$, $96)<1$ ) in a test with increased interference (SCWT-RV; Bohnen et al., 1992; Bohnen at al, 1992a).

Figure 9.3 (left): Meam performance on the Stroop Collor Word Test (vertical bars denote Standard Errors of the Mean).

Figure 9.4 (right): Mean performance on the Concept Shifting Test (vertical bars denote Standard Errors of the Mean).
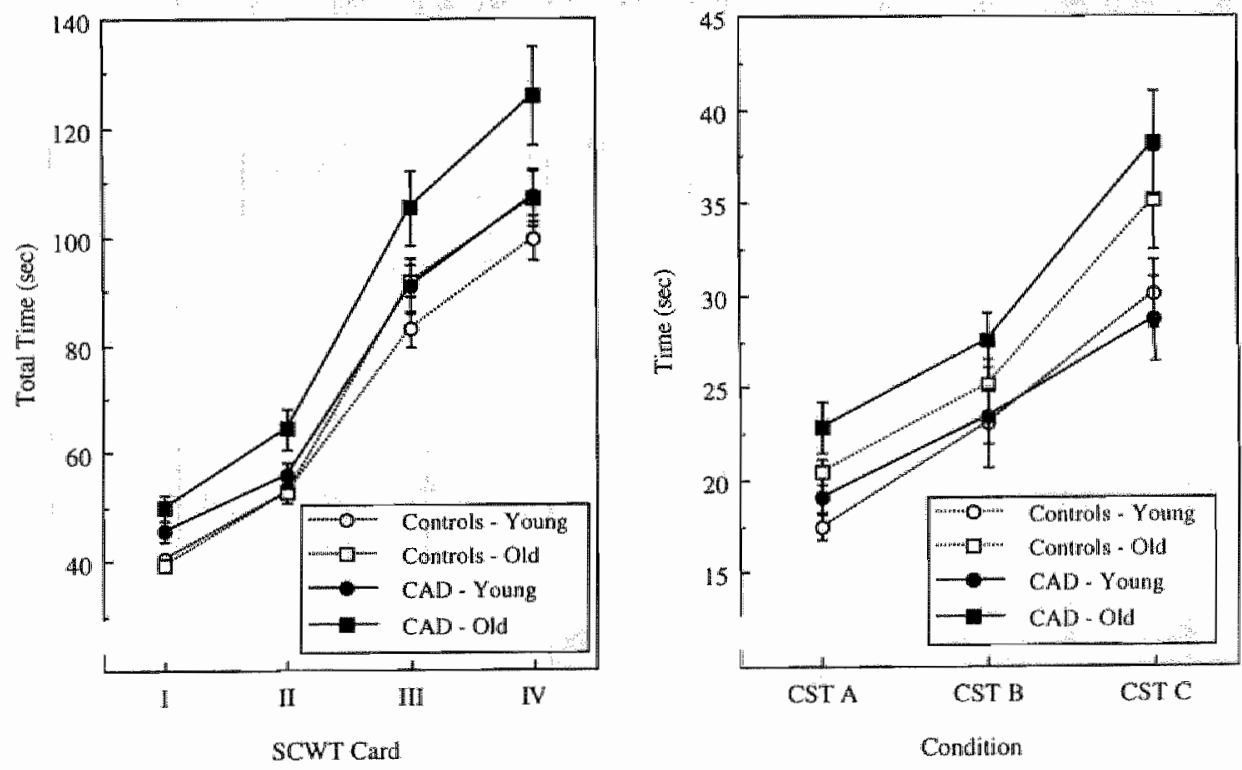

\section{Divided Attention}

The CST subtask, which assesses selective attention capacity (CSTA), showed CAD and old subjects to perform worse than controls and young subjects (Figure 9.4; Group: $F(1,98)=$ 4.32, $p<.05$; Age: $F(1,98)=11.62, p<.01)$, whereas CSTB did not show any group or age effects. In the CSTA condition, old controls were slower than young controls $(t=-2.77, d f=$ 
$47, p<.01)$ and old CAD patients were again slower than young patients $(t=-2.35, d f=47, p$ $<05$ ). The capacity to divide attention (CSTC) did not appear to be affected by the fact that a subject had sustained CAD $(F(1,96)<1)$, but was affected by age $(F(1,98)=9.21, p<.01)$. This primarily could to be explaned by the fact that old CAD patients were sllower than young patients $(t=-2.67, d f=47, p<.05)$. Performance on the SPASAT (Figure 9.5) also was not affected by $C A D$ injury (errors and restarts both $(F(1,96)<1)$. Old age, however, led to an inerease in the number of errors $(F(1,96)=1030, p<0 \mathrm{l})$, but not in the number of restarts. This effect could be attributed to the significant higher number of errors made by the old control group $(t=-3.46, d f=47, p<.01)$.

\section{Sustained Attention}

Analysis of the visual search data showed that mean RT was not affected by CAD injury or by age (Group and Age, both $F(1,98)<1$ ). The mean number of correct detections was negatively affected by CAD injury $(F(1,98)=5.13, p<.05)$, but not by age $(F(1,98)<1)$. The number of false positives did not increase as a function of CAD injury or age (Group: $F$ (1, $98)=1.79$, n.s.: Age: $F(1,98)=9.21, p<.01)$. Group $\times$ Age interactions were not found on any visual search measure.

Figure 9.5 (left): Number of emors and restarts on the Self-Paced Serial Addition task (vertical bars denote Standard Errors of the Mean).

Figuin 9.6a (right)*Response time on the Visual Search lask (vertical bars denote Standard Errors of the Mean).

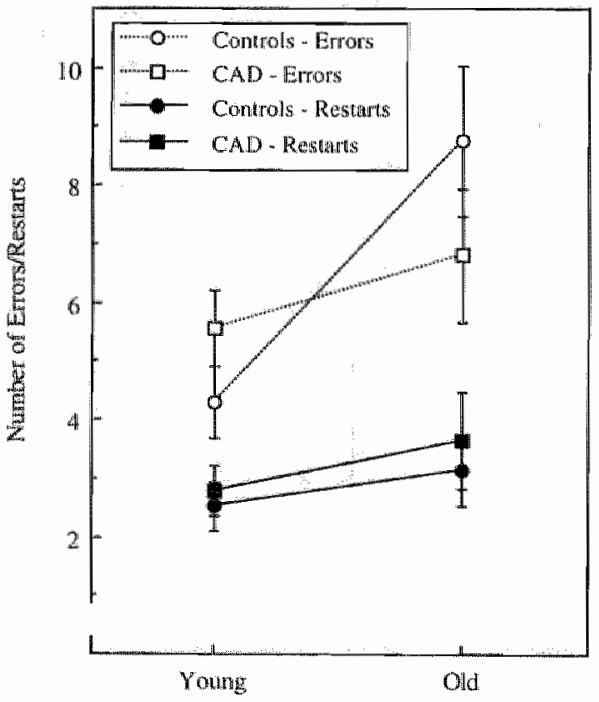

Age Category

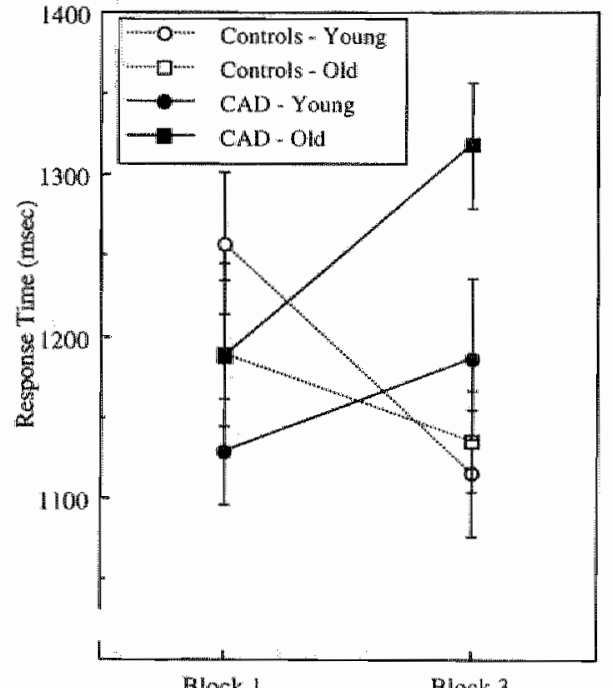

Block !

Block 3 
Figure 9.6olb (lef): Number of Correct Detections on the Wisual Search task (wertical bars denote Standard Enrons of the Mean).

Figure 9.6c (right): Number of False Positives on the Visual Search task (vertical bars denote Standard Errors of the Mean).

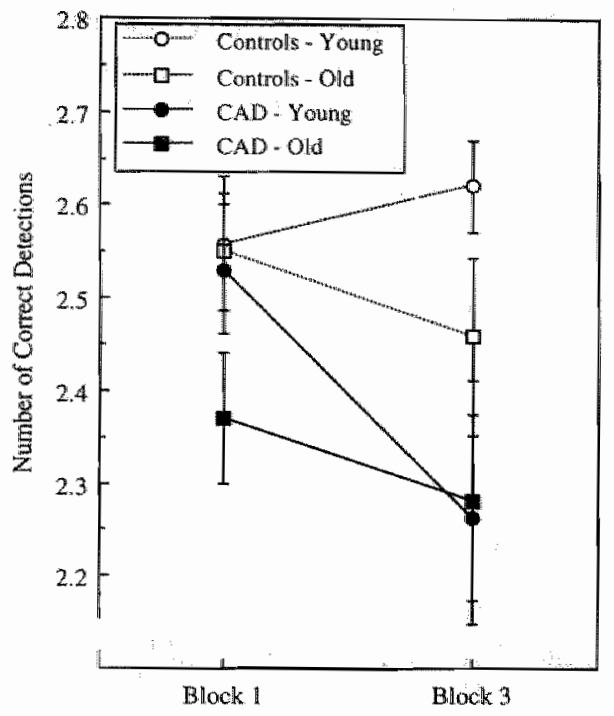

Test: Part

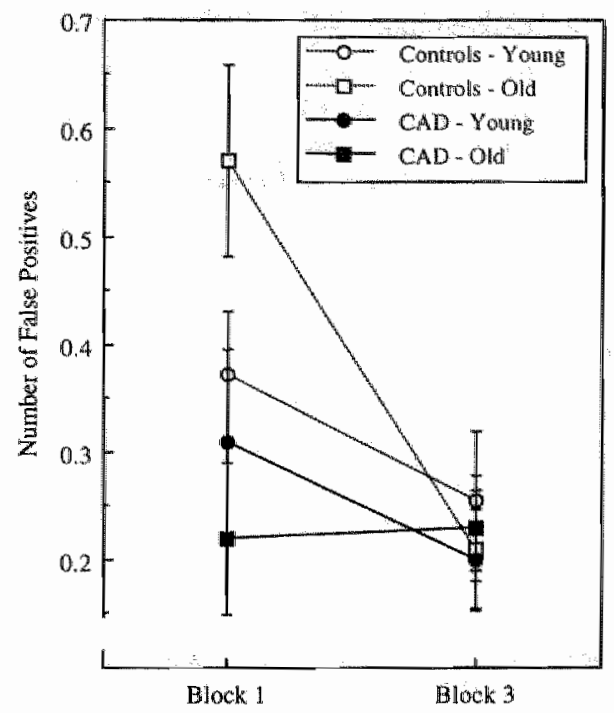

Test Part

Repeated measures analysis of variance of RT data did not show significant effects of CAD or age (Figure $9.6 \mathrm{a} ; F(1,98)<1$ and $F(1,98)=1.16$, m.s., respectively). Comparison of scores at the beginning and the end of the visual search task, however, showed a significant Group $\mathrm{x}$ Time interaction $(F(1,98)=10.87, p<.01)$. Young and old CAD injury subjects started the test faster than the young and old controls, whereas they slowed down in the last part of the test. A paired $t$-test showed that the young controls significantly gained speed from the beginning to the end of the test $(t=2.66, d f=28, p<.05)$. CAD injury affected the number of correct detections (Figure $9.6 \mathrm{~b} ; F(1,98)=8.01, p<.01)$, as did time $(F(1,98)=4.26, p<$ .05). Young CAD patients showed a significant reduction in performance towards the end of the test $(t=3.52, d f=28, p<.01$ ), whereas the performance of the other groups stayed at the same level. The number of false positives (Figure 9.6c) was also negatively affected by $C A D$ injury $(F(1,98)=4.60, p<.05)$. Analysis of data furthermore revealed an effect of time $(F(1$, $98)=10.26, p<.01)$, a Group $\times$ Time interaction $(F(1,98)=4.37, p<.05)$, and a Group $\mathrm{x}$ Age $x$ Time interaction $(F(1,98)=4.05, p<.05)$. These effects could be attributed to the fact that young CAD patients performed better towards the end of the test, i.e., they had fewer false positives $(t=3.42, d f=19, p<.001)$.

\section{Postconcussional Symptoms and Attentional Performance}

Compared to matched controls, CAD patients had more PCS-like symptoms. CAD patients had significantly more PCS-1 symptoms $(F(1,98)=129.59, p<.001)$, symptoms, which are 
considered to be cognitive energetic and traditional postconcussional complaints, and more PCS II symptoms $(F(1,98)=18.42, p<.001)$, which are considered to be rather aspecific psychovegetative symptoms well as symptoms of depression and emotional lability. CAD patients reportedly invested more effort in their overall performance $(F(1,97)=22.13, p<$ .001). The partial correlation coefficients of the relation between PCS-I and PCS-II symptoms, perceived test effort and measures of attention are summarized in Table 9.2 . It is noteworthy that PCS-II symptoms do not appear to be significantly correlated to attentional performance.

fiable 9.2

Partial Correlation Coeffieinentis Controlling for Age

\begin{tabular}{|c|c|c|c|c|c|c|}
\hline & PCS I & Sign. & PCS-I & Sign. & Tesi Effort & sign. \\
\hline Simple RT & $.280\rfloor$ & : & 1105 & - & .1930 & \\
\hline Choice RT & 3074 & $*$ & .0645 & $=$ & .2622 & $*$ \\
\hline Incompatible RT & 3186 & $*$ & .0422 & - & .2466 & $*$ \\
\hline CST" "zero task" & 1453 & $\therefore$ & .0065 & - & .2053 & \\
\hline SCWT-I & .1569 & - & -.1136 & - & .2201 & \\
\hline SCWT" «II & .0714 & - & -.0893 & - & .2681 & $*$ \\
\hline SCWT TII & .2442 & * & -.0772 & - & .2161 & \\
\hline SCWT-IV & .2121 & - & -1154 & - & .3522 & $*$ \\
\hline $\operatorname{CSTA}$ & .1257 & - & .1067 & - & .0860 & \\
\hline CStB & -.0616 & - & .0578 & - & -.0137 & \\
\hline CSTC & .1067 & - & .0955 & - & .0918 & \\
\hline SPASAT Eirors & .0741 & - & .1737 & - & 1225 & \\
\hline SPASAT Restarts & .2353 & - & .2276 & - & .3055 & 冰 \\
\hline Wisual Search R'T & .1218 & - & .1432 & - & -.0262 & \\
\hline Visual Search Correct Detections & -1166 & - & -.0313 & - & -3954 & 极济 \\
\hline Visual Search Ealse Positives & .0562 & - & .1466 & - & 1835 & \\
\hline & & & & & & \\
\hline
\end{tabular}

Note. $* 0<05$;

\subsection{Discussion}

The major question of the present study was whether young or old subjects who had sustained a CAD injury at least 6 months before are characterized by a decreased attentional performance compared to matched controls. A second question was whether there was evidence that old CAD patients showed an accelerated decline in attentional performance as a result of a diminished brain reserve capacity.

Cognitive aging is usually accompanied by a significant decrease in information processing speed (e.g., Cerella, 1990; Salthouse, 1985; for an overview see Bashore, 1993). The old CAD injury group in our study, however, did not appear to be more affected than their young counterparts. The young and old CAD patients as a group performed significantly worse than the matched controls on a speed of information processing task involving decision making (Figure 9.1). These findings are in line with the study of Stuss and coworkers (1989), who found that patients with mild TBI had a decreased information processing capacity. In addition to their findings, we moreover demonstrated that the combined effects of CAD injury and aging could lead to a disproportionate decline in perceptuomotor speed (Figure 9.2). The impact of 
CAD injury on measures of selective attention (Figure 9.3) became smaller with increasing task complexity, while the effect of age showed the reverse pattern. It is noteworthy that our CAD patients did not show an increased susceptibility to interference on the SCWT, or on a subtask that is considered to be sensitive to minor cognitive deficits. The study by Klein, Honx, and Jolles (1996) showed similar effects in patients who had sustained mild to moderate TBI several decades earlier. They also did not find an increased susceptibility to interference anong their patients, but merely found that TBI patients are characterized by a generalized reduction in the rate of information processing. In this they confirmed the results of the study by Stuss and coworkers (1985), who stated that residual brain damage secondary to TBI might be expressed primarily by an impairment in information processing capacity. Bohnen and collegues (1992, 1992a) demonstrated that patients with mild TBI who had persistent symptoms at 3 months were more susceptible to interference than patients who were asymptomatic, suggesting a relationship between recovery and cognitive performance. This relation does not appear to be particularly strong in CAD patients. Although CAD patients reported significantly more postconcussional symptoms than matched controls did, they somehow did not appear to be more susceptible to interfering information. This suggests that mild TBI and CAD injury are not identical as far as the cognitive domains are concerned, although they both appear to lead to a reduction in information processing capacity in common.

It is most striking that our CAD patients apparently did not show any deficits in divided attention performance (Figure 9.5), whereas Radanov and coworkers (1992) suggested this to be a reliable finding after CAD injury, and which Radanov and coworkers (1992) primarily attributed to headache due to cervical pathology. Di Stefano and Radanov (1995), however, who found that CAD patients who were symptomatic 2 years after the initial injury had divided attention deficits, could not demonstrate a relation between pain intensity and cognitive performance. They pointed out that these deficits may be interpreted as a change in the cognitive equilibrium of CAD patients. It is possible that symptomatic patients may encounter considerable cognitive difficulties in real-life situations requiring enhanced effort. In neuropsychological testing these deficits appear to be uncovered under time-limited conditions. When patients can adjust their processing resources to the task demands at hand, such as was the case in our self-paced version of the PASAT, patients appear to be able to reach normal levels of performance. This is of interest because earlier research has only used the paced condition, and various authors have shown impaired performance in CAD patients. Our findings with various attentional tasks and the self-paced version of the PASAT showed that although CAD patients' resources might be limited, they still are able to cope with potentially stressfull situations as long as they can match the task demands to their resources. When tasks demand externally paced speeded processing, patients" limited resources may not be sufficient and can thus lead to problems in adjustment (Van Zomeren \& Van den Burg, 1985). Therefore CAD patients may perform worse when exposed to stressfull situations in everyday life situations. If job demands, for instance, are high relative to preinjury performance, then even minor cognitive deficits can lead to ineffective skills and hence to unemployment (Adamovich, Henderson, \& Auerbach, 1985).

At first sight, sustained attention or vigilance performance did not appear to be affected after CAD injury. When time-on-task effects were considered, however, young and old CAD patients as a group appeared to start the test rather fast and showed a reduction in speed at the end of the task (Figure 9.6a). Young and old controls as a group showed a tendency to start relatively slowly and appeared to benefit from practice, which led to shorter $\mathbb{R T s}$ at the end of 
the test. This suggests that CAD patients are not able to sustain constant levels of performance over long periods of time, especially under time pressure. This observation is in accordance with their subjective ratings of invested effort. The higher levels of invested effort might furthermore in part explain the discrepancy between subjective ratings of cognitive capacity and the neuropsychological findings.

We found no correlations between aspecific psychowegetative functional (PCS-II) symptoms wid attentional performance, whereas typical postconcussional (PCS-I) symptoms tended to be weakly but significantly contelated with the speed of information processing involving decision making. We also found no robust correlations between subjective ratings of invested effort and attentional performance. This suggests that the attentional deficits seen in CAD patients might be relatively independent of the postconcussional symptoms that $\mathrm{CAD}$ patients experience after injury. Schrader and colleagues (1996) even suggested that chronic symptoms are not usually related to the car accident. Expectation of disability, a family history, and attribution of pre-existing symptoms to the trauma may be more important determinants for the evolution of the chronic CAD syndrome:

The reasons for the generalized reduced processing speed in CAD patients are still unclear, although the biological rather than the functional domain seems to be involved (neurophysiological consequences of trauma). Research on cognitive slowing in the normal population (Cerella, 1985, 1990; Salthouse, 1985) is relevant here. Hypotheses on cognitive slowing consider mechanisms such as uniform slowing of synaptic transmission (Birren, 1974) or information loss at each transmission. Another hypothesis invokes the role of the norepinephrine system (Foote \& Morrison, 1987). The norepinephrine system arises in the locus coeruleus and projects widely through the brain, in particular to the prefrontal cortex. It appears to be a critical component of cortically mediated attentional processes, serving to sharpen the difference between signal and noise. An impairment in the norepinephrine system can be hypothesized as the cause for slowing and other changes, especially in tasks involving brain areas such as prefrontal cortex with projections from the locus coeruleus (Hartley, 1992). Information that previously was processed automatically now demands controlled processing, which by defintion means that the patient has to invest more effort.

More research is needed into the cause of postconcussional symptoms, especially their neurophysiology and the factors that determine their persistence. The relationships between neurophysiological changes and behavioral and neuropsychological changes are still largely unknown. New imaging techniques, such as single photon emission tomography, and positron emission tomography, will probably play an important role in our understanding of the physiology of this disorder. Our findings stress the notion that although CAD patients might not seem to perform worse on overall measures of attention, they might prove to do so when meisures of time-on-task are considered. Future research should aim at determining the relative extent to which personal resources, such as the premorbid level of intelligence, mental. stability, and family support, can compensate for the decline in brain reserve capacity associated with aging. 


\section{$9.5 \quad$ References}

Adamowich, B. B.. Henderson, J. A., \&e Auerbach, S. (1985). Cogninve Rehabihitanion of Head Injured Patients. San Diego, CA: College Hill Press.

Aubrey, J. B., Dobbs, A. R., \& Rule, B. G. (1989). Laypersons" knowledge about the sequelae of minor head injury and whiplash. Jow mal of Newrology, Neurosurgery, and Psychiatry, $52(7), 842-846$.

Barnsley, L., Lord, S., \& Bogduk, N. (1994). Whiplash injury. Pain, 58(3), 283-307.

Bashore, T. R. (1993). Differential effects of aging on the neurocognitive functions subserving speeded nuental processing. In I. Cerella, J. Rybash, W. Hoyer, \& M. L. Commons (Eds, Adult Tnformation Processing: Limits on Loss, (pp. 37-76). San Diego, CA: Academic Press.

Binder, L. M. (1986). Persisting symptoms after mild head injury: A review of the posteoncussiwe syndrone. Joumal of Clinical and Experimental Neuropsychology, 8(4), 32:3-346.

Birren, J. (1974). Psychophysiology and the speed of response. American Psychologist, 29, 808-815.

Bohnen, N., Jolles, J., \& Twijnstra, A. (1992). Modification of the Stroop Color Word Test improves differentiation between patiemis with mild head injury and matched controls. Cinical Neuropsychologist, $6(2), 178-184$

Bahnen, N., Twijnstra, A. \& Jolles, J. (1992a). Performance in the Stroop color word test in relationship to the persistence of symptoms following mild head injury. Acta Neurologica Scandinavica, 85(2), 16-121.

Bohnem, N., Twijnstra, A., \& Jolles, J. (1992b). Post-traumatic and emotional symptoms in different subgroups of pationts with mild head injury. Brain Injwry. $6(6), 481-487$.

Brown; S. I., Fann, J. R., \& Grant, I. (1994). Pastconcussional disorder: Time to acknowledge a common source of neurobehavioral morbidity. The Jowrnal of Neuropsychiatry and Clinical Neurosciences, $6(1), 15-$ 22.

Cerella, J. (1985). Information processing rates in the elderly. Psychological Bulletim, 98(1), 67-83.

Cerella, J. (1990). Aging and information processing rate. In I. E. Birren \& K. W. Schaie (Eds.), Handbook of the Psychology of Aging, (3rd ed., pp. 201-221). New York: Academic Press.

Corrigan, J. D., Hinkeldey, N. S. (1987). Relationships between Parts A and B of the Trail Making Test. Journal of Clinical Psychology, 43,402-409.

Di Stefano, G., \& Radanov, B. P. (1995). Course of attention and menory two years after common whiplash: A two-year prospective study with age, education and gender pair-matched patients. Acta Neurologica Scandinavica, 91, 346-352.

Ettlin, T. M., Kischka, U., Reichmann, S, Radii, E. W., Heim, S., Wengen, D., \& Benson, D. F. (1992). Cerebral symptoms after whiplash injury of the neck: $A$ prospective clinical and nearopsychological study of whiplash injury. Journal of Nearology. Neurosurgery, and Psychiatry, 55(10), 943-948.

Evans, R. W. (1992). Sorne observations on whiplash injuries. Neurologic Clinics, 10(4), 975-997.

Foote, S. L., \& Morrison, J. H. (1987). Extrathalamic madulation of neocortical function. Annual Review of Neuroscience, 10,67-95.

Gronwall, D. M. (1977). Paced auditory serial-addition task: A measure of recovery from concussion. Perceptual and Motor Skills, 44(2), 367-373.

Hartley, A. A. (1992). Aitention. In F. I. M. Craik \&. T. A. Salthouse (Eds.), Handbook of Aging and Cogrition. (pp. 3-49). Hills dale, NJ: Eribaum.

Hirsch, S. A., Hirsch, P. J., Hiramoto, H., \& Weiss, A. (1988). Whiplash syndrome. Fanct or fiction? Orthopedic Clinics of North America, 19(4), 791-795.

Houx, P. J., \& Jolles, J. (1993). Age-related decline of psychomotor speed: Effects of age, brain health, sex, and education. Perceptual and Motor Skills, 76(1), 195-211.

Houx, P. J., \& Jolles, J. (1994). Wulnerability factors for age-related cognitive decline, In R. L. Isaacson \& K. F. Jensen (Eds.), The Vulnerable Brain and Environmemial Risks, (pp. 25-4i). New York: Plenum Press.

Houx, P. J., Vreeling, F. W. \& Jolles, J. (1991). Age associated cognitive dechine is related to biological life events. In K.Igbal, D.R.C.McLachlan, B.Winblad, \& H.M.Wisniewski (Eds.), Altheimer's Disedse: Brasic Mechanisms, Diagnosis and Therapeutic Stranegies, (pp. 353-358). Chichester, UK: John Wiley \& Sons.

Jolles, J., Houx, P. J., Van Boxtel, M. P. J., \& Ponds, R. W. H. M. (Eds.). (1995). Maastricht Aging Siudy: Determinants of Cognitive Aging. Maastricht. The Netherlands: Neuropsych Publishers.

Jolles, J., Houx, P. J., Vreeling, F., \& Verhey, F. R. J. (1993). Cognitive aging, biological life events and primitive reflexes. Neuroscience Research Commumications: 13(Suppl. 1), \$47-S50.

Kischka, U., Enlin, T. M., Heim, S. \& Schmid, G. (1991). Cerebral symptoms following whiplashin injury. European Newrology, 3/(3), 136-140.

Klein, M., Houx, P. J., \& Jolles, J. (1996). Long-Term Persisting Cognitive Sequelae of Traumatic Brain Injury and the Effect of Age. Journal of Nervous and Mental Disease, 184(8), 459-467.

Lezak, M. D. (1995). Neuropsychological assessmen. (3rd ed.). New York: Oxford University Press.

Lishman, W. A. (1973). Psychiatric sequelae of head trauma: A review of the postconcussive syndrome. Prychological Medicine, 3, 304-318. 
Metsemakera, J. F. M. Hoppener, P., Knotinerus, J. A. Kocken, R. J. J., \& Limonard, C. B. G. (1992) Computerized heath information in the Netherlands: A registration network of family practices. Britsh Joumal of Centeral Practice, $42(356), 102-106$.

Orsmes, B. T. (1989), Neurobehaworal findings in whiplash petients with long-lasting symptoms. Acta Nearologica Scandinatica, $80(6), 584.588$.

Ommaya, K \& Hirsh, A. E (1971) Tolerances for cerebral concussion from head mpact and whiplash in primiates. Nowmal of Bomechanic 4 (1), 1321

Pearce, I. M. (1989). Whiplash injury. A reappraisal. Jounal of Newrolagy, Newrosurgery, and Psychatry. $52(12), 1329-1331$

Pearce J. M. (1994). Polemies of chronic whiplash injury (editorial). Neurology, 44(11), 1993-1997.

Radanov, B. P., Di tefano, B. Schnidrig, A., B Ballinari, P. (1991). Role of psychosocial stress in recovery from common whiplath Lance, $338(8769), 712-715$.

laadanow, B. B. Di Stefano, G, Schnidrig, A, Sturzenogger, M., \& Alugustiny, K. F. (1993). Cogmitive functioning after conmon whiphash. A controlled follow-up study. Archives of Newrology, 50(1),87-91.

Radanow, B. P. Dworak, J, \& Valach, L, (1992). Cognitive deficits in patients after sof tissue injury of the cervical spine, Spine, $17(2), 12713$.

Radanov, B. P, Hirlinger, I, di Stefano, $G$, d $V$ alach, L. (1992). Attentiomal processing in cervical spine syndiomes. Acta Neurologiea Scandinamica, 85(5), 358-362.

Radanow, B, P, \& Stutzenegger, M. (1996). Predicting recovery from common whiplash. Europect Neurology, $36(1), 48-51$.

Reddanov, B. P, Sturtenegger, M., Di Stefano, G., \&clinidrig, A. (1994). Relationship between early somatic radological, cognitive and psychosocial findings and outcome during a one-year follow-up in 117 patients suffering from common whiplash. British Joumat of Rheumatology, 33(5), 442-428.

Radanov, B. P. Sturzenegger, M. \& Di Stefano, G. (1995). Lomg-term outcone after whiplash injury: A 2-year follow-up considering features of injury mechamism and somatic, radiologic, and psychosociall findings. Medicine, 74(5), $281-297$.

Radanoy, B. P. Sturzenegger, M., Di Stefano, G., Schnidrig, A., \& Aljinovic, M. (1993). Factors influencing recovery fiom headache after common whiplash. British Medical Joumat, 307(6905), 652-655.

Salthouse, "T. A. (1985). Speed of behaviour and its implications for cognition. In J.E. Birren \& $\mathbb{K}$. W. Schaie (Eds.), Handbook of the Psychology of Aging, (2nd ad., pp. 400 426). New York: Van Nostrand Reinhold.

Satz, P. (1993). Brain reserve capacity on symptom onset after brain injury: A formulation and review of evidence for threshold theory . Neuropsychology, 7(3), 273-295.

Schrader, $\mathrm{H}_{r i}$ Obelieniene, D., Bovim, G. Surkiene, D., Mickeviciene, D., Miseviciene, I., \& Sand, T. (1996). Natural evolution of late whiplash syndrome outside the medicolegal context. Lancet, 347(9010), 1207-1211.

Schwartz, D. P., Barth, J. T, Dane, J. R., Drenan, S. E., DeGoad, D. E., R Rowlingson, J. C. (1987). Cognitive deficits in chronic patients with and without history of head/neck injury: Development of a brief screening battery. Clinical loumal of Pain, 3,94-101

Spitzer, W. O., Skowron, M. L. , Salmi, L. R., Cassidy, J. D., Duranceau, J, Suissa, S., \& Zeiss, E. (1995). Scientific monograph of the Quebec Task Force on Whiplash-Associated Disorders: Redefining "whiplash" and its management. Sphe, $20(8$ Suppl. $18-73$ s.

Stroop, J. R: (1935). Studies of interfurence in serial verbal reactions. Joumal of Experimental Psychology" 18. $643-662$

Stuss, D. 'I, Ely, P., Hugenholta, H. Richard, M. T., LaRochelle, S., Poirier, C. A., \& Bell, T. (1985). Subtle neuropsychologicel deficits in patients with good recovery atter closed head injury. Neurosurger, $17(1), 41$ 47.

Stuss, D. T., Stethem, L. L., Hugemholtz, H., Picton, T, Pivik, J., \& Richard, M. T. (1989). Reaction time after hexd injury" Fatigue, divided and focused attention, and consistency of performance. Journal of Newrology, Newrosingery, and Psychatry, 52(6), 742-748.

Van Zomeren. A. H. \& Vata den Burg, W. (1985). Residual complaints of patients two years after sewere head injury. Joumat of Neurology, Nenrosurgery, and Psychiatry 48(1), 21-28.

Varney, N. R., d Varney, R. N. (1995). Brain injury without head injury. Some physics of automobile collisions with particular reference to brain injuries accuring without physical head trauma. Applied Newropsychology, $2,47-82$.

Verhage, F. (1964). Intelligentie en leffijd (Intelligence and Age). Unpublished doctoral dissertation, University of Groningen, "The Nethertands.

Yarnell, P. R. R Rossie, G. V. (1988). Minor whiplash head injury with major debilitation. Bnain Injury, 2(3), 255-258.

Zijlstra, F, \& Meijman, T. (1989). The meten wan mentale inspanning met behulp van een subjectieve methode (measurement of mental effort with a subjective method). In T. Meijman (Ed.), Mentale Belastimg en Werkstress (Mental Load and Work Stress), (pp. 42 61). Assen/Maastricht, The Netherlands: Van Gorcum. 


\section{Chapter 10}

\section{Concluding Remarks}

\subsection{Introduction}

As noted in the introductory chapter, the central issue of this thesis is the hypothesis that mild traumatic brain injury (TBI) may negatively affect the process of normal cognitive aging. The thesis at the same time addresses the hypothesis that the neurocognitive sequelae seen after mild TBI and with aging share common features with respect to the involvement of higher attentional functions and also have a common underlying mechanism. Both mild TBI and physiological aging supposedly compromise the reserve capacity of the brain and may thus lead to cognitive deficits; however, the neuropathological mechanisms that ultimately lead to these deficits are completely different in TBI and aging.

The experimentall studies described in this thesis were performed with the main aim to evaluate higher attentional processes in TBI and in normal cognitive aging. Much effort was put into devising clinical experiments to compare the attentional performance and related cognitive processes of young and old patients with TBI (chapter 8 and 9). A second approach was to evaluate attentional functions in healthy subjects of various ages. Again, the major issue investigated was the possible interaction between Age and TBI on neurocognitive performance. This issue has to do with the hypothesis put forward by Jolles, Houx, Vreeling, and Verhey (1993), namely that so-called Biological Life Events (BLE) can influence neurocognitive performance in healthy elderly subjects. Mild TBI, according to these authors, is a major BLE which does not receive the attention it deserves, because it may compromise brain function and. lead to a decrease in neurocognitive performance in older age, by interacting in some way with the "normal" physiological aging process. The more recent theory of Satz (1993) on brain reserve capacity is consistent with the BLE hypothesis. The present studies tried to prove this by investigating (1) whether patients with mild TBI suffered earlier in life are characterized by inferior cognitive performance in older age and (2) whether the interaction between TBI and age is characterized by simple additive effects or by an amplification effect.

\subsection{What is the Nature of the Interaction between Age and Traumatic Brain Injury?}

Comparison of the neuropsychological profiles of patients with cognitive disorders is useful not 
only for elucidating brain-behavior relationships but also for assisting clinicians making a differential diagnosis. The study of patients with mild TBT suggests that the combined effect of mild to moderate TBI and advancing age does not lead to accelerated cognitive aging, although mild to moderate TBI sustained several decades before appears to have a persistent impact on attentional performance (chapter 8). The results suggest that the effects of TBI and age are additive. This important finding suggests that TBI may shift the onset of "normal" or "usual" cognitive aging to an earlier age, thereby making the "cognitive age" of subject several years older. This notion is in line with a model of limited resources that are depleted because of the subsequent effects of CNS lesions on the brain reserve capacity subserving mental operations.

The answer to the question whether mild TBI may lead to pathological cognitive aging is "most probably not". However, if one accepts a model that supposes that traumatic CNS lesions lead to permanent loss of neural tissue, some subjects may be at higher risk. According to the brain reserve capacity model (Satz, 1993) and studies that considered predictors of outcome after TBI, patients with lower premorbid intellectual or educational levels are at higher risk for a less successful recovery. A lower intellectual level may mean that the subject is less able to compensate for cognitive deficits sustained after injury, and thus consequently might be less successful in coping with everyday life situations. Mild TBI that is sustained before physiological aging becomes apparent most likely will not increase the potential risk, although lower intellectual of educational levels combined with multiple injuries might indeed diminish brain reserve capacity. Young patients who sustain mild TBI are not expected to be severely affected, unless they have previously sustained several significant brain injuries. The brain reserve capacity is reduced, however, and in later life this might give rise to an earlier onset of cognitive decline. This hypothesis has to proven by longitudinall studies, which are currently lacking. The Maastricht Aging Study (MAAS; Jolles, Houx, Van Boxtel, \& Ponds, 1995), of which the present studies are a part, is currently performing such a longitudinal study, involving 450 healthy subjects of aged 50 and older.

The biological effects of TBI may increase the risk of pathological cognitive aging such as Alzheimer"s Disease (AD), but only through a synergistic relationship with genetic factors like apolipoprotein-epsilon 4 allele (APOE-4). Mayeux and colleagues (1995), for instance, found a tenfold increase in the risk of $\mathrm{AD}$ in community-dwelling elderly persons who had both APOE 4 and a history of TBI, compared with a twofold increase in risk in subjects with APOE-4 alone. TBI in the absence of APOE-4 did not increase risk. Apart from supporting this notion, Nicoll, Roberts, and Graham (1995) furthermore suggest that known environmental and genetic risk factors for $\mathrm{AD}$ may act additively. This genetic susceptibility to the effects of TBI may be of significance both to those who have sustained TBI previously and to those whose activities put them at risk of trauma (Nicoll, Roberts, \& Graham, 1996). Although one may regard TBI, like genetic susceptibility, to be a putative risk factor for $\mathrm{AD}$, the temporal relationship between TBI and AD warrants further investigation.

Given the fact that older age itself is the most important risk factor for AD, patients over 40 years with mild TBI should be given special attention, because they may be at higher risk for developing persisting sequelae (Evans, 1992). Mild to moderate TBI in older adults appears to produce cognitive deficits involving the same neurobehavioral areas affected in young subjects, but older people seem to be mote severely affected and take longer to recover (Goldstein et al., 1994). An explanation in terms of a reduced brain reserve capacity after mild TBI and with. increasing age is attractive, both from a theoretical as well as from a physiological point of view (Satz, 1993). Reductions in brain reserve capacity can explain threshold differences in the onset 
of elinical symptoms and in the expression of impaired test performance after acquired brain injury. This model can thus be used to develop bypotheses as to what might be the neurocognitive outcome when the interaction between TBI and age is considered.

Although attentional functioning in $\mathrm{AD}$ has not been extensively studied, avalable evidence indicates that many attentional functions are clearly impaired in early AD (Parasuraman \& Haxby, 1993). If memory impairment is considered to be an outcome of hippocampal dysfunction, the special significance of attention is that it may represent the first cognitive indicator of neocortical dysfunction in the early stages of AD. Because attentional functioning is not uniformly affected in the early stages of $\mathrm{AD}$, future research should examine patterns of impairment of specific attentional operations in mildly demented patients. This kind of research should ultimately enable the clinician to distinguish between the neurocognitive sequelae of TBI in older patients and the effects of early AD. Goldstein and coworkers (1996), for instance, showed that neuropsychological markers of memory and semantic processing may be useful in differentiating between the cognitive effects of $\mathrm{AD}$ and those of early recovery from $\mathrm{TBL}$. Such a distinction is important since the therapeutic approaches for TBI and (early) AD are very different; as is the prognosis.

\subsection{Attentional Functions and Age}

The literature reviewed in chapter 3 provides evidence that several aspects of attention decline with advancing age. Older subjects, for instance, tend to process non-relevant information much more than younger subjects, when the distinction between targets and distractors is smaller. However, there are several studies which show that the ability to seleet relevant information (i.e., selective attention) itself does not appear to be affected by age. There rather appears to be a decrease in the availability of processing resources subserving cognitive operations. When a subject has to monitor two different sources of input alternately, aging appears to affect encoding, but not the efficiency of the shifting process itself. Divided attention performance in older subjects appears to be affected by task similarity, task difficulty and practice. Divided attention tasks can be regarded as a means to increase task complexity, thus resulting in a decreased performance in the elderly. The extent to which deterioration appears to take place strongly depends on the methodology and the model used to explain age-related differences. Findings with regard to vigilance suggest that effects of age also depend on the type of task studied and the aspect of performance exarnined. Age differences in perceptual and cognitive performance are often most apparent when relatively greater demands on processing capacity are imposed on the subject. The experimental studies of this thesis on age-related attentional performance (chapters 5, 6, and 7) furthermore demonstrate that the analysis of time-on-task effects can reveal important information as to which variables predict attentional performance with advancing age. Visual and auditory acuity, for instance, explains a significant proportion of the variance in age-related attentional performance, even when deficits in acuity are corrected for by means of antificial aids. Although of great influence, minor basic perceptual deficits are usually not dealt with in most studies on cognitive aging. Future studies should take this factor into consideration. 
10.4 Simllarities and Differences between Traumatic Brain Injury and Cervical Acceleration/deceleration Injury

The study of cervical accelerationddeceleration injury (CAD) presented in chapter 9 showed only weak age-effects, probably because these young and old symptomatic patients did not differ in age as much as the young and old groups of mild TBI patients did.

Mild TBI as well as CAD injury can lead to brain damage, although there are differences in the brain regions that are usually affected after injury. TBI usually gives rise to diffuse axonal injury (Adams, Graham, Murray, \& Seott, 1982; Eisenberg \& Levin, 1989; Yokota Kurokawa, Otsikia, Kobayashi, \& Nakazawa, 1991), whereas CAD injury can affect basal frontal and upper brain stem structures (Kischika, Ettlin, Heim, \& Schmid, 1991), thus giving rise to different neuropsychological profiles. Mittl and colleagues (1994) suggest that diffuse axonal injury may represent the pathologic substrate underlying the postconcussional syndrome that occurs in a significant proportion of patients with mild TBI:

A rellable finding in mild TBI (Acimovic, Lemmon, \& Keatley, 1993; Hugenholtz, Stuss, Stethem. \& Richard, 1988, chapter 8) as well as CAD injury (Radanow, Dworak, \& Valach, 1992, chapter 9) is that these patients show deficits of information processing capacity. These deficits may not appear to be present at superficial examination, but can be provoked when time constraints or CNS stressors (hypoxia, or dual tasks) are imposed. Patients with mild TBI furthermore may show a variety of cognitive symptoms, depending on the region of impact. The cognitive sequelae after $\mathrm{CAD}$ injury are usually influenced by the injury mechanism which frequently causes pain and referred headaches of cervical origin (Croft, 1995; Radanov \& Sturzenegger, 1996). With respect to the injury mechanisms Radanov and coworkers (1992) differentiated at least two different syndromes: 1) the "cervicoencephalic syndrome", characterized by headache, fatigue, dizziness, poor concentration, disturbed accommodation, and impaired adaptation to light intensity; and 2) the "lower cervical spine syndrome", which is accompanied by cervical and cervicobrachial pain. Accident-related consequences are factors which in themselves are known to hawe a potent influence on cognitive performance. This implies that clinical neuropsychological examination in patients with $C A D$ injury should consider pain as a major factor explaining the cognitive deficits after injury, depending on the injury mechanisms.

\subsection{Mild Traumatic Brain Injury - A Useful Classification?}

As can be concluded from the previous chapters, research examining the neurobehavioral outcome of mild TBI has yielded inconsistent and contradictory findings. Such findings have been attributed to a variety of methodological weaknesses, such as faillure to consider the premorbid status of the patient, lack of control groups, and variability in outcome time points. The use of Glasgow Coma Scalle (GCS) scores of 13-15 may permit excessive heterogeneity in TBI severity and may also contribute to the variability in neurobehavioral outcome. Culotta and associates (1996), for instance, showed statistically significant differences between the frequency of abnormalities on CT scans and the need for neurosurgical intervention in patients with mild TBI with GCS scores of 13 versus 14,14 versus 15 , and 13 versus 15 . These results indicate that there are significant differences in injury severity among patients with admission GCS scores of 13 to 15 . The implicit assumption of clinicopathological homogeneity among 
patients with such scores is challenged by these data and stresses the need for more precise diagnostic criteria in the study of the neurobehavioral outcome of mild TBI. Furthermore, the physical problems of patients with mild TBI may not be difficult to assess, but the cognitive and behavioral deficits are harder to pinpoint. To evaluate these aspects, the Rancho Los Amigos Scale of Cognitive Functioning (RLA; Selzer, 1995), named after the center where it was developed, may be useful in rehabilitation settings. As patients with diffuse axonal and neuronal injury recover consciousness they go through stages. The eight stages of the RLA are: no response; generalized response; localized response; confused, agitated; confused, inappropriate, nonagitated; confused appropriate; automatic, appropriate; and purposeful, appropriate. In this sense ratings of cognitive recovery can be combined with rehabilitation training, indicating which areas of functioning demand special attention.

\subsection{Future Directions}

Although there is much about recovery from brain injury that we do not understand, we know that the process is active and dymamic and that it can achieve striking results. The mechanisms of neural degeneration following TBI are poorly understood and no adequate treatment is currently available for the prevention of traumatic brain damage in humans. The trajectory of recovery may not always be as steep as one would like, and the final level attained not as hight, but the organism is not necessarily incapable of full recovery. Of all the elements that influence recovery, confidence in recovery may be the most potent.

The fact that attentional states can now be observed physiologically and that specific brain nuclei have been identified that participate in attentional processes (e.g., Posner, 1995) suggests that new explanations of altered attentional states are in the offing. In particular, we have the promise of multilevel explanations of variations in attentional behavior in normal aging and dementia, in which factors such as arousal, intention, and motivation can be distinguished. The integration of newly developed neuroimaging methods and neuropsychological approaches will enable researchers and clinicians working in the field of cognitive aging to evaluate their theories by using physiological rather than psychological evidence. Most theories on age-related slowing are based on the assumption that there is a general slowing of all basic processes. Complex behavior -according to this view - can be explained by combining multiple single-level performance measures. It is extremely important to detect which factors affect the age-associated slowing of behavioral speed. Slowing is functionally significant to individuals, because it affects the way they perform daily tasks, such as car driving, and increases the risk of accidents.

Future research should be directed toward a better understanding of the variety of attentional processes and their hierarchical organization, how these processes can be quantified, how attention relates to everyday complex tasks, and the impact of variables such as aging, clinical illness, and lifestyle on attentional functioning. Implications of this research include improwed diagnostic tools, identification of both adverse and optimum conditions for attention and the modifiability of these conditions, and potential contributions to lifelong well-being.

Since the information that people encounter in their daily lives is increasing in density and complexity, future research should more than ever focus on the practical relevance of our current knowledge of attentional aging, and should try to apply this knowledge to reall life situations, thus providing an environment that better meets the needs of the elderly. 


\subsection{References}

Acimovic, M. L., Lemmon, J, Keatley, M. A. (1993). The importance of qualitative indicators in the assessment of mild brain injury. Lournal of Cognitive Rehabilitation, $11(6), 8-14$.

Adams, J. H, Graham, D. I. Murray, L. S, \& Scott. G. (1982). Diffuse axonal injary due to nonmissile head initury in lumans. Anmals of Neurology, 12(6), 557-653.

Croft, A. C. (1995), Biomechanics. In S. M. Foreman \& A C. Croft (Eds), Whiplash Iniuries The Cervical Acceleration Deceleration syndrone, (2nd ed, pp. 1-92). Baltimore, MD: Williams \& Wilkins.

Culotta, V. P, Sementili, M. E., Gerold, K., Warts, C. C. Wilberger; J. E., \& Marshall, L. F. (1996) Clinicopathological heterogeneity in the classification of mild head injury. Neurosurgery, $38(2), 245-250$.

Bisenberg. H. M, \& Levin, H. S. (1989). Computed tomography and magnetic resonance imaging in mild to moderate head injury. In H. S. Levin, H. M. Eisenberg, \& A. L. Benton (Eds.), Mild Head Injury, (pp. 133 141). New York: Oxford University Press.

Ewans, R. W. (1992). The postconcussion syntrome and the sequelae of mild head injury. Neurologic Clinics. $10(4), 815-847$

Goldstein, F. C., Levin, H. S., Prestey, R. M., Searcy, J., Colohan, A. R., Eisenberg, H. M., Jann, B., \& Bertolini-Kusnerik, L. (1994). Neurobehavioural consequences of closed head injury in older adults. Journal of Neurology, Newrosargery, and Psychiatry, 57(8), 961-966.

Goldstein, F. C., Levin, H. S., Roberts, V. J., Goldman, W. P., Kalechstein, A. S., Winslow, M.. \& Goldstein, S. J. (1996). Neuropsychological effects of closed head injury in older adults: A comparison with Alzhe mer's Disease, Neuropsychology, 10(2), 147-154.

Hugenholt, H., Stuss, D. T., Stethem, L. L., \& Richard, M. T. (1988). How long does it take to recover from a mild concussion? Neurosurgery, 22(5), 853-858.

Jolkes, J., Houx, P. J., Van Boxtel, M. P. J., \& Ponds, R. W. H. M. (Eds.). (1995) Maastricht Aging Sudy: Determinants of Cogative Aging. Maastricht, The Netherlands: Neuropsych Publishers.

Jolles, J., Houx, P. J., Vreeling, F. W., \& Verhey, F. R. J. (1993). Cognitive aging, biological life events and primitive reflexes. Neuroscience Research Communications. 3(suppl. 1), S47-S50.

Käschka, U., Ettlin, T. M., Heim, S., \& Sechmid, G. (1991). Cerebral symptoms following whiplash injury. European Neurology, $3 /(3), 136-140$.

Mayeux, R., Ottman, R., Maestre, G., Ngai, C., Tang, M. X., Ginsberg, H., Chun, M., Tycko, B., \& Shelanski, M. (1995). Synergistic effects of traumatic head injury and apolipoprotein-epsilon 4 in patients with Alzheimer"s disease. Neurology, 45(3), 555-557.

Mitt, R. L., Grossman, R. I., Hielille, J. F., Hurst, R. W., Kauder, D. R., Gennarelli, T. A., \& Alburger, G. W. (1994). Prevalence of MR evidence of diffuse axonal injury in patients with mild head injury and normal head CT findings. American Jownal of Neuroradiology. 15(8), 1583-1589.

Nicoll, J. A., Roberts, O. W., Gratham, D. 1. (1995). Apolipoprotein ti epsilon a allete is associated with doposition of amyloid beta-proten following head injury. Nature and Medicine, 1(2), 135-137.

Nicoli, I. A., Roberts, G. W. \& Graham, D. I. (1996). Amyloid beta-protein, APOE genotype and head injury. Annals af the New York Academy of Sciences, 777(1), 27!-275.

Parasuraman, R., \& Haxby, J. V. (1993). Attention and brain function in Alzheimer's disease: A review. Newropinchology, $7(3), 242-272$.

Posner, M. 1. (1995). Attention in cognitive neuroscience: An overwiew. In M. S. Gazzinaga (Ed.), The Cognirive Nenrosciences, (pp. 615-624). Cambridge, MA: MIT Press.

Radanov, B. P Drorak, J., \& Valach, L. (1992). Cognitive deficits in patients after soft tissue injury of the cervical spine. Spine, $17(2), 127-131$.

Radanov, B. P.\& Sturzenegger, M. (1996). Predicting recovery from common whiplash. European Neurology. $36(1), 48-51$

Satz. $P$. (1993). Brain reserve capacity on symptom onset after brain injury: A formulation and review of evidence for threshold theory. Neuropsychology, 7(3), 273-295.

Selzer, M. E. (1995). Epidemiology and pathophysiology of traumatic brain injury. Joumal of Neurological Rehabilitation, $9(2), 55-60$.

Yokota, H., Kurokawa, A., Otsuka, T., Kobayashi, S., \& Nakazawa, S. (1991). Significance of magnetic resonance imaging in acute head injury. Joumal of Trama, 3/(3), 35 1-357. 


\section{Summary}

Attention should be recognized as a prerequisite for optimal cognitive performance. When attentional performance declines with aging, we can expect that other cognitive functions will decline likewise. This thesis deals with the question whether subjects who sustained mild traumatic brain injury earlier in life, are more vulnerable to pathological forms of cognitive aging, such as is ultimately the case in Alzheimer's disease. Since both aging and traumatic brain injury appear to affect attentional performance to a considerable extent, this thesis especially focuses on this cognitive faculty as an index of possible cognitive decline. Several studies furthermore suggest that a decline in attentional performance might be one of the early markers of neuropathological aging. A possible acceleration of the usual cognitive aging is based on a model of brain reserve capacity that is affected by disease states, like traumatic brain injury, and aging. The rationale of this thesis and the questions that are addressed are discussed in chapter 1 .

Chapter 2 discusses currently used models to explain why people become slower when they age. Attention plays a major role in cognitive processing and controls the order in which cognitive operations are performed. The speed with which these operations are performed is implicitly or explicitly measured in most studies on aging and attention. In fact, speed and accuracy are the only two measures of cognitive performance that we can quantify. A discussion of slowing phenomena in the light of age-related attentional studies is relevant, since most tasks that gauge attentional performance depend on measures of speed. The generalized slowing hypothesis is one of the major notions to approximate the cognitive clanges accompariying the aging process. One of the strongest arguments in favor of a generalized slowing approach in cognitive aging research stems from the results of the so-called Brinley plot approach, by which the mean cognitive performance of old subjects is predicted from the mean performance of young individuals. Intercepts in Brinley plots are interpreted as being a reflection of peripheral or perceptual processes, whereas the slope represents central, computational processes that are sensitive to changes in task complexity. Several authors, however, have argued against the concept of general slowing, usually in the context of an argument in favor of task-specific slowing. Information processing itself does not appear to be a static set of linear steps, but rather a dynamic event, involving changes in working memory and task processes. Universal slowing of processes is mediated through higher-order components, such as working memory, and may be evidenced in performance differences by task. Different slowing factors are required for high- and low-complexity tasks. The cognitive aging hypothesis that a single slowing factor can account for interactions between age and cognitive task complexity seems to be untenable.

The distinct highly specialized brain regions that are involved in information processing do not 
age uniformly, which also does not support the idea of a generalized decline in cognitive aging. Current mainstream cognitive and developmental research is characterized by a so-called Cognitive Neuroscience Approach, which aims at integrating behavioral, psychophysiological, and neuroanatomical approaches in the study of attention.

The differences in individual trajectories of cognitive aging strongly influence the conclusions as to what extent attentional functions are affected by the process of cognitive aging. The later decades of life of most individuals are characterized by a decrease in performance in various cognitive domains. A diminished performance on tasks of memory, perceptual functions, and speed of information processing has been reported in many articles over the last decades. Aging furthermore appears to affect the ability to integrate and plan new activities, to make decisions, or to solve problems. Several authors suggest that attentional capacity may also be invariably poorer in old individuals than in young indiwiduals. Cognitive aging might not affect all attentional domains, however. This and other issues with regard to the complex relation between attention and cognitive aging are the topics discussed in chapter 3 . The primary focus is on attentional research in the perspective of cognitive aging. Studies of the effect of age on selective attention in auditory and visual tests suggest that there is some processing of whattended stimuli, with the amount of processing varying as a function of how easy it is to discriminate between targets and distractors. Older subjects tend to process non-relevant information much more than younger subjects, when the distinction between targets and distractors is smaller. The ability to select relevant information itself does not appear to be affected by age, but there rather appears to be a decrease in the availability of processing resources underlying this cognitive operation. Aging appears to affect the encoding of information, but not the efficiency of shifting attention. Task similarity appears to affect divided attention in older subjects in a negative way $\rightarrow$ as is the case in selective attention. Task difficulty and practice also appear to be important predictors of the age-related divided attention performance. Findings with regard to vigilance performance suggest that the effect of adult age on vigilance also depends on the type of task studied and the aspect of performance examined. Age differences in perceptual and cognitive performance are often most apparent when relatively greater demands on processing capacity are imposed on the subject. Thus, age differences in the rate at which vigilance decreases over time, and in perceptual sensitivity, should be more likely under the vigilance task condition in which sensitivity decreases.

The incidence and sequelae of mild traumatic brain injury are discussed in chapter 4 . Although older subjects form a minority of people affected by tratumatic brain injury, they still pose a great burden on society, since they require more care in terms of length of hospitalization and in terms of support for persisting cognitive deficits. The fact that a subject sustained head injury earlier in life might make him or her more vulnerable to neuropathological aging, such as is the case in Alzheimer's disease. The plausibillity of the hypothesis that even mild traumatic brain injury may have an influence on the usual process of cognitive aging is discussed. It is hypothesized that brain injury and aging compromise brain reserve capacity, and a model is presented that might account for both the effects of cognitive aging and the effects of head injury. When patients with mild traumatic brain injury appear to have made a full recovery, they may still show persistent impairment when exposed to metabolic (e.g., hypoxia) or cognitive stress (e.g., dual tasks) or when they reach advanced age. Longer than normal recovery times are found in patients who are older and patients who had a previous traumatic 
brain injury. The common belief is that brain damage is irreversible, and that because neural tissue is incapable of regeneration, damage to neural structures is permanent and permanently. disabling. In fact, that is only half true. Whether a subject recovers completely from midd traumatic brain injury is likely to depend on the age at which mild traumatic brain injury is sustained and the resources the subject can allocate to compensate for the cognitive functions that are compromised. Protective factors that enhance brain reserve capacity - according to the model - are a higher intellectual or educational level and in this respect are potentially important factors in predicting the outcome of the rehabilitation process after head injury. Patients who do not have these resources readily available should be offered a more supportive environinent that in part compensates for their diminished cognitive functions.

The following chapters concern experimental studies into the age-related changes in selective, divided $_{\text {s }}$ and sustained attention. The subjects who participated in the studies described in chapter 5, 6, and 7 were assigned to one of four age groups: young, 25-35 years; young middle-aged, 40-50 years; old middle-aged, 55-65 years; ald, 70-80 years:

The effect of test duration on age-related differences in Stroop interference was estimated with the Stroop Collor Word Test, which is a test of selective attention. The performance of 429 healthy subjects on this task is described in chapter 5 . The results show a clear effect of test duration on Stroop interference. The Stroop Color Word Test was run in two parts. The young group performed the first part telatively rapidly but became slower in the second test part, whereas the old group showed the reverse effect. The two middle-aged groups did not show differences between the first and second test parts. The results are interpreted in terms of a deficit in response inhibition by a controlled processing strategy. A psychological interpretation of the study findings in terms of increased cautiousness appears less probable. The first part of the test, an abridged version of the test, may prove superior to the regular version for both clinical and research purposes.

A study aimed at detecting the determinants of the age-associated decrease in divided attention on a self-paced serial addition task, the SPASAT, is described in chapter 6. The SPASAT was administered to 196 subjects. Overall age effects were found on response time, number of errors and restarts. Simple response time, arithmetic ability, primary memory capacity concept shifting ability, interference susceptibility, and degree of hearing loss contributed to performance. Speed of performance as a function of time-on-task improved in the younger groups, but not in the older groups. Repeated administration was not associated with a reduction in the number of errors in the oldest group, but the number of restarte declined in the youngest and the oldest groups, but for different reasons. No evidence was found for age-related differences in speed-accuracy trade-off mechanisms. Elderly subjects appear to be more able to monitor their own cognitive behavior, leading to a more stable performance ower time. Its psychometric characteristics and the ease of administration make the SPASAT a very suitable instrument for measuring attentional functions in large-scale studies of cognitive aging.

Chapter 7 delineates the effects of time-on-task on age differences in visual search and the extent to which age can explain differences in simple response speed, intelligence, and visual acuity. Sex furthermore was considered as a possible influential factor. A cross-sectional study involving 442 subjects showed over-all age effects on response speed and number of correct and false detections. Time-on-task effects on response speed were absent in the old group. The 
ability to detect targets or ignore distracting information, however, was susceptible to time-on-task effects, and was thus expressed only at the end of the test. This time-on-task effect suggests that there is an interaction between inhibitory processes and the availability of central processing resources in the oldest group. Simple response time, intelligence, and visual acurty significantly contributed to performance. Statistical analysis showed that changes in visual acuity with age might be an important factor influencing performance in studies of the visual domain. Results are interpreted within the Hasher-Zacks attentional framework.

Chapter 8 addresses the notion that mild to moderate traumatic brain injury may have persistent effects which become evident upon neurocognitive testing in a phase in which the effects of physiologicall aging become manifest. Neurocognitive performance was tested in 25 middle-aged and 20 old subjects who had sustained mild to moderate traumatic brain injury on average several decades earlier. The traumatic brain injury subjects regarded themselwes as normal and healthy. The neuropsychological test performance of the subjects who sustained traumatic brain injury was inferior to that of matched healthy controls on all aspects of primary and secondary memory and on the majority of tests used to measure speed of performance. There was no interaction between the effects of traumatic bran injury and those of age, and the performance of middle-aged traunatic brain injury subjects was similar to that of old controls. The results are taken to indicate that traumatic brain injury sustained earlier in life may cause permanent sequelae in specific domains of cognitive functioning and that it might attenuate the age-related decline in cognitive functioning. Most strikingly, however, these deficits were not perceived as a limiting factor in everyday life, which suggests that coping strategies may be important.

The study described in chapter 9 investigated the information processing speed and attentional capacity associated with aging of symptomatic patients who had sustained cervical acceleration/deceleration (CAD) injury. Twenty-nine young $(<50$ years) and 20 old symptomatic CAD patients ( $\geq 50$ years) were compared to 49 healthy controls who were matched with respect to age, $\operatorname{sex}_{\text {; }}$ and education. CAD patients were mainly characterized by" a reduction in information processing capacity under speeded conditions. Performance in a non-speeded self-paced divided attention task, however, was not affected by CAD injury. This suggests that the limited resources in $\mathrm{CAD}$ injury lead to a decline in performance only when time limits are imposed. Overall measures of sustained attention performance furthermore did not appear to be affected by CAD injury, but when time-on-task effects were evaluated, CAD patients showed a decrease in vigilance performance. Overall, attentional performance in older $\mathrm{CAD}$ patients was not disproportionately more affected than in younger patients. Self-reported postconcussional symptoms and invested effort might be relatively independent of the attentional deficits after CAD injury. These findings suggest that clinicians should carefully evaluate how CAD patients reach their performance. A detailed task anallysis could reveal the cognitive difficulties of CAD patients that lead to limitations in the ability to cope with everyday life situations.

\section{Conchusion}

The concluding remarks (chapter 10) state the following:

The combined effect of traumatic brain injury and advancing age does not appear to lead to 
accelerated cognitive aging, although mild to moderate traumatic brain injury sustained several decades before appears to have a persistent impact on attentional performance. The effects of traumatic brain injury and age are additive, making the subject several years older. In our study, this meant that subjects who were physically 40 years old could be classified as being 60 years old in a cognitive sense. Analysis of time-on-task performance in symptomatic CAD patients indicates that the effects of mild traumatic brain injury can only be demonstrated by using sensitive tests or by statistical procedures aiming at detecting performance decline over time. The discrepancy between neuropsychological test performance and subjective ratings of cognitive achievement suggests that intelligence and educational level may be important factors enhancing brain reserve capacity. The decreasing reserve capacity is increasingly allocated to resilience-related processes (maintenance of functioning and recovery from dysfunction) rather than growth. By means of selective optimization with compensation, old age nevertheless continues to hold the potential for selective growth. 



\section{Samenvatting}

Een goede aandachtsfunctie kan zonder overdrijving gezien worden als de belangrijkste basisvoorwaarde voor een optimale cognitieve prestatie. Als met het ouder worden de aandachtscapaciteit vermindert, kunnen we voorspellen dat andere cognttieve functies als gevolg daarvan ook achteruit zullen gaan.

Dit proefschrift gaat in op de vraag of mensen die eerder in hun leven een mild traumatisols hersenletsel (mild traumatic brain injury; TBI) hebben doorgemaakt een grotere kans lopen on bij het ouder worden slechter te gaan presteren op neurocognitieve taken. Deze wraag is van belang voor een beter begrip van de factoren die een rol spelen in versnelde c.q. pathologisehe vormen van cognitieve veroudering, warvan de ziekte van Alzheimer het meest schrinnende voorbeeld is. Aangezien met name de aandachtsfuncties zowel bij veroudering als bij TBI veelvuldig lijken te zijn aangedaan, richt dit proefschrift zich in het bijzonder op dit cognitieve domein als mogelijke indicator voor een cognitieve achterutgang. Verschillende studies suggereren bovendien dat achteruitgang in de aandachtsfuncties één van de eerste symptomen zou kunnen zijn van een pathologische vorm van cognitieve veroudering. Als theoretische basis van dit proefschrift is gekozen voor een model dat er vanuit gaat dat de reservecapaciteit wan de hersenen vermindert door ziekteprocessen, zoals TBI, en door de effecten van veroudering.

De rationale van dit proefschrift en de onderwerpen die binnen het bovengeschetste kader aan de orde komen, worden besproken in hoofdstuk 1.

In hoofdstuk 2 worden de meeste gangbare modellen besproken die gebruikt worden ter verklaring van het feit dat veroudering gekenmerkt word door een vertraging op vele cognitieve domeinen. Aandachtsprocessen zijn verantwoordelijk voor de communicatie tussen de verschillende hersengebieden die betrokken zijn bij de cognitieve verwerking en zij bepalén de volgorde waarin cognitieve operaties worden uitgevoerd. De snelheid waarmee deze operaties worden uitgevoerd worden impliciet of expliciet gebruikt in de meeste verouderings- en aandachtsstudies. De hypothese die stelt dat er bij veroudering een algemene vertraging optreedt van alle cognitieve processen is één van de meest verbreide en geaccepteerde cognitieve verouderingsmodellen. Onderzoeken waarbij de gemiddelde cognitieve prestatie van ouderen wordt voorspeld op grond van de gemiddelde cognitieve prestatie van jongeren lijkt dit idee te ondersteunen. Verschillende auteurs hebben echter gepleit woor een vertragingsmodel dat taakafhankelijk is.

Bij de verwerking van informatie lijkt er niet zozeer sprake te zijn van een vast antal stappen dat volgens een vast patroon wordt doorlopen, maar lijkt er veeleer sprake te zijn van een dynamische interactie tussen informatie die in het werkgeheugen is opgeslagen of tussen de verschillende deelprocessen die nodig zijn voor de taakuitvoering. Algemene vertriging wordt gemediëerd door hogere-orde componenten, zoals het werkgeheugen en komt tot uitdrukking in verschillende prestaties op verschillende taken. Naast het feit dat vertraging bij hoog- of laag 
complexe taken wordt beschreven door verschillende functies, bieden de verschillende verouderingstrajecten van de verschillende sterk gespecialiseerde hersengebieden die zijn betrokken bij de informatieverwerking ook geen steun aan het idee van én onderliggende vertragingsfactor.

De laatste decennia van het leven van de meeste mensen worden gekenmerkt door een achteruitgang in de prestatie in verschillende cognitieve domeinen. Een afname in geheugencapaciteit, perceptuele functies en snelheid van informatieverwerking is veelvuldig gerapponteerd. Veroudering lijkt bovendien een nadelige invloed te hebben op het plannings-en beslissingsvermogen en op het vermogen om problemen op te lossen. Verschillende bronnen suggereren dat de aandachtscapaciteit van ouderen altijd minder is dan die van jongeren. De wrag of dat geldt woor alle aandachtsdomeinen vormt - nast andere onderwerpen met betrekking tot de complexe relatie tussen aandacht en veroudering - het onderwerp van hoofdstuk 3. De primaire andacht in dit hoofdstuk is gericht op het aandachtsonderzoek dat is wertricht bimnen het veld van de cognitieve veroudering. Leeftijds-geassocieerde selectieve atandachtsstudies in het auditieve en visuele domein laten zien dat niet-geattendeerde stimuli voor een deel wèl worden werwerkt. De hoeveelheid informatie die verwerkt kan worden is sterk afhankelijk wan de mate warin de stimuli waraan aandacht moet worden gegeven (de zogenaamde "targets") en de stimuli waar niet op moet worden gelet (de zogenaamde "distmctoren") kunnen worden onderscheiden. Ouderen zullen niet-relevante informatie sneller verwerken als het verschil tussen targets en distractoren kleiner is. Het vermogen om relevante informatie te selecteren uit de omgeving lijkt bij het ouder worden echter niet te zijn aangedaan, maar er lijkt wel een afname te zijn van de energetische bronnen die kunnen worden aangesproken bij de uitvoering van de cognitieve operatie. Veroudering lijkt van invloed te zijn op het encodeer-proces, maar niet op de efficiëntie waarmee de aandacht van het ene relevante object naar het andere wordt verschoven. Als twee taken sterk op elkaar lijken heeft dat bij ouderen een madelige invloed op het vermogen om de aandacht te verdelen. Taakcomplexiteit en de ervaring met soortgelijke taakcondities lijken ook belangrijke voorspellers te zijn voor de leeftijds-geassocieerde prestatie op verdeelde andachtstaken. Vigilantie-onderzoek toont an dat de grootte van de leeftijdseffecten op dergelijke taken sterk bepaald wordt door de aard van de tak en de deelaspecten die worden onderzocht. Leeftijdsverschillen in perceptuele en cognitieve prestatie zijn het sterkst als er een groter beroep wordt gedaan op de verwerkingscapaciteit.

De incidentie on de gevolgen van mild TBI worden behandeld in hoofdstuk 4. Hoewel de groep ouderen een minderheid vormt binnen de totale populatie van mild TBI patiënten, doen zij een groot beroep op de gezondheidszorgvoorzieningen, zeker als gekeken wordt naar de duur van ziekenhuisopname en de steun die zij behoeven bij het herstelproces. Het feit dat iemand eerder in zijn leven hersenletsel heeft opgelopen, kan hem of haar gevoeliger maken voor een neuropathologische veroudering. De aannemelijkheid van de hypothese dat zelfs mild TBI van invloed zou kunnen zijn op het gebruikelijke proces van cognitieve veroudering wordt besproken in dit hoofdstuk. waarbij wordt verondersteld dat zowel TBI als veroudering een nadelige invloed kan uitoefenen op de reservecapaciteit van de hersenen. Vervolgens word een model gepresenteerd dat een verklaring geeft voor veranderingen van aandachtsfuncties na TBI en bij veroudering. Bij schijnbaar volledig herstelde patiënten met mild TBI, kunnen onder condities van metabole of cognitieve stress, of bij hogere leeftijd persisterende cognitieve stoornissen worden aungetoond. Langer dan gemiddelde herstelperioden worden vaak 
gevonden bij patiënten die ouder zijn of die eerder een TBI hebben doorgemaakt:

Aangezien beschadigd zenuwweefsel zich niet herstelt, gat men er over het agemeen van uit dat het effect van hersentetsel onomkeerbaar is en dus ook permanent invaliderend. Dit is echter gelukkig maar voor een deel waar. De vraag of een patiènt na TBI volledig herstelt, hangt af văn de leeftijd waarop het letsel is opgelopen en de "hullpbronnen" die de patient kan aunwenden om te kunnen compenseren voor de cognitieve functies die zijn aangedaan. Factoren die daarbij eveneens de reservecapaciteit van de hersenen kumen vergroten zijn - -aldus hel model- een hoger intelligentie- of opleidingsniveau, dat in die zin als belangrijke voorspeller geld voor de uitkomst van het revalidatieproces. Patiënten die niet vrijelijk over dergelijke hulpbronnen kunnen beschikken dient een meer steunende omgeving te worden geboden die woor een deel compenseert voor de bij deze patiënten afgenomen cognitieve functies.

De hierna beschreven experimenten hebben betrekking op het onderzoek aar de leeftijdsgerelateerde selectieve-, verdeelde- en volgehouden aandachtsfuncties: De deelnemers aan deze experimenten die beschreven staun in de hoofdstukken 5,6 en 7 , werden allen ingedeeld in één van vier leeftijdsgroepen: jong, 25-35 jaar; jong-middelbare leeftijd, 40-50 jaar: oud-middelbare leeftijd, 55-65 jaar; oud, 70-80 jaar.

Het testduur-effect op de leeftijdsverschillen in interferentiegevoeligheid werd geneten door de af name van een in de kliniek veelgebruikte selectieve aandachtstest, de Stroop Kleur-Woord Test. De prestatie van 429 gezonde vrijwilligers in de leeftijd van 25 tot 80 jaar op deze test stat beschreven in hoofdstuk 5. De resultaten lieten een duidelijk effect zien van testdutr op de Stroop interferentie-score, waarbij de jonge groep relatief snel begon, maar een vertraging liet zien legen het einde van de taak; bij de oude groep was sprake van het omgekeerde effect. De resultaten kumnen worden beschouwd als indicatief voor een deficiëntie in de respons-inhibitie die verloopt via een gecontroleerde verwerkingsstrategie. Een psychologische interpretatie van deze bevindingen in termen van een toegenomen voorzichtigheid bij ouderen lijkt minder aannemelijk. Een verkorte versie van de test lijkt voor klinische en grootschalige onderzoeksdoeleinden te verkiezen boven de gebruikelijke versie.

De studie die gericht is op het achterhalen van de determinanten van de leefujdsgeassocieerde achteruitgang in verdeelde aandacht op een zelf-geïnitieerde seriële optel-taak, de SPASAT (self-paced serial addition task) staat beschreven in hoofdstuk 6. De SPASAT is afgenomion bij 196 gezonde vrijwilligers in de leeftijd van 25 tot 80 jaar, ingedeeld in de eerder getioemde vier leetijdsgroepen. Overall leeftijdseffecten werden gevonden op de reactietije (RT), het aantal fouten en het aantal herstarts. Enkelwoudige RT, rekenvaardigheid, primaire geheugen capaciteit, de concept-shifting capaciteit, de interferentiegevoeligheid en de mate van gehoorswerlies waren wan invloed op de testprestatie. Taksmelheid als aen functie van time-on-task verbeterde in de eerste twee groepen, maar niet in de oudere groepen. Herhaalde afname van de taak leidde niet tot een foutenreductie bij de oudste deememers, maar het aantal herstarts nam bij zowel de jongste als de oudste groep af, zij het om verschillende redenen. Er werden geen aanwijzingen gevonden voor leefijds -geassocieerde verschillen in speed-accuracy trade-off mechanismen. Opmerkelijk is dat ouderen in vergelijking tot jongeren beter in staat leken te zijn om hun eigen cognitieve gedrag te controleren, hetgeen in deze studie leidde tot een meer stabiele prestatie over de tijd heen.

Hoofdstuk 7 schetst de time-on-task effecten op de leeftijds verschillen in een visuele zoektak 
en de mate warth leeftijdseffecten kunnen worden toegeschreven aan leeftijds-gerelateerde verschillen in enkelvoudige RT, intelligentie, geslacht, en visus-scherpte. Een cross-sectionele studie onder 442 gezonde vrijwilligers in de leeftijd van 25 tot 80 jaar (4 groepen) toonde overall leeftijdseffecten op de RT en hel antal correcte en foute warnemingen. Time-on-task effecten op de RT werden niet gevonden in de oudste groep. Het wernogen om targets te detecteren of om distractors te negeren werd wèl beinvloed door time-on-task effecten en kwam tot uiting tan het einde van de taak. Dit effect suggereert een interactie tussen inhibitoire processen en de beschikbarheid van centrale "verwerkingsbronnen" in de oudste groep. Enkelvoudige RT, intelligentie en visus-scherpte hadden een significante invloed op de testprestatie. Statisische analyse liet zien dat leeftijdsgeassocieerde veranderingen in visusscherpte invloed hebben op de prestatie in cognitieve veroudering studies die betrekking hebben op het visuele domein.

Hoofdstuk 8 betreft het onderzoek naar de hypothese dat mild tot matig TBI blijvende effecten heeft die bij neuropsychologisch onderzoek tol uiting komen op het moment dat de effecten van fysiologische veroudering manifest worden. De neuropsychologische prestatie van 25 vijiwilligers van middelbare leeftijd en 20 oude vrijwilligers, die gemiddeld enkele decennia geleden mild tot matig TBI hadden opgelopen, werd onderzocht. De deelnemers beschouwden zichzelf als normaal en gezond. De neuropsychologische testprestatie van de hersenbeschadigden was ünferieur aan die van gematchde gezonde controle-personen op alle maten voor primair en secondair geheugen en op de meerderheid van taken die snelheid van taakprestatie meten. Er werd geen interactie gevonden tussen de effecten van hersenletsel en leeftijd. De prestatie van personen van middelbare leeftijd met TBI en die van oude controlepersonen was vergelijkbaar. De resultaten suggereren dat mild tot matig hersenletsel dat eerder in het leven is opgelopen blijvende effecten kan hebben op specifieke cognitieve domeinen. Het meest opmerkelijke in deze studie is dat deze cognitieve defecten niet als beperkend werden ervaren in het dagelijks functioneren, suggererend dat coping-strategieën grote invloed kunnen uitoefenen op het herstelproces na TBI.

De studie die in hoofdstuk 9 staat beschreven betreft het onderzoek naar de leeftijds-effecten op de snelheid van informatieverwerking en aandachtscapaciteit bij symptomatische patienten met een cervicaal acceleratie/deceleratie letsel (CAD). Negenentwintig jonge en 20 oude symptomatische Patienten met CAD letsel werden vergeleken met 49 gezonde gematchde controle-personen. Patiënten met CAD-letsel werden voomamelijk gekenmerkt door een afname in de informatieverwerkings-capaciteit onder tijds-gelimiteerde condities. De verdeelde aandachtsprestatie op een zelf-geinitieerde seriele optel-taak, de SPASAT, liet geen verschillen zien mett controle-personen. Dit suggereert dat de beperkte verwerkingscapaciteit bij patiènten met CAD-letsel alleen dan tot een prestatievermindering lijkt te leiden als een tijdlimiet wordt opgelegd. Volgehoudem aandachtsfuncties leken aanvankelijk niet te zijn aangedaan, maar bij de analyse van time-on-task effecten lieten patiènten met CAD-letsel een afname zien in hun vigilantie-niveau. Over het algemeen lijken de aandachtsfuncties bij oudere patiënten met CADletsel niet meer te zijn aangedaan dan bij jongere patiënten. Zelf-gerapporteerde postcommotionele symptomen en de geinvesteerde effort lijken relatief onafhankelijk te zijn van de andachtsstoonissen die optreden na CAD-letsel. Deze bevindingen maken duidelijk dat clinici zeer nauwgezet dienen na te gaan hoe CAD patiënten komen tot hun uiteindelijke prestatie. Een gedetailleerde taak-analyse kan de cognitieve dysfuncties van patiènten met CAD- 
letsel aantonen die leiden tot beperkingen in de mogelijkheid om tegemoet te komen aan de eisen van alledaagse situaties.

\section{Conclusie}

Het gecombineerde effect van TBI en veroudering lijkt niet te leiden tot een versnelde cognitieve veroudering, hoewel mild tot matig hersenletsel dat enkele decennia eerder is opgelopen wel blijvende effecten op de aandachtsfuncties lijkt te hebben. Leeftijd en traumatisch hersenletsel lijken eerder additieve effecten te hebben, waarbij de getroffene in cognitief opzicht een aantal jaren ouder wordt. In onze studie betekende dat dat personen die fysiek een leeftijd hadden van 40 jaar, in cognitief opzicht konden worden geclassificeerd als zijnde 60 jaar. Analyse wan de time-on-task prestatie van patiënten met CAD-letsel toont aan dat de effecten van mild TBI soms alleen tot uiting komen door het gebruik van gevoelige testen of door statistische procedures die de prestatie over de tijd heen kunnen analyseren. De discrepantie tussen de neuropsychologische testprestatie en subjectieve inschatting van de cognitieve prestatie suggereert dat intelligentie en opleidingsniveau belangrijke factoren kunnen zijn die de reserwecapaciteit van de hersenen kunnen vergroten. De bij weroudering nog resterende en verder afnemende reservecapaciteit wordt in toenemende mate aangewend voor de handhaving van functies en woor het herstel na beschadiging in plats van voor groei. Door de selectieve optimalisatie van mechanismen die voor de achteruitgang moeten compenseren is er bij veroudering toch nog potentieel plaats voor een selectieve groei. 
$\therefore$ 


\section{Dankwoord}

Sinds de inhoudelijke afronding van dit proefschrift zijn inmiddels al weer vele maanden verstreken. Maanden die gevuld werden met gedachten over de planning van mijn nieawe onderzoek bij patiènten met hersentumoren, zorgen over de woningruil die maar niet wil vlotten, de vrees dat die ene typefout pas ontdekt wordt als het proefschrift in zoveel honderdvoud is gedrukt. Zo vlak voor de gang naar de drukker wil ik een auntal mensen bedanken. Niet omdat dat gebruikelijk is, maar omdat dat terecht is.

In de eerste plaats wil ik Anouk bedanken, die op het moment dat zij in Polen zat en ik in Amsterdam, vanuit een koude telefooncel in Warschau benadrukte dat ik moest doen waar mijn hart lag. Omdat die bij het onderzoek lag, betekende dit voor mij dat ik naar Maastricht zou verhuizen, naar de groep van Jolles. Jelle Jolles als promotor en Peter Houx als dagelijks begeleider.

Beste Jelle, ik wil je danken voor al het werk dat je als mijn promotor hebt gedaan. Bij aanvang van mijn onderzoek heb je me geholpen bij het bepalen van mijn onderzoeksonderwerp. lk ben je dankbaar dat dat uiteindelijk hersenletsel is geworden. Het bleek voor mij een onderzoeksgebied waar $\mathrm{ik}$ in ruime mate en met veel genoegen de relatie tussen cognitieve functies en "het substraat" heb kunnen bestuderen. Tevens ben ik je erkentelijk voor het feit dat je bij de uitwoering van het onderzoek af en toe de teugels hebt aangetrokken, waardoor het onderzoek niet alle kanten opging als gevolg van mijn enthousiasme. Naast jouw inhoudelijke bijdragen heb je me leren inzien dat onderzoek doen ook afhankelijk is van factoren alls personele inzet, contacten, financiêle middelen en tijd, aspecten die me bij mijn nieuwe baan goed vain pas komen.

Beste Peter Houx. Mijn eerste associatie bij onze kennismaking was er een die lag tussen Gerrit Komrij en Godfried Bomans. Later ben ik je meer gaan associêren met Arendsoog, omdat elke grammaticale of typografische oneffenheid op het beeldscherm door jou terstond werd opgemerkt. Achteloos kon je binnen lopen en dan - wijzend op bet beeldscherm- melden: "Dat schrijf je met een z". Vooral ben ik je gaan waarderen door je noodzakelijk relativerende kijk op het onderzoek en je bereidwilligheid mij "te allen tijde" bij te staan in zowel vakmatige als persoonlijk associatieve aangelegenheden.

Beste Jeanette Dijkstra, jou wil ik in het bijzonder bedanken. Samen met een aantal anderen beginnen in een nieuwe omgeving is een goede ervaring. Nog prettiger is het als die anderen personen blijken te zijn waarvan je kan zeggen dat ze meer zijn dan een collega waar je af een toe een borrel mee drinkt. Jij was er eén van. Een ander die mijn verblijf in Maastricht heeft veraangenaand is Rudolf Ponds, die zo ongelooflijk hard kon schieten dat ik blij was dat ik als matig keeper in zijn team zat en niet bij de tegenpartij. Dit heeft echter niet kunnen verhinderen dat ik door toedoen van één van de Rudolf Pondsen van de tegenpartij inmiddels 
ervaringsdeskundige ben geworden op het gebied van hersenletsel. Wim Riedel wil ik bedanken voor de kritische methodologische discussies over "aspecten van de neuropsychologie". Martin van Boxtel, in de tijd dat ik in Maastricht zat was jij die andere Martin. Ik was onder de indruk van je kennis van statistick en obscure muziekjes uit de 60'er en 70'er jaren. Eveline van de Velde, jou wil ik bedanken woor je onderzoek bij een deel van de patiëntenpopulatie. Nico Rozendaal, bedankt yoor die handige programma's en door jou voorbewerkte databestanden. Astrid Quist, bedankt voor het organisatiewerk van de MAAS-studie.

Bij het lezen van het dankwoord zou het kunnen lijken dat het niet noemen van iemands naam betekent dat deze niet bepalend is geweest voor de periode die ik in Maastricht heb vertoefd. Niets is echter minder waar. Mede door de collega's die mij stimuleerden als het even niet zo vlotte met "de wetenschap" kan ik terugkijken op een goede tijd in Maastricht.

Lieve ouders, jullie will ik bedanken voor de vrijheid en het respect die ik van jullie heb gekregen, ook al waren mijn plannen misschien niet altijd even goed te doorgronden. 


\section{Curriculum Vitae}

Martin Klein is geboren op 9 februari 1961 in Amersfoort. Na de HAVO en een HBO-opleiding Maatschappelijk Werk (specialisatie Psychiatrie en bijvak Bestuurlijk Welzijnswerk) startte hij in 1984 met zijn studie Psychologie aan de Universiteit van Amsterdam. In 1989 studeerde hij "Met genoegen" af in de Psychofysiologie met de specialisatie Experimentele Psychologie. Als bijvakken volgde hij klinische Neurologie en Sociaal-Wetenschappelijke Informatica. Tijdens zijn studie deed hij onderzoek op de afdeling Klinische Neurofysiologie van het Academisch Medisch Centrum Amsterdam en liep hij een onderzoeksstage op de psychogeriatrische screeningsafdeling van het Provinciaal Ziekenhuis te Santpoort. Na een verblijf van een half jaar in Azië, werkte hij bij het Fonds voor de Nederlandse Film waar hij betrokken was bij de beoordeling van subsidieaanvragen voor korte en lange speelfims. Voor de periode van een jaar was hij als projectcoördinator voor postdoctoraal informatica-onderwijs aangesteld bij de Faculteit der Wiskunde en Informatica van de Universiteit van Amsterdam. In 1990 en 1991 verrichtte hij als chef de clinique van de afdeling Klinische en Neuropsychologie van het Onze Lieve Wrouwe Gasthuis te Amsterdam op consultbasis neuropsychologische diagnostiek bij klinische en poliklinische patiënten. Het onderzoek dat in dit proefschrift beschreven staat is van 1992 tot 1996 verricht bij de vakgroep Psychiatrie \& Neuropsychologie van de Faculteit der Geneeskunde van de Universiteit van Maastricht. Vanaf augustus 1996 is hij werkzaam bij de vakgroep Medische Psychologie van de Faculteit Geneeskunde van de Vrije Universiteit te Amsterdam. In samenwerking met verschillende specialismen en ziekenhuizen verricht hij onderzoek naar de invloed van hersentumoren en de behandeling hiervan op de cognitieve functies en kwaliteit van leven van patiënten met een hoog- of laaggradig glioom. 